\title{
Equivalence Ratio Gradient Effects on Local Flame Front Topology and Heat Release Rate in Stratified, Iso-Octane/Air Turbulent V-Flames
}

\author{
Patrizio Christian Vena \\ A thesis submitted to the Faculty of Graduate and Postdoctoral Affairs in partial \\ fulfillment of the requirements for the degree of \\ Doctor of Philosophy \\ in \\ Mechanical Engineering \\ Carleton University \\ Ottawa, Canada \\ (C)2014 \\ Patrizio Christian Vena
}


"In the middle of difficulty lies opportunity."

Albert Einstein 


\section{Abstract}

This thesis details the development and application of an experimental apparatus and methodology capable of quantifying the effects of partial premixing on the local behaviour of turbulent flames. A novel rectangular slot burner was used to generate controlled, transverse variations in mixture strength along its exit plane, such that turbulent, rod-stabilized V-flames could be anchored in reactant mixtures of varying mean gradients in equivalence ratio. A unique windowing approach was devised in which 3-pentanone tracer planar laser induced fluorescence (PLIF) of the reactants was used to determine an analysis region of interest (ROI) within the flame. Iso-contours of equivalence ratio (e.g. $\phi=0.95-1.05$ for nearstoichiometric flame regions) were traced up to the mean position of the flame front to define the width of the ROI, which was specific to each mean $\phi$ gradient flame condition. This analysis methodology enabled fair comparison among gradient settings, and ensured that any observed variation in local flame behaviour could be attributed specifically to mean $\phi$ gradient effects, rather than simple mixture strength effects. Instantaneous flame realizations were obtained from near-simultaneously acquired $\mathrm{OH}$ and $\mathrm{CH}_{2} \mathrm{O}$ PLIF image pairs. The reaction zone of the flame was identified from heat release rate (HRR) images that were calculated from the pixel-by-pixel product of the spatially registered $\mathrm{OH}$ and $\mathrm{CH}_{2} \mathrm{O}$ fluorescence signals. Resulting flame data were analyzed within the previously determined ROI for specific mean $\phi$ gradient effects on the local flame topology and HRR.

This methodology was subsequently applied to locally rich, near-stoichiometric, and lean flame regions in stratified, iso-octane/air turbulent V-flames, resulting in several important insights relevant to the fundamental understanding of partially premixed flame behaviour. First, results demonstrated that mean $\phi$ gradients had little influence on local flame surface density (FSD) and curvature probability density functions (PDFs) in near stoichiometric flame regions when comparing among consistent ranges of mean $\phi$ within the ROI. Variations were less significant than those reported in the literature, and suggested that 
mean gradient effects may be less important in near-stoichiometric flame regions at the current turbulence levels.

Second, subtle differences in HRR and instantaneous flame thickness were observed among reference-premixed and gradient flame conditions for locally rich, near-stoichiometric, and lean flame regions. Results implied that back-support from the heated products altered the HRR (and hence the local reaction zone) along the stratified branches of the V-flame, and further suggested that different mechanisms of back-support may occur between locally rich and lean flame regions.

While the effects of mean $\phi$ gradients on the local behaviour of flames were generally small, it is important to note that variations were observed for flame regions in which the mean local stoichiometry was held constant, and were separate from potential effects of differences in local mixture strength. Results consistently provided evidence of the potential interaction between neighbouring flame regions, despite being limited to relatively weak spatial gradients in equivalence ratio. Conversely, the much steeper $\phi$ gradients observed in stratified charge engines and gas turbines are expected to influence flame behaviour much more significantly.

Third, global stratification effects in mixtures with different ranges in equivalence ratio were much stronger than those of mean $\phi$ gradients. Results highlighted the competing effects of flame surface generation and HRR/flame length on the total heat release rate of a flame system, and further stressed the importance of clearly defining the question and measurement approach when attempting to discern specific effects of partial premixing on combustion. The influence of equivalence ratio gradient on local flame properties may differ significantly from the influence of general stratification on the global flame system in the form of different ranges of mixture strengths; distinguishing between these effects is critical to the proper interpretation of data, where both may be relevant in different applications. 


\section{Acknowledgements}

I would like to express my special appreciation to my supervisor, Professor Dr. Johnson. His passion for research has been an inspiration over the years, and his forensic scrutiny when designing experiments and analyzing data have shaped my approach to research. Dr. Johnson, your unwavering patience, support, and dedication have been instrumental to my academic development. You've been an exemplary mentor and friend, and for that I owe you a great debt of gratitude. Thank you for sticking by me over the years, it's been a pleasure working with you, and I've learned a great deal. As you so eloquently put it, "it's been a hell of a ride". Now let's open that Brunello!

I would like to acknowledge the staff at the National Research Council. Thank you for providing access to lab facilities and equipment, for your help in setting up experiments and conducting simulations, and for conveying your technical expertise through insightful discussions. I very much enjoyed my time at M-9; your contributions were essential to the success of the project.

À Béatrice, ma partenaire de labo, merci pour tous les moments de manips que nous avons partagés ensemble. Merci pour tout ce que tu m'as appris, pour ton enthousiasme scientifique, et pour ta bonne humeur que tu distribues autour de toi.

To all my friends, starting from l'École Internationale de l'Outaouais, the University of Ottawa, Carleton University, most recently at l'Institut National des Sciences Appliquées de Lyon, and all those in between, you've helped more than you know. Thank you for reminding me that life does in fact exist outside the lab. I'm lucky to have shared so many great moments with you, and I look forward to the next.

Catherine, les mots ne suffisent pas pour exprimer ma profonde gratitude à ton égard. Merci de n’avoir jamais cessé de croire en moi, je t'aimerai toujours.

Mom, Dad, Al, I admire your generosity, devotion, and strength. Your calming influence and eternal optimism have helped guide me through times good and bad. Knowing you're by my side has given 
me the courage to pursue my goals. I take you everywhere I go, and I would not be here without your unconditional love and support. Thank you for everything. We did it. 


\section{Table of Contents}

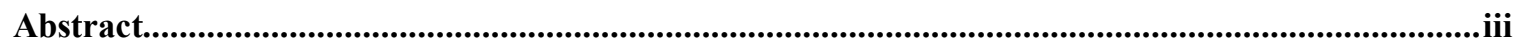

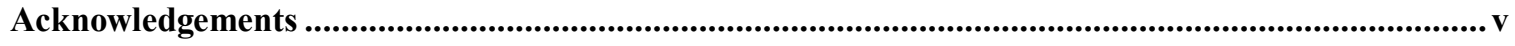

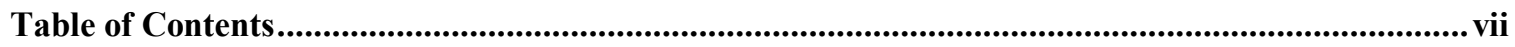

List of Tables.................................................................................................................................................... $\mathrm{x}$

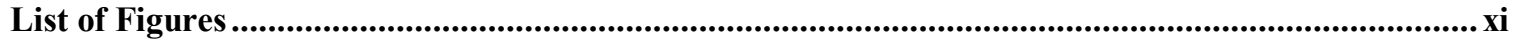

Nomenclature....................................................................................................................................................... xiii

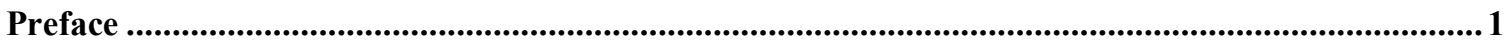

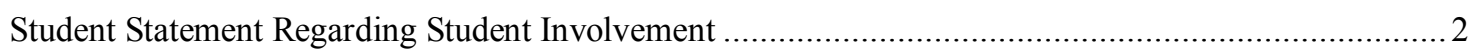

Supervisor Statement Regarding Student Involvement................................................................... 2

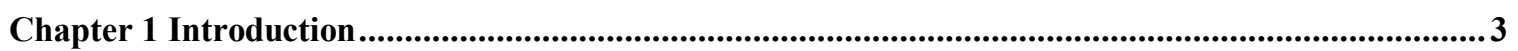

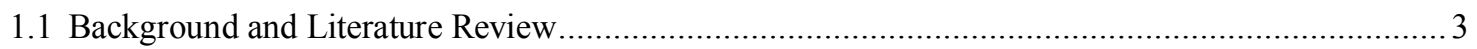

1.1.1 Variations in Local Flame Speed and Flammability Limit .................................................. 5

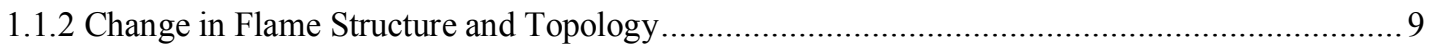

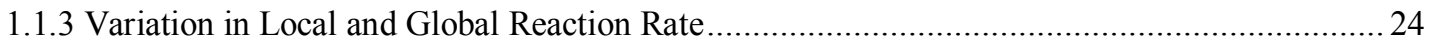

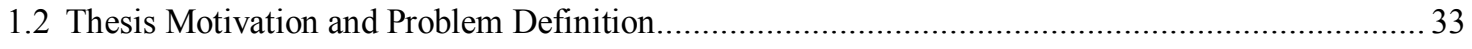

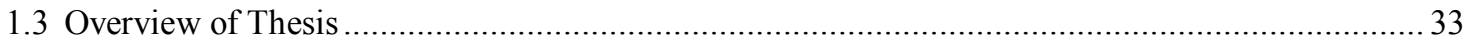

Chapter 2 Equivalence Ratio Gradient Effects on Flame Front Topology in a Stratified IsoOctane/Air Turbulent V-Flame.................................................................................................................... 37

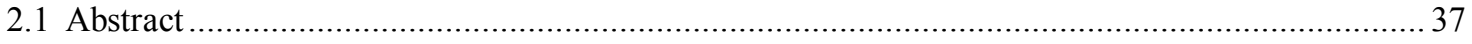

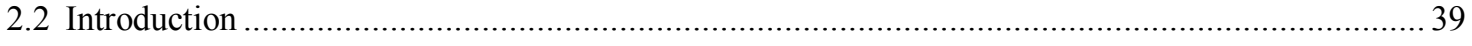

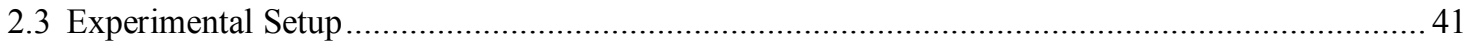

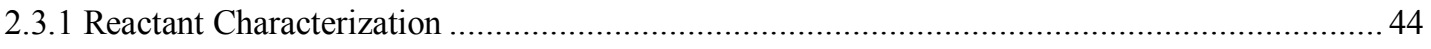

2.3.2 Determination of the Location of the Stoichiometric Surface …............................................... 45

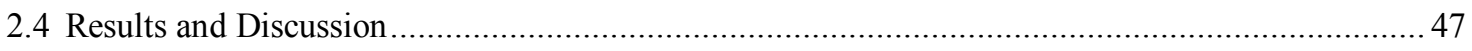

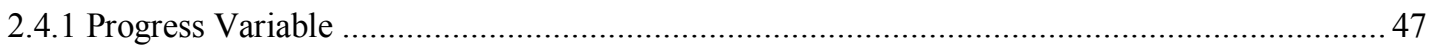

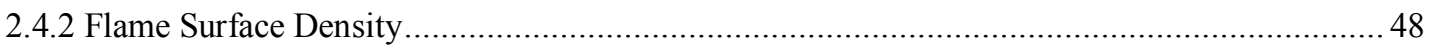

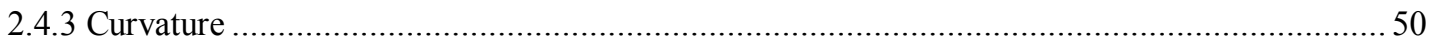

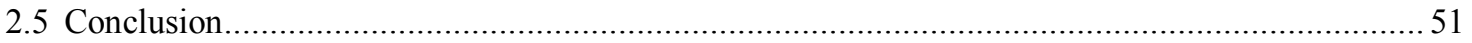

Chapter 3 Heat Release Rate Variations in a Globally Stoichiometric, Stratified Iso-Octane/Air Turbulent V-Flame..................................................................................................................................5 


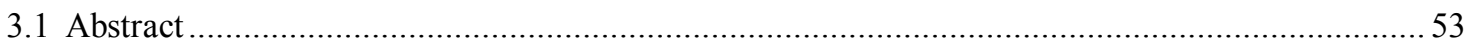

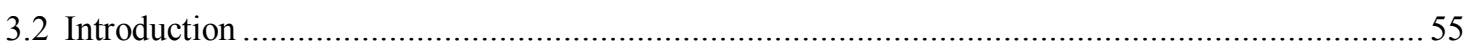

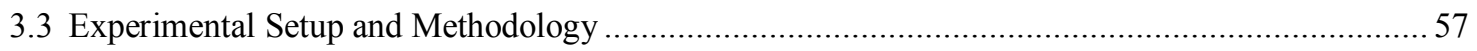

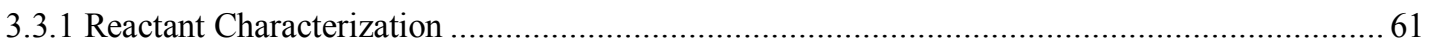

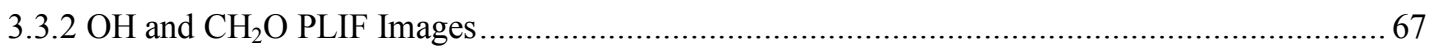

3.3.3 Determination of the Location of the Flame Front and Turbulent Flame Brush Thickness ....... 73

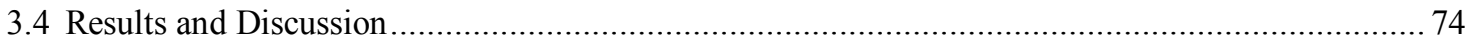

3.4.1 Instantaneous $\mathrm{OH}, \mathrm{CH}_{2} \mathrm{O}$, and Heat Release Rate PLIF Images ............................................ 74

3.4.2 Equivalence Ratio Gradient Effects on Heat Release Rate .................................................... 79

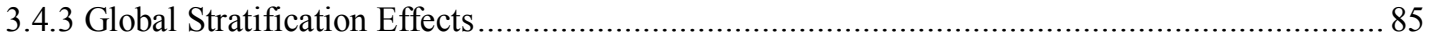

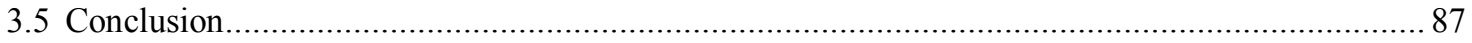

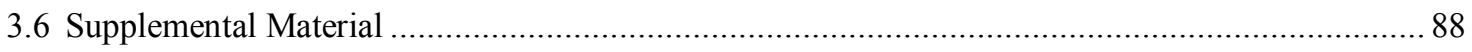

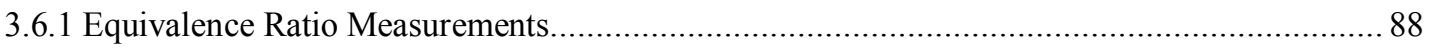

3.6.2 Correlation between (OH LIF) $\left(\mathrm{CH}_{2} \mathrm{O}\right.$ LIF) and $\mathrm{k}(\mathrm{T})[\mathrm{OH}]\left[\mathrm{CH}_{2} \mathrm{O}\right]$ Signals............................ 89

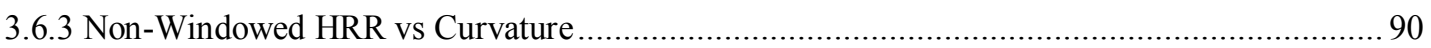

3.6.4 Physical Representation of the Effects of Mean $\phi$ Gradients on the Local HRR of Near-

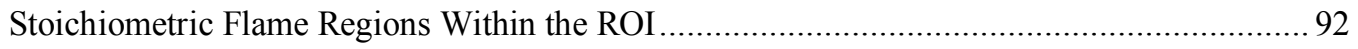

\section{Chapter 4 A Comparative Analysis on the Effects of Stratification on Locally Lean, Near-} Stoichiometric, and Rich Iso-Octane/Air Turbulent V-Flames ......................................................... 93

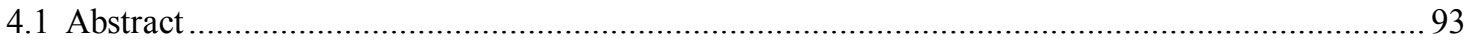

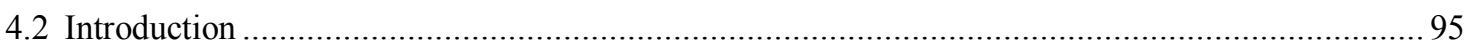

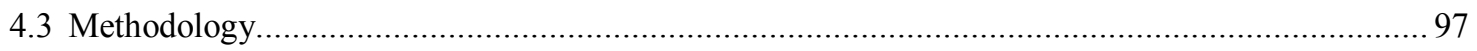

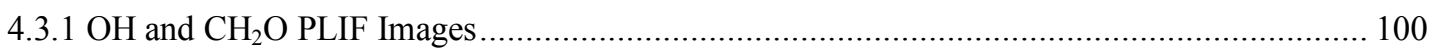

4.3.2 Local Peak Heat Release Rate, Flame Thickness, and Curvature......................................... 100

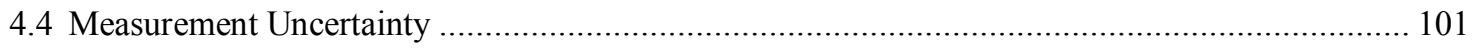

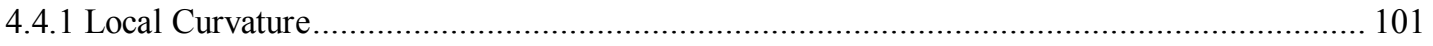

4.4.2 Local Peak Heat Release Rate and Flame Thickness............................................................ 103

4.4.3 Ensemble Average of Peak HRR and Flame Thickness ....................................................... 106

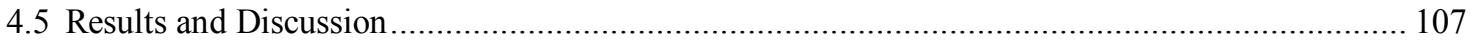

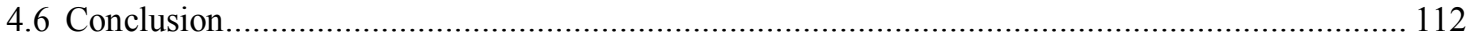

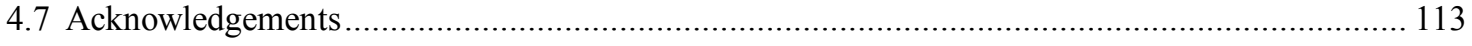

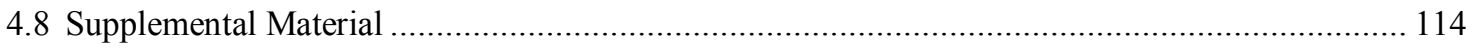

4.8.1 Further Explanation of "Front-Supported" and "Back-Supported" Flame Configurations ....... 114

4.8.2 Details on the Inputs of the Monte Carlo Uncertainty Simulations of Local Curvature, Peak

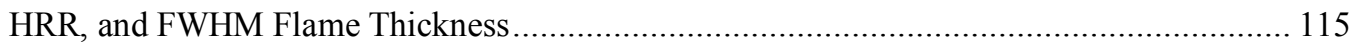

Chapter 5 Conclusions and Recommendations ......................................................................................... 116 


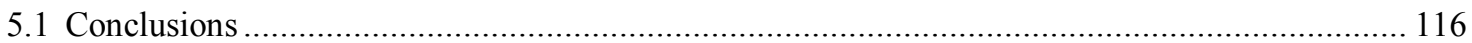

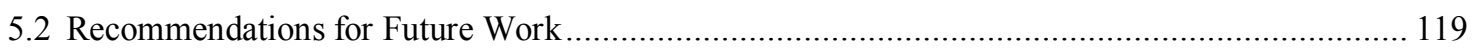

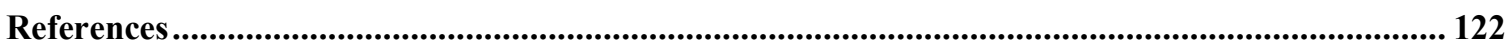

Appendix A Determination of the Effective Spatial Resolution of the Optical Imaging System...... 129

Appendix B Supplemental Photographs, Manufacturing Drawings, and Schematics of the Experimental Apparatus, NRC Stratified Slot Burner, and Iso-Octane/Air Delivery System ..... 131

B.1 Detailed Photographs of the Experimental Apparatus................................................................... 132

B.2 Manufacturing Drawings of the NRC Stratified Slot Burner.................................................... 135

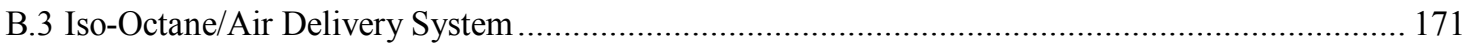

Appendix C Further Details of Processing of Individual 3-Pentanone, $\mathrm{OH}$, and $\mathrm{CH}_{2} \mathrm{O}$ PLIF Images

C.1 Configuration and Calibration Procedures for PLIF Experiments ............................................... 172

C.2 Image Processing Flow Charts and Sample Intermediate Images for 3-Pentanone, $\mathrm{OH}$, and $\mathrm{CH}_{2} \mathrm{O}$

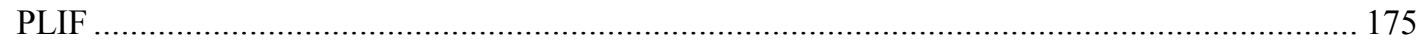




\section{List of Tables}

Table 1.1 Summary of literature examining the effects of stratification on the local flame speed and flammability limit. 7

Table 1.2 Summary of literature examining the effects of stratification on flame structure and topology. . 14

Table 1.3 Summary of literature examining the effects of stratification on both the local and global reaction rate. 28

Table 3.1 Test conditions and reactant properties. 66

Table 3.2 Total flame length and heat release rate per instantaneous image. .86

Table 4.1 Summary of test conditions. 99

Table 4.2 Bias error of ensemble average of local peak HRR and instantaneous flame thickness $\delta_{t}$ due to combined effects of the differing range of $\phi$ within the ROI and the temperature dependence of the $\mathrm{OH}$ and $\mathrm{CH}_{2} \mathrm{O}$ fluorescence signals. 107

Table 4.3 Peak HRR fluorescence intensity and FWHM profile thickness $\delta_{\mathrm{t}}$

Table 4.4 Summary of input distributions used for the Monte Carlo simulations of the uncertainty of local curvature, peak HRR, and FWHM flames thickness along an instantaneous flame realization.... 115 


\section{List of Figures}

Figure 2.1 Reactant mixture progression through (1) lower, (2a,b) lateral, and (3) transverse mixing sections of the stratified burner.

Figure 2.2 PLIF setup for simultaneous $\mathrm{OH}$ and $\mathrm{CH}_{2} \mathrm{O}$ acquisition

Figure 2.3 Transverse variation in equivalence ratio for five flame conditions with the gradient expressed in terms of turbulent flame brush thickness $\mathrm{L}_{\mathrm{t}}$.

Figure 2.4 Mixture strength distribution and corresponding interrogation window for stratified flame $\mathrm{L}_{\mathrm{t}} \delta \phi / \delta \mathrm{y}=0.22$.. 46

Figure 2.5 Progress variable $\langle\mathrm{c}\rangle$ map and corresponding interrogation window for stratified flame $\mathrm{L}_{\mathrm{t}} \delta \phi / \delta \mathrm{y}=0.16$ 48

Figure 2.6 Flame surface density for premixed and stratified flames.

Figure 2.7 Comparison of curvature PDFs for globally stoichiometric premixed and partially-premixed isooctane/air V-flames. 50

Figure 3.1 Reactant mixture progression through (1) lower, (2a, 2b) lateral, and (3) transverse mixing sections of the stratified burner. A sample instantaneous equivalence ratio image is shown between axial positions $\mathrm{x}=0-120 \mathrm{~mm}$ above the exit plane of the burner for stratified flame condition $\delta \phi / \delta y=0.021 \mathrm{~mm}^{-1}$, where the white rectangle identifies the region between $\mathrm{x}=31$ $71 \mathrm{~mm}$ where $\mathrm{OH}$ and $\mathrm{CH}_{2} \mathrm{O}$ PLIF images were acquired.

Figure 3.2 Planar laser induced fluorescence setup for simultaneous $\mathrm{OH}$ and $\mathrm{CH}_{2} \mathrm{O}$ acquisition......

Figure 3.3 Transverse profiles of velocity $\diamond$ and turbulence intensity $\diamond$ in the burner exit plane at axial position $\mathrm{x}=5 \mathrm{~mm}$.

Figure 3.4 Close-up highlighting the decrease in signal intensity along leading edge of 3-pentanone front in (a) for non-eroded and in (b) for eroded image for stratified flame condition $\delta \phi / \delta y=0.021 \mathrm{~mm}^{-1} .64$

Figure 3.5 Example equivalence ratio contours derived from 3-pentanone fluorescence images for stratified flame setting $\delta \phi / \delta y=0.021 \mathrm{~mm}^{-1}$ showing (a) instantaneous and (b) average conditioned images.

Figure 3.6 Normalized profiles of $[\mathrm{OH}],\left[\mathrm{CH}_{2} \mathrm{O}\right],[\mathrm{HCO}],[\mathrm{OH}][\mathrm{CH} 2 \mathrm{O}], \mathrm{k}[\mathrm{OH}]\left[\mathrm{CH}_{2} \mathrm{O}\right], \mathrm{HRR}, \mathrm{f}_{\mathrm{OH}}[\mathrm{OH}]$, $\mathrm{f}_{\mathrm{CH} 2 \mathrm{O}, \mathrm{T}}-0.5\left[\mathrm{CH}_{2} \mathrm{O}\right], \mathrm{f}_{\mathrm{CH} 2 \mathrm{O}, \mathrm{T}^{-}}-0\left[\mathrm{CH}_{2} \mathrm{O}\right], \mathrm{f}_{\mathrm{OH}}[\mathrm{OH}] \mathrm{f}_{\mathrm{CH} 2 \mathrm{O}, \mathrm{T}^{-}}-0.5\left[\mathrm{CH}_{2} \mathrm{O}\right], \mathrm{f}_{\mathrm{OH}}[\mathrm{OH}] \mathrm{f}_{\mathrm{CH} 2 \mathrm{O}, \mathrm{T}^{-}}-0\left[\mathrm{CH}_{2} \mathrm{O}\right]$, and Temperature for (a) $\phi=0.75$, (b) $\phi=1.00$, and (c) $\phi=1.25$. 70

Figure 3.7 Correlation of $(\mathrm{OH}$ LIF $)\left(\mathrm{CH}_{2} \mathrm{O}\right.$ LIF) with heat release rate for $\mathrm{CH}_{2} \mathrm{O}$ quenching models $\mathrm{T}^{-0.5}$ in (a) and $\mathrm{T}^{-1.0}$ in (b) for simulated premixed flames ranging from $\phi=0.75-1.25$. Variation of the ratio of integrated and peak $(\mathrm{OH} L I F)\left(\mathrm{CH}_{2} \mathrm{O} \mathrm{LIF}\right) / \mathrm{HRR}$ over a range of equivalence ratios is presented in (c).

Figure 3.8 Instantaneous flame front position from heat release rate contour and product side of $\mathrm{CH}_{2} \mathrm{O}$ contour by image segmentation for stratified flame condition $\partial \phi / \partial y=0.029 \mathrm{~mm}^{-1}$. . . 
Figure 3.9 Instantaneous PLIF images of $\mathrm{OH}$ (left), $\mathrm{CH}_{2} \mathrm{O}$ (centre), and relative heat release rate (right) for different equivalence ratio gradients. 78

Figure 3.10 Sample instantaneous heat release rate contour for premixed flame condition $\delta \phi \delta y=-$ $0.001 \mathrm{~mm}^{-1}$. Boxed region in (a) corresponds to the zoomed region shown in (b). White dotted lines in (b) indicate equally spaced locally normal profiles along the leading edge of the HRR contour. The peak HRR value from each profile was matched to the calculated curvature value at the leading edge.

Figure 3.11 Heat release rate vs. curvature for reference fully-premixed and stratified flame conditions with matching region of interest.

Figure 3.12 PDF of HRR fluorescence intensity for all gradient cases.

Figure 3.13 Windowed (a) heat release rate fluorescence intensities and (b) flame surface density as a function of progress variable $\langle c\rangle$ for reference premixed and stratified flames.

Figure 3.14 Experimental laminar flame speed $S_{L}$ [95-99], polynomial fit to experimental $S_{L}$ data, and simulated peak heat release rate for premixed flames ranging from $\phi=0.75-1.25$.

Figure 3.15 Weighted linear correlation function of fluorescence intensity (measured in pixel counts) to equivalence ratio and upper/lower $95 \%$ confidence intervals.

Figure 3.16 Correlation of $(\mathrm{OH} \mathrm{LIF})\left(\mathrm{CH}_{2} \mathrm{O}\right.$ LIF) with the forward reaction rate $\mathrm{k}[\mathrm{OH}]\left[\mathrm{CH}_{2} \mathrm{O}\right]$ for $\mathrm{CH}_{2} \mathrm{O}$ quenching models $\mathrm{T}^{-0.5}$ in (a) and $\mathrm{T}^{-1.0}$ in (b) for simulated premixed flames ranging from $\phi=0.75$ 1.25. Variation of the ratio of integrated and peak $\left(\mathrm{OH} \mathrm{LIF)}\left(\mathrm{CH}_{2} \mathrm{O}\right.\right.$ LIF) / $\mathrm{k}[\mathrm{OH}]\left[\mathrm{CH}_{2} \mathrm{O}\right]$ over a range of equivalence ratios is presented in (c).

Figure 3.17 Heat release rate vs. curvature for reference premixed and stratified flame conditions plotted without using the windowing technique.

Figure 3.18 Schematic illustrating the anticipated effect of mean $\phi$ gradients on thermal back support (heat flux) to and preferential diffusion (heat and species flux) away from near stoichiometric flame regions $0.95 \leq \phi \leq 1.05$ within a ROI along a stratified flame front as the gradient steepens from (a) to (b).

Figure 4.1 PLIF setup for simultaneous $\mathrm{OH}$ and $\mathrm{CH}_{2} \mathrm{O}$ acquisition. .98

Figure 4.2 Quantification of curvature uncertainty. In (a) open circles show spatially jittered flame positions for three sample runs of the Monte Carlo analysis, while sample histograms show distributions of local curvature and $95 \%$ confidence limits evaluated at three different sample positions along the flame. Final computed $95 \%$ confidence limits for total and relative curvature uncertainty in (b).

Figure $4.395 \%$ confidence intervals for the relative uncertainty of the local peak HRR (a) and the local flame thickness $\delta_{\mathrm{t}}(\mathrm{b})$.

Figure 4.4 Example normalized profiles of (OH LIF) and $\left(\mathrm{CH}_{2} \mathrm{O}\right.$ LIF) fluorescence intensity and (OH LIF)( $\mathrm{CH}_{2} \mathrm{O}$ LIF) HRR at a position along an instantaneous realization of the globally rich, stratified flame condition at $\delta \phi / \delta y=0.012 \mathrm{~mm}^{-1}$.

Figure 4.5 Mean HRR and instantaneous flame thickness $\delta_{t}$ vs curvature for $(a, d)$ rich front- and backsupported, $(\mathrm{b}, \mathrm{e})$ near-stoichiometric, and (c,f) lean back-supported premixed and stratified flame conditions.

Figure 4.6 Schematic highlighting the variation in $\phi$ for flame regions adjacent to the ROI for "frontsupported" and "back-supported" V-flames. 


\section{Nomenclature}

Latin

Description

First Use

Symbol

Ensemble average of the enclosed quantity

Units

$\mathrm{Pg}$.

$\mathrm{A} / \mathrm{F}$

a.u.

Air-to-fuel ratio

[-] 4

c

Arbitrary units

$[-] \quad 78$

f

Reaction progress variable

$[-] \quad 47$

global

Temperature dependence of the fluorescence signal

$[-]$

68

$\mathrm{k}$

On the scale of the entire flame system or overall measurement domain

Chapter 2: experimentally derived constant used for chemical

$[-]$

closure models for fractal flamelets

Chapter 3: forward rate constant of

$\mathrm{OH}+\mathrm{CH}_{2} \mathrm{O} \rightarrow \mathrm{HCO}+\mathrm{H}_{2} \mathrm{O}$ reaction

Le Lewis number defined as the ratio of the thermal diffusivity of [-]

$\left[\mathrm{L} \cdot \mathrm{mol}^{-1} \cdot \mathrm{s}^{-1}\right] \quad 68$

the reactant mixture to the molecular diffusivity of the

deficient reactant

local

On the scale of the instantaneous flame thickness

$\begin{array}{lr} & 3 \\ {[\mathrm{~mm}]} & 45 \\ {[-]} & 45 \\ {\left[\mathrm{~cm}^{3} \cdot \mathrm{s}^{-1}\right]} & 69 \\ {[-]} & 21 \\ {[-]} & 20 \\ {\left[\mathrm{~cm} \cdot \mathrm{s}^{-1}\right]} & 24 \\ {\left[\mathrm{~cm} \cdot \mathrm{s}^{-1}\right]} & 5 \\ {\left[{ }^{\circ} \mathrm{C} \mathrm{or} \mathrm{K}\right]} & 68 \\ {\left[\mathrm{~m} \cdot \mathrm{s}^{-1}\right]} & 14 \\ {\left[\mathrm{~m} \cdot \mathrm{s}^{-1}\right]} & 14 \\ {[\mathrm{~m}]} & 41\end{array}$

$\mathrm{L}_{\mathrm{t}} \delta \phi / \delta \mathrm{y}$

Turbulent flame brush thickness

Equivalence ratio gradient

Quenching rate

$\mathrm{Q}_{21}$

Reynold's number

S Swirl number

$\mathrm{S}_{\mathrm{L}}^{\mathrm{o}} \quad$ Unstrained laminar flame speed

$\mathrm{S}_{\mathrm{L}} \quad$ Laminar flame speed

$\mathrm{T} \quad$ Temperature

$\overline{\mathrm{U}}, \overline{\mathrm{V}}, \overline{\mathrm{W}}$

Mean components of velocity in $\mathrm{x}, \mathrm{y}$, and $\mathrm{z}$ directions

u', v', w'

Fluctuating components of velocity in $\mathrm{x}, \mathrm{y}$, and $\mathrm{z}$ directions

Axial, transverse, and lateral directions of the coordinate

system

Greek

Description

Kronecker delta

Units

First Use

Symbol

Instantaneous flame front position

$\delta\left(\mathrm{c}-\mathrm{c}_{\mathrm{f}}\right)$

$\delta_{\mathrm{t}}$

Instantaneous flame thickness

$\delta \phi / \delta y \quad$ Equivalence ratio gradient

Kolmogorov length scale

Curvature

Taylor length scale

Integral length scale of turbulence

Mean signal intensity

Chapter 3: quenching cross section

Chapters 3 and 4: standard deviation

$\Sigma \quad \quad$ Flame surface density

$\Sigma$ Instantaneous flame surface density

Equivalence ratio

Mean equivalence ratio

$[-]$

$[-]$

$\left[\mathrm{mm}^{-1}\right]$

$[\mathrm{mm}]$

$\left[\mathrm{mm}^{-1}\right]$

$[\mathrm{mm}]$

[mm]

[a.u.]

$\left[\AA^{2}\right]$

[-]

$\left[\mathrm{mm}^{-1}\right]$

$\left[\mathrm{mm}^{-1}\right]$

[-]

$[-]$

$[-]$ 


\begin{tabular}{|c|c|c|}
\hline Subscripts & Description & $\begin{array}{r}\text { First Use } \\
\text { Pg. }\end{array}$ \\
\hline 1 or 2 & Indicating a particular reactant inlet line to the stratified burner & 42 \\
\hline$a$ and $b$ & $\begin{array}{l}\text { Indicating a particular reactant mixing section of the stratified } \\
\text { burner }\end{array}$ & 42 \\
\hline background & Referring to the background signal & 61 \\
\hline fluorescence & Referring to the fluorescence signal & 61 \\
\hline $\mathrm{i}$ & $\begin{array}{l}\text { Referring to initial Cartesian coordinates along an instantaneous } \\
\text { flame front }\end{array}$ & 102 \\
\hline $\mathrm{i}, \mathrm{MC}$ & $\begin{array}{l}\text { Referring to the jittered Cartesian coordinates along an } \\
\text { instantaneous flame front }\end{array}$ & 102 \\
\hline $\mathrm{i}, \mathrm{ref}$ & $\begin{array}{l}\text { Referring to the Cartesian coordinates along a reference } \\
\text { instantaneous flame front }\end{array}$ & 102 \\
\hline PLIF & $\begin{array}{l}\text { Referring to raw fluorescence of stratified flame settings along } \\
\text { equivalent } \phi \text { iso-contours within reactants }\end{array}$ & 67 \\
\hline Ref, premixed & $\begin{array}{l}\text { Referring to raw fluorescence of reference-premixed flame } \\
\text { settings along equivalent } \phi \text { iso-contours within reactants }\end{array}$ & 66 \\
\hline strat and ref & Referring to stratified and reference-premixed flame settings & 66 \\
\hline$\phi$ & $\begin{array}{l}\text { Referring to estimated standard deviation of } \phi \text { along equivalent } \phi \\
\text { iso-contours within reactants }\end{array}$ & 66 \\
\hline Chemical Species & Description & $\begin{array}{r}\text { First Use } \\
\text { Pg. }\end{array}$ \\
\hline $\mathrm{C}_{2} \mathrm{H}_{5} \mathrm{COC}_{2} \mathrm{H}_{5}$ & 3-pentanone & 42 \\
\hline $\mathrm{C}_{3} \mathrm{H}_{8}$ & Propane & 14 \\
\hline $\mathrm{C}_{8} \mathrm{H}_{18}$ & Iso-octane, (i.e. 2,2,4-trimethylpentane, $\left.\left(\mathrm{CH}_{3}\right)_{3} \mathrm{CCH}_{2} \mathrm{CH}\left(\mathrm{CH}_{3}\right)_{2}\right)$ & 19 \\
\hline $\mathrm{CH}^{*}$ & $\mathrm{CH}$ intermediate in electronically excited state & 15 \\
\hline $\mathrm{CH}_{2} \mathrm{O}$ & Formaldehyde & 19 \\
\hline $\mathrm{CH}_{3} \mathrm{COCOCH}_{3}$ & Biacetyl & 59 \\
\hline $\mathrm{CH}_{4}$ & Methane & 7 \\
\hline $\mathrm{CO}$ & Carbon monoxide & 11 \\
\hline $\mathrm{CO}_{2}$ & Carbon dioxide & 4 \\
\hline $\mathrm{H}_{2}$ & Hydrogen & 13 \\
\hline $\mathrm{H}_{2} \mathrm{O}$ & Water & 18 \\
\hline $\mathrm{HCO}$ & Formyl radical & 68 \\
\hline $\mathrm{N}_{2}$ & Nitrogen & 18 \\
\hline Nd:YAG & Neodymium-doped yttrium aluminum garnet & 43 \\
\hline $\mathrm{NO}_{2}$ & Nitrogen dioxide & 14 \\
\hline NOx & Oxides of nitrogen & 4 \\
\hline $\mathrm{O}_{2}$ & Oxygen & 18 \\
\hline $\mathrm{OH}^{*}$ & $\mathrm{OH}$ intermediate in electronically excited state & 15 \\
\hline $\mathrm{OH}$ & Hydroxyl radical & 11 \\
\hline $\mathrm{ZrO}_{2}$ & Zirconium dioxide & 17 \\
\hline
\end{tabular}


First Use

\begin{tabular}{llr} 
Acronyms & Description & Pg. \\
\hline 1D, 2D, 3D & One-, two-, and three-dimensional & 7 \\
CI & Confidence intervals & 102 \\
DISI & Direct injection spark ignition & 4 \\
DNS & Direct numerical simulation & 24 \\
FSD & Flame surface density & 11 \\
FWHM & Full width at half maximum & 101 \\
HP & Referring to a high-pass filter & 59 \\
HRR & Heat release rate & 24 \\
ICCD & Intensified charge-coupled device & 59 \\
IR & Infra red & 43 \\
LDA & Laser Doppler anemometry & 17 \\
LDV & Laser Doppler velocimetry & 12 \\
LES & Large eddie simulation & 12 \\
LIF & Laser induced fluorescence & 34 \\
loess & Locally-weighted linear regression & 101 \\
LSF & Line spread function & 129 \\
MC & Monte Carlo & 34 \\
NRC & National Research Council of Canada & 19 \\
OTF & Optical transfer function & 129 \\
PDF & Probability density function & 10 \\
PIV & Particle image velocimetry & 12 \\
PLIF & Planar laser induced fluorescence & 8 \\
RMS & Root mean squared & 14 \\
rloess & Robust locally-weighted linear regression & 105 \\
ROI & Region of interest & 12 \\
SAE & Society of Automotive Engineers & 34 \\
SNR & Signal to noise ratio & 98 \\
SPIV & Stereoscopic particle image velocimetry & 12 \\
SRF & Step response function & 129
\end{tabular}




\section{Preface}

This integrated thesis is composed of three articles that have been published, submitted, or will be submitted to scholarly journals:

Vena, P.C., Deschamps B., Smallwood, G.J., Johnson, M.R. "Equivalence ratio gradient effects on flame front topology in a stratified iso-octane/air turbulent V-flame", Proceedings of the Combustion Institute, 33 (2011): 1551:1558. DOI: 10.1016/j.proci.2010.06.041.

Vena, P.C., Deschamps B., Guo, H., Smallwood, G.J., Johnson, M.R. "Heat release rate variations in a globally stoichiometric, stratified iso-octane/air turbulent V-flame", submitted to Combustion and Flame and under consideration for publication (submitted December 13, 2013; manuscript id: CNF-D-13-00630).

Vena, P.C., Deschamps B., Johnson, M.R. "A comparative analysis on the effects of stratification on locally lean, near-stoichiometric, and rich iso-octane/air turbulent V-flames", to be submitted to Combustion and Flame.

All three articles are included in their entirety; the only noteworthy changes to the manuscripts are adjustments to the nomenclature and to the numbering of equations, figures, tables, and references to ensure consistency throughout the thesis, as well as minor edits resulting from the examiner's comments during the defence.

Since the first article was published in the Proceedings of The Combustion Institute, its copyright is attributed to the publisher, Elsevier. Permissions to reproduce this work with minor edits are included in supporting documents. Copyright for the second and third articles is held by the authors since they have not yet been published.

All three articles provide detailed accounts of the experimental procedures followed to obtain results, and are considered sufficient to allow readers to test the validity of research outcomes. For this reason the thesis does not contain a separate "Methods Chapter" as described in $\S 12.4 \mathrm{~B}$ of the 2013-2014 Carleton University Graduate Calendar.

The preferred method of citing work from this thesis is to directly reference the published articles when possible, citing this thesis only when an appropriate citation in the included articles cannot be found. 


\section{Student Statement Regarding Student Involvement}

I, Patrizio Vena, was fully involved and responsible for setting up equipment, obtaining data, analyzing results, and writing the first drafts of the material presented in the accompanying thesis.

Patrizio Christian Vena

\section{Supervisor Statement Regarding Student Involvement}

The student, Patrizio Vena, was fully involved and responsible for setting up equipment, obtaining data, analyzing results, and writing the first drafts of the material presented in the accompanying thesis.

Matthew Ronald Johnson 


\section{Chapter 1}

\section{Introduction}

\subsection{Background and Literature Review}

Fossil fuels are and will remain a primary energy source for the foreseeable future, particularly for transportation purposes [1]. Although a range of alternative technologies such as fuel cell and electric power continue to be developed [2], none has demonstrated the energy density and storage capability of fossil fuels. It is therefore necessary to further develop the practical and fundamental understanding of combustion processes as a prerequisite to pollutant mitigation and environmental sustainability.

Flames can be defined as self-sustaining waves driven by a series of exothermic chemical reactions between a fuel and oxidizer. Traditionally, they are classified into two broad categories depending on the mixedness of the reactants prior to ignition. In non-premixed, or diffusion flames, the fuel and oxidizer are initially separated and meet at the reaction front, where reactions occur simultaneously with the continuous interdiffusion of species. In premixed flames, the reactants are fully mixed at the molecular level before any significant chemical reactions take place. If this homogenous mixture of fuel and oxidizer is ignited, and if the mixture is flammable (i.e. the fuel to oxidizer ratio lies within flammability limits of the reactants for the relevant pressure and temperature), a flame propagates through the mixture. The propagating flame divides the combustion system into two stable states, as it consumes unburned reactants and ejects burnt products [3].

The classical definitions of premixed and non-premixed flames represent extreme cases, and in several practical devices, flames operate within an intermediate regime in which reactants are partially premixed, or stratified. In this case, the flame propagates through a reactant mixture where stoichiometry varies locally, altering both the global (i.e. on the scale of the entire flame system or overall measurement domain) behaviour of the combustion system, and the local (i.e. on the scale of the instantaneous flame 
thickness) properties of the reaction zone along the flame front. This is the case is new generation, stratified charge, Direct Injection Spark Ignition (DISI) engines, where the fuel is directly injected into the combustion chamber, and the reactant mixture is ignited as fuel and oxidizer continue to mix. This results in the presence of severe spatial variations in the $\mathrm{A} / \mathrm{F}$ ratio, where both large- and small-scale stratification occurs simultaneously. While small-scale variations in $\mathrm{A} / \mathrm{F}$ also arise due to turbulence and incomplete liquid-fuel vaporization, large-scale spatial gradients are utilized as a means of reducing pollutant emissions and fuel consumption [4].

The stratified combustion approach has been shown to result in higher mean effective pressure $[5,6]$ and thermal efficiency [6], with lower flammability limits than in the homogeneous case of the same equivalence ratio $[7,8]$. By limiting misfire or slow burning, reductions in fuel consumption and pollutant emissions including particulates such as soot, oxides of nitrogen (NOx), and greenhouse gases like carbon dioxide $\left(\mathrm{CO}_{2}\right)$ (via reduced fuel consumption) have been achieved [9-12] and significant gains in engine performance obtained [13-15].

Among the first detailed examinations on stratified combustion were those of Cho and Santavicca [16], and Zhou et al. [17], who specifically considered the effect of reactant heterogeneity (the magnitude of spatial and temporal fluctuations in mixture strength) on flame properties. They concluded that the degree and scale of non-homogeneity affects global combustion properties and alters local flame characteristics when neighbouring pockets of different reactant concentration interact. The latter observation had significant implications, as it suggested that the expected behaviour of local flame regions, or flamelets, could not be predicted solely from reactant properties, contrary to generally accepted flamelet theory, on which many numerical models are based.

Flamelet models rely on the notion that, at high Damkhöler numbers, a turbulent flame can be approximated as an amalgamation of asymptotically thin, laminar flamelets [18] that may be stretched and/or strained by turbulent structures in a flow field [19,20]. This implies that for a given set of turbulence characteristics and scalar reactant properties, the behaviour of premixed flames can be predicted, and specifically assumes that interactions between neighbouring flame regions are negligible, an 
assumption the studies of Cho and Santavicca [16], and Zhou et al. [17], and other recent studies may invalidate.

Despite the importance of partial premixing for practical combustion devices and potential advantages of optimizing stratified charge engines, few fundamental works have attempted to quantify the effects of stratification on the local properties of turbulent flames. The difficulty lies in implementing an experiment capable of precisely reproducing partial premixing and coupling it with the necessary diagnostics to evaluate these complex flames. Only recently, with the advent of novel optical diagnostics, has this been a realistic proposition. The development of practical high energy lasers, adequate data acquisition systems, and the necessary image processing tools have led to the non-intrusive visualization techniques necessary to accurately probe the physical and chemical phenomena in turbulent partially premixed flames.

Despite sometimes conflicting results, the three most notable, generally accepted effects of stratification on flame behaviour are: (1) variations in local flame speed and flammability limit, (2) the change in flame structure and topology, and (3) the variation in both the local and global reaction rate. A broad overview of each subject will be presented here with discussion of how the current work fits into the published literature. Tables summarizing the relevant literature are also included. Targeted literature reviews are also contained in each of the three articles presented in this thesis.

\subsubsection{Variations in Local Flame Speed and Flammability Limit}

To isolate the effects of stratification on the local behaviour of the reaction zone from those of simple variations in mixture strength, flame configurations have been devised in which the flame front propagates solely in the direction of the gradient vector in equivalence ratio. By precisely controlling the mixture gradient normal to a laminar flame front in freely propagating [21] and channel-flow [22-26] type flames, the behaviour of stratified flames can be quantified. Reactant mixtures can then be adjusted to study locally rich, stoichiometric, and lean flames, and this, at various levels of stratification (i.e $\phi$ gradients of varying steepness). These studies are primarily focused on characterising variations in local flame speed $\mathrm{S}_{\mathrm{L}}$ and flammability limits, and a summary of the major contributions to the literature is provided in Table 1.1. 
It has been observed that as a flame propagates from rich to lean mixtures, heat and radicals from the richer products feed the leaner flame region, and enhance its resistance to extinction. This is often referred to as "back support" or a "memory effect" $[21-24,26]$ in that combustion, and more specifically the laminar flame speed, is not determined strictly by the reactant properties as would be suggested by the flamelet model, but also by the composition and temperature of the products.

This implies that both the local $S_{L}$ and the reaction rate are not strictly a function of reactant properties, but also of the composition and temperature of the products. Kang and Kyritsis [22-24] argue that the greater heat generated from mixtures of higher mixture strength that are downstream of the flame front compensate for the upstream decrease in equivalence ratio, resulting in increased flame speed. The numerical work of Pires Da Cruz [26] supported the experimental findings of Ra and Cheng [21] and Kang and Kyritsis [22-24], and concluded that the increase in $S_{L}$ and extension of the lower flammability limit were caused by the high temperature of products behind the reacting front.

Conversely, for cases in which a flame propagates from leaner to richer mixtures, Kang and Kyritsis [25] found similar flame behaviour to that discussed above. They reported that in flames propagating from stoichiometric to rich mixtures, $\mathrm{S}_{\mathrm{L}}$ also increases because of the heat originating from higher downstream flame temperature. They further suggested that the resistance to extinction was enhanced by hydrogen generation. Pires Da Cruz et al. [26] confirmed that the production and consumption of molecular hydrogen controlled the propagation of flames in a rich stratified mixture; however, their results conflicted with those of Kang and Kyritsis [25] in that stoichiometric to rich flames would slow down, as opposed to rich to stoichiometric flames that accelerate. 
Table 1.1 Summary of literature examining the effects of stratification on the local flame speed and flammability limit.

Freely expanding and pipe-flow type flames propagating solely in the direction of the gradient vector in equivalence ratio

\section{Pires Da Cruz et al. (2000) [26], numerical}

- $1 \mathrm{D}$ adiabatic $\mathrm{CH}_{4} /$ air flame propagating at $300 \mathrm{~K}$ and $100 \mathrm{kPa}$ in $0.1 \mathrm{~m}$ pipe closed at the left end and open at the right with the flame moving from left to right

- initial step functions in equivalence ratio -

- once the flame has reached position $\mathrm{x}=0.05 \mathrm{~m}$, the step function has settle

- flame position fixed at $\mathrm{x}=0.05 \mathrm{~m}$, reactants into a gradient from $\mathrm{x}=0.00 \mathrm{~m}$ to $0.05 \mathrm{~m}$ and products

from $\mathrm{x}=0.05 \mathrm{~m}$ to $\mathrm{x}=0.10 \mathrm{~m}$

- 4 test cases: $\phi=1.0-0.35, \phi=1.0-2.5$,

$\phi=1.5-1.0$, and $\phi=1.0-2.5-0.0-1.0$
-1D simulation with Lawrence Livermore National Laboratory HTC code [27]

- reduced GRI 2.11 mechanism with 177 reactions and 32 species
- stoich to lean mixtures: transient flame propagates faster than corresponding homogeneous flame

- stoich to rich mixtures: flame speed decreased

- rich to stoich mixtures: flame speed increased

\section{Ra and Cheng (2001) [21], experimental and numerical}

- freely expanding $\mathrm{CH}_{4} /$ air flame at constant pressure and ignited with focused laser beam

- flames propagated from richer to leaner mixtures, $\phi_{1}=0.55: 1.00$ and $\phi_{2}=0.55: 1.00$
- soap bubble to evaluate flame propagation across step function distribution in equivalence ratio

- initial stratification was achieved by filling centrally located bubble of mixture $\phi_{1}$ surrounded by mixture $\phi_{2}$

- ignited centrally located $\phi_{1}$ mixture with a typical diameter of $15-25 \mathrm{~mm}$ and a volume ratio of charge $\phi_{1}$ to $\phi_{2}$ of $\sim 0.1$ to $0.4 \%$
- flame propagation captured with high speed camera for Schlieren imaging

- multiple mixture combinations attempted

-1D simulation with reduced chemistry, 24 species and 57 reactions [28] flame speed to relax from steady state value in $\phi_{1}$ to that of $\phi_{2}$

- simulation in good agreement with experimental results 
Table 1.1 Summary of literature examining the effects of stratification on the local flame speed and flammability limit. (continued)

flame configuration and flow settings approach to stratification analysis methodology observations and results

\section{Kang and Kyritsis (2005, 2007a, 2007b) [22-24], experimental}

- near planar, spark ignited $\mathrm{CH}_{4} /$ air flame propagating through a $9 \mathrm{~cm}$ section with a $20 \mathrm{~mm} \times 20 \mathrm{~mm}$ cross-section at constant pressure

- flames ignited under near stoich conditions and propagated to gradually leaner mixtures, $\phi \approx 1.0-0.2$

- quartz windows allowed optical access to combustion chamber
- convective-diffusive balance to establish stratification in a combustion chamber

- mixture $\phi_{1}$ introduced over top of chamber, allowed to diffuse through two wire screens and mix with the second mixture $\phi_{2}$ to form controllable equivalence ratio gradient zone controllable equivalence
several centimeters thick
- acetone PLIF used to measure cold flow stratification and equivalence ratio

- propagation velocity measured using a Phantom V7.0 high speed camera at 1000 frames per second

- theoretical arguments with activation energy asymptotics suggested the controlling parameter in stratified flames
- stoich to lean: higher local flame speeds in stratified mixtures compared to

corresponding homogeneous cases, particularly in mixtures near lower flammability limit for $0.5<\phi<0.7$

- stoich to lean: extension of the lower flammability limit, flame can penetrate to $\phi \approx 0.35$, whereas in a homogeneous mixture, no flame can be ignited for $\phi \approx<0.58$

\section{Kang and Kyritsis (2009) [25], experimental}

- near planar spark ignited $\mathrm{CH}_{4} /$ air flame as in [22-24]

- flames propagating from stoich $\phi \approx 1.00$ to

rich mixtures $\phi \approx 1.52,2.28,3.04,3.8$

- near stagnant reactant conditions with counterflow speeds ranging from 0.1 $0.7 \mathrm{~cm} / \mathrm{s}$
- mixtures $\phi_{1}$ and $\phi_{2}$ were introduced at opposite ends of the chamber in counterflow, based on Stefan flow
- line-Raman imaging measured the $\mathrm{CH}_{4}$ concentration in cold mixture

flame speed measured using same approach as [22-24]
- stoich to rich: increased flame speed in most stratified mixtures compared to the corresponding homogeneous cases

- stoich to rich: extension of the upper flammability limit 


\subsubsection{Change in Flame Structure and Topology}

Academic studies on the effects of stratification in turbulent flames have been conducted on freely propagating flames, V-flames, axisymmetric flames, and swirl burners. Variations in flame structure and topology with stratification have been observed in terms of flame thickness and rate of strain [29-38]; flame length and wrinkling scales $[4,16,17,29,30,39-46]$; flame surface density $[31,34,45,47,48]$; and curvature $[29-35,41,45-47]$. The major contributions to the literature are summarized in Table 1.2 and subdivided based on flame configuration.

Cho and Santaviccia [16] and Zhou et al. [17] were among the first to conduct an experimental evaluation of the effects of partial premixing on flame behaviour, studying spark-ignited propane/air mixtures in which the flame propagated in a constant volume combustion chamber. They measured variations in mean flame propagation velocity, wrinkling ratio, and pressure at varying levels of reactant heterogeneity, turbulence intensity, and global equivalence ratio. Their results suggested that small-scale stratification generally enhances flame wrinkling, resulting in a more corrugated front with increased flame surface area relative to the homogeneous case. Zhou et al. [17] further observed monotonic flame surface generation with stratification and a maximum mean flame propagation velocity at what they suggested was an optimal level of stratification.

The experimental results of Renou et al. [41] on freely propagating propane/air flames support the trends observed by Cho and Santaviccia [16] and Zhou et al. [17]. They reported a significantly greater distribution in curvature, including increased amounts of sharp negative (concave towards the reactants) and positive (convex towards the reactants) curvatures with characteristic lengths comparable to those of the local fluctuations in mixture strength. Experimental observations by Samson [49] also showed an increase in flame length due to local $\phi$ inhomogeneities. They further observed that small-scale variations in mixture strength lead to curvatures of comparable length scales with a greater distribution.

Pasquier et al. [4] reported an increase in wrinkling with stratification, observing local variations in burning velocity along the flame front. They proposed that the distribution of fuel rich and/or fuel lean pockets can either increase or decrease the local flame propagation velocity. Indeed, in the presence of a greater concentration of locally stoichiometric pockets, the propagation of lean pockets is enhanced due to 
higher product temperatures. Conversely, the local propagation of locally stoichiometric pockets may be reduced if surrounded by a majority of lean pockets due to a decrease in temperature. Evidence of back support from large-scale gradients was also reported by Pasquier et al. [4]. Stratified flames propagating from near stoichiometric to leaner conditions showed a greater mean propagation speed than the homogeneous flame of the same global equivalence ratio. Results were in agreement with those of Pires da Cruz [26], Ra and Cheng [21], and Kang and Kyritsis [22-24].

Experiments on rod-stabilized V-flames $[29-31,36,38,42-45,47,48,50]$ have shown a change in flame structure and general increase in wrinkling for flames stabilized in transverse gradients in equivalence ratio. Robin et al. [29] concluded that the effect of stratification-induced stretch was nonnegligible and observed a reduction in the normalized thickness of stratified flames relative to that of homogeneous flames of the same local equivalence ratio. In addition, they were in agreement with the arguments of Garrido-López and Sarkar [39] and Pasquier et al. [4], in that local differences in $\mathrm{S}_{\mathrm{L}}$ along a stratified flame front (due to regions of different mixture strengths) will effectively cause the front to deform and stretch excessively, acting as an additional mechanism for wrinkling along with turbulence. We observed variations in wrinkling scales along stratified flame fronts in turbulent iso-octane/air V-flames [44], and attributed these variations to local differences in the Lewis number, Le, along the flame front. This lead to a gradual progression from stable fronts with larger smooth curvatures to unstable fronts with elongated structures as the local equivalence ratio progressed from lean to rich.

Variations in curvature were reported by Galizzi and Escudié $[42,43]$ and Dégardin [30], while Galizzi and Escudié [43] observed a bimodal distribution in curvature for stratified cases. Galizzi and Escudié [43] attributed the presence of a secondary mode in the probability density function (PDF) of curvature to separate flame zones, defined at different axial positions in the V-flame, showing that each zone interacted with stratification distinctively. In contrast, Dégardin [30] saw little variation in curvature, where stratified flames showed a more symmetrical distribution with slightly wider range in curvatures than the homogeneous case, similar to the results of Renou et al. [41] for freely-expanding flames. Dégardin [30] also observed that the effects of stratification on curvature were less significant as turbulence intensity was increased, further supporting the experimental results of Samson [49] and numerical predictions of Garrido-López and Sarkar [39]. 
Anselmo-Filho et al. [45] measured the local flame front curvature and surface density along a "stratification surface" of $\phi=0.77$ in turbulent, methane/air V-flames using the Cambridge stratified slot burner. They reported a broader distribution in curvature with a more symmetrical distribution with stratification, in partial agreement with the results of Dégardin [30], while flame surface density consistently increased with stratification. The authors further proposed that the increased surface generation led to a greater overall reaction rate for stratified flames.

Barlow et al. [36] introduced a multicomponent diagnostic in which CO LIF and Raman/Rayleigh scattering were used for instantaneous line measurements of temperature and species concentration through the flame. Their apparatus also allowed for simultaneous measurements of cross-planar OH PLIF to be acquired for 3D topology information. They applied their system to the Cambridge stratified slot burner presented in [45] for locally lean, turbulent, methane/air V-flames and reported instantaneous and mean values of local species concentration and temperature in stratified flames. Their initial results implied that stratification may lead to differences in local flame structure, as they observed gradients in equivalence ratio within the thermal ramp of the reaction zone.

Sweeney et al. [31] continued the work of Barlow et al. [36] on turbulent V-flames with the same burner and diagnostic system. Consistent with the 2D results of Anselmo-Filho et al. [45], they observed a broader distribution in 3D curvature with stratification; however, they also reported a bias shift towards positive curvatures, contrary to Anselmo-Filho et al. [45]. The instantaneous flame thickness, surface density function, scalar dissipation, and flame surface density (FSD) all increased with stratification. They also reported on variations in major species concentrations by presenting data conditioned on the local equivalence ratio, effectively isolating the specific effects of stratification on the local behaviour of flames from the general effects of stratification on the overall behaviour of flame systems. They concluded that the concentrations of major species were reasonably well predicted by laminar flames of the same equivalence ratio, even in stratified flames.

In [47] we proposed a different method of quantifying the specific effects of large-scale, mean gradients in equivalence ratio on the local behaviour of stratified flames. Tracer PLIF of 3-pentanone was used to generate conditioned iso-contours of equivalence ratio up to the mean position of the flame front at 
$\langle c\rangle=0.5$, where $\langle c\rangle$ is the reactant progress variable. Based on the position of the iso-contours, an analysis region of interest (ROI) could then be defined within which the mean range of equivalence ratios was held constant among gradient conditions, effectively isolating the effects of equivalence ratio gradient from those of simple mixture strength. Subsequent $\left(\mathrm{OH}_{\mathrm{LIF}}\right)\left(\mathrm{CH}_{2} \mathrm{O}_{\mathrm{LIF}}\right)$ images within the ROI were then analyzed for variations in local flame surface density and curvature. While small differences in FSD and curvature were observed with gradient, variations were much less significant than those reported in the literature for flame data in which the range of equivalence ratios varied among stratified cases.

Recent experiments on stratified swirl and axisymmetric pilot flame stabilized burners have provided data at high turbulence intensities comparable to those found in practical combustion devices. Bonaldo et al. [35] developed a co-annular weak-swirl burner and conducted experiments on lean methane/air flames. They characterised the 3D flow field with Stereoscopic Particle Image Velocimetry (SPIV). Separate Rayleigh scattering measurements were used to obtain instantaneous temperature data, and tracer PLIF of acetone provided the mean local equivalence ratio. The authors reported broader flame curvature PDFs with stratification, in agreement with [31,41,45], and with a greater probability of positive curvatures, in agreement with [31]. The instantaneous thermal flame thickness decreased with stratification, contrary to the results of Sweeney et al. [31].

The Darmstadt axisymmetric stratified burner was introduced by Seffrin et al. [51] who conducted Laser Doppler Velocimetry (LDV) and high-speed 2D PIV measurements to fully characterize its turbulent flow field. Böhm et al. [46] subsequently characterized the scalar field of the burner with Rayleigh scattering and simultaneous $\mathrm{OH}$ and acetone PLIF. Instantaneous temperature profiles were compared to those of laminar flamelet calculations, with the authors suggesting that local, simultaneous equivalence ratio measurements were required to build statistically viable data conditioned on $\phi$ to quantify the effects of stratification on the local behaviour of flames. Negligible variations in flame front curvature and length were observed with stratification. One dimensional Raman/Rayleigh scattering measurements of local temperature and species concentrations were then performed by Kuenne et al. [52] to validate Large Eddy Simulations (LES). 
Sweeney et al. [32-34] extended their work on turbulent V-flames to the Cambridge turbulent swirl-burner for locally lean methane/air flames using the multicomponent diagnostic system of [36]. They reported results conditioned on the local, instantaneous equivalence ratio for non-swirling [32] and swirling [33] flow conditions. In both cases, stratification had a negligible effect on key combustion species, except for $\mathrm{H}_{2}$ and $\mathrm{CO}$, which increased with stratification when compared to results from laminar premixed flame calculations of the same equivalence ratio. The authors attributed this difference to the direct effect of equivalence ratio gradients. The surface density function and thermal scalar dissipation rate showed negligible effects of stratification. The PDFs of curvature were dominated by turbulence for both nonswirling and swirling cases with little sensitivity to stratification, consistent with [47].

Sweeney et al. [34] further expanded on their previous results on the Cambridge turbulent swirlburner with improved spatial resolution and significantly larger data sets. They reported data conditioned with respect to both the local equivalence ratio and local equivalence ratio gradient. Significant variations in $\mathrm{CO}$ and $\mathrm{H}_{2}$ mass fractions were observed with $\phi$ gradient for lean back-supported stratified flames. Furthermore, both temperature and progress variable gradients were enhanced with stratification, despite negligible changes in flame surface density. Finally, curvature PDFs broadened and shifted towards more negative values with stratification.

Despite the relatively large number of studies on turbulent, stratified flames presented above, most have reported data on the general effects of stratification on the overall behaviour of flame systems. While these studies remain instrumental to characterizing these flames, focus must also be placed on quantifying differences in local flame properties, which are critical to the optimization of numerical models. Conversely, relatively few recent works have attempted to quantify the specific effect of equivalence ratio gradient on local flame properties $[34,47]$ in the context of turbulent flames, and this significant lack of experimental data further confirms the need to extend the currently available database. 
Table 1.2 Summary of literature examining the effects of stratification on flame structure and topology.

$\begin{array}{lll}\text { flame configuration and flow settings approach to stratification anservations and results } & \text { anethodology }\end{array}$

Freely expanding flames

Cho and Santavicca (1993) [16], experimental

- spark ignited stoichiometric $\mathrm{C}_{3} \mathrm{H}_{8} /$ air flame within optically accessible slot plate burner at constant pressure (1 atm) and $300 \mathrm{~K}$

- mean velocity $\overline{\mathrm{U}}=1.16 \mathrm{~m} / \mathrm{s}$

turbulence intensity $22 \%\left(\mathrm{u}^{\prime}=0.25 \mathrm{~m} / \mathrm{s}\right)$

integral length scale $\Lambda \approx 4 \mathrm{~mm}$

- 5 test cases: $100 \%$ premixed ( $0 \%$ relative RMS fluctuation), $75 \%(6 \%), 50 \%(13 \%)$, $25 \%(24 \%), 0 \%(33 \%)$

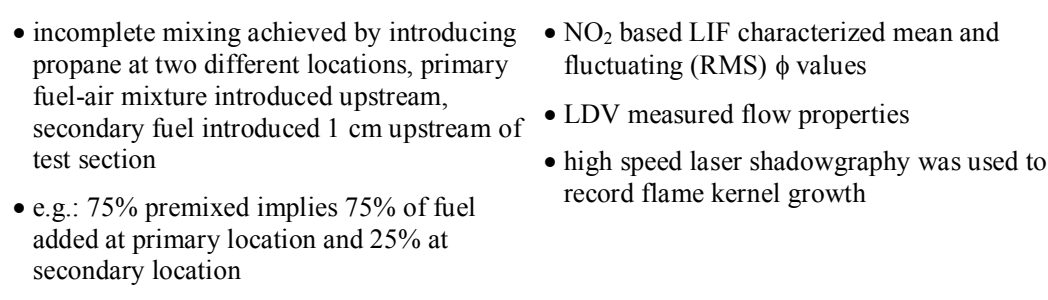

in $\phi$ caused wrinkling and distortion of flame kernel surface

- increasing air/fuel RMS resulted in more wrinkles on flame kernel surface

- "cyclic" variations in flame kernel growth increase as RMS fluctuations in $\phi$ increase

\section{Zhou et al. (1998) [17], experimental}

- spark plug ignited $\mathrm{C}_{3} \mathrm{H}_{8}$ /air flame propagating in cylindrical constant volume combustion chamber with a $80 \mathrm{~mm}$ diameter and $190 \mathrm{~mm}$ length

- turbulence intensity: u' $=0.185 \mathrm{~m} / \mathrm{s}$, $0.130 \mathrm{~m} / \mathrm{s}, 0.100 \mathrm{~m} / \mathrm{s}, 0.0 \mathrm{~m} / \mathrm{s}$

- mean $\phi$ ranging from 0.7 to 1.6
- manual injection of $\mathrm{C}_{3} \mathrm{H}_{8}$ into combustion chamber, degree of fuel-air mixing controlled by moving perforated plates
- Rayleigh scattering for mixture concentration distribution, LDV for turbulence intensity

- Schlieren photography to visualize flame propagation

- topography of flame front was evaluated from wrinkling factor, defined as the ratio of perimeter/projected area of flame front

- flame propagation velocity derived from projected area of flame front
- increased heterogeneity leads to more corrugated flame front compared to homogeneous case of same mean equivalence ratio for both globally lean and rich cases

- flame propagation speed initially increases with degree of heterogeneity before decreasing; observed for globally rich and lean mixtures

- consistent with premixed cases, strong correlation between propagation speed and turbulence intensity in heterogeneous mixture 
Table 1.2 Summary of literature examining the effects of stratification on flame structure and topology. (continued)

observations and results

Samson (2002) [49], experimental

- freely propagating $\mathrm{C}_{3} \mathrm{H}_{8} /$ air flame sparkignited in center of $80 \times 80 \mathrm{~mm}^{2}$ section, $90 \mathrm{~mm}$ behind 6 interchangeable turbulence grids with homogeneous mixture upstream of mixing

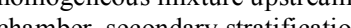
chamber, secondary stratification at base of mixing camber; both upstream of 6 interchangeable turbulence grids

- $\overline{\mathrm{U}} \approx 3.14$ to $4.00 \mathrm{~m} / \mathrm{s}, \mathrm{u}^{\prime} \approx 0.05$ to $0.37 \mathrm{~m} / \mathrm{s}$ $\Lambda \approx 5.0$ to $6.1 \mathrm{~mm}$; isotropic turbulence

- 7 gradient cases

- secondary fuel from nine parallel vertical sections, each with 13 fuel injection ports, generates symmetrical distribution in $\phi$ centered at transverse position $\mathrm{x} \approx 0 \mathrm{~mm}$
- LDV and PIV characterized non reacting flow conditions

- gas analyzer for mixture strength measurement

- simultaneous PIV and acetone tracer PLIF characterized the flame-front structure an local mixture fraction

- $\mathrm{OH}^{*}$ and $\mathrm{CH}^{*}$ chemiluminescence
- optimum level of heterogeneity resulted in greater propagation efficiency

- small-scale variations in mixture strength lead to greater distribution in curvature with length scales on the order of scalar length scales

- large-scale stratification had limited effect on curvature distribution

Renou et al. (2004) [41], experimental

- spark ignited freely propagating $\mathrm{C}_{3} \mathrm{H}_{8} / \mathrm{ai}$ flame as in [49]

- $\overline{\mathrm{U}} \approx 3.14 \mathrm{~m} / \mathrm{s}, \mathrm{u}^{\prime} \approx 0.37 \mathrm{~m} / \mathrm{s}, \Lambda \approx 6.1 \mathrm{~mm}$, $\lambda=2.4 \mathrm{~mm}$; isotropic turbulence

- 3 gradient cases: $\Delta \phi \approx 1.0-0.0$ (mixture fraction gradient 0.0600 )

$\Delta \phi \approx 1.0-0.2$ (mixture fraction gradient 0.0474 )

$\Delta \phi \approx 1.0-0.5$ (mixture fraction gradient 0.0291 )

- 3 homogeneous reference cases:

$\phi \approx 1.1$ (mixture fraction 0.0656 ) $\phi \approx 1$ (mixture fraction 0.0600 )

$\phi \approx 1$ (mixture fraction 0.0600$)$
$\phi \approx 0.9$ (mixture fraction 0.0543 )

- see [49]
- LDV characterized non reacting flow conditions

- simultaneous laser tomography and acetone tracer PLIF characterized the flame-front structure and local mixture fraction

- global flame structure (flame radii, wrinkling ratio, flame speed), local flame structure (2D curvature)
- at early stages of flame propagation, flame structure weakly affected by local heterogeneities

- at higher propagation times, fuel heterogeneities smooth flame front wrinkling

- significant increase in curvature distribution, characteristic length scales of curvature closer to scalar length scales of mixtures strength than integral length scales of turbulence 
Table 1.2 Summary of literature examining the effects of stratification on flame structure and topology. (continued)

\section{Pasquier et al. (2007) [4], experimental}

- freely propagating spark-ignited $\mathrm{C}_{3} \mathrm{H}_{8} /$ air flame propagating from near stoichiometric to lean mixtures

$\bullet \overline{\mathrm{U}} \approx 1.0 \mathrm{~m} / \mathrm{s}, \mathrm{u}^{\prime} \approx 0.3 \mathrm{~m} / \mathrm{s}$

- $60 \times 60 \times 160 \mathrm{~mm}$ constant volume chamber at pressure 1.1 bar

- 3 premixed cases $\phi=1,0.9,0.8$

- 1 stratified case with mean gradient of $\phi=1.0$ to 0.6
- combustion chamber initially filled with $\phi=0.6$ mixture, pure propane injected int mixture at rest

- injection controlled by 2 staggered, solenoid valves which define the degree of solenoid valves
stratification

- large and small-scale stratification analysis methodology observations and results

Rod stabilized V-flames

\section{Dégardin et al. (2006) [38]; Dégardin (2006) [30], experimental}

- rod stabilized (heated rod $0.6 \mathrm{~mm}$ diameter) • see [49] turbulent $\mathrm{CH}_{4} /$ air V-flame

- $\overline{\mathrm{U}}=4.0 \mathrm{~m} / \mathrm{s}, \mathrm{u}^{\prime}=0.06 \mathrm{~m} / \mathrm{s}$

- 6 reference premixed cases from $\phi=1.0$ to

0.5

- 4 stratified cases $\phi_{\text {rod }}=1.2$ to 0.9 with

$\phi_{\min }=0$
- simultaneous PIV and tracer PLIF (methoxybenzene or anisole) for instantaneous velocity and $\phi$

- variation of mean flame radius, from mean cross-sectional area obtained from 50 images

- local burning velocity determined from the local propagation speed of the flame front and the normal component of reactant velocity relative to the flame front
- large-scale stratification lead to an increase in flame propagation

- stratification lead to a greater dispersion in local flame speed as a function of local flame radius

- local distribution in $\phi$ can increase or decrease the local burning velocity 
Table 1.2 Summary of literature examining the effects of stratification on flame structure and topology. (continued)

\section{Robin et al. (2008) [29], experimenta}

- rod stabilized (heated rod), globally lean,

turbulent $\mathrm{CH}_{4} /$ air V-flame

- see [49]

- two turbulence grids $\mathrm{B}$ and $\mathrm{E} ; \overline{\mathrm{U}}=3.75$ and

$3.14 \mathrm{~m} / \mathrm{s}, \mathrm{u}^{\prime} / \overline{\mathrm{U}}=3.7$ and $7.5 \%, \Lambda=5.5$ and

$6.1 \mathrm{~mm}, \lambda=2.9$ and $2.4 \mathrm{~mm}$

- 4 reference premixed flame cases:

grid B: $\phi=0.60,0.70$

grid $\mathrm{E}: \phi=0.55,0.60$

- 5 stratified flame cases:

grid $\mathrm{B}: \phi=0.80-0.00,1.20-0.00$

grid $\mathrm{E}: \phi=0.80-0.00,1.00-0.00,1.20-0.00$ observations and results

\section{Galizzi and Escudié (2006, 2010) [42,43], experimental}

- rod stabilized (2 $\mathrm{mm}$ diameter) globally lean, laminar (2006) and turbulent (2010) $\mathrm{CH}_{4} /$ air V-flame

$$
\text { - } \overline{\mathrm{U}}=5 \mathrm{~m} / \mathrm{s}
$$

laminar case: $\mathrm{u}^{\prime} / \overline{\mathrm{U}}=0.4 \%$

turbulent case: $\mathrm{u}^{\prime} / \overline{\mathrm{U}}=4 \%, \Lambda=5 \mathrm{~mm}$
- two-level fuel injection as in [49]

- primary lean premixed mixture $\phi=0.58$, secondary injection of pure

- normal distribution in $\phi$ centered at transverse position $\mathrm{y}=0=\mathrm{mm}$
- LDV and PIV (solid particulate $\mathrm{ZrO}_{2}$ seed) for turbulent flow properties

- simultaneous acetone tracer PLIF and

Rayleigh scattering for fuel mole fraction and temperature

- characterized local flame structure

(curvature, flame thickness, and fuel mole fraction)

- LW-P numerical model for partially premixed flames [53] based on simplified stoichiometry [54]
- small-scale equivalence ratio fluctuation affect the local flame front causing additional non-negligible stretch, which lead to greater distribution in flame thickness and an overall thinning of the normalized flame thickness

- normalized flame thickness of stratified flame is $10 \%$ thinner than premixed flame at corresponding $\phi$

- LW-P model showed very good agreement with the experimental results
- solid particulate LDA and PIV for in-flame velocity measurements

- laser tomography from incense particle seed

- temperature from thermocouple, $\phi$ from gas analyzer associated to $\mathrm{CH}^{*}$ chemiluminescence
- local increase in laminar flame speed along oblique flame front near stoichiometric region (2006)

- variation in front topology with appearance of "peninsula" along laminar front on the order of stratification zone thickness (2006)

- observed increase in temperature and $\mathrm{CH}^{*}$ fields in the vicinity of "peninsula" (2006)

- stratification caused an expansion of fan angle, increase in flame brush thickness, modification of flow field with product acceleration and deflection (2010) 
Table 1.2 Summary of literature examining the effects of stratification on flame structure and topology. (continued)

\section{Anselmo-Filho et al. (2009) [45], experimental}

- Cambridge stratified slot burner, rodstabilized, globally lean $\phi=0.77$, turbulent $\mathrm{CH}_{4} /$ air V-flame

- $\overline{\mathrm{U}}=3.1 \mathrm{~m} / \mathrm{s}, \mathrm{u}^{\prime}=0.3 \mathrm{~m} / \mathrm{s}, \Lambda=1.9 \mathrm{~mm}$, $\eta=0.09 \mathrm{~mm}$

- 1 reference premixed case $\phi=0.77$

- 5 stratified cases with stratification ratio $\phi_{1} / \phi_{2}=1.0,1.2,1.5,1.8,2.3,3.0$ $\phi_{1}=0.77,0.85,0.92,1.00,1.08,1.16$ $\phi_{2}=0.77,0.69,0.62,0.54$
- slot burner with six 5 x $10 \mathrm{~mm}$ slots

- two pairs of inner channels ( $\phi_{1}$ and $\phi_{2}$ ) controlled independently, two outer slots shielded the flow with air
- single probe hot wire anemometry for flow filed properties

- simultaneous acetone and OH PLIF for fuel concentration and flame front

- quantified variations in turbulent flame brush thickness and incident angle, FSD, and curvature
- stratification increases incident flame angle, flame brush thickness is lowest between $\phi_{1} / \phi_{2}=1.5-2.0$

- FSD increased with stratification with max at $\phi_{1} / \phi_{2}=3.0, \mathrm{FSD}$ profiles skewed towards the products with stratification

- curvature distribution was broader an more symmetrical with stratification compared to premixed flames of same compared to premixed fla
global equivalence ratio

\section{Barlow et al. (2009) [36], experimental}

- Cambridge stratified slot burner as in [45], • see [45]

globally lean $\phi=0.73$

- 1 reference premixed case $\phi=0.73$

- 3 stratified cases with stratification ratio $\phi_{1} / \phi_{2}=1.0,1.86,3.0$ $\phi_{1}=0.73,0.95,1.10$ $\phi_{2}=0.73,0.51,0.3$
- single probe hot wire anemometry for flow field propertie

- simultaneous measurements of spatial distribution of temperature, $\mathrm{CO}_{2}, \mathrm{O}_{2}, \mathrm{CO}$,

$\mathrm{N}_{2}, \mathrm{CH}_{4}, \mathrm{H}_{2} \mathrm{O}, \mathrm{H}_{2}$, for $\phi$ obtained from line imaging of Raman-Rayleigh scattering and two-photon CO-LIF

- simultaneous cross-planar OH-PLIF for instantaneous 3D temperature gradients and geometric information such as turbulent flame brush thickness, flame surface density, and curvature
- introduced diagnostic system capable of simultaneous temperature, major species concentrations, and equivalence ratio

- stratified flames showed significant gradients in equivalence ratio within the instantaneous thermal flame thickness 
Table 1.2 Summary of literature examining the effects of stratification on flame structure and topology. (continued)

observations and results

\section{Vena et al. (2009) [44], experimental}

- NRC stratified slot burner, globally stoichiometric $\phi=1.00$, rod-stabilized, turbulent $\mathrm{C}_{8} \mathrm{H}_{18} /$ air V-flame

- $\overline{\mathrm{U}}=4.6 \mathrm{~m} / \mathrm{s}, \mathrm{u}^{\prime}=0.19-0.33 \mathrm{~m} / \mathrm{s}$

- 1 reference premixed flame setting: $\partial \phi / \partial \mathrm{y}=0.000 \mathrm{~mm}^{-1}$

- 3 gradient flame settings: $\partial \phi / \partial y=0.006,0.019,0.032 \mathrm{~mm}^{-1}$
- $65 \mathrm{~mm}$ x $15 \mathrm{~mm}$ rectangular exit slot burner $\bullet$ single probe hot wire anemometry for cold-

- two fully premixed inlet lines of ( $\phi_{1}$ and $\left.\phi_{2}\right)$

flow turbulence properties

controlled independently, annular air co-

flow

- lower base mixing section of burner subdivide into two triangular sections, proportionate amounts of $\phi_{1}$ and $\phi_{2}$ distributed along transverse axis

- reactants allowed to mix to generate smooth gradients in equivalence ratio at exit of burner 
Table 1.2 Summary of literature examining the effects of stratification on flame structure and topology. (continued)

$\begin{array}{lll}\text { flame configuration and flow settings approach to stratification anservations and results } & \text { analys methodogy }\end{array}$

\begin{tabular}{|c|c|c|c|}
\hline \multicolumn{4}{|l|}{ Sweeney et al. (2011), experimental } \\
\hline $\begin{array}{l}\text { - Cambridge stratified slot burner as in [45], } \\
\text { globally lean } \phi=0.73 \\
\text { - } 1 \text { reference premixed case } \phi=0.73 \\
\text { - } 3 \text { stratified cases with stratification ratio } \\
\phi_{1} / \phi_{2}=1.0,1.86,3.0 \\
\phi_{1}=0.73,0.95,1.10 \\
\phi_{2}=0.73,0.51,0.37\end{array}$ & • see [45] & $\begin{array}{l}\text { - multiscalar diagnostic system from [36] } \\
\text { - presented data conditioned on the local } \\
\text { equivalence ratio }\end{array}$ & $\begin{array}{l}\text { - increases in flame thickness, surface } \\
\text { density function, scalar dissipation, and } \\
\text { FSD in turbulent flames for both premixed } \\
\text { and stratified conditions } \\
\text { - major local species concentrations in } \\
\text { agreement with laminar flames of same } \\
\text { equivalence ratio, even in stratified flames }\end{array}$ \\
\hline \multicolumn{4}{|l|}{ Axisymmetric flames } \\
\hline Bonaldo et al. (2009) [35], experimental & & & \\
\hline $\begin{array}{l}\text { - weakly swirling, globally lean } \phi=0.70 \text {, } \\
0.725 \text {, turbulent } \mathrm{CH}_{4} / \text { air flames } \\
\text { - turbulence parameters in mixing layer: } \\
\overline{\mathrm{U}}=1.51,1.88,2.11 \mathrm{~m} / \mathrm{s}, \mathrm{u} / \mathrm{U}=70,70, \\
80 \%, \Lambda=15 \mathrm{~mm}, \mathrm{~S}=0.09: \\
\text { - } 3 \text { bulk flow cases (velocities of } 6,7,9 \mathrm{~m} / \mathrm{s} \text { ) } \\
\text { - } 6 \text { stratified cases per bulk flow }\end{array}$ & $\begin{array}{l}\text { - exit nozzle with inner (diameter }=35 \mathrm{~mm} \text { ) } \\
\text { and annular (diameter }=65 \mathrm{~mm} \text { ) sections } \\
\text { surrounded by an outer swirling air flow } \\
\text { - equivalence ratios of inner (flow 1) and } \\
\text { annular (flow 2) independently controlled }\end{array}$ & $\begin{array}{l}\text { - stereoscopic planar image velocimetry } \\
\text { (SPIV) at location of flame stabilization, } 3 \\
\text { components of turbulence } \\
\text { - instantaneous temperature fields } \\
\text { characterized from Rayleigh scattering } \\
\text { - average equivalence ratio distributions from } \\
\text { acetone tracer PLIF } \\
\text { - instantaneous burning velocities determined } \\
\text { from SPIV data, flame curvature and } \\
\text { thermal thickness determined from } \\
\text { Rayleigh data }\end{array}$ & $\begin{array}{l}\text { - increased turbulent burning velocity for } \\
\text { steeper } \phi \text { gradients } \\
\text { - reported broader flame curvature PDFs with } \\
\text { stratification with greater probability of } \\
\text { positive curvatures } \\
\text { - instantaneous thermal flame thickness } \\
\text { decreased with stratification }\end{array}$ \\
\hline
\end{tabular}


Table 1.2 Summary of literature examining the effects of stratification on flame structure and topology. (continued)

observations and results

\section{Seffrin et al. (2010) [51], experimental}

- Darmstadt stratified burner, annular exit nozzle, $\mathrm{CH}_{4} /$ air and $\mathrm{C}_{2} \mathrm{H}_{4}$ turbulent flames

- inner slot 1 velocity: $\bar{U}_{1}=10.0,15.0 \mathrm{~m} / \mathrm{s}$ inner slot 2 velocity: $\overline{\mathrm{U}}_{2}=5.0,10.0,15.0$, $20.0 \mathrm{~m} / \mathrm{s}$

- $\mathrm{Re}_{1}=13700,13800,14000,20700$

$\mathrm{Re}_{2}=6600,6700,13300,13500,20000$,

26600

- inner slot 1: $\phi_{1}=0.60,0.90$

inner slot 2: $\phi_{2}=0.60,0.75,0.90$

- 8 reference iso-thermal cases

- 4 reference premixed flame cases

$\phi_{1}=\phi_{2}=0.60,0.90$

- 7 stratified flame cases

$\phi_{1}=0.60,0.90$

$\phi_{2}=0.60,0.75,0.90$
- axisymmetric nozzle with two concentric annular sections (1 and 2) with air co-flow

- inner flows 1 and 2 independently

controlled

- no recirculation, stratified flame stabilized

by inner pilot flame
- Laser Doppler Velocimetry (LDV) and high-speed 2D PIV

Böhm et al. (2011) [46], experimental

- Darmstadt stratified burner as in [51] $\mathrm{CH}_{4} /$ air turbulent flames

- 2 reference premixed flame cases:

$\phi_{1}=0.90, \operatorname{Re}_{1}=13800,13800$

$\phi_{2}=0.90, \mathrm{Re}_{2}=13300,6700$

- 2 stratified flame cases:

$\phi_{1}=0.90, \mathrm{Re}_{1}=13800,13800$

$\phi_{1}=0.90, \mathrm{Re}_{1}=13800,13800$
$\phi_{2}=0.60, \mathrm{Re}_{2}=13300,6700$
- fully characterized turbulent flow field

- significant differences in overall flame

topology among settings, differences more

evident between methane and ethylene fuels
- Rayleigh scattering (temperature) and simultaneous $\mathrm{OH}$ and acetone PLIF
- characterized the scalar field of the burner

- comparisons of instantaneous temperature profiles with laminar flame calculations confirmed the need for simultaneous measurements of local, instantaneous $\phi$ to quantify stratification effects

- negligible variations in flame front curvature and length with stratification 
Table 1.2 Summary of literature examining the effects of stratification on flame structure and topology. (continued)

Kuenne et al. (2012) [52], experimental and numerical

- Darmstadt stratified burner as in [51]

$\mathrm{CH}_{4} /$ air turbulent flames

- 1 reference iso-thermal case:

$\phi_{1}=\phi_{2}=0, \operatorname{Re}_{1}=13800, \operatorname{Re}_{2}=13300$

- 1 stratified flame case:

$\phi_{1}=0.90, \mathrm{Re}_{1}=13800$

$\phi_{2}=0.60, \operatorname{Re}_{2}=13300$
-1D Raman/Rayleigh scattering measurements of local temperature and species concentrations of $\mathrm{CO}_{2}, \mathrm{O}_{2}, \mathrm{~N}_{2}, \mathrm{H}_{2} \mathrm{O}$, and $\mathrm{CH}_{4}$

- 3D LES using 2D tabulated detailed chemistry (CHEM1D and GRI3.0) and thickened flame approach

- computational domain reproduced the geometry of the burner

\section{Sweeney et al. (2012a and 2012b) [32,33], experimental}

- Cambridge stratified swirl burner, annular exit nozzle, globally lean $\phi=0.75, \mathrm{CH}_{4} / \mathrm{ai}$ turbulent flame

- inner annular velocity $\bar{U}_{\mathrm{i}}=8.31 \mathrm{~m} / \mathrm{s}$ outer annular velocity $\bar{U}_{\mathrm{o}}=18.7 \mathrm{~m} / \mathrm{s}$ co-flow air velocity $\overline{\mathrm{U}}_{\text {co-flow }}=0.4 \mathrm{~m} / \mathrm{s}$

- $\mathrm{Re}_{\mathrm{i}}=5960, \mathrm{Re}_{\mathrm{o}}=11500$

$\mathrm{S}=0,0.45,0.79$

- 1 reference premixed case $\left(\phi_{1} / \phi_{2}=1\right)$

$\phi_{1}=\phi_{2}=0.75$

- 2 stratified cases $\left(\phi_{1} / \phi_{2}=2,3\right)$

$\phi_{1}=1.00,1.125$

$\phi_{2}=0.50,0.375$
- annular burner formed of three concentric tubes (radius $=6.35,12.7,19.05 \mathrm{~mm}$ ) surrounded by a circular co-flow (radius $=191 \mathrm{~mm}$ )

- inner tubed caped with a ceramic plug, acts as central bluff body

- inner flow annular flow $\phi_{1}$ in axial direction surrounded by outer annular swirl flow $\phi_{2}$

- burner can be run with or without swirl in outer annular flow $\phi_{2}$
- multiscalar diagnostic system from [36] - presented data conditioned on the local equivalence ratio and
- provided database of species and temperature distribution at 8 axial positions 
Table 1.2 Summary of literature examining the effects of stratification on flame structure and topology. (continued)

flame configuration and flow settings

approach to stratification

analysis methodology

observations and results

Sweeney et al. (2013) [34], experimental

- Cambridge stratified swirl burner as in [32,33],

$\mathrm{CH}_{4} /$ air turbulent flames

- highly swirling flow, $\mathrm{S}=0.79$

- 1 reference premixed case $\left(\phi_{1} / \phi_{2}=1\right)$

$\phi_{1}=0.75, \phi_{2}=0.75$

- 2 stratified cases $\left(\phi_{1} / \phi_{2}=2,3\right)$

$\phi_{1}=1.00,1.125$

$\phi_{2}=0.50,0.375$
- see $[32,33]$

- multiscalar diagnostic system from [36]

- presented data doubly conditioned on the

local equivalence ratio and the local

equivalence ratio gradient
- increases in $\mathrm{CO}$ and $\mathrm{H}_{2}$ in temperature space with lean back-supported stratification

- significant increases in temperature and progress variable gradient within therma ramp with stratification

- 3D flame surface density insensitive to stratification

- broader curvature PDFs with peak shifting towards negative curvatures with stratification 


\subsubsection{Variation in Local and Global Reaction Rate}

Direct experimental measurement of the local combustion intensity along a reacting front has only recently become feasible. While the heat release rate (HRR) may be inferred by indirect flame properties that are physical (flow dilation rate) or chemical (chemiluminscence) in nature, these approaches are not valid in regions of high strain or where local extinctions may occur. Conversely, the approach developed by Paul and Najm [55] in which the product of $\mathrm{OH}$ and $\mathrm{CH}_{2} \mathrm{O}$ concentrations (obtained from Planar Laser Induced

Fluorescence, PLIF) are correlated to the local heat release rate provides direct, spatially and temporally resolved HRR data. This approach has been applied to premixed and diffusion flames in configurations such as laminar counterflow flames [56], turbulent counterflow [57], turbulent bluff body stabilized flames [57,58], turbulent swirl flames [59-61], cone-shaped flames [62], laminar V-flames [63], and lifted jet flames $[64,65]$. Although several experimental studies have inferred differences in reaction rate based on other experimental observables such as flame propagation velocity, flammability limits, or flame surface generation/wrinkling, they did not provide a direct measure of combustion intensity, such that to our knowledge, no experimental HRR data are currently available for turbulent flames in which the $\phi$ gradient is explicitly controlled.

To date, the effects of stratification on HRR have been studied numerically. Direct numerical simulations (DNS) of flame configurations in which an unstrained planar flame is propagated into a stratified mixture have shed light on variations in both the local and global reaction rate in $1 \mathrm{D}[66,67], 2 \mathrm{D}$ [39,66-68], and 3D [66,69]. These studies are detailed in Table 1.3 and further discussed below.

Poinsot et al. [66] conducted 3D DNS of the effect of partial premixing on globally lean $(\phi=0.6)$ propane/air flames at turbulence intensities of $\mathrm{u}^{\prime} / \mathrm{S}_{\mathrm{L}}^{\mathrm{o}}=7.5$ and 2.5 , where $\mathrm{u}^{\prime}$ is the fluctuating component of velocity in the axial $\mathrm{x}$ direction, and $\mathrm{S}_{\mathrm{L}}^{\mathrm{o}}$ is the unstrained laminar flame speed. They reported that local variations in burning rate along the flame front tend to cancel in the mean, resulting in a mass burning rate within $10 \%$ of the reference premixed case. They concluded that differences in global reaction rate are attributable to flame surface generation, for which there was negligible variation between the two turbulence settings. Their results were further supported in 2D DNS of turbulent globally stoichiometric propane/air flames by Haworth et al. [67], who reported small differences in flame length (less than 10\%) 
and global heat release between stratified and premixed flame conditions under comparable turbulence levels of $\mathrm{u}^{\prime} / \mathrm{S}_{\mathrm{L}}^{\mathrm{o}}=4.67$.

Building on the work of Poinsot et al. [66], Hélie and Trouvé [69] evaluated the effect of the length scale $\mathrm{l}_{\phi^{\prime}}$ and scalar distribution $\phi^{\prime}$ of the fluctuations in mixture strength for $\mathrm{u}^{\prime} / \mathrm{S}_{\mathrm{L}}^{\mathrm{o}}=7.5$ in a globally stoichiometric propane/air flame with the same 3D domain as [66]. They observed a decrease in mass burning rate per flame length, in agreement with [66], and concluded that larger fluctuation length scales $l_{\phi^{\prime}}$ decreased the mass burning rate per flame length more significantly, as did larger scalar distributions $\phi^{\prime}$. Contrary to [66], they reported that changes in flame structure did not average out, leading to a decrease in overall mean reaction rate of up to $20 \%$. They also reported negligible stratification induced wrinkling under the turbulent conditions of the study.

Jiménez et al. [68], extended the results of $[66,67,69]$, with 2D DNS of lean propane/air flames, $\phi=0.6$ by evaluating the effect of the length scale $\mathrm{l}_{\phi^{\prime}}$ and both the spatial and scalar distribution $\phi^{\prime}$ of the fluctuations in mixture strength at a high turbulence intensity $\left(\mathrm{u}^{\prime} / \mathrm{S}_{\mathrm{L}}^{\mathrm{o}}=11.6\right)$. They reported an increase in total heat release up to $60 \%$ as the range of $\phi^{\prime}$ broadened within the reactant mixture for length scales $l_{\phi^{\prime}}$ significantly larger than the flame thickness, despite negligible change in total flame length. They also reported an increase in total heat release of up to $50 \%$ with larger stratification length scales $l_{\phi^{\prime}}$, while smaller stratification length scales lead to a reduction in total heat release. The decrease in heat release is consistent with the findings of Hélie and Trouvé [69], who also observed a reduction in total heat release for smaller fluctuation length scales $\mathrm{l}_{\phi^{\prime}}$, which Jimenez et al. argued did not sufficiently wrinkle the flame front to increase total heat release.

The 2D DNS of Garrido-López [39] considered the effects of turbulence for globally lean, $\phi=0.8$ propane/air mixtures, for $\mathrm{u}^{\prime} / \mathrm{S}_{\mathrm{L}}^{0}$ ranging from 0 to 2.65 . They observed an increase in global burning rate for $\mathrm{u}^{\prime} / \mathrm{S}_{\mathrm{L}}^{\mathrm{o}}<1$, consistent with the findings of Jiménez et al. [68], and a decrease in global burning rate for $\mathrm{u}^{\prime} / \mathrm{S}_{\mathrm{L}}^{\mathrm{o}}>1$, as surface generation was not significant enough to counter the decrease in burning rate per unit length, consistent with the findings of [66-69]. The authors also reported that stratification induced surface generation became negligible compared to that induced by turbulence as $\mathrm{u}^{\prime} / \mathrm{S}_{\mathrm{L}}^{\mathrm{o}}$ increased. They proposed that as turbulence intensity increases, the change in local propagation velocity caused by u' becomes 
significantly larger than the induced change in local flame speed $\Delta \mathrm{S}_{\mathrm{L}}$ caused by fluctuations in mixture strength.

DNS of counterflow flames in which the flame front is normal to the gradient vector in equivalence ratio has also been conducted. Marzouk et al. [70] studied the effects of temporal gradients in equivalence ratio in $\mathrm{CH}_{4} /$ air flames. They showed that for timescales lower than $10 \mathrm{msec}$, variations in equivalence ratio broadened the reaction zone, enhanced the burning rate, and extended the lower flammability limit, similar to the effects of larger-scale gradients reported by Ra and Cheng [71], Kang and Kyritsis [22-25], and Pires Da Cruz [26] for freely expanding and pipe-flow type flames. They concluded that gradients in equivalence ratio with time/length scales on the same order as those of the flame provide back support for a flame due to higher temperature and radical concentrations in the flame's wake, which serve as a "heat (and chemical) reservoir" for upstream reactants.

Richardson et al. (2010) studied the effects of stratification on both steady ( $\phi$ gradient remained constant) and un-steady ( $\phi$ gradient varied in time) lean $\mathrm{CH}_{4} /$ air flames in a counterflow configuration. They observed significant differences in $\mathrm{H}, \mathrm{H}_{2}$, and $\mathrm{OH}$ concentration with $\phi$ gradient, with greater upstream fluxes of these species in back supported flames, and concluded that the modification of HRR in lean flames is dominated by species fluxes, contrary to the observations of Kang and Kyritsis [23]. An increase in peak HRR and propagation velocity, as well as a decrease in HRR profile thickness were also observed in back supported flames, while the opposite behaviour was observed in front supported flames. Temporal variations in $\phi$ lead to a small time delay between $\phi$ and flame speed. Reported trends were consistent with those observed in steady flames as the propagation velocity of unsteady flames increased with back-support and decreased with front-support. The authors concluded that, in addition to the local equivalence ratio, the local propagation speed of stratified flames also depends on the $\phi$ gradient as well as unsteady terms caused by the strain rate and $\phi$ fluctuations.

Zhou and Hochgreb (2013) conducted DNS of $\mathrm{CH}_{4} /$ air flames in a counterflow configuration in which a reactant stream of equivalence ratio $\phi_{\mathrm{R}}$ is opposed to a hot equilibrium stream $\phi_{\mathrm{P}}$. They studied the effects of front and back support on the HRR at different local stoichiometries and $\phi$ gradients, and observed a monotonic increase in HRR relative to premixed reference flames for all back supported flames, 
and a decrease in HRR for all front supported flames. Extensions of upper and lower flammability limits were also observed. Results indicated that different mechanisms of back support occurred for lean (heat) and rich (species, $\mathrm{H}_{2}$ ) flames, contrary to the findings of Richardson et al [72], yet consistent with Kang and Kyritsis [22-25], and Pires Da Cruz [26]. 
Table 1.3 Summary of literature examining the effects of stratification on both the local and global reaction rate.

observations and results

Unstrained premixed flame propagating into stratified mixture

Poinsot et al. (1996) [66], numerical

- 1D, 2D, and 3D DNS of globally stoichiometric $\phi=1.0$, laminar and turbulent $\mathrm{C}_{3} \mathrm{H}_{8} /$ air flames

- planar premixed flame propagating into mixture of spatially varying equivale ratio (small-scale perturbations)

reactants on left side of domain $\left(\mathrm{x}_{1}<0\right)$ products on right $\left(\mathrm{x}_{1}>0\right)$

- $\mathrm{u}^{\prime} / \mathrm{S}_{\mathrm{L}}=7.5$ and 2.5

- case 1: 1D unsteady laminar, stratification in $\mathrm{x}$, direction of flame propagation

- case 2: 2D steady, stratification in y, transverse to flame front

- case 3: 2D unsteady, stratification in $\mathrm{x}$ and

- case 4: 3D turbulent flame, random mixture distribution, single step $\mathrm{C}_{3} \mathrm{H}_{8}$ reaction mechanism
- reactant mixture defined in terms of probability density function, integral length scale, and spatial distribution of inhomogeneity (can be in $x, y, x y$, and $x y z$ directions)

- local mixture fraction defined in terms of mean and varying components (gradients for laminar cases, fluctuations (RMS) for turbulent cases)

- spatial differentiation accurate to the sixorder [73], temporal differentiation from $3^{\text {td }}$ order Runge-Kutta, Navier Stokes

characteristic boundary condition procedure [74]

- effects on local flame structure evaluated from mass burning rates

- effects on global flame behaviour evaluated from total reaction rate ratios of partially premixed and premixed cases
- significant variations in flame structure were observed in terms of mass burning rate per unit flame surface area

- if no quenching is observed, variations on flamelet structure average out, resulting in limited partial premixing effects on flame structure

- strong variations in mixture composition (large amplitudes and length scales) lead to quenching

- increase in flame stretch was observed in the absence of quenching with enhanced wrinkling and a faster burning rate

- in highly turbulent cases, turbulence induced wrinkling is dominant over partial premixing induced wrinkling (which has a negligible affect) 
Table 1.3 Summary of literature examining the effects of stratification on both the local and global reaction rate. (continued)

observations and results

\section{Hélie and Trouvé (1998) [69], numerical}

-3D DNS of globally stoichiometric $\phi=1.0$, $\bullet$ see [66] planar laminar $\mathrm{C}_{3} \mathrm{H}_{8} /$ air flames

- decaying isotropic turbulent flow,

$\mathrm{u}^{\prime} / \mathrm{S}_{\mathrm{L}}=7.5$

- reactants on left side of domain $\left(x_{1}<0\right)$

products on right $\left(\mathrm{x}_{1}>0\right)$
- RMS fluctuations $\phi^{\prime}=0.3,0.6$

- Length-scales of fluctuations on order of

integral length scale $1_{t}$, such that $l_{\mathrm{z}} / \Lambda=1,2$
- see [66]

- single-step chemistry and unity Lewis

number

- evaluated variations in flame topology an turbulent reaction rate

Haworth et al. (2000) [67], numerica

- 1D laminar and 2D DNS of globally

stoichiometric $\phi=1.0$, turbulent $\mathrm{C}_{3} \mathrm{H}_{8} /$ air

flames

- reactant conditions similar to those found in

an automotive engines

- $\mathrm{u}^{\prime} / \mathrm{S}_{\mathrm{L}}=4.67$

- pressure $=4 \mathrm{~atm}$, temperature $=700 \mathrm{~K}$

- 1 reference premixed case $\phi=1.0$

- 1 stratified case, $\phi_{\min } / \phi_{\max }=0.5 / 2.7$
-1D: step change in $\phi$ from $\phi=1.0-0.5$ and $\bullet$ see $[66,69]$

step change in $\phi$ from $\phi=1.0-2.0$

- Sinusoidal variation in $\phi$
- detailed chemical kinetics, 29 species, 73 reaction mechanism [75]

- $2 \mathrm{~mm}$ x $2 \mathrm{~mm}$ computational domain
- negligible variations in flame stretch and surface production with partial premixing

- significant differences in flamelet structure and mass burning rate per unit flamesurface area

- $20 \%$ reduction in global reaction rate with partial premixing 
Table 1.3 Summary of literature examining the effects of stratification on both the local and global reaction rate. (continued)

observations and results

\section{Jiménez et al. (2002) [68], numerical}

-2D DNS of globally lean $\mathrm{C}_{3} \mathrm{H}_{8} /$ air flame

$\bullet \mathrm{u}^{\prime} / \mathrm{S}_{\mathrm{L}}=11.6$

- 1 reference premixed case $\phi=0.6$

- 3 stratified cases, globally lean $\phi=0.6$ $\phi_{\min } / \phi_{\max }=0.06 / 1.14, \phi^{2}=0.15,1_{\phi} / \mathrm{L}_{\mathrm{y}}=1$ $\phi_{\min } / \phi_{\max }=0.03 / 2.06, \phi^{, 2}=0.45,1_{\phi} / \mathrm{L}_{\mathrm{y}}=1$ $\phi_{\min } / \phi_{\max }=0.03 / 2.06, \phi^{, 2}=0.45,1_{\phi} / \mathrm{L}_{\mathrm{y}}=1 / 4$
- initially lean $(\phi=0.6)$ laminar flame propagating into turbulent stratified mixtures with $\phi_{\min }=0.03-0.06$ and $\phi_{\max }=1.14-2.06$

- $\phi$ distribution varies transversely relative to initially planar flame front
- see $[66,67,69]$

- detailed chemical kinetics, 30 species, 76 reaction mechanism

- significant difference in heat release between premixed and partially premixed flames

- higher mean heat release rate per unit flame length resulted in efficiency increase up to $60 \%$ given the appropriate PDF of mixture $60 \%$ given
distribution

- spatial distribution of heterogeneities affects wrinkling of flame front

- variations in length scales, the size of the rich/lean pockets, resulted in variations in efficiency of approx. 50\%

- reported secondary reaction zone in postflame region

Garrido-López and Sarkar (2005) [39], numerical

- 2D DNS of globally lean $\phi=0.8$ fueloxidizer mixture with reactant propertie based on those of $\mathrm{C}_{3} \mathrm{H}_{8} /$ air mixtures

- velocity and compositional fluctuations larger than characteristic flame thickness

- $\mathrm{u}^{\prime} / \mathrm{S}_{\mathrm{L}}^{\mathrm{o}}=0-2.65$

- 4 reference premixed cases

- 12 stratified cases
- reference planar lean laminar flame propagating towards reactants of varying mixture composition
- one-step chemical model, unity Lewis number for all species

- time advancement performed with $3^{\text {rd }}$ order Runge-Kutta scheme
- mixture inhomogeneity resulted in significant increase in flame length for small velocity fluctuations $\left(\mathrm{u}^{\prime} / \mathrm{S}_{\mathrm{L}}<1\right)$ and weaker increase for greater velocity fluctuations

- reduced burning rate per unit length of flame front observed with increased levels of inhomogeneity and globally leaner mixtures

- increase in rms of mixture fraction fluctuations caused an increase in overall burning rate for $\mathrm{u}^{\prime} / \mathrm{S}_{\mathrm{L}}<1$ and a decrease for more intense velocity fluctuations 
Table 1.3 Summary of literature examining the effects of stratification on both the local and global reaction rate. (continued)

$\begin{array}{lll}\text { flame configuration and flow settings approach to stratification abservations and results } & \text { analys methodogy }\end{array}$

Stagnation type flames

Marzouk et al. (2000) [70], numerical

- unsteady strained premixed lean $\mathrm{CH}_{4} / \mathrm{air}$

flame with an axisymmetric stagnation-

point flow

- reactants at $300 \mathrm{~K}$ with products under

adiabatic and constant pressure

- small-scale temporal $\phi$ gradients from

stoichiometric to lean mixtures

- equivalence ratio length and timescales

comparable to those of the flame
-1D unsteady strained code based on globalized Newton iterative method an preconditioned Krylov subspace linea solver

- $\mathrm{C}(1)$ kinetic model for $\mathrm{CH}_{4} /$ air combustion [76], 46 reactions, 16 species
- timescales lower than $10 \mathrm{~ms}$ broadened the reaction zone, enhanced the burning rate, and extended the lower flammability limit

- back support due to higher temperature and radical concentrations in the flame's wake, which serve as a "heat (and chemical) reservoir" for upstream reactants

Richardson et al. (2010) [72], numerical

- 1D laminar axisymmetric counterflow $\mathrm{CH}_{4} /$ air flames

- 5 steady flame configurations:

1 reference premixed case: $\phi=0.75$

$\overline{\mathrm{U}}=0.6 \mathrm{~m} / \mathrm{s}$

2 front supported cases: $\phi=0.9-0.6$

$\overline{\mathrm{U}}=0.6,1.8 \mathrm{~m} / \mathrm{s}$

2 back supported cases: $\phi=0.6-0.9$

$\overline{\mathrm{U}}=0.6,1.8 \mathrm{~m} / \mathrm{s}$

- 4 unsteady flame configurations:

$\phi^{\mathrm{o}}=\phi^{\mathrm{m}}+\mathrm{A} \sin (2 \pi \mathrm{ft})$

$\phi^{\mathrm{m}}=0.75$

$\mathrm{A}=0.28,0.34,0.48,0.90$

$\mathrm{f}=10,50,100,200 \mathrm{~Hz}$

strain rate $=300 \mathrm{~s}^{-1}$

$\overline{\mathrm{U}}=0.6 \mathrm{~m} / \mathrm{s}$
- reactant-to-product configuration with steady flame stabilized in mixing layer for spatial $\phi$ gradients

- reactant-to-reactant configuration with twin-flames exposed to time-varying $\phi$ oscillations

- 1D deta

- steady simulations: OPPDIFTM [77]

- unsteady simulations: OPUS [78]

- reaction rates and thermodynamic and transport properties: CHEMKINTM [79]

- $\mathrm{CH}_{4} /$ air chemistry: 53 species GRI 3.0 natural gas mechanism
- significant differences in $\mathrm{H}, \mathrm{H}_{2}$, and $\mathrm{OH}$ concentration with $\phi$ gradient, with greater upstream fluxes of these species in back supported flames, leading to enhanced HRR

- increase in peak HRR and propagation velocity, decrease HRR profile thickness in back supported flames, opposite behaviour observed in front supported flames

- temporal variations in $\phi$ lead to a small time delay between $\phi$ and flame speed

- local propagation speed of stratified flames depends on $\phi$ gradient and unsteady terms caused by strain rate and $\phi$ fluctuations 
Table 1.3 Summary of literature examining the effects of stratification on both the local and global reaction rate. (continued)

\section{Zhou and Hochgreb (2013) [80], numerical}

-1D laminar axisymmetric counterflow $\mathrm{CH}_{4} /$ air flames

- 4 steady flame configurations:

2 reference premixed cases: $\phi=0.6,0.9$

1 back supported case: $\phi=0.6-0.9$

1 front supported case: $\phi=0.9-0.6$

strain rate $=50 \mathrm{~s}$

- 4 steady flame configurations for

flammability limit:

2 back supported cases:

$\phi=0.10-0.50, \phi=1.90-2.15$

2 front supported cases:

$\phi=0.50-0.35, \phi=2.10-1.90$

strain rate $=50 \mathrm{~s}^{-1}$

- unsteady flame configuration:

$\phi_{\mathrm{R}}=\phi_{\mathrm{o}}+\mathrm{A}_{\mathrm{o}} \sin (2 \pi \mathrm{ft})$

$\phi_{\mathrm{o}}=0.50, \mathrm{~A}_{\mathrm{o}}=0.50, \mathrm{f}=50 \mathrm{~Hz}$

$\overline{\mathrm{U}}=0.2 \mathrm{~m} / \mathrm{s}$

strain rate $=50 \mathrm{~s}^{-1}$
- reactant to product configuration with

steady flame stabilized in spatial $\phi$ gradien

- unsteady flame in sinusoidal evolution of

temporal $\phi$ fluctuations
- 1D detailed $\mathrm{CH}_{4} /$ air chemistry and transport model

- steady and unsteady simulations done in CORSILAB

- $\mathrm{CH}_{4} /$ air chemistry: 53 species GRI 3.0 natural gas mechanism
- monotonic increase in HRR relative to premixed reference flames for back

supported flames, decrease in HRR for front supported flames

- extensions of upper and lower flammability limits were observed with stratification

- unsteady and steady flames showed similar variations with $\nabla \phi$

- HRR primarily a function of local $\phi$ and

$\nabla \phi$, unsteady effects unnecessary to explain effect of stratification

- different mechanisms of back support

occurred for lean (heat) and rich (species)

flames 


\subsection{Thesis Motivation and Problem Definition}

Evidence of the interaction between neighbouring flame regions, or flamelets, has shown that diffusive fluxes of heat/mass from downstream heated products may back support the leading reaction zone and alter combustion, such that the local behaviour of flames cannot be predicted solely based on initial reactant properties. For laminar flame configurations in which the flame front propagates solely in the direction of the gradient vector in equivalence ratio, differences in flammability limits and local flame propagation velocity have been observed experimentally in freely propagating and pipe-flow type flames, while DNS of 1D counterflow flames have reported differences in local heat release rate. Quantifying these flamelet interactions within turbulent flames is inherently more complex, as the general effects of stratification on the global behaviour of a flame system must be decoupled from those of specific $\phi$ gradient on local flame properties. This complexity necessitates the development of a well-defined experimental methodology, combined with a non-intrusive multicomponent diagnostic system, such that little experimental data are currently available.

The primary motivation of this thesis was to address this lack of experimental data and develop a novel analysis approach to clearly define the experimental question when attempting to discern the specific effects of $\phi$ gradient on combustion. The main objectives of this work were to: 1) commission and characterize a burner capable of accurately and precisely reproducing mean gradients in equivalence ratio in a turbulent flow field, 2) set up a laser based diagnostic system and develop a novel experimental methodology to evaluate the local dynamic properties along a stratified flame front, and 3) quantify the effects of mean gradients in equivalence ratio on local flame behaviour in terms of flame topology and heat release rate.

\subsection{Overview of Thesis}

The thesis follows the research chronologically. Preliminary work was done to characterize the general structure of the turbulent, stratified V-flame as it was stabilized in reactant mixtures with different mean gradients in equivalence ratio. Measurements of chemiluminescence imaging, spectroscopy, and $\mathrm{OH}$ 
PLIF were done and these initial results published as a Society of Automotive Engineers (SAE) conference paper [44]. This article is not included in the thesis.

Focus was then placed on devising a methodology to evaluate the specific effects of mean gradients in equivalence ratio on local flame properties, separate from those attributable to simple variations in mixture strength. This was accomplished with a unique, windowing technique, in which the width of the analysis region of interest (ROI) was adjusted based on the imposed equivalence ratio gradient at the exit of the burner. This ensured fair comparison among gradient conditions as the mean range of equivalence ratios being analysed was kept constant among flame settings. Specifics on the windowing approach are presented in a first article (Chapter 2), "Equivalence ratio gradient effects on flame front topology in a stratified iso-octane/air turbulent V-flame" (published in the Proceedings of the Combustion Institute), where details of the image processing routines are outlined. The experimental apparatus is introduced and the Planar Laser Induced Fluorescence (PLIF) diagnostic is presented. At the time of publication, the data presented were the first reported specific effects of mean equivalence ratio gradients in turbulent flames. Results showed that mean $\phi$ gradients for near-stoichiometric flames led to smaller variations in flame topology (in the context of local FSD and curvature PDFs) than those attributed to the general effects of stratification.

The second article, "Heat release rate variations in a globally stoichiometric, stratified isooctane/air turbulent V-flame" (submitted to Combustion and Flame) is presented in Chapter 3, and builds on the flame topology results of the first article. The ROI approach was utilized to investigate comparative heat release rates (determined from the product of $\left(\mathrm{OH}_{\mathrm{LIF}}\right)\left(\mathrm{CH}_{2} \mathrm{O}_{\mathrm{LIF}}\right)$ ) at different mean equivalence ratio gradients. These are the first such measurements in turbulent iso-octane/air flames. Detailed numerical simulations of $1 \mathrm{D}$ laminar iso-octane/air premixed flames were conducted to validate the $\left(\mathrm{OH}_{\mathrm{LIF}}\right)\left(\mathrm{CH}_{2} \mathrm{O}_{\mathrm{LIF}}\right)$ approach for HRR measurements in iso-octane/air flames over a broad range of equivalence ratios. Based on the results of the laminar flame simulations, potential temperature effects on the $\mathrm{OH}$ and $\mathrm{CH}_{2} \mathrm{O}$ fluorescence signals within each ROI were determined (calculated from Boltzmann population fractions and collisional quenching rates of both species over the range of equivalence ratios specific to each ROI). A Monte Carlo (MC) analysis was presented that further enables direct interpretation of mean $\phi$ gradient effects on the mean HRR of near-stoichiometric flame regions. Variations in mixture strength within each 
ROI were calculated (independent of shot noise), which could be attributed to turbulent mixing of the imposed $\phi$ gradient at the exit of the burner. This enabled assessment of the resulting temperature effects on the $\mathrm{OH}$ and $\mathrm{CH}_{2} \mathrm{O}$ fluorescence signals. Results demonstrated that the combined effect of temperature variations and the broader range of $\phi$ within the ROI, would lead to an overall net decrease in mean HRR within the ROI less than $1.0 \%, 1.6 \%, 2.7 \%$, and $4.3 \%$ for the weakest to steepest gradient cases respectively. By contrast, measured decreases in HRR directly attributable to mean $\phi$ gradient effects exceeded this range by a factor of $\sim 2$ to 3 . These results were in line with 1D DNS of counterflow flames, such that the HRR data showed evidence of thermal back support from the heated products within the Vflame. General stratification effects were separately investigated by comparing gradient cases with relatively constant mean global equivalence ratios and broader ranges of mixture strengths. Results highlighted the competing mechanisms of increased flame surface generation and decreased HRR per unit flame length as they defined the overall HRR of the flame system. This analysis further confirmed that the effects of mean $\phi$ gradient on local flame properties, and the general stratification effects on global flame behaviour differ, further emphasizing the importance of proper interpretation of data.

The third article, "A comparative analysis on the effects of stratification on locally lean, nearstoichiometric, and rich iso-octane/air turbulent V-flames" (to be submitted to Combustion and Flame), extends the analysis conducted in the first two papers and compares the effects of large-scale mean gradients in equivalence ratio in locally rich, near-stoichiometric, and lean flame regions. Instantaneous $\left(\mathrm{OH}_{\mathrm{LIF}}\right)\left(\mathrm{CH}_{2} \mathrm{O}_{\mathrm{LIF}}\right) \mathrm{HRR}$ images were analysed for variations in local peak HRR and instantaneous flame thickness $\delta_{\mathrm{t}}$ among $\phi$ gradient conditions within each ROI.

A Monte Carlo analysis was used to estimate total experimental uncertainties in the measurement of local curvature, and relative uncertainties in the measurement of peak HRR and flame thickness. Differences among flames conditions were first quantified by plotting normalized distributions of local peak HRR and $\delta_{\mathrm{t}}$ with local curvature $\kappa$. Results for the three fully premixed reference cases showed general variation of peak HRR with curvature that reflected the influence of competing effects of mass and thermal diffusion, in line with that expected based on thermodiffusive theory $[19,81]$. The normalized 
distributions of $\delta_{\mathrm{t}}$ with local curvature for the premixed cases were also consistent with the literature [29], and were insensitive to mixture stoichiometry.

When comparing the distributions of peak HRR with curvature among gradient conditions, subtle variations were observed. The data provided evidence of the potential interaction between neighbouring flame regions, and further suggested that stratification may influence the local thermodiffusive stability of the wrinkled structures along the turbulence flame front.

The effects of mean $\phi$ gradients on the ensemble average of peak HRR and $\delta_{t}$ were separately evaluated following the same MC approach described in Chapter 3, for article 2. Small but repeatable differences were observed among gradient flame configurations, as results showed evidence of interaction between the heated products and the leading stratified branches, as front- and back-supported flames showed opposite trends. Locally rich, back-supported flame regions showed a gradual increase in peak HRR of 9.9-10.2\% (depending on the $\mathrm{CH}_{2} \mathrm{O}$ quenching model employed) which was accompanied by a decrease in instantaneous flame thickness $\delta_{\mathrm{t}}$ of $7.1-7.2 \%$ as the gradient steepened. Front-supported rich flames showed the opposite behaviour, as the peak HRR decreased by $5.1-5.3 \%$, and $\delta_{\mathrm{t}}$ increased by $2.3-$ 2.4\%. Similar but weaker trends were observed in near-stoichiometric flame regions, as the steepest gradient case led to a decrease of $3.3-3.5 \%$ in peak HRR and an increase of $2.7-2.8 \%$ in $\delta_{t}$. Locally lean flame regions showed a small increase in peak HRR with back-support of 3.8\%, and a decrease in $\delta_{\mathrm{t}}$ of 2.02.1\%. While trends for back-supported lean flames were generally analogous to those of back-supported rich flames, they were consistently less pronounced for equivalent gradient levels. These results suggest that back-support may be less important for flames nearer to stoichiometric, and further imply that the dominant mechanisms of back-support may differ for rich and lean flames.

These three articles demonstrate the achievement of the goals of this thesis: to develop an experimental apparatus and methodology that quantifies the effects of mean gradients in equivalence ratio on the local topology and heat release rate of stratified iso-octane/air turbulent flames; to provide evidence of the potential interaction between neighbouring flame regions; and to gain insight into the physical mechanisms that alter the local behaviour of partially premixed flames. 


\section{Chapter 2}

\section{Equivalence Ratio Gradient Effects on Flame Front Topology in a Stratified Iso-Octane/Air Turbulent V- Flame}

This chapter has been peer-reviewed and was published in the Proceedings of the Combustion Institute in January 2011 (first available online on August 6, 2010). It should be cited as:

Vena, P.C., Deschamps B., Smallwood, G.J., Johnson, M.R. (2011) "Equivalence ratio gradient effects on flame front topology in a stratified iso-octane/air turbulent V-flame", Proceedings of the Combustion Institute, 33:1551:1558. DOI: 10.1016/j.proci.2010.06.041.

This paper was co-authored by the thesis author, Patrizio Vena, Dr. Béatrice Deschamps, Dr. Greg Smallwood, and Prof. Matthew Johnson. Mr. Vena set up, tested, and characterized the measurement equipment; performed the measurements, uncertainty analysis, and initial data analysis; and wrote a first draft of the manuscript. Dr. Smallwood participated in the initial design of the burner, provided access to laboratory facilities, and shared in funding the project. The theory development, final data analysis, and editing and revision of the manuscript were conducted jointly by Mr. Vena, Dr. Deschamps, and Prof. Johnson.

\subsection{Abstract}

The effect of partial premixing on the local topology of globally stoichiometric flames was considered using a novel burner that permits controlled transverse variation of equivalence ratio along a continuous stratified flame front. Five iso-octane/air V-flames with different levels of stratification were studied in experiments designed to quantify differences in flame surface density and curvature between a reference homogeneous case and four different gradient cases. The mean equivalence ratio along the stratified flame front was characterized for each flame condition using 3-pentanone planar laser induced fluorescence (PLIF) conditioned to the reactants. PLIF of $\mathrm{OH}$ and $\mathrm{CH}_{2} \mathrm{O}$ were subsequently used to obtain progress variable, flame surface density, and curvature measurements for locally stoichiometric flamelets subjected to varying equivalence ratio gradients. To ensure fair comparison among conditions and to eliminate potential effects due to variable mixture strengths, a unique variable interrogation window approach was 
used to fix the range of equivalence ratios considered in the analysis of flames under different gradients. Results showed a significant increase in flame wrinkling with increased gradients in equivalence ratio, as well as increased variability in the local intensities of OH PLIF images. The effect of increased flame surface density was more modest, balanced in part by an increase in flame brush thickness and ultimately by a decrease in flame length. Observed variations in curvature distribution were not nearly as prominent as those reported in the literature for globally lean methane/air V-flames, which suggests that although gradients in mixture strength may alter the overall structure and instantaneous behaviour of globally stoichiometric combustion systems, their effect on the topology of locally stoichiometric flames may be limited. 


\subsection{Introduction}

Partial premixing is an indispensable tool in the optimization of practical combustion devices such as stratified charge engines and gas turbines. Studies have confirmed potential gains in overall thermodynamic efficiency via reductions in fuel consumption $[8,11,12]$ and pollutant emissions $[8,10,11]$, highlighting the need for greater understanding of the physical mechanisms responsible. However, because of the inherent complexity of turbulent, partially premixed flames, the development of a well defined methodology to quantify the effects of stratification is a significant experimental challenge. If accurate physical models are to be developed, an experimental database is necessary to validate the results obtained through numerical predictions [45].

Several researchers have considered the effects of stratification on flames including the effects of small-scale variations in mixture strength defined in terms of local inhomogeneities [16,17], or of largerscale variations associated with gradients in equivalence ratio $[4,21,22,24-26,45,70]$. In addition, works by Renou et al. [41], Robin et al. [29], and Garido-López and Sarkar [39] have attempted to define how these effects may be coupled to those of turbulence. Despite differing results in some of these studies, there is cause to suggest that adjacent flamelets of different mixture strengths can interact, potentially altering their expected behaviour predicted solely from reactant properties. Robin et al. [29] suggest that the three most notable effects of stratification are (1) the extension of the lower flammability limit, (2) the change in flame structure and topology, and (3) the variation in reaction rate.

Several recent studies have been performed on freely propagating flames, where the flame propagates solely in the direction of the gradient vector in mixture fraction, through large-scale variations in equivalence ratio $[21,22,24-26,70]$. Under these conditions, a flux of heat and radicals from the burnt richer region feeds the leaner region, and enhances its resistance to extinction. This is often referred to as "back support" or a "memory effect" in that the laminar flame speed $\left(\mathrm{S}_{\mathrm{L}}\right)$ and reaction rate are not determined strictly by the reactant properties, but also by the composition and temperature of the burnt gas.

Variations in flame topology with partial premixing have also been observed in terms of flame thickness and rate of strain [29,30], flame length and wrinkling scales [16,17,29,30,39,41,44,45,50], flame surface density [45], and curvature [30,39,41,45,50]. Experiments on freely propagating flames by Zhou et 
al. [17] and Cho and Santaviccia [16] suggested that small-scale stratification generally enhances flame wrinkling, resulting in a more corrugated front with increased flame surface area relative to the homogeneous case. Local differences in $\mathrm{S}_{\mathrm{L}}$ along a stratified flame front, due to regions of different mixture strengths, will cause the front to deform and stretch excessively, acting as an additional mechanism for wrinkling along with turbulence [29].

Experiments on rod-stabilized V-flames $[29,30,44,50]$ have shown a general increase in wrinkling for flames stabilized in stronger transverse gradients in equivalence ratio. Variations in curvature were reported by Galizzi [50] and Dégardin [30], while Galizzi [50] observed a bimodal distribution in curvature for stratified cases. Galizzi [50] attributed the presence of a secondary mode in the probability density function (PDF) of curvature to separate flame zones, defined at different axial positions in the V-flame, showing that each interacted with stratification distinctively. Vena et al. [44] also identified flame zones within partially-premixed iso-octane/air V-flames and argued that the local variation in the Lewis number along the flame front leads to a progression from stable fronts with larger smooth curvatures to unstable fronts with elongated structures.

Anselmo-Filho et al. [45] measured the local flame front curvature and surface density along a "stratification surface" of $\phi=0.73$ for a turbulent methane/air V-flame, reporting variations in both curvature and flame surface density as stratification increased. They proposed that the increased surface generation was a contributing mechanism to the greater levels of heat release often reported for stratified mixtures. Although some DNS simulations have predicted either a negligible variation $[66,67]$ or even a reduction [69] in heat release, it is generally agreed that partial premixing leads to increases in overall reaction rate. Experimental results of Zhou et al. [17] have shown that the degree of heterogeneity significantly increases flame propagation speed up to an "optimal" level of stratification and suggest that greater levels of heat release may result with small-scale stratification. The 2D DNS of Jiménez et al. [68] on iso-octane/air flames supported the results of Zhou et al. [17] and further concluded that both the length scales and spatial distribution of heterogeneities may lead to increases in heat release of up to $60 \%$.

Much of the difficulty in understanding the effects of partial premixing arises because it is generally quite challenging experimentally to separate potential effects of stratification from simple 
mixture strength effects. In the present work, a unique approach is used to investigate topology of turbulent iso-octane/air V-flames over a range in equivalence ratios that is constant among flame conditions, allowing specific effects of large-scale equivalence ratio gradients to be evaluated. A novel, variable stratification burner was employed that enables transverse variation of equivalence ratio along a flame front. Coupled with dual-wavelength PLIF to simultaneously image of $\mathrm{OH}$ and $\mathrm{CH}_{2} \mathrm{O}$, experiments were performed to characterize gradient effects on flame front topology in terms of flame brush thickness and incident angle, and to quantify the effects on flame surface density and curvature. Through careful analysis of the results using an equivalent windowing technique to control and fix the range of mixture strengths for different gradient conditions, the effects of large-scale equivalence ratio gradients on local front behaviour are described.

\subsection{Experimental Setup}

A $63 \mathrm{~mm} \times 15 \mathrm{~mm}$ rectangular exit burner was designed that permits controlled variation of the local equivalence ratio along the y-axis (transverse axis) as shown in Figure 2.1. Two fully premixed iso-octane (i.e. 2,2,4-trimethylpentane)/air reactant streams of different mixture strengths $\phi_{1}$ and $\phi_{2}$ enter separate compartments within the $16 \mathrm{~mm}$ high lower section of the burner (section 1). The two streams pass through a $15 \mathrm{~mm}$ wide by $65 \mathrm{~mm}$ long by $12.7 \mathrm{~mm}$ thick section of expanded metal foam (shown as the orange block in Figure 2.1) which ensures the flow is uniformly distributed as it enters the lateral mixing section $(2 \mathrm{a}, \mathrm{b})$ of the burner. A sealed barrier cuts diagonally across the metal foam and partitions the flow into two equal sized triangular sections. Proportionate amounts streams $\phi_{1}$ and $\phi_{2}$ are thus distributed along the transverse axis of the burner, creating a linear variation in equivalence ratio. The flows then enter the first lateral mixing section (2a) consisting of a set of six, equally spaced, $10 \mathrm{~mm}$ wide, $152.4 \mathrm{~mm}$ long channels filled with $3 \mathrm{~mm}$ diameter glass beads. In this section of the burner, the two different streams mix laterally along the short axis (z-axis) of the burner. Each of the six channels thus has a different global mixture strength, which is linearly proportional to $\phi_{1}$ and $\phi_{2}$. To ensure a smooth gradient at the exit plane, the channels in the second lateral mixing section (2b) are offset by $5 \mathrm{~mm}$ (half their width) relative to the first mixing section. Finally, at the exit section of the burner (3), the flow is allowed to mix freely in the transverse and lateral directions to produce a smoothly varying gradient in equivalence ratio. 


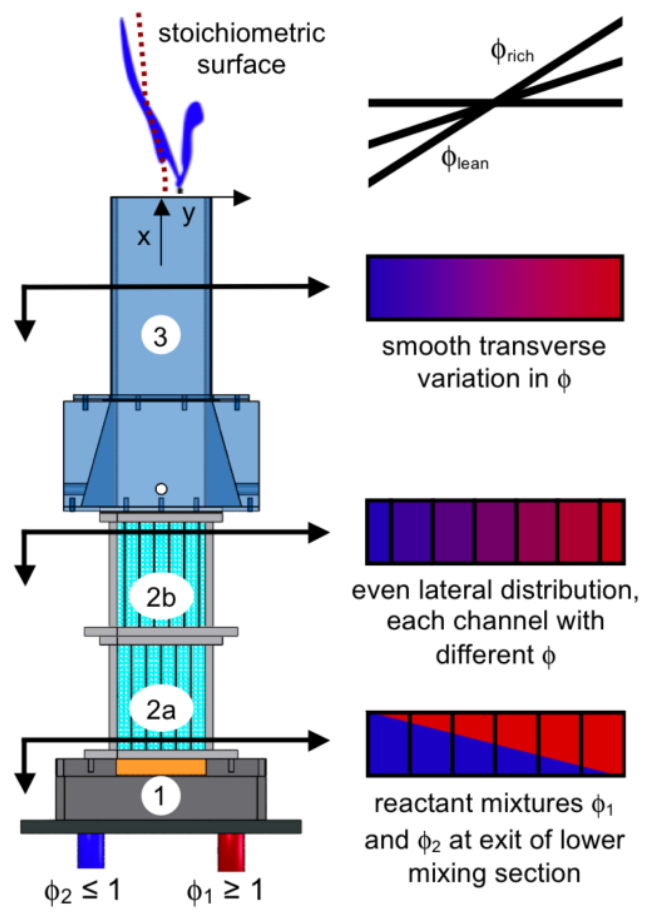

Figure 2.1 Reactant mixture progression through (1) lower, (2a,b) lateral, and (3) transverse mixing sections of the stratified burner.

The mixture strength and mean flow velocity are independently controlled using two pairs of digitally controlled Horiba LV-F liquid (iso-octane) and Brooks Smart-Series gas (air) mass-flow controllers. The mean flow velocity along $y= \pm 16 \mathrm{~mm}$ from the centerline of the burner was $4.6 \mathrm{~m} / \mathrm{s}$. Turbulence intensities were between 4.2-7.2\% within the same transverse range. Although integral length scales were not directly measured, they were projected to be in the range of 2 to $5 \mathrm{~mm}$ based on burners with a similar configuration and comparable flow settings $[39,45,50]$.

A $1.5 \mathrm{~mm}$ diameter rod was placed perpendicular to the flow direction $5 \mathrm{~mm}$ downstream of the exit plane at $y=16 \mathrm{~mm}$ from the center of the exit nozzle to stabilize the flame in a V-configuration. A continuous stratified flame front thus intersected the stoichiometric surface. Five flame conditions were considered by increasing the equivalence ratio in mixture $\phi_{1}$ from 1 to 1.8 on the rich end of the burner, while decreasing mixture $\phi_{2}$ from 1 to 0.2 on the lean end, in equivalence ratio steps of 0.2 .

Planar laser induced fluorescence (PLIF) was used for flow visualization of OH (hydroxyl) radicals and $\mathrm{CH}_{2} \mathrm{O}$ (formaldehyde) to obtain effectively instantaneous flame front topology measurements. Tracer PLIF of 3-pentanone $\left(\mathrm{C}_{2} \mathrm{H}_{5} \mathrm{COC}_{2} \mathrm{H}_{5}\right)$ was used for qualitative equivalence ratio measurements, and 
making laser profile corrections via imaging of a homogeneous reactant field. The laser diagnostic setup for simultaneous $\mathrm{OH}$ and $\mathrm{CH}_{2} \mathrm{O}$ PLIF shown in Figure 2.2 consists of an unseeded dual head Nd:YAG laser (Quanta Ray PIV-400) with independent crystals for doubling or tripling the fundamental frequency.

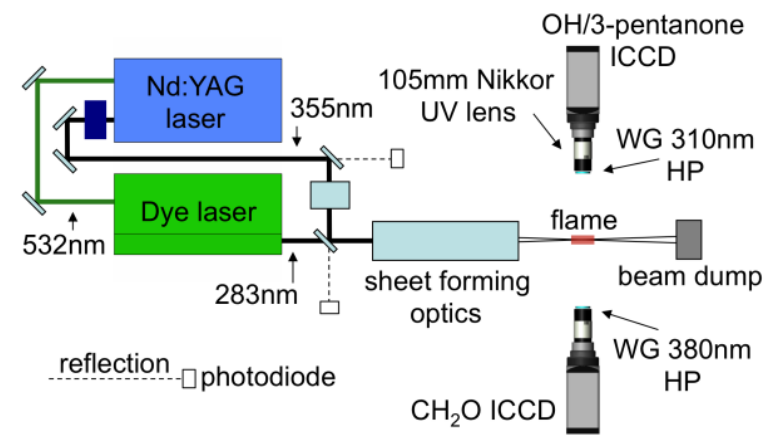

Figure 2.2 PLIF setup for simultaneous $\mathrm{OH}$ and $\mathrm{CH}_{2} \mathrm{O}$ acquisition.

The lower IR beam of the Nd:YAG laser, frequency tripled from its $1064 \mathrm{~nm}$ fundamental wavelength to the third harmonic at $355 \mathrm{~nm}\left(+/-3 \mathrm{~nm}, 300 \mathrm{~mJ}\right.$ per $7 \mathrm{nsec}$ pulse), was used for $\mathrm{CH}_{2} \mathrm{O}$. The upper IR beam was frequency doubled to $532 \mathrm{~nm}$ and subsequently used as the pump source for a Rhodamine-B dye-laser (Sirah Precision Scan). The tuneable output from the dye laser $(282.88 \mathrm{~nm}+/-$ $0.005 \mathrm{~nm}, 11 \mathrm{~mJ}$ per $6 \mathrm{~ns}$ pulse) was used to excite 3-pentanone as well as $\mathrm{OH}$ at the $\mathrm{Q}_{1}(6)$ line of the $(1,0)$ vibrational band of the $\mathrm{OH}$ A-X system. This produced $88.2 \mathrm{~mm}$ high x $500 \mu \mathrm{m}$ thick, $355 \mathrm{~nm}$ and $140.0 \mathrm{~mm}$ high $\mathrm{x} 500 \mu \mathrm{m}$ thick, $283 \mathrm{~nm}$ coincident laser sheets that intersected the central plane in the $\mathrm{z}-$ direction of the burner exit nozzle. A micro-lens array (beam homogenizer) was used for 3-pentanone images to ensure uniform laser intensity in the axial direction of the fluorescence images. The resulting laser sheet was $47.4 \mathrm{~mm}$ high $\mathrm{x} 500 \mu \mathrm{m}$ thick.

Fluorescence images were acquired using a pair of PIMAX 1340 x 1300 pixel intensified cameras positioned to provide a projected spatial resolution in the flame of $67 \mu \mathrm{m} / \mathrm{pixel}$. This equated to an effective field of view within the flame of $27.0 \mathrm{~mm}$ by $89.8 \mathrm{~mm}$ for 3-pentanone and $48.1 \mathrm{~mm}$ by $89.8 \mathrm{~mm}$ for $\mathrm{OH}$ and $\mathrm{CH}_{2} \mathrm{O}$. Precise timing of lasers and cameras was achieved using a DG-535 Stanford pulse generator. Flashlamp/Q-switch pulses for both lasers were phase shifted so that the $283 \mathrm{~nm}(\mathrm{OH} / 3-$ pentanone) and $355 \mathrm{~nm}\left(\mathrm{CH}_{2} \mathrm{O}\right)$ beams were delayed by $210 \mathrm{nsec}$. This phase delay was necessary to avoid 
overlap of the fluorescence signals induced by two laser sheets, and since $\mathrm{OH}$ has a shorter fluorescence lifetime than $\mathrm{CH}_{2} \mathrm{O}, \mathrm{OH}$ was excited first. Given the mean flow velocity of $4.6 \mathrm{~m} / \mathrm{s}$ and spatial resolution of $67 \mu \mathrm{m} / \mathrm{pixel}$, the reactants would propagate 0.01 pixels within this timeframe, and thus the $\mathrm{OH}$ and $\mathrm{CH}_{2} \mathrm{O}$ images can be treated as simultaneous realizations of the flow. Since flame topology is inherently three dimensional, two dimensional images may not fully capture the flow behaviour. However, planar measurements have been shown to provide an accurate depiction of curvature statistics [82]. Moreover, uncertainties are reduced when comparing among flame settings at a fixed region of interest in which the turbulent flow properties are held constant. Appendix B provides additional details and photographs of the experimental apparatus, dimensioned manufacturing drawings of the burner, and a full schematic of the isooctane/air delivery system feeding the burner.

\subsubsection{Reactant Characterization}

The equivalence ratio gradients were characterized for all flow settings under non-reacting conditions using tracer PLIF by seeding the iso-octane with 3 -pentanone at $7 \%$ by volume, often used for quantitative air/fuel ratio measurements [e.g. [83]] because its vapor properties match those of iso-octane and because its fluorescence signal is linearly dependant on concentration and laser intensity [84]. Calculations showed that the stoichiometric air to fuel ratios and lower heating values of the iso-octane/air reaction without and with added 3-pentanone at 7\% by volume were 59.5 (15.03 on a mass basis) vs. 57.7 (14.8 on a mass basis) and $44.7 \mathrm{MJ} / \mathrm{kg}$ vs. $43.3 \mathrm{MJ} / \mathrm{kg}$ respectively. The doping at $7 \%$ by volume ensured an adequate signal to noise ratio in the 3-pentanone PLIF images and limited the influence of the tracer on combustion. Ten images were acquired, each with 100 gates per exposure, equivalent to 1000 individual images per flow condition. The mean images were background corrected and subjected to a $3 \times 3$ median filter (equivalent to $0.20 \times 0.20 \mathrm{~mm}$ ), and the profiles in Figure 2.3 obtained by summing the rows of the corrected images corresponding to axial positions $\mathrm{x}=0.20-0.85 \mathrm{~mm}$ along the exit plane of the burner. The five different gradient profiles are all very smooth and intersect neatly at the stoichiometric surface where $\phi \approx 1$. Thus, by designing the experiments to track the location of this stoichiometric surface, it was possible to isolate and observe any effects on flame behaviour due to the presence of a mixture gradient about the stoichiometric contour. Since the mean exit velocity of the burner was fixed for all cases, the mean flow 
field and turbulence characteristics were also consistent within the region of interest (ROI) of the flame. The technique used to precisely define the region of interest is further explained below.

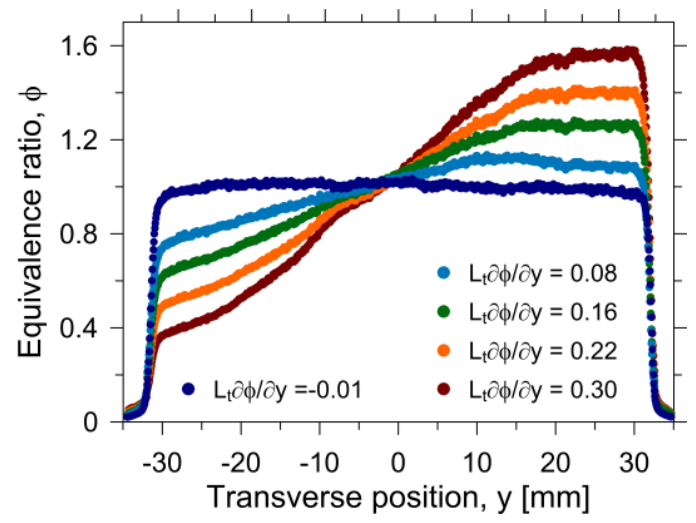

Figure 2.3 Transverse variation in equivalence ratio for five flame conditions with the gradient expressed in terms of turbulent flame brush thickness $L_{t}$.

Variations in the global equivalence ratio were within $\pm 5 \%$ of the mean for all flame settings and showed a maximum standard deviation of 0.07 between individual images. The mean profiles were in good agreement with expected gradient values with a standard deviation from 0.03 to 0.08 between individual profiles, measured transversely for $\mathrm{y}= \pm 15 \mathrm{~mm}$.

\subsubsection{Determination of the Location of the Stoichiometric Surface}

The position of the stoichiometric surface was identified under reacting conditions for each of the four stratified settings with single wavelength 3-pentanone tracer PLIF. One thousand individual images were acquired at each of four different axial positions $x=0.4-27.3 \mathrm{~mm}, \mathrm{x}=27.3-54.2 \mathrm{~mm}, \mathrm{x}=54.2-81.1 \mathrm{~mm}$, and $\mathrm{x}=81.1-108.1 \mathrm{~mm}$. Laser energy was adjusted to ensure fluorescence linearity throughout the entire laser sheet while simultaneously accounting for pulse-to-pulse variations in laser intensity, permitting semiquantitative measurements of average mixture strength in the unburnt gases. Individual 3-pentanone PLIF images were corrected for background intensity and laser profile variations before ensemble averaging. A $3 \times 3$ pixel median filter was applied to the ensemble average image to eliminate any residual shot noise. Configuration and calibration procedures, as well as a flow chart outlining each step of the 3-pentanone PLIF image processing routine (including sample images) are presented in Appendix C. The resultant image was then conditioned on reactants using a map of the reactant progress variable derived from an 
ensemble average of a binarized version of the same 3-pentanone images. Image sets from the four different heights were combined to generate continuous maps of reactant 3-pentanone concentrations that were used to evaluate the local mixture strength within the reactants up to approximately $11 \mathrm{~cm}$ above the burner exit nozzle. Figure 2.4 shows a sample final reactant concentration image for $\mathrm{L}_{t} \delta \phi / \delta y=0.22$.

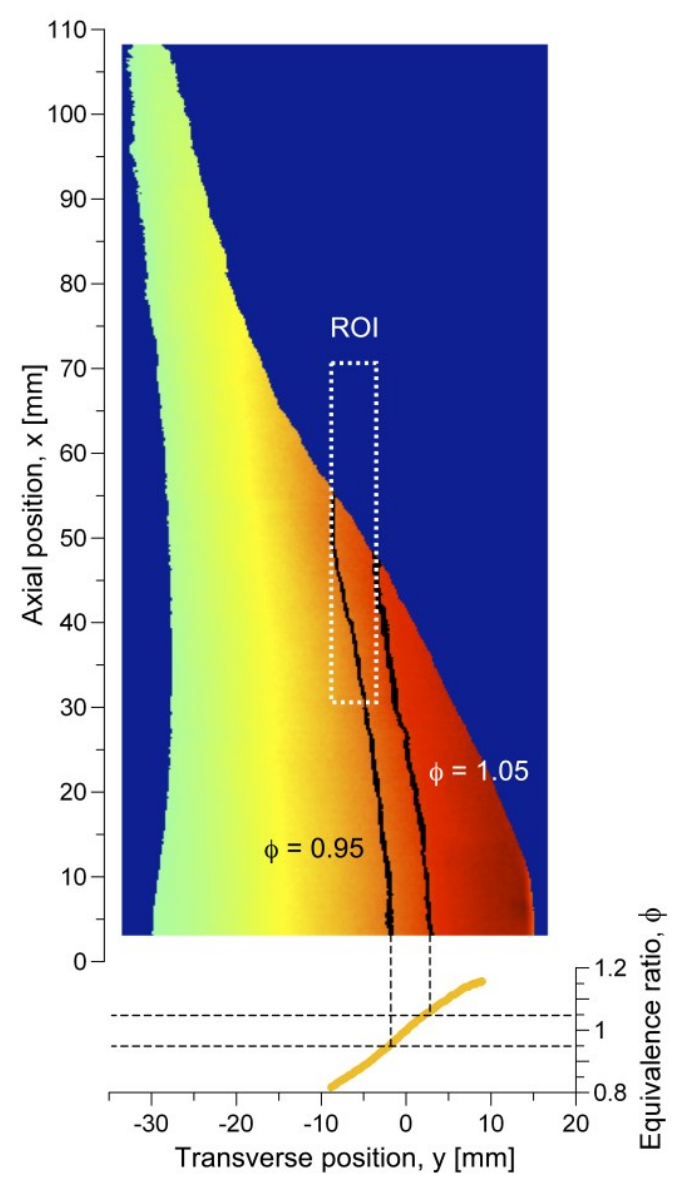

Figure 2.4 Mixture strength distribution and corresponding interrogation window for stratified flame $L_{t} \delta \phi / \delta y=0.22$.

Similar concentration images were derived for each stratified flame condition and were used to identify the location of the stoichiometric surface as well as the average mixture strength at the mean position of the flame front, $\langle\mathrm{c}\rangle=0.5$, within the turbulent flame brush. To permit fair comparison among different conditions and specifically to isolate the effects of equivalence ratio gradient from effects of local mixture strength, it was necessary to maintain a constant range of equivalence ratios within the analysis region of the flame. This was accomplished by varying the width of the region of flame being analyzed, 
indicated by the superimposed dashed white box in Figure 2.4. The width of this box was precisely adjusted for different flame conditions to ensure that the range of equivalence ratios it contained was held constant between $0.95 \leq \phi \leq 1.05$ for all flames. Figure 2.4 also shows the corresponding $\phi=0.95$ and $\phi=1.05$ surfaces used to define the interrogation region for the $L_{t} \delta \phi / \delta y=0.22$ flame condition. By carefully specifying the widths of these analysis regions, it was possible to assume the flames within were propagating under essentially identical mean flow and equivalence ratio conditions, while being subjected to widely differing gradients. The length scales of the large-scale gradients (defined as the width of the analysis region, $0.95 \leq \phi \leq 1.05$ ) varied from 3.5 to $12.5 \mathrm{~mm}$.

\subsection{Results and Discussion}

One thousand individual $\mathrm{OH}$ and $\mathrm{CH}_{2} \mathrm{O}$ PLIF images were acquired for each of the five flame configurations. The $\mathrm{CH}_{2} \mathrm{O}$ images were registered to the $\mathrm{OH}$ images using a 90 point target to spatially match the corresponding pixels of each camera to ensure they imaged identical positions within the flame. As was the case for 3-pentanone, images were corrected for background as well as laser profile, and subjected to a $3 \times 3$ median filter to reduce shot noise. Configuration and calibration procedures, as well as flow charts showing sample instantaneous $\mathrm{OH}$ and $\mathrm{CH}_{2} \mathrm{O}$ PLIF images corresponding to each image processing operation are provided in Appendix C. Images were evaluated to quantify the effect of gradient on turbulent brush thickness and orientation, flame surface density, and curvature.

Through analysis of the instantaneous 3-pentanone PLIF data, variation in scale in intensity of the local fluctuations in equivalence ratio could be quantified as the large-scale gradient was varied. The local fluctuations occurred on length scales associated with those expected for turbulent eddies (i.e. $0.5-3 \mathrm{~mm}$ ), and did not vary as the global gradient was changed.

\subsubsection{Progress Variable}

Figure 2.5 shows a flood plot of the ensemble averaged reaction progress variable $\langle\mathrm{c}\rangle$, obtained from binarized $\mathrm{OH}$ images, in the vicinity of the stoichiometric contour for gradient setting $\mathrm{L}_{t} \delta \phi / \delta y=0.16$. The

image represents a $48.1 \mathrm{~mm} \times 39.6 \mathrm{~mm}$ area within the flame, where dark blue corresponds to fresh reactants $(\langle c\rangle=0)$ and dark red to pure products $(\langle c\rangle=1)$. As the mean flame front in Figure 2.5 crosses the 
stoichiometric surface, it recedes downstream, an effect also observable in Figure 2.4. Because the mixture strength diminishes away from stoichiometric, the local flame speed decreases and causes iso-contours of $\langle c\rangle$ to deviate from their initial incident angle in the vicinity of the stabilizing bar. This behaviour was not observed for the homogeneous case, in which the orientation of the flame brush did not change relative to the mean flow direction. These observations further validate the numerical predictions of Robin et al. [29] for methane/air V-flames.

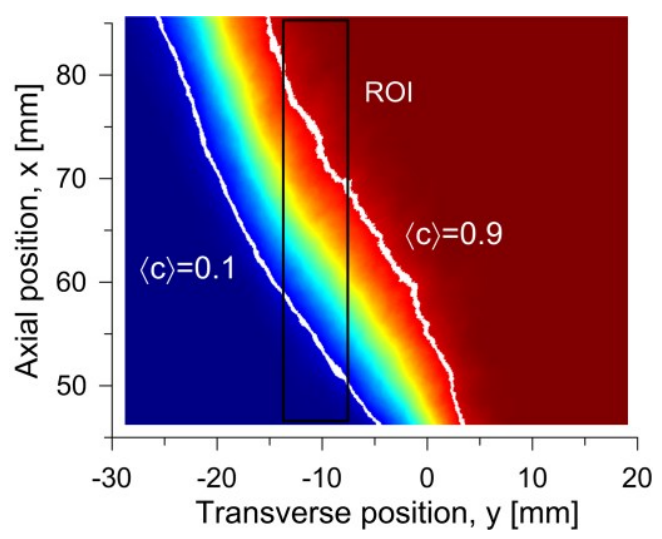

Figure 2.5 Progress variable $\langle c\rangle$ map and corresponding interrogation window for stratified flame $\mathbf{L}_{\mathrm{t}} \delta \phi / \delta \mathbf{y}=0.16$.

\subsubsection{Flame Surface Density}

The two dimensional flame surface density (FSD) data were calculated with the approach outlined in Deschamps et al. [85] where $\Sigma$ is derived from the definition of Pope [86], $\Sigma=\left\langle\Sigma^{\prime}\right\rangle=\left\langle|\nabla c| \cdot \delta\left(c-c_{\mathrm{f}}\right)\right\rangle$, for which \langle\rangle denotes the ensemble average of the enclosed quantity, $|\nabla c|$ is the absolute value of the spatial flame front gradient, $\delta\left(\mathrm{c}-\mathrm{c}_{\mathrm{f}}\right)$ the instantaneous flame position, and $\delta$ the Kronecker delta. The instantaneous FSD, $\Sigma^{\prime}$, was obtained from flame contours derived from individual OH PLIF images where the position of the flame front was determined by thresholding. The instantaneous $\Sigma^{\prime}$ images were then masked by multiplying with the matching binary $\mathrm{CH}_{2} \mathrm{O}$ PLIF image. This approach was particularly useful in processing severely stratified cases, in which regions of weaker $\mathrm{OH}$ signal behind the flame front were filtered out, creating non-physical edges once thresholding was applied. This approach was derived from the method of Paul and Najm [55] for heat release rate calculations, who determined that the product of $\mathrm{OH}$ and $\mathrm{CH}_{2} \mathrm{O}$ was a better approximation of the position of the flame front than $\mathrm{OH}$. Although the $\mathrm{CH}_{2} \mathrm{O}$ 
images, and resulting heat release rate contours, were not as clearly defined as those observed by Balachandran et al. [58] and Pfadler et al. [87], and therefore too noisy to accurately define the position of the flame front, they were effective in masking the $\Sigma^{\prime}$ images.

The instantaneous, masked $\Sigma^{\prime}$ images were then ensemble averaged and plotted in terms of $\langle\mathrm{c}\rangle$. The FSD profiles shown in Figure 2.6 are slightly skewed with maximum values falling consistently toward the burnt gases, in the vicinity of $\langle c\rangle=0.6$. The asymmetric profiles were consistent with those of turbulent V-flames observed by Shepherd [88] for premixed methane/air and ethylene/air, and AnselmoFilho et al. [45] for partially-premixed methane/air mixtures, such that the $\mathrm{k}\langle\mathrm{c}\rangle(1-\langle c\rangle)$ expression, used for chemical closure models for fractal flamelets [89], does not provided a good estimate of $\Sigma(\langle c\rangle)$. Shepherd [88] proposed that the ordered, cusped structure of the V-flame towards the burnt gases contributes to the asymmetry in FSD, as confirmed by their observation of bimodal PDFs of flame orientation.

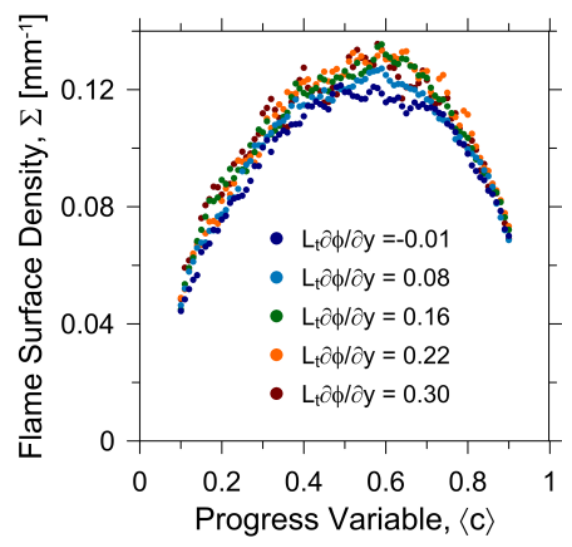

Figure 2.6 Flame surface density for premixed and stratified flames.

Although the bias of FSD profiles did not vary among conditions, comparing the homogeneous and stratified profiles reveals a non-linear increase in FSD with increasing gradient. The effect is strongest for the weaker gradients but levels off for the severely stratified cases. This can be expected from a qualitative visual inspection of individual fluorescence images, in which stronger mixture gradients were associated with significant increases in flame front wrinkling and corrugation, ultimately leading to a sharp reduction in the instantaneous $\mathrm{OH}$ fluorescence signal. Although the measured flame brush thickness 
(defined as the perpendicular distance between $\langle\mathrm{c}\rangle=0.1$ and $\langle\mathrm{c}\rangle=0.9$ contours) was consistently greater with stratification ( 10.5 mm compared to $9.5 \mathrm{~mm}$ for the homogeneous flame at the same axial position $\mathrm{x}=45 \mathrm{~mm}$ above the exit of the burner), it did not change significantly as the gradient was increased. This implies that the dominant effect of large-scale mixture gradients may be to enhance the number and intensity of wrinkles, similar to the reported effects of small-scale inhomogeneities in mixture fraction $[16,17]$.

\subsubsection{Curvature}

Curvature measurements were obtained by fitting a cubic spline to the leading edge of the instantaneous binary flame images. The local curvature $\kappa$ was calculated from $\kappa=(\dot{x} \ddot{y}-\dot{y} \ddot{x}) /\left(\dot{x}^{2}+\dot{x}^{2}\right)^{3 / 2}$, and by convention, positive curvatures are defined as convex towards the reactants and negative curvatures are defined as concave towards the reactants. The length-weighted PDFs of curvature for each flame setting were calculated by normalizing the number of observations in each bin by the total number of local curvature measurements and plotted in Figure 2.7. The curvature PDFs were nearly symmetrical, with a slight bias toward the reactants. This is consistent with the FSD results of Figure 2.6, and the generally accepted trend for cusped flame fronts, where the larger area of positive fronts results in the biased curvature plots. The shift is further emphasized by the negligible flame area of infrequent cusps, which manifest themselves as large negative curvatures [90].

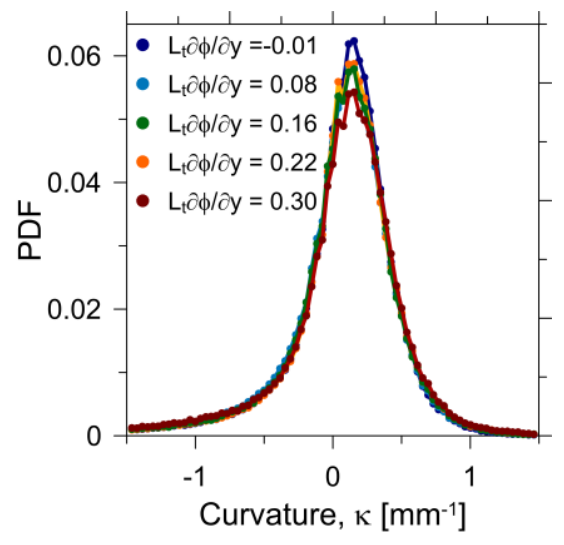

Figure 2.7 Comparison of curvature PDFs for globally stoichiometric premixed and partiallypremixed iso-octane/air V-flames. 
The most probable curvature was consistently around $\kappa \approx 0.2 \mathrm{~mm}^{-1}$, and the probability of encountering this level of curvature decreased only slightly for stronger gradients, contrary to observations of Anselmo-Filho et al. [45] for whom the bias of the profiles changed with apparent gradient. Furthermore, no significant increase in variance with stratification was observed in the present results.

The apparent discrepancy between the present topology results and those of Anselmo-Filho et al. [45] using methane under globally lean conditions can arguably be attributed to differences in mixture strength, methodology, and fuel type. Firstly, as suggested by Zhou et al. [17], stratification has a greater effect on flame propagation for off-stoichiometric mixtures, since the thermo-diffusive stability of stoichiometric flames limits the effects of stratification. Secondly, the variable interrogation windowing technique used in the present analysis to freeze the range of equivalence ratios (and hence Lewis numbers which would affect the wrinkling scales of the flame front) may at least partially affect the interpretation of flame topology. Finally, because of the significant difference in the Markstein numbers for iso-octane/air versus methane/air flames [91], it is reasonable to expect that response to perturbations from the stabilizing rod could be different in the two cases, again affecting the significance or magnitude of observed differences in flame topology with increased mixture gradient.

\subsection{Conclusion}

The effects of large-scale gradients in equivalence ratio on locally stoichiometric turbulent iso-octane/air V-flames were studied using a novel stratified burner capable of producing transverse variations in mixture strength. PLIF images of $\mathrm{OH}, \mathrm{CH}_{2} \mathrm{O}$, and 3-pentanone were acquired for five flame conditions to evaluate variations in flame front topology and to quantify these differences through flame surface density and curvature measurements. Critical to interpreting the data was the technique of using variable width interrogation windows to fix the range of equivalence ratios being considered when comparing flames subjected to different mixture gradients.

Gradients in equivalence ratio had a dramatic effect on flame wrinkling, leading to enhanced corrugation of the flame front for the strongest gradients. However, the effect of increased flame surface density was more modest, balanced in part by an increase in flame brush thickness, and ultimately by a decrease in flame length. Perhaps surprisingly, variations in curvature distribution were nearly negligible 
and not nearly as prominent as those reported in the literature for lean methane/air V-flames. This suggests that although gradients in mixture strength may alter the overall structure and instantaneous behaviour of globally stoichiometric combustion systems, their effect on the topology of locally stoichiometric flames may be limited, suggesting that premixed models for turbulent combustion are adequate for the modeling of near stoichiometric, stratified flames. 


\section{Chapter 3}

\section{Heat Release Rate Variations in a Globally Stoichiometric, Stratified Iso-Octane/Air Turbulent V-Flame}

This chapter is currently under peer-review for possible publication in Combustion and Flame. It should be cited as:

Vena, P.C., Deschamps B., Guo, H., Smallwood, G.J., Johnson, M.R. (2014) "Heat release rate variations in a globally stoichiometric, stratified iso-octane/air turbulent V-flame", submitted to Combustion and Flame and under consideration for publication (submitted December 13, 2013; manuscript id: CNF-D-13-00630).

This paper was co-authored by the thesis author, Patrizio Vena, Dr. Béatrice Deschamps, Dr. Hongsheng Guo, Dr. Greg Smallwood, and Prof. Matthew Johnson. Mr. Vena set up, tested, and characterized the measurement equipment; performed the measurements, uncertainty analysis, and initial data analysis; and wrote a first draft of the manuscript. Dr. Deschamps helped in the design of the experiments. Dr. Guo conducted the numerical simulations. Dr. Smallwood participated in the initial design of the burner, provided access to laboratory facilities, and shared in funding the project. The theory development, final data analysis, and editing and revision of the manuscript were conducted jointly by Mr. Vena and Prof. Johnson.

\subsection{Abstract}

Combustion intensity variations along a globally stoichiometric, stratified iso-octane/air turbulent V-flame were measured in the presence of four different equivalence ratio gradients and compared to a reference fully-premixed case. Instantaneous heat release rate (HRR) images were obtained from the simultaneous acquisition of $\mathrm{OH}$ and $\mathrm{CH}_{2} \mathrm{O}$ Planar Laser Induced Fluorescence (PLIF) images, providing the first direct estimates of local heat release rate in a stratified, iso-octane/air turbulent flame. Detailed numerical simulations were conducted to validate the use of $(\mathrm{OH} \mathrm{LIF})\left(\mathrm{CH}_{2} \mathrm{O}\right.$ LIF) for measurements of HRR in isooctane/air flames over a broad range of equivalence ratios. Flame images were analyzed for specific mean equivalence ratio gradient effects for near stoichiometric flame regions within a fixed mean equivalence ratio range of $0.95 \leq \phi \leq 1.05$. The location of the interrogation window, or region of interest (ROI), was 
obtained through 3-pentanone tracer PLIF of the reactants and ensured that any observed variation could be attributed specifically to mean equivalence ratio gradient effects, rather than simple mixture strength effects. Results showed small but measureable decreases in the mean HRR for near stoichiometric flame regions within the ROI as the gradient was varied, and differences of up to $4.4 \%$ relative to the reference premixed case were directly attributable to the imposed mean $\phi$ gradient, separate from potential effects of local mixture strength. By contrast, general stratification effects were also considered for flames of comparable global equivalence ratios but with much broader ranges of mixture strength, and results showed offsetting effects of increased 2D flame length (up to $17 \%$ ) and decreased HRR per flame length (up to $\sim 22 \%$ ), resulting in small variations in the overall HRR of $\sim 9 \%$. The present analysis suggests that gradient effects on local flame properties may differ significantly from general stratification effects in which different ranges of mixture strengths are present, and separating these aspects of partially premixed combustion is critical to the proper interpretation of data, where both may be relevant in different applications.

Keywords: Stratified combustion, Turbulent combustion, V-flame, Equivalence ratio gradient, Planar Laser Induced Fluorescence, Heat release rate 


\subsection{Introduction}

As stratified charge engines and partially premixed combustors emerge as viable approaches for reducing fuel consumption $[8,11,12,92]$ and pollutant emissions $[8,11]$, better data and understanding of stratified flames are required to further technology development. Controlled experimental investigation of the effects of partial premixing on flame behaviour is essential to improving theoretical understanding and relating stratified flame dynamics to classical models for premixed and diffusion flames.

Stratified combustion has received considerable attention in recent years, and although broader theoretical understanding of the effects of stratification on flame propagation is still emerging, there are at least three generally accepted observations [29]. First, stratified flames may be "back supported" by heat and radicals as they propagate in the direction of the gradient vector through large-scale variations in equivalence ratio, resulting in a greater flame propagation velocity as well as an extension of flammability limits $[21-26,70,72,80]$. Secondly, local variations in flame speed, $S_{L}$, caused by heterogeneities in equivalence ratio act as an additional mechanism for turbulent wrinkling of the flame front in cases where $\mathrm{u}^{\prime} / \mathrm{S}_{\mathrm{L}}^{\mathrm{o}}<1[39,40,66]$ with the effect less significant as $\mathrm{u}^{\prime} / \mathrm{S}_{\mathrm{L}}^{\mathrm{o}}$ increases $[39,66-69]$. This alters the flame's structure and topology, including flame thickness and rate of strain [29-38], flame length and wrinkling scales $[4,16,17,29,30,39-46]$, flame surface density [31,34,45,47,48], and curvature [29-35,41,45-47]. Thirdly, and the focus of this paper, stratification leads to variations in the local combustion intensity along a reacting front $[39,40,66-70,72,80]$, which may in turn lead to differences in global reaction rate of the flame $[39,40,66-69]$.

Several computational studies have evaluated the effects of partial premixing on combustion intensity. Numerical works by Marzouk et al. [70], Richardson et al. [72], and Zhou and Hochgreb [80] studied the effects of equivalence ratio gradients in counterflow flames. Enhanced burning rates [70] and heat release rates $[72,80]$ have been observed in lean back supported flames, while a decrease in heat release rate has been observed for front supported flames [72,80]. Direct numerical simulations (DNS) of 1D [66,67], 2D [39,66-68] and 3D [66,69] flames in which an unstrained premixed flame is propagated into a stratified mixture have evaluated the effects of the length scale [68,69], spatial distribution [68], and scalar distribution [39,66-69] of the fluctuations in mixture strength at different levels of turbulence and 
mean equivalence ratios. While predictions consistently showed local variations in combustion intensity along the flame front, the net effects of stratification on the overall combustion intensity, defined by flame surface generation and the reaction rate per unit flame length, have revealed differing trends among conditions. Authors have reported increases $[39,68]$, decreases $[39,68,69]$, or negligible changes $[66,67]$ in total combustion intensity, with occasionally contradictory results for similar conditions [16,20 vs 18$]$.

The experimental works of Zhou et al [17] and Cho and Santavicca [16] have implied variations in global reaction rate with stratification, reporting on differences in flame surface wrinkling and mean propagation velocity. Although their results proved insightful, they did not specifically evaluate the effect of stratification on the combustion intensity along the flame front.

Building on previous work that investigated variations in flame surface density and curvature with partial premixing [47], the key objective of the present effort is to quantify the local variations in heat release rate along a turbulent stratified flame front in the presence of varied gradients in equivalence ratio. While heat release rate measurements have been successfully performed on a variety of premixed flames (e.g. laminar counterflow flames [56], turbulent counterflow flames [57], turbulent bluff body stabilized flames [57,58], turbulent swirl flames [59-61], cone-shaped flames [62], laminar V-flames [63]) and lifted jet flames $[64,65]$, to the authors' knowledge, the data presented here are the first direct measurements of local combustion intensity along a stratified flame front in which the $\phi$ gradient is explicitly controlled, providing insight into the underlying mechanisms that govern stratified flame behaviour.

A globally stoichiometric iso-octane/air V-flame was stabilized under fully- and partiallypremixed conditions, with baseline measurements for the zero stratification case compared to results at four different mean equivalence ratio gradients along the flame. A novel approach to defining the location of the flame front was developed that accounted for the presence of local discontinuities when flame extinctions were evident. Local combustion intensities were measured using the approach proposed by Paul and Najm [55], in which the product of $\mathrm{OH}$ and $\mathrm{CH}_{2} \mathrm{O}$ fluorescence is correlated to the local heat release rate along a flame front, marking the first implementation of this diagnostic to stratified, turbulent iso-octane/air flames. Results of these measurements were first used to examine stratification effects on $\mathrm{OH}$ and $\mathrm{CH}_{2} \mathrm{O}$ fluorescence intensities within instantaneous flame images and corresponding local 
$(\mathrm{OH} \mathrm{LIF})\left(\mathrm{CH}_{2} \mathrm{O}\right.$ LIF) heat release rates. Large-scale gradient effects on mean flame heat release rates were then evaluated using a windowing approach introduced in [47]. This ensured fair comparison between premixed and stratified gradient cases by maintaining a constant mean range of equivalence ratios within the analysis region, such that the effects of equivalence ratio gradients on locally stoichiometric flame regions could be directly compared and quantified. This approach allowed distinction between the specific effects of mean gradients in equivalence ratio from those of varying mixture strength. Global stratification effects were separately considered by comparing the total heat release rates of stoichiometric premixed flames and globally stoichiometric stratified flames, in which the range of equivalence ratios varied between gradient settings.

\subsection{Experimental Setup and Methodology}

Stratified iso-octane/air V-flames were stabilized above a unique $63 \mathrm{~mm}$ x $15 \mathrm{~mm}$ rectangular exit burner, shown schematically in Figure 3.1. The burner permits transverse variation of equivalence ratio at its exit plane. Two premixed reactant streams of different equivalence ratios $\phi_{1}$ and $\phi_{2}$ enter separate compartments of the $32 \mathrm{~mm}$ high base section of the burner (section 1 in Figure 3.1). The reactant streams then flow through separate, triangular expanded metal foam inserts $(15 \mathrm{~mm} \times 65 \mathrm{~mm} \times 12.7 \mathrm{~mm})$ that are sealed from one another to prevent mixing (seen in cut 1 in Figure 3.1). The two streams then enter the mixing section 2a, which is $88.9 \mathrm{~mm}$ high and subdivided into six $10 \mathrm{~mm}$ wide channels, each filled with $3 \mathrm{~mm}$ glass beads. The triangularly shaped inlet sections result in different proportions of the reactant streams entering each of the six channels in the mixing section. Within each channel, the reactant streams $\phi_{1}$ and $\phi_{2}$ are allowed to fully mix in the lateral z direction (shown as cut 2), such that the flows exiting these channels create a step-wise equivalence ratio gradient along the y-axis of the burner. The reactant flows then enter mixing section $2 \mathrm{~b}$, also $88.9 \mathrm{~mm}$ in height, in which the channels are offset by half the channel width $(5 \mathrm{~mm})$ relative to those of section 2a. This acts to smooth the steps in equivalence ratio by facilitating transverse mixing in the y direction. Finally, the flow is then allowed to mix freely in the $225 \mathrm{~mm}$ high exit section (section 3), generating a smooth gradient in equivalence ratio at the exit plane of the burner. A $4 \mathrm{~mm}$ air co-flow surrounds the exit nozzle. 


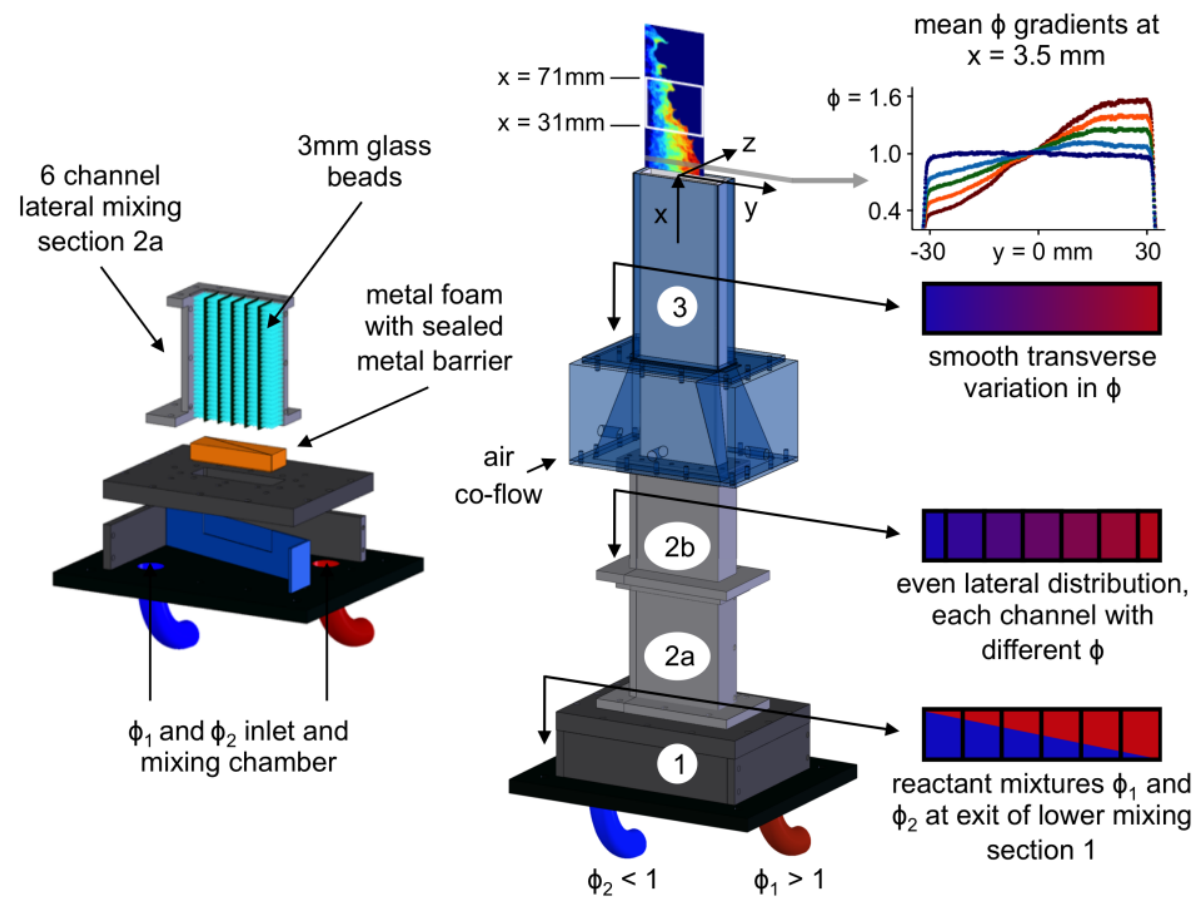

Figure 3.1 Reactant mixture progression through (1) lower, (2a, 2b) lateral, and (3) transverse mixing sections of the stratified burner. A sample instantaneous equivalence ratio image is shown between axial positions $x=0-120 \mathrm{~mm}$ above the exit plane of the burner for stratified flame condition $\delta \phi / \delta y=0.021 \mathrm{~mm}^{-1}$, where the white rectangle identifies the region between $\mathrm{x}=31-71 \mathrm{~mm}$ where $\mathrm{OH}$ and $\mathrm{CH}_{2} \mathrm{O}$ PLIF images were acquired.

Reactant streams $\phi_{1}$ and $\phi_{2}$ were independently regulated with two pairs of digitally controlled Brooks Smart-Series air (5853S/BE 200 SLPM full scale) and Horiba LV-F liquid (rich side: LF-F60MO $40 \mathrm{ccm}$ iso-octane full scale, lean side: LF-F50MO-094 $25 \mathrm{ccm}$ iso-octane full scale) mass flow controllers. The reactant air was preheated with Omegalux AHP-7561 in-line air heaters to $80^{\circ} \mathrm{C}$, resulting in a reactant temperature of $55^{\circ} \mathrm{C}$ at the exit of the burner (measured along the exit plane of the burner using a type- $\mathrm{K}$ thermocouple). Modifications to the fuel injection system were made relative to that initially described in [47] to ensure proper mixing of the liquid fuel with air. Iso-octane was introduced in the air lines 3 meters upstream of lower mixing section 1, rather than directly at the burner inlet, to enhance premixing of reactant streams $\phi_{1}$ and $\phi_{2}$.

The Planar Laser Induced Fluorescence (PLIF) experimental setup shown in Figure 3.2 consists of a dual head Quanta Ray PIV400 Nd:YAG coupled with a Sirah Precision Scan Rhodamine B dye laser. The first IR beam of the Nd:YAG was frequency doubled to $532 \mathrm{~nm}$ and used to pump the dye laser, which 
outputs a $283 \mathrm{~nm}$ narrowband tunable beam $( \pm 0.005 \mathrm{~nm}, 11 \mathrm{~mJ}$ per $6 \mathrm{~ns}$ pulse $)$. The $283 \mathrm{~nm}$ beam was then expanded vertically though a $-100 \mathrm{~mm}$ focal length cylindrical lens and directed through a micro-lens array (beam homogenizer), through the back surface of a $355 \mathrm{~nm}$ mirror (CVI coating 43-1037-45), and finally through a $1000 \mathrm{~mm}$ spherical converging lens to produce a $47.4 \mathrm{~mm}$ high $\mathrm{x} 250 \mu \mathrm{m}$ thick laser sheet. The second IR beam of the Nd:YAG was frequency tripled to $355 \mathrm{~nm}$ broadband $( \pm 3 \mathrm{~nm}, 300 \mathrm{~mJ}$ per $7 \mathrm{~ns}$ pulse) and expanded vertically through a $-100 \mathrm{~mm}$ focal length cylindrical lens, reflected off the front surface of the same $355 \mathrm{~nm}$ mirror, and directed through the same $1000 \mathrm{~mm}$ spherical lens to form a $88.2 \mathrm{~mm} \times 250 \mu \mathrm{m}$ sheet that was coincident with the $283 \mathrm{~nm}$ sheet.

The beam homogenizer generated a uniform laser intensity profile in the axial x-direction for the $283 \mathrm{~nm}$ beam. See [87] for a detailed description of the underlying principles of the micro-lens array. However, the low damage threshold of the homogenizer meant it could not be used for the higher energy $355 \mathrm{~nm}$ beam. The pulse to pulse variations in laser energy were measured with a pair of Thorlabs DET10A Si-based high speed photodiodes. The analogue photodiode signal was hardware integrated using a Stanford Research Systems SR280 Boxcar Averager System outfitted with two SR250 Boxcar Gated Integrator Modules. Care was taken to minimize saturation of the photodiodes by selecting a reflection of limited energy density to ensure the integrated signal resembled the expected Gaussian temporal profile of the laser output.

The $283 \mathrm{~nm}$ sheet was used to excite $\mathrm{OH}$ at $282.94 \mathrm{~nm}\left(\mathrm{Q}_{1}(6)\right.$ line of the $(1,0)$ vibrational band of the $\mathrm{OH}$ A-X system). During local equivalence ratio measurements, the $283 \mathrm{~nm}$ sheet was also used to excite 3-pentanone $\left(\mathrm{C}_{2} \mathrm{H}_{5} \mathrm{COC}_{2} \mathrm{H}_{5}\right)$ at $282.75 \mathrm{~nm}$. The $355 \mathrm{~nm}$ sheet was used to excite formaldehyde $\left(\mathrm{CH}_{2} \mathrm{O}\right)\left(\widetilde{\mathrm{A}}^{1} \mathrm{~A}_{2}-\widetilde{\mathrm{X}}^{1} \mathrm{~A}_{1} 4_{0}^{1}\right.$ vibronic manifold $\left.[93,94]\right)$ during flame experiments, and separately used to excite Biacetyl $\left(\mathrm{CH}_{3} \mathrm{COCOCH}_{3}\right)$ during configuration experiments to establish laser profile corrections. Fluorescence data were acquired using two Princeton Instrument PIMAX 1340 x 1300 pixel Intensified CCDs and ST-133 controllers. OH/3-pentanone PLIF images were captured using an ICCD equipped with a Nikkor $105 \mathrm{~mm}$ UV lens and WG $310 \mathrm{~nm}$ high-pass (HP) filter to reject Rayleigh scattering from the $283 \mathrm{~nm}$ laser sheet. $\mathrm{CH}_{2} \mathrm{O} /$ Biacetyl PLIF images were recorded on a second ICCD equipped with an identical Nikkor lens and a WG $380 \mathrm{~nm}$ HP filter, to reject Rayleigh scattering from the $355 \mathrm{~nm}$ laser sheet. 
The cameras and associated lenses were positioned to image a $40.0 \mathrm{~mm}$ by $89.9 \mathrm{~mm}$ field of view within the flame at a projected spatial resolution of $67 \mu \mathrm{m} /$ pixel.

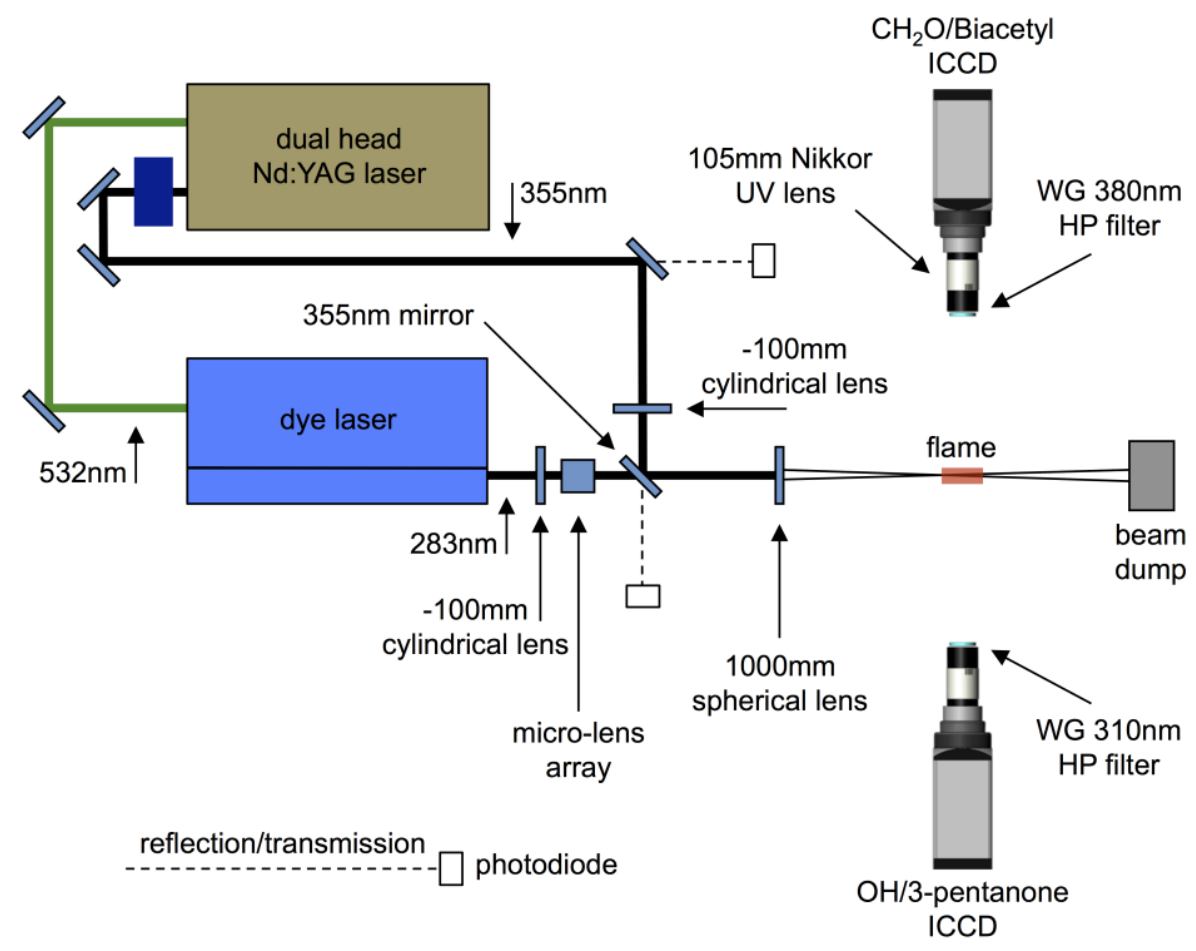

Figure 3.2 Planar laser induced fluorescence setup for simultaneous $\mathrm{OH}$ and $\mathrm{CH}_{2} \mathrm{O}$ acquisition.

Near-simultaneous acquisition of $\mathrm{OH}$ and $\mathrm{CH}_{2} \mathrm{O}$ was achieved by delaying the $\mathrm{CH}_{2} \mathrm{O}$ laser pulse by $400 \mathrm{nsec}$ relative to the $\mathrm{OH}$ laser pulse using a Stanford DG-535 Stanford pulse generator to avoid overlap of the $\mathrm{OH}$ and $\mathrm{CH}_{2} \mathrm{O}$ fluorescence signals. $\mathrm{OH}$ was excited first because of its relatively short fluorescence life time. At the maximum expected mean velocity of $5.5 \mathrm{~m} / \mathrm{sec}$, the flow would be expected to move $2.2 \mu \mathrm{m}$ (equivalent to approximately 0.03 pixels) between the $\mathrm{OH}$ and $\mathrm{CH}_{2} \mathrm{O}$ excitations, resulting in effectively simultaneous imaging of both species in the flow field.

The turbulent V-flame was stabilized with a $1.5 \mathrm{~mm}$ diameter rod aligned with the $\mathrm{z}$-axis of the burner and positioned at $\mathrm{x}=5 \mathrm{~mm}$ and $\mathrm{y}=16 \mathrm{~mm}$ relative to an origin centered in the burner exit plane for all flame conditions. Five flame conditions were studied by varying the equivalence ratio gradient at the exit of the burner from $\partial \phi / \partial y=-0.001 \mathrm{~mm}^{-1}$ to $\partial \phi / \partial y=-0.029 \mathrm{~mm}^{-1}$. The exit plane velocity and turbulence intensity profiles were characterized using a Dantec Dynamics Laser Doppler Velocimetry 
(LDV) system under non-reacting conditions with olive oil seeding. Data were acquired in the $\mathrm{x}, \mathrm{y}$, and $\mathrm{z}$ directions and showed negligible velocities in the transverse $\mathrm{y}$ and lateral $\mathrm{z}$ directions. In addition, potential entrainment of the surrounding air was investigated and verified to be negligible following an analysis of the instantaneous and ensemble-averaged 3-pentanone fluorescence images, for the non-reacting homogeneous reference case. Results showed that the pixel intensities remained constant from the exit plane of the burner up to an elevation of roughly $80 \mathrm{~mm}$, and thus were unaffected by ambient air entrainment.

Figure 3.3 shows mean velocity and turbulence profiles along the centre plane of the burner $(\mathrm{z}=0)$, at an axial position $\mathrm{x}=5 \mathrm{~mm}$ above the burner exit plane. The mean flow velocity, $\overline{\mathrm{U}}$, was $5.2 \mathrm{~m} / \mathrm{s}$ and varied by less than $8 \%$ within $40 \mathrm{~mm}$ along the burner $(\mathrm{y}= \pm 20 \mathrm{~mm})$. The turbulence intensity ranged between $3.7 \%$ and $7.7 \%$ within the same transverse positions. For a reference unstrained laminar flame speed of $\mathrm{S}_{\mathrm{L}}^{\mathrm{o}}=37.8 \mathrm{~cm} / \mathrm{sec}$ estimated from [95-99] at a reactant temperature of $55^{\circ} \mathrm{C}$ at the exit of the burner, this equates to $0.51 \leq \mathrm{u}^{\prime} / \mathrm{S}_{\mathrm{L}}^{\mathrm{o}} \leq 1.06$.

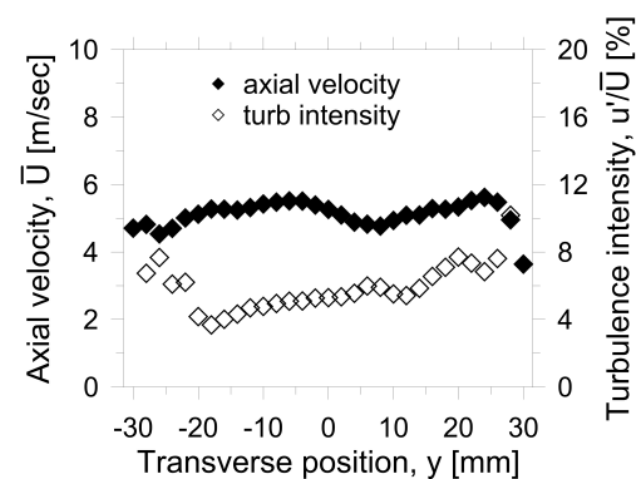

Figure 3.3 Transverse profiles of velocity $\diamond$ and turbulence intensity $\diamond$ in the burner exit plane at axial position $x=5 \mathrm{~mm}$.

\subsubsection{Reactant Characterization}

Equivalence ratio measurements were obtained through single line tracer PLIF by seeding iso-octane with 3-pentanone at $7 \%$ by volume to ensure the necessary signal to noise ratio [83], which ranged from 5.0-5.4 within the equivalence ratio range of interest (0.95-1.05) and 4.4-6.0 for $\phi=0.8-1.2$ (calculated as $\left(\mu_{\text {fluorescence }}-\mu_{\text {background }}\right) / \sigma_{\text {fluorescence }}[100]$, where $\mu_{\text {fluorescence }}$ and $\sigma_{\text {fluorescence }}$ are the mean and standard 
deviation of the fluorescence signal within a specified area in the reactants, $\mu_{\text {background }}$ is the mean background signal within an equivalent area of interest in the products). The choice of 3-pentanone ensured negligible effects of the locally varying mixture composition of the stratified flow field on the emitted fluorescence, since quenching of molecular oxygen is very weak for ketones at atmospheric pressure $[83,101,102]$. The incident laser fluence was kept below the saturation level of the tracer such that the fluorescence signal scales linearly with the local laser fluence and number density of the tracer $[84,103]$, while the use of a beam homogenizer further ensured minimal axial variation throughout the laser sheet. The temperature dependence of the fluorescence signal was significantly reduced by exciting the 3pentanone near its absorption maximum $(279.80 \mathrm{~nm}$ at $328 \mathrm{~K}$ [104]) at $282.75 \mathrm{~nm}$, such that tracer concentrations could be measured directly without corrections for temperature $[83,102]$.

One thousand flame images were acquired at each of three axial positions $\mathrm{x}=0-40,40-80$, and 80 $120 \mathrm{~mm}$ above the exit plane of the burner. The measurement frequency was limited to $<1 \mathrm{~Hz}$, ensuring each flame realization was statistically independent. Using analysis procedures similar to those described in [47], individual images were corrected to account for background intensity variation, non-uniform mean laser energy profile, and pulse to pulse variations in laser energy, before a $5 \times 5$ pixel median filter (equivalent to $0.34 \times 0.34 \mathrm{~mm}$ ) was applied. Fluorescence values were then related to equivalence ratio using a linear calibration scheme obtained by measuring fluorescence in non-reacting homogenous reactant mixtures of known equivalence ratio. Further details of this calibration are included as supplemental material. The resultant instantaneous equivalence ratio images were then masked to obtain conditioned images of the equivalence ratio in the reactants with a well defined edge on the upstream side of the flame. Binary masks were obtained starting from each raw, instantaneous fluorescence image, applying a blurring filter, a locally varying threshold, and finally an edge erosion as described in detail below.

To create the mask, first a circular averaging (or "pillbox") blurring filter with an 11 pixel diameter (equivalent to $0.74 \mathrm{~mm}$ ) was applied to the individual raw fluorescence images to smooth small scale fluctuations in fluorescence intensity. Given the relatively low signal to noise ratio in the very lean regions of the images (down to 2.7 at $\phi=0.4$ as compared to 5.0-5.4 in the $\phi=0.95-1.05$ region of interest), the application of a blurring filter was necessary to avoid removing regions of low fluorescence intensity (low pixel count caused by shot noise) when a threshold was subsequently applied. Next, a threshold was 
applied to the individual images, to isolate reactant regions (higher fluorescence intensity) and exclude product regions (negligible fluorescence intensity). In stratified cases, the large-scale gradients in equivalence ratio resulted in a transverse (y-direction) variation of the local pixel intensity within individual stratified images. To accommodate this variation, a locally varying threshold based on the mean equivalence ratio gradients previously determined for each flame setting was applied to the individual images. This approach was particularly effective in the most stratified cases, for which the equivalence ratio could vary from $\sim 0.2$ to 1.8 within an individual image, and allowed the application of a more aggressive threshold nearer to the leading edge of the reaction zone to more clearly resolve the location of the flame front. An example result, showing the application of this intermediate mask to an instantaneous fluorescence image, is shown in Figure 3.4a.

In Figure 3.4a there is a slight but noticeable decrease in the 3-pentanone fluorescence intensity along the leading edge (reaction front) of images that was typical of all instantaneous realizations. This decrease in fluorescence occurs within the preheat zone of the flame front, as confirmed via separate experiments in which 3-pentanone and $\mathrm{CH}_{2} \mathrm{O}$ were simultaneously acquired. As the temperature gradually increases across the reaction zone during the first reaction steps [101], the tracer signal decreases as the parent fuel breaks down [105]. This region therefore does not provide an accurate measure of equivalence ratio, and was discarded during image processing. Within the individual images, an erosion with a 7 pixel diameter (equivalent to $0.47 \mathrm{~mm}$ and roughly half the flame thickness) circular "disk" structuring element [106] was applied to obtain a final 3-pentanone PLIF image of the reactants, as seen in Figure 3.4b. The dimensions of the eroding element were selected to minimize the region where the 3-pentanone and $\mathrm{CH}_{2} \mathrm{O}$ signals overlapped, since $\mathrm{CH}_{2} \mathrm{O}$ is a good marker for the location of the initial temperature increase of the reactants. This ensured that the retained 3-pentanone fluorescence was not biased by potential temperature effects. As a result, the decrease in fluorescence signal perpendicular to the leading edge is much less apparent in Figure 3.4b than in Figure 3.4a, and this level of care in the image processing ensured that the remaining 3-pentanone signal, upstream of the pre-heat reaction zone, provided an accurate measure of equivalence ratio, which was critical to accurately determine the location of a relevant interrogation window, or region of interest (ROI), for comparisons among different gradient conditions as discussed below. 


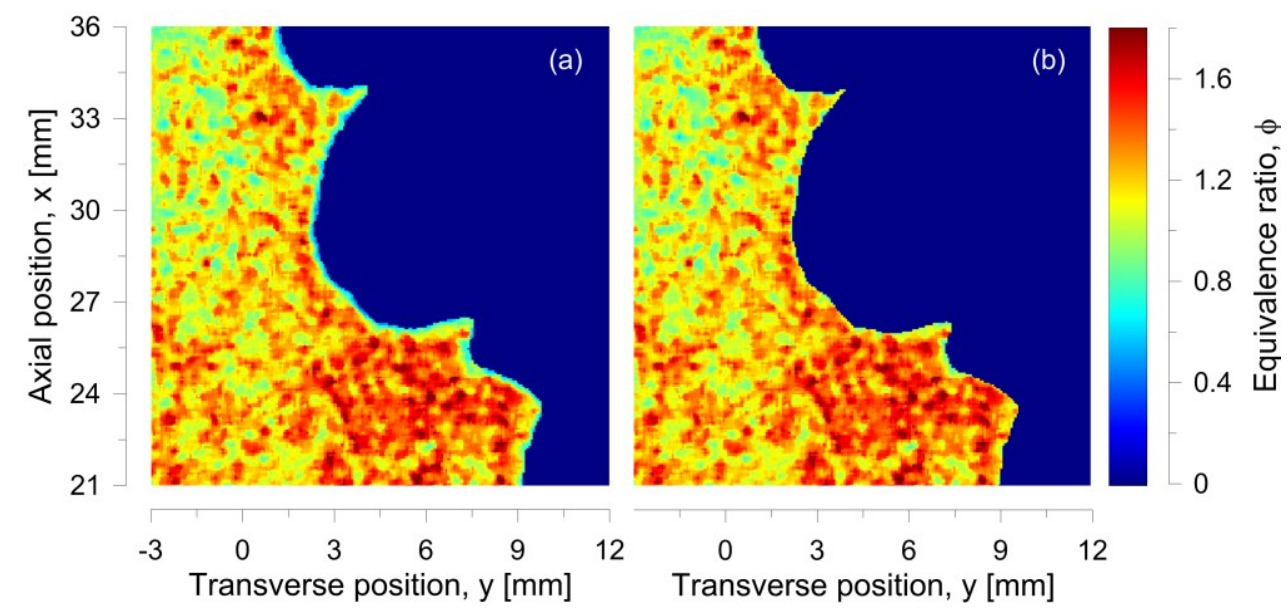

Figure 3.4 Close-up highlighting the decrease in signal intensity along leading edge of 3-pentanone front in (a) for non-eroded and in (b) for eroded image for stratified flame condition $\delta \phi / \delta y=0.021 \mathrm{~mm}^{-1}$.

Instantaneous equivalence ratio images obtained at each axial position were combined to provide equivalence ratio maps from $0 \mathrm{~mm} \leq \mathrm{x} \leq 120 \mathrm{~mm}$ above the exit plane of the burner. Each set of 1000 instantaneous images (a sample single image is shown in Figure 3.5a) were ensemble averaged and conditioned on the reactants to obtain the mean equivalence ratio within the turbulent flame brush. The mean position of the flame front, defined as $\langle c\rangle=0.5$, was determined from the ensemble average of the final binary masks corresponding to each of the 1000 instantaneous equivalence ratio images. Figure $3.5 \mathrm{~b}$ shows the final equivalence ratio map for stratified flame setting $\delta \phi / \delta y=0.021 \mathrm{~mm}^{-1}$ up to the mean flame contour. The $\phi=0.95$ and $\phi=1.05$ iso-surfaces were then identified, as highlighted in Figure $3.5 \mathrm{~b}$ for $\delta \phi / \delta \mathrm{y}=0.021 \mathrm{~mm}^{-1}$. From this, the dimensions of the ROI could be determined, which ranged from 3.5 to $12.5 \mathrm{~mm}$ depending on the equivalence ratio gradient. Thus, with this analysis approach, it was possible to define a specific ROI for each flame condition within which the range of mean equivalence ratios remained constant while the overall equivalence ratio gradient was experimentally varied. 


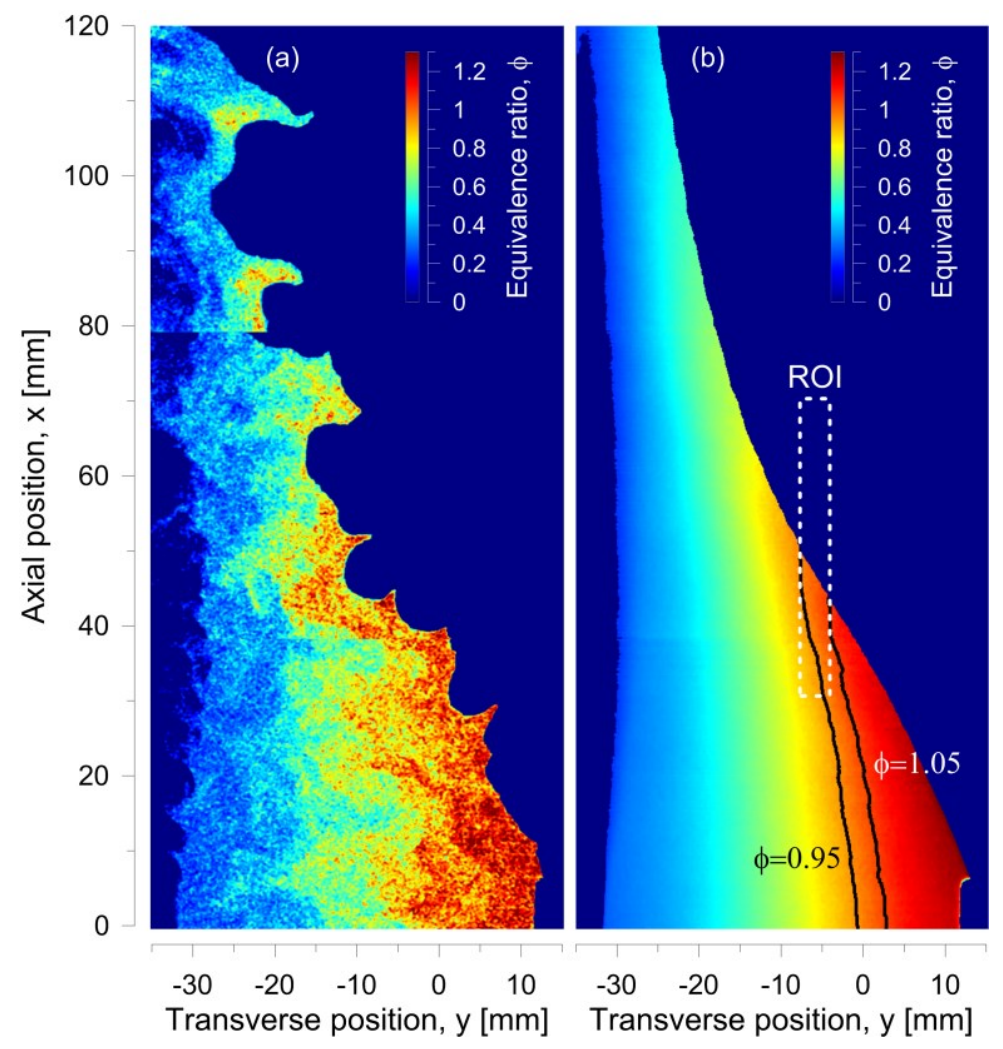

Figure 3.5 Example equivalence ratio contours derived from 3-pentanone fluorescence images for stratified flame setting $\delta \phi / \delta y=0.021 \mathrm{~mm}^{-1}$ showing (a) instantaneous and (b) average conditioned images.

Through analysis of the instantaneous 3-pentanone PLIF data, small-scale fluorescence intensity variations were observed on the order of $0.5-3 \mathrm{~mm}$, as shown in the sample image in Figure 3.5a. These small-scale local fluctuations were attributed primarily to shot noise, which ranged between $30.9 \%$ and $14.7 \%$ at $95 \%$ confidence for mean pixel counts corresponding to reactant equivalence ratios $\phi=0.4$ and $\phi=1.6$ respectively (determined from white field images acquired using a 4 " integrating sphere (SphereOptics LLC) and calculated using photon transfer theory [107]). This implies that discussion of local instantaneous variations in equivalence ratio should be limited to larger structures, on the order of roughly $10 \mathrm{~mm}$, which were attributed to the turbulent mixing of the large scale gradients in equivalence ratio imposed by the burner. 
Table 3.1 Test conditions and reactant properties.

\begin{tabular}{|c|c|c|c|c|c|c|c|c|c|}
\hline & & & \multicolumn{4}{|c|}{ Windowed analysis ROI } & \multicolumn{3}{|c|}{ Non-Windowed analysis ROI } \\
\hline $\begin{array}{c}\text { iso- } \\
\text { contours } \\
\text { of } \phi \\
\text { defining } \\
\text { the ROI }\end{array}$ & $\begin{array}{c}\text { equivalence } \\
\text { ratio gradient, } \\
\delta \phi / \delta \mathrm{y} \\
{\left[\phi \mathrm{mm}^{-1}\right]}\end{array}$ & $\begin{array}{c}\text { turbulent } \\
\text { flame } \\
\text { brush } \\
\text { thickness, } \\
\mathrm{L}_{\mathrm{t}, \text { strat }} / \mathrm{L}_{\mathrm{t}, \mathrm{ref}} \\
{[\mathrm{mm}]}\end{array}$ & $\begin{array}{l}\text { width } \\
\text { of } \\
\text { ROI } \\
{[\mathrm{mm}]}\end{array}$ & $\begin{array}{c}\text { mean } \phi \text { of } \\
\text { instantaneous } \\
\text { images } \\
\text { within } \\
0.95 \leq \phi \leq 1 \\
05 \text { iso- } \\
\text { contours and } \\
\text { estimated } \\
\text { standard } \\
\text { deviation of } \\
\phi\end{array}$ & $\begin{array}{c}\text { mean and } \\
\text { estimated } \\
\text { standard } \\
\text { deviation of } \\
\phi \text { along } \\
\phi=0.95 \text { iso- } \\
\text { contour in } \\
\text { instantaneou } \\
\text { s images }\end{array}$ & $\begin{array}{c}\text { mean and } \\
\text { estimated } \\
\text { standard } \\
\text { deviation of } \\
\phi \text { along } \\
\phi=1.05 \text { iso- } \\
\text { contour in } \\
\text { instantaneou } \\
\text { s images }\end{array}$ & $\begin{array}{c}\text { mean } \phi \text { of } \\
\text { instantaneous } \\
\text { images } \\
\text { within lean } \\
\text { and rich iso- } \\
\text { contours and } \\
\text { estimated } \\
\text { standard } \\
\text { deviation of } \\
\phi\end{array}$ & $\begin{array}{c}\text { mean and } \\
\text { estimated } \\
\text { standard } \\
\text { deviation of } \\
\phi \text { along lean } \\
\text { iso-contour } \\
\text { in } \\
\text { instantaneo } \\
\text { us images }\end{array}$ & $\begin{array}{c}\text { mean and } \\
\text { estimated } \\
\text { standard } \\
\text { deviation of } \\
\phi \text { along rich } \\
\text { iso-contour } \\
\text { in } \\
\text { instantaneo } \\
\text { us images }\end{array}$ \\
\hline \multirow{2}{*}{$\begin{array}{c}0.95 \\
\pm \\
0.022\end{array}$} & -0.001 & - & 12.5 & $1.012(-)$ & - & - & $1.018(-)$ & - & - \\
\hline & 0.008 & 1.01 & 12.5 & $\begin{array}{c}0.999 \\
(0.008)\end{array}$ & $\begin{array}{c}0.951 \\
(0.029)\end{array}$ & $\begin{array}{c}1.050 \\
(0.031)\end{array}$ & $\begin{array}{c}1.001 \\
(0.006)\end{array}$ & $\begin{array}{c}0.921 \\
(0.028)\end{array}$ & $\begin{array}{c}1.089 \\
(0.037)\end{array}$ \\
\hline \multirow{3}{*}{$\begin{array}{c}1.05 \\
\pm \\
0.023\end{array}$} & 0.015 & 1.04 & 6.7 & $\begin{array}{c}1.000 \\
(0.018)\end{array}$ & $\begin{array}{c}0.949 \\
(0.043)\end{array}$ & $\begin{array}{c}1.049 \\
(0.044)\end{array}$ & $\begin{array}{c}1.003 \\
(0.007)\end{array}$ & $\begin{array}{c}0.870 \\
(0.042)\end{array}$ & $\begin{array}{c}1.137 \\
(0.052)\end{array}$ \\
\hline & 0.021 & 1.09 & 4.8 & $\begin{array}{c}1.000 \\
(0.024)\end{array}$ & $\begin{array}{c}0.950 \\
(0.061)\end{array}$ & $\begin{array}{c}1.051 \\
(0.061)\end{array}$ & $\begin{array}{c}0.984 \\
(0.018)\end{array}$ & $\begin{array}{c}0.767 \\
(0.061)\end{array}$ & $\begin{array}{c}1.177 \\
(0.066)\end{array}$ \\
\hline & 0.029 & 1.11 & 3.5 & $\begin{array}{c}1.001 \\
(0.029)\end{array}$ & $\begin{array}{c}0.952 \\
(0.081)\end{array}$ & $\begin{array}{c}1.051 \\
(0.080)\end{array}$ & $\begin{array}{c}0.981 \\
(0.022)\end{array}$ & $\begin{array}{c}0.737 \\
(0.072)\end{array}$ & $\begin{array}{c}1.218 \\
(0.090)\end{array}$ \\
\hline
\end{tabular}

${ }^{*}$ The reference turbulent flame brush thickness, $\mathrm{L}_{\mathrm{t}, \mathrm{ref}}$, measured at the same axial location as the corresponding stratified case, was 7.3 , $7.0,7.0$, and $6.8 \mathrm{~mm}$ for the weakest to the steepest gradient case.

Table 3.1 also shows reactant properties for non-windowed data (i.e. ROI comprising the entire image), used to evaluate the general effect of stratification on the overall heat release rate. For these experiments, global stoichiometry was conserved within $\phi= \pm 0.02$ for all flame conditions, while the range of equivalence ratios increases as the gradient steepened. Individual images were evaluated between the same axial positions $\mathrm{x}=34.2 \mathrm{~mm}$ to $\mathrm{x}=69.4 \mathrm{~mm}$ for all flame conditions.

\subsubsection{Equivalent ROI Approach to Quantifying Mean Equivalence Ratio Gradient Effects in a Turbulent Flow Field}

For each mean gradient setting, an ROI was defined that was bounded by the mean $\phi=0.95$ and $\phi=1.05$ iso-contours obtained from 3-pentanone tracer PLIF images of the reactants. As outlined in Table 3.1, while this ensured that the mean range of equivalence ratios within each ROI was constant, as the gradient of $\phi$ was increased it was expected that instantaneous variations of $\phi$ within the ROI would similarly broaden. The standard deviation of $\phi$ within each ROI $\left(\sigma_{\phi}\right)$ was characterized as the square root of the difference between the variance attributable to measurement noise (characterized as the statistical variance at equivalent spatial locations for the fully premixed reference case, $\sigma_{\text {Ref,premixed }}^{2}$ ) and the observed 
variance in the raw fluorescence data $\left(\sigma_{\mathrm{PLIF}}^{2}\right)$ for each of the stratified cases (i.e. $\left.\sigma_{\phi}=\sqrt{\sigma_{\text {PLIF }}^{2}-\sigma_{\text {Ref,premixed }}^{2}}\right)$. The resulting estimates of $\sigma_{\phi}$ are representative of the variations in equivalence ratio due to turbulent mixing, and are presented in Table 1. Results show that $\sigma_{\phi}$ within the ROI grows modestly as the gradient increases, ranging from 0.029 to 0.081 and 0.031 to 0.080 along the $\phi=0.95$ and 1.05 iso-contours respectively. In the context of results presented in Section 3.4 potential effects of this increased variation of $\phi$ within the ROI on the measured mean heat release rate were assessed in a Monte Carlo approach by generating synthetic distributions of $\phi$, where the effects of turbulent mixing were approximated based on the standard deviations of $\phi$ along iso-contours between $\phi=0.95$ and $\phi=1.05$ in steps of $\phi=0.01$. Twenty thousand instantaneous $\phi$ distributions were generated, and for each a mean heat release rate was calculated based on simulated heat release rate data for premixed flames (details of the simulations are discussed in Section 3.3.2.1). Results showed that the increased variation of $\phi$ within the fixed range ROI leads to a maximum decrease in mean $\mathrm{HRR}$ of $1.6,2.3,3.5$, or $5.3 \%$ relative to the reference premixed case, as the gradient is increased. As further discussed in Section 3.4.2, in terms of the measured product of $\mathrm{OH}$ and $\mathrm{CH}_{2} \mathrm{O}$ fluorescence, a slightly smaller maximum decrease of $0.9-1.0 \%$ (depending on the choice of $\mathrm{CH}_{2} \mathrm{O}$ quenching model) to $4.1-4.3 \%$ would be expected for the weakest to steepest gradient cases respectively.

\subsection{2 $\mathrm{OH}$ and $\mathrm{CH}_{2} \mathrm{O}$ PLIF Images}

For each of the five flame conditions, 1000 individual $\mathrm{OH}$ and $\mathrm{CH}_{2} \mathrm{O}$ PLIF images were acquired. A 225 point target was used to register the $\mathrm{CH}_{2} \mathrm{O}$ and $\mathrm{OH}$ images to ensure precise spatial correspondence of the images recorded by each camera. A linear conformal correlation, which preserves angles and shapes of the input image, was used with a bilinear interpolation to map control points on the common target. A correlation function was determined immediately prior to running experiments to ensure accurate spatial registration between $\mathrm{OH}$ and $\mathrm{CH}_{2} \mathrm{O}$ images, and final data were acquired in rapid succession for each of the premixed and gradient cases.

Acquired $\mathrm{OH}$ and $\mathrm{CH}_{2} \mathrm{O}$ PLIF images were corrected for background intensity as well as laser profile and pulse to pulse energy fluctuations, in the same manner as the 3-pentanone images. Individual $\mathrm{OH}$ images were filtered using a $3 \times 3$ pixel median filter (equivalent to a physical dimension of 
$0.20 \times 0.20 \mathrm{~mm}$ ) to reduce shot noise. The $\mathrm{CH}_{2} \mathrm{O}$ images were filtered with a 7 pixel circular averaging filter (equivalent to $0.47 \mathrm{~mm}$ in diameter) because of their relatively lower signal to noise ratio. Regions of $\mathrm{OH}$ and $\mathrm{CH}_{2} \mathrm{O}$ were identified by masking the individual fluorescence images to retain signals above a chosen threshold. Images were evaluated to quantify the effect of stratification on $\mathrm{OH}$ and $\mathrm{CH}_{2} \mathrm{O}$ radical fluorescence, and heat release rate.

Instantaneous heat release rate images were obtained by multiplying the spatially registered, simultaneously acquired greyscale $\mathrm{OH}$ and $\mathrm{CH}_{2} \mathrm{O}$ PLIF images. This approach, proposed by Paul and Najm [55], is based on the temporal and spatial correlation between the concentration of formyl radical [HCO] and the heat release rate $[108,109]$. They concluded that a weak signal-to-noise ratio $(2: 1)$ was insufficient to successfully apply HCO PLIF to turbulent reacting flows [109], but that an alternative method can be derived from the intermediate forward reaction,

$$
\mathrm{OH}+\mathrm{CH}_{2} \mathrm{O} \rightarrow \mathrm{HCO}+\mathrm{H}_{2} \mathrm{O}
$$

whereby the product of $[\mathrm{OH}]$ and $\left[\mathrm{CH}_{2} \mathrm{O}\right]$ can be used as a direct measure of $[\mathrm{HCO}]$ and therefore the heat release rate [55]. Contrary to other experimental methods such as chemiluminescence or the dilatation rate, the approach proposed by [55] is a spatially resolved, direct chemical measurement of the reaction rate, and remains a valid approximation of the reaction rate even in regions of high strain or where local extinctions may occur. This approach to evaluating heat release rate is based on the scaling of the forward reaction rate $\mathrm{k}(\mathrm{T})[\mathrm{OH}]\left[\mathrm{CH}_{2} \mathrm{O}\right]$, where $\mathrm{k}(\mathrm{T})$ is the forward rate constant, with the product of the fluorescence signals $(\mathrm{OH} \mathrm{LIF})\left(\mathrm{CH}_{2} \mathrm{O}\right.$ LIF). The fluorescence signals can be estimated from $\mathrm{f}_{\mathrm{OH}}(\mathrm{T})[\mathrm{OH}]$ and $\mathrm{f}_{\mathrm{CH}_{2} \mathrm{O}}(\mathrm{T})\left[\mathrm{CH}_{2} \mathrm{O}\right]$, where $f(T)$ represents the temperature dependence of the fluorescent signal, which is specific to the excitation transition being used and is a function of the ground state Boltzmann population fraction as well as the quenching cross section. Accurate heat release rate measurements therefore require that $\mathrm{f}_{\mathrm{OH}}(\mathrm{T}) \mathrm{f}_{\mathrm{CH}_{2} \mathrm{O}}(\mathrm{T}) \propto \mathrm{k}(\mathrm{T})$.

\subsubsection{Validation of (OH LIF)( $\mathrm{CH}_{2} \mathrm{O}$ LIF) for Heat Release Rate Measurements in Stratified Iso-Octane/Air Flames}

While the measurement of heat release rate from the product of $\mathrm{OH}$ and $\mathrm{CH}_{2} \mathrm{O}$ fluorescence has been successfully applied to simple hydrocarbon fuels such as methane [55], ethylene [57], and propane [56], it 
has yet to be validated for iso-octane/air flames. Detailed numerical calculations of one-dimensional laminar premixed iso-octane/air flames were conducted at equivalence ratios ranging from $\phi=0.75-1.25$, using a code developed by Kee et al. [110]. Upwind and center difference schemes were used for the convective and diffusion terms, respectively, in all the governing equations, and adaptive mesh refinement was implemented. The pressure and the reactant mixture temperature were $1 \mathrm{~atm}$ and $328 \mathrm{~K}$, and matched those of the current experiments. The iso-octane oxidation kinetics mechanism developed in [111] was used. The thermal and transport properties were obtained by using the algorithms given in $[112,113]$. Simulations explicitly considered thermal diffusive velocities of $\mathrm{H}_{2}$ and $\mathrm{H}$, while those of other species were deemed negligible.

Figure 3.6 shows the normalized profiles of $[\mathrm{OH}],\left[\mathrm{CH}_{2} \mathrm{O}\right],[\mathrm{HCO}],[\mathrm{OH}]\left[\mathrm{CH}_{2} \mathrm{O}\right]$, temperature, and heat release rate for simulated flame conditions $\phi=0.75, \phi=1.00$, and $\phi=1.25$. The temperature profiles tend towards adiabatic values at a downstream position in the products, beyond the limits of the plots. The alignment of the product of the $[\mathrm{OH}]$ and $\left[\mathrm{CH}_{2} \mathrm{O}\right]$ profiles with the $[\mathrm{HCO}]$ and $\mathrm{HRR}$ profiles is remarkably consistent with those reported by Paul and Najm [55] for premixed methane/air flames. The measured peak-to-peak distance of the $[\mathrm{HCO}]$ and $[\mathrm{OH}]\left[\mathrm{CH}_{2} \mathrm{O}\right]$ profiles with the heat release rate ranged between 3-19 $\mu \mathrm{m}$ and 13-32 $\mu \mathrm{m}$ for all flame settings respectively, confirming their spatial agreement for a broad range of premixed iso-octane/air flames.

The temperature dependence of the fluorescence signals was estimated based on the results of the simulations. The current experiments probed the $\mathrm{Q}_{1}(6)$ transition for $\mathrm{OH}$ similar to $[55-57,60-62,114]$ and the $\widetilde{\mathrm{A}}^{1} \mathrm{~A}_{2}-\widetilde{\mathrm{X}}^{1} \mathrm{~A}_{1} 4_{0}^{1}$ vibronic manifold for $\mathrm{CH}_{2} \mathrm{O}$ similar to $[56,60-62,93,115,116]$. The Boltzmann population fractions and collisional quenching rate for $\mathrm{OH}$ have been well characterized and were determined from [117] and [118] respectively. The analysis of Clouthier and Ramsay [94,119] was followed to determine the population fractions of $\mathrm{CH}_{2} \mathrm{O}$. Since there is currently no model for $\mathrm{CH}_{2} \mathrm{O}$ quenching that accounts for specific collisional partners, following [55] this was approximated based on the temperature dependence of the quenching cross section $\sigma$. Two limiting models were considered: the first assumed a constant quenching cross section, resulting in a quenching rate of $\mathrm{Q}_{21} \sim \mathrm{T}^{-0.5}$, while the second assumed a $\sigma \sim \mathrm{T}^{-0.5}$ variation over the range of temperatures encountered, resulting in a quenching rate of 
$\mathrm{Q}_{21} \sim \mathrm{T}^{-1}[55,94]$. For the $\mathrm{OH}+\mathrm{CH}_{2} \mathrm{O} \rightarrow \mathrm{HCO}+\mathrm{H}_{2} \mathrm{O}$ reaction, the forward rate constant is $\mathrm{k}(\mathrm{T}) \propto \mathrm{T}^{0.89} \exp (-204.5 / \mathrm{T})[120]$.

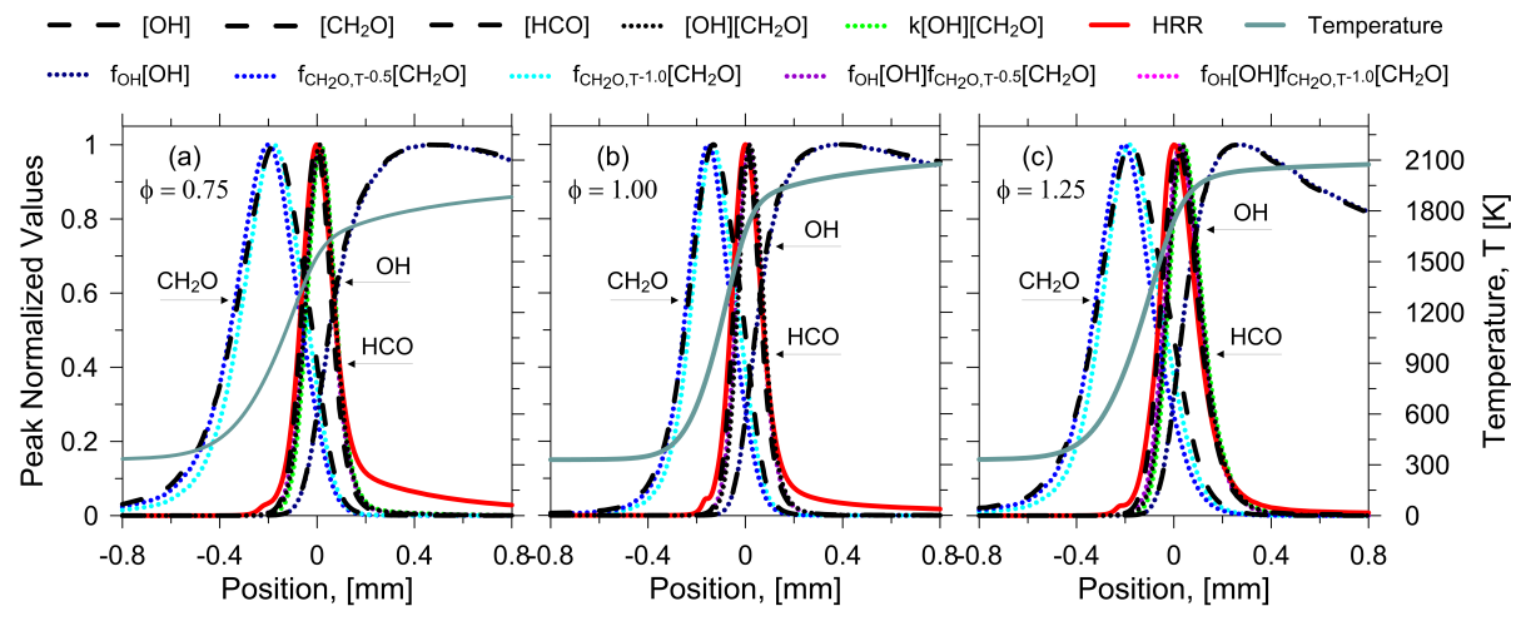

Figure 3.6 Normalized profiles of [OH], $\left[\mathrm{CH}_{2} \mathrm{O}\right]$, [HCO], [OH][CH2O], $\mathrm{k}[\mathrm{OH}]\left[\mathrm{CH}_{2} \mathrm{O}\right]$, $\mathrm{HRR}$,

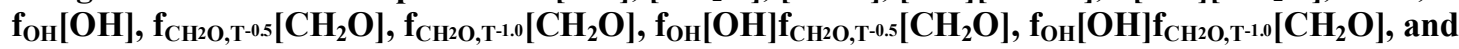
Temperature for (a) $\phi=0.75$, (b) $\phi=1.00$, and (c) $\phi=1.25$.

The temperature dependence of the $\mathrm{f}_{\mathrm{OH}}(\mathrm{T})[\mathrm{OH}], \mathrm{f}_{\mathrm{CH}_{2} \mathrm{O}}(\mathrm{T})\left[\mathrm{CH}_{2} \mathrm{O}\right]$, and corresponding $\left(\mathrm{OH}\right.$ LIF) $\left(\mathrm{CH}_{2} \mathrm{O}\right.$ LIF) fluorescence signals $\left(=\mathrm{f}(\mathrm{T})[\mathrm{OH}]\left[\mathrm{CH}_{2} \mathrm{O}\right]\right)$ are also plotted in Figure 3.6. The $\mathrm{OH}$ profiles showed negligible variation between $\mathrm{f}_{\mathrm{OH}}(\mathrm{T})[\mathrm{OH}]$ and $[\mathrm{OH}]$ for lean, stoichiometric, and rich flames, which is expected based on the choice of the relatively temperature insensitive $Q_{1}(6)$ transition for the current experiments. The $\mathrm{CH}_{2} \mathrm{O}$ profiles showed a small bias towards the reactants for the $\mathrm{T}^{-0.5}$ quenching model, while the bias for the $\mathrm{T}^{-1.0}$ model was less significant. The net effect on the $\mathrm{f}(\mathrm{T})[\mathrm{OH}]\left[\mathrm{CH}_{2} \mathrm{O}\right]$ fluorescence profile remained small, implying that the product of $\mathrm{OH}$ and $\mathrm{CH}_{2} \mathrm{O}$ fluorescence is primarily dependent on the $[\mathrm{OH}]$ and $\left[\mathrm{CH}_{2} \mathrm{O}\right]$ concentrations, and relatively insensitive to $f(T)$, consistent with the observations of Ayoola et al [57] for lean ethylene/air flames. Comparing $\mathrm{f}(\mathrm{T})[\mathrm{OH}]\left[\mathrm{CH}_{2} \mathrm{O}\right]$ with the forward reaction rate $\mathrm{k}(\mathrm{T})[\mathrm{OH}]\left[\mathrm{CH}_{2} \mathrm{O}\right]$ profile further validated the approach, as the peak-to-peak distance ranged between $6-13 \mu \mathrm{m}$ and $10-13 \mu \mathrm{m}$ from $\phi=0.75-1.25$, for the $\mathrm{T}^{-1}$ and $\mathrm{T}^{-0.5}$ $\mathrm{CH}_{2} \mathrm{O}$ models respectively, confirming that the (OH LIF) $\left(\mathrm{CH}_{2} \mathrm{O}\right.$ LIF) approach to measurements of heat release rate is applicable to premixed iso-octane/air flames over a broad range of $\phi$. 
The applicability of the approach to stratified mixtures was further evaluated by plotting $(\mathrm{OH}$ LIF $)\left(\mathrm{CH}_{2} \mathrm{O}\right.$ LIF) as a function of both $\mathrm{k}(\mathrm{T})[\mathrm{OH}]\left[\mathrm{CH}_{2} \mathrm{O}\right]$ and $\mathrm{HRR}$, for the range of simulated $\phi$. While the correlation between $(\mathrm{OH} \mathrm{LIF})\left(\mathrm{CH}_{2} \mathrm{O} \mathrm{LIF}\right)$ and $\mathrm{k}(\mathrm{T})[\mathrm{OH}]\left[\mathrm{CH}_{2} \mathrm{O}\right]$ is of interest (and is included in Section 3.6.2), the object of the current study is to characterize the direct correlation between fluorescence signals and heat release rate. The $\left(\mathrm{OH}\right.$ LIF) $\left(\mathrm{CH}_{2} \mathrm{O}\right.$ LIF) and $\mathrm{HRR}$ data were normalized by the peak value of the $\phi=1.00$ profiles, where Figure 3.7a and Figure 3.7b show the same profiles calculated using the $\mathrm{CH}_{2} \mathrm{O}$ quenching models $\mathrm{T}^{-0.5}$ or $\mathrm{T}^{-1.0}$ respectively.

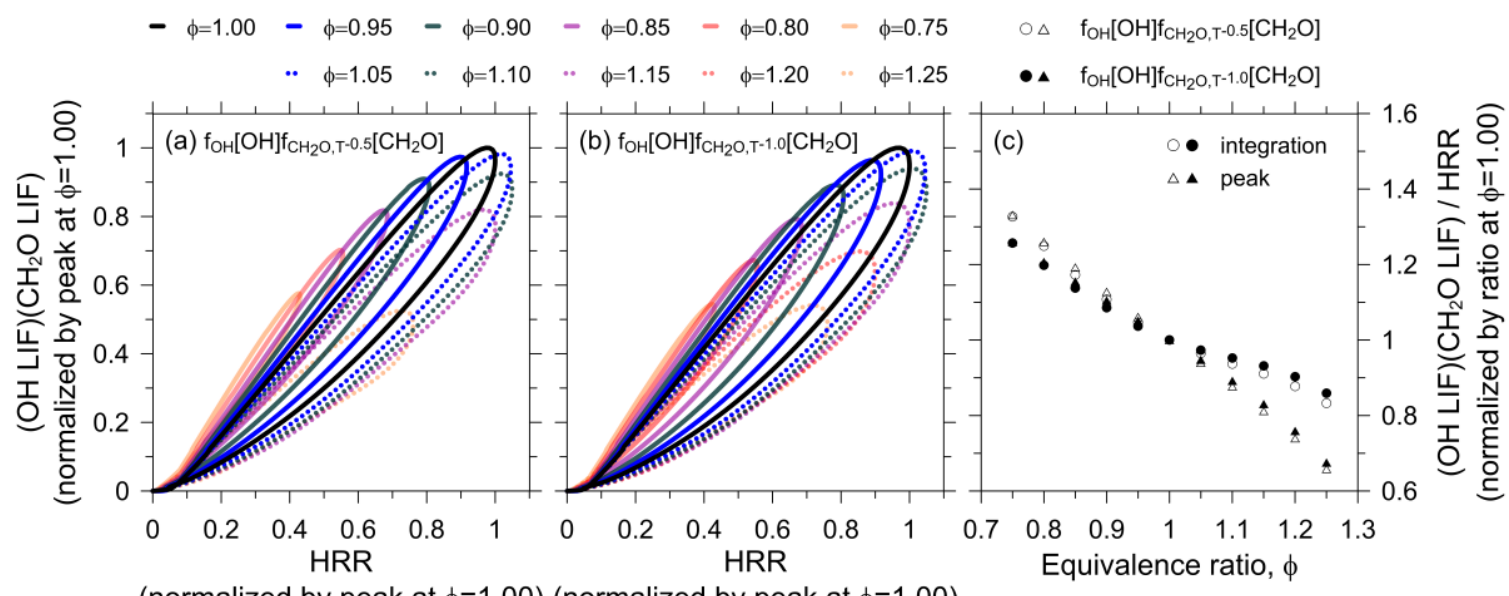

(normalized by peak at $\phi=1.00$ ) (normalized by peak at $\phi=1.00$ )

Figure 3.7 Correlation of (OH LIF)(CH $\left(\mathrm{CH}_{2} \mathrm{O} \mathrm{LIF}\right)$ with heat release rate for $\mathrm{CH}_{2} \mathrm{O}$ quenching models $T^{-0.5}$ in (a) and $T^{-1.0}$ in (b) for simulated premixed flames ranging from $\phi=0.75-1.25$. Variation of the ratio of integrated and peak (OH LIF) $\left(\mathrm{CH}_{2} \mathrm{O} \mathrm{LIF}\right) / \mathrm{HRR}$ over a range of equivalence ratios is presented in (c).

The loops in both Figure 3.7a and Figure 3.7b represent the variation of temperature induced bias errors through the flame, from the reactant side (lower part of the loop) to the product side (upper part of the loop) of the individual premixed cases. The plots show good agreement between premixed conditions $\phi=0.95-1.00-1.05$, as the corresponding profiles closely align. As flames become richer/leaner, the correlation between $(\mathrm{OH} \mathrm{LIF})\left(\mathrm{CH}_{2} \mathrm{O} \mathrm{LIF}\right)$ and the $\mathrm{HRR}$ diverges relative to the reference premixed case, as Boltzmann fractions and quenching rates vary more significantly relative to the $\phi=1.00$ condition. The potential bias from these effects is summarized in Figure 3.7c, which plots the ratio of the integral $(\mathrm{OH} \mathrm{LIF})\left(\mathrm{CH}_{2} \mathrm{O} \mathrm{LIF}\right) / \mathrm{HRR}$, normalized by the ratio of the reference premixed case at $\phi=1.00$ (ratios for the peak $(\mathrm{OH} \mathrm{LIF})\left(\mathrm{CH}_{2} \mathrm{O} \mathrm{LIF}\right) / \mathrm{HRR}$ are also included). The ratios gradually deviate from 1 as $\phi$ is varied, 
with bias errors of the integrated signals between $+3.7 \%$ and $+4.6 \%$ at $\phi=0.95$ and $-2.7 \%$ and $-3.5 \%$ at $\phi=$ 1.05 for the $\mathrm{T}^{-1}$ and $\mathrm{T}^{-0.5} \mathrm{CH}_{2} \mathrm{O}$ quenching models respectively. Thus, within a relatively narrow range of equivalence ratios, the (OH LIF) $\left(\mathrm{CH}_{2} \mathrm{O}\right.$ LIF) signal correlates directly to HRR. This suggests that local HRR measurements are feasible without the need for temperature corrections if the local equivalence ratio does not vary significantly.

Although Figure 3.7c suggests larger variations will occur over a broader range of equivalence ratios, the near-linear trend also shows that the increasing bias error for lean flames is offset by the decreasing bias error for rich flames. This implies that while heat release rate measurements within highly stratified mixtures would require temperature corrections to be able to compare local variations along a flame front, for an ROI with a symmetric distribution of $\phi$, measurements of the mean (OH LIF)( $\mathrm{CH}_{2} \mathrm{O}$ LIF) signal will still adequately represent the overall HRR. Based on Monte Carlo simulations described in Section 3.3.1.1, the effect of temperature on the measured fluorescence signals would lead to a negligible overestimation of the mean HRR within the ROI of $\sim 0.7-0.9 \%, 0.9-1.1 \%, 1.2-$ $1.5 \%$, and $1.6-1.9 \%$ for the weakest to the strongest gradient case, where the range in each case stems from the bounding $\mathrm{T}^{-1}$ and $\mathrm{T}^{-0.5} \mathrm{CH}_{2} \mathrm{O}$ quenching models. Coupled with the expected decrease in mean HRR due to the broader range of $\phi$ within the ROI detailed in Section 3.3.1.1, these offsetting effects would lead to an overall net decrease in mean $(\mathrm{OH} \mathrm{LIF})\left(\mathrm{CH}_{2} \mathrm{O}\right.$ LIF) HRR within the ROI ranging from $0.9-1.0 \%$, 1.4$1.6 \%, 2.5-2.7 \%$, and $4.1-4.3 \%$ for the weakest to steepest gradient cases.

Additional contributions to the uncertainty of comparative heat release rate measurements such as variations in laser absorption, line shape, and fluorescence trapping are expected to vary negligibly for flame regions within the ROI among gradient settings. Though shot-to-shot variations in local laser fluence profile would have occurred, they should induce linear variations in local fluorescence, and therefore average out over the 1000 image pairs considered for each gradient case. Long-term drift in laser intensity that might occur over an entire test run was considered by correcting individual $\mathrm{OH}$ and $\mathrm{CH}_{2} \mathrm{O}$ PLIF images for pulse-to-pulse variations. 


\subsubsection{Determination of the Location of the Flame Front and Turbulent Flame Brush Thickness}

The position of the flame front was precisely defined to coincide with the leading edge of the instantaneous heat release rate contours. For flame realizations in which the heat release rate contour was continuous throughout the image, the position of the flame front was identified by simple thresholding of the individual HRR images. The resulting binary image was then segmented, or subdivided, into reactants (pixel value of zero) and products (pixel value of one). Alternatively, for flame images in which the leading heat release rate contour was not continuous, a second reference edge was required to clearly delimit products from reactants. Figure $3.8 \mathrm{c}$ shows the combination of individual corresponding binary $\mathrm{OH}$ (Figure 3.8a) and $\mathrm{CH}_{2} \mathrm{O}$ (Figure 3.8b) images, and highlights the presence of such discontinuities along the corrugated flame front for stratified flame setting $\delta \phi / \delta y=0.029 \mathrm{~mm}^{-1}$. The red pixels in the figure represent the overlap of both species, and inherently the location of the instantaneous HRR contour. Although no measureable $\mathrm{OH}$ was present at the location of the discontinuities, the $\mathrm{CH}_{2} \mathrm{O}$ profiles remained continuous in all flame images. Therefore, the edge on the products side of the $\mathrm{CH}_{2} \mathrm{O}$ contour, which corresponds to the end of the "cool flame" region of the reaction zone, was deemed a good secondary flame-front marker. A continuous edge was thus obtained by subtracting the overlapping pixels between the binary $\mathrm{OH}$ and $\mathrm{CH}_{2} \mathrm{O}$ images (i.e. the red pixels in Figure 3.8c) from the binary $\mathrm{CH}_{2} \mathrm{O}$ contour (i.e. the green pixels in Figure $3.8 \mathrm{~b}$ and c), clearly delimiting products from reactants. Reactant and product regions in the resulting binary image were identified as previously described. A sample final instantaneous binary image is shown in Figure 3.8d.

Figure 3.8a also highlights the presence of edges within the products (in addition to the discontinuities along the flame front) which are an artefact of thresholding, as fluorescence intensity significantly decreases downstream of the flame front for severely stratified flame conditions. Under these conditions it would be very difficult to use $\mathrm{OH}$ signals on their own to reliably identify flame front regions, especially for the strongest gradient cases, as is apparent in Figure 3.9. The alternative combined $\mathrm{CH}_{2} \mathrm{O} / \mathrm{OH}$ approach used here ensured an accurate resolution of the position of the flame front, clearly demarking the reactants from the products, which was critical to an accurate representation of the mean reaction progress variable $\langle c\rangle$, obtained by ensemble averaging the final instantaneous binary images. 


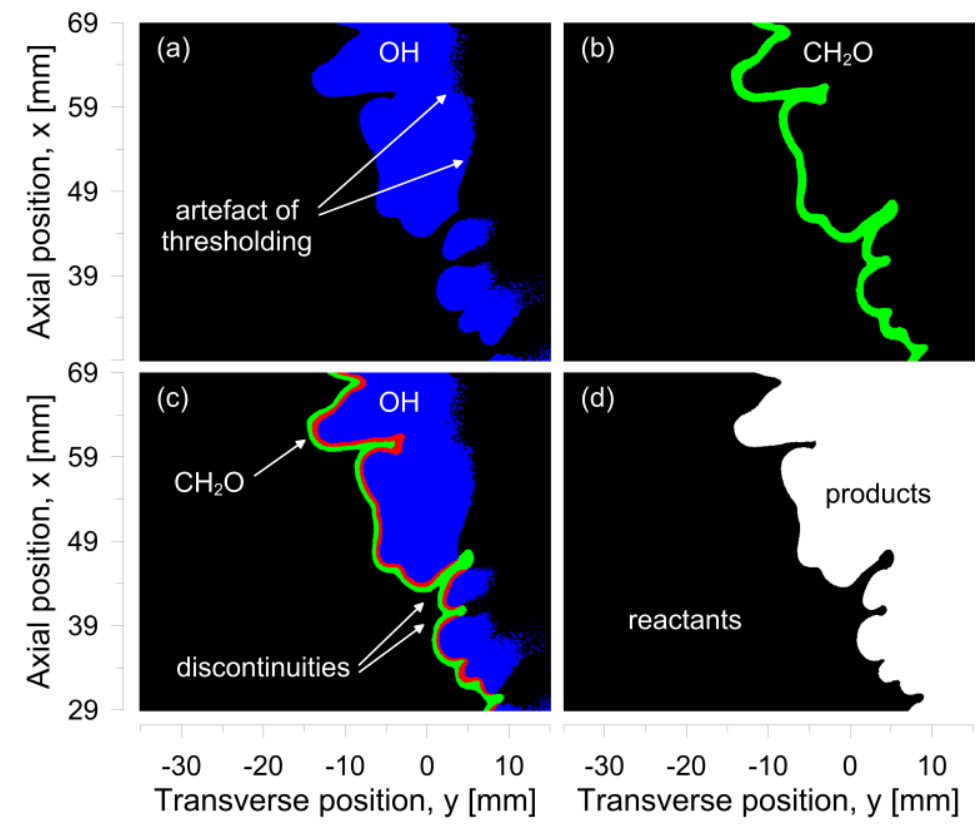

Figure 3.8 Instantaneous flame front position from heat release rate contour and product side of $\mathrm{CH}_{2} \mathrm{O}$ contour by image segmentation for stratified flame condition $\partial \phi / \partial y=0.029 \mathrm{~mm}^{-1}$.

The turbulent flame brush thickness $\mathrm{L}_{\mathrm{t}}$ was measured at the intersection of the reactant $\langle\mathrm{c}\rangle=0.5$ and $\phi=1.0$ iso-contours. Local values of $\langle\mathrm{c}\rangle$ were extracted using bicubic interpolation along the mean flame normal, defined relative to the $\langle\mathrm{c}\rangle=0.5$ contour. $\mathrm{L}_{\mathrm{t}}$ was then calculated by fitting a Gaussian profile to the extracted $\langle\mathrm{c}\rangle$ data, and determining the distance between $\langle\mathrm{c}\rangle=0.1$ and $\langle\mathrm{c}\rangle=0.9$ iso-contours. This methodology was applied to all gradient settings and results are expressed in Table 3.1 as the ratio $\mathrm{L}_{\mathrm{t}, \text { strat }} / \mathrm{L}_{\mathrm{t}, \text { refe }}$, where $\mathrm{L}_{\mathrm{t}, \text { ref }}$ is the brush thickness of the reference premixed case measured at the same axial location as the corresponding stratified case. The brush thickness $\mathrm{L}_{\mathrm{t}, \text { ref }}$ ranged from 6.8 to $7.3 \mathrm{~mm}$.

\subsection{Results and Discussion}

\subsubsection{Instantaneous $\mathrm{OH}, \mathrm{CH}_{2} \mathrm{O}$, and Heat Release Rate PLIF Images}

Figure 3.9 provides a false colour representation of a typical set of instantaneous $\mathrm{OH}, \mathrm{CH}_{2} \mathrm{O}$, and corresponding heat release rate images for each flame setting, ranging from fully-premixed to severely stratified. At a projected spatial resolution of $67 \mu \mathrm{m} / \mathrm{pixel}$, the images represent a $48.3 \mathrm{~mm} \times 40.0 \mathrm{~mm}$ region of interest within the flame. The mean signal to noise ratio of the individual OH PLIF images was 14.3, calculated following the same approach as for the 3-pentanone PLIF images (i.e. SNR $=\left(\mu_{\text {fluorescence }}\right.$ - 
$\left.\left.\mu_{\text {background }}\right) / \sigma_{\text {fluorescence }}\right)$. The signal to noise ratio of the $\mathrm{CH}_{2} \mathrm{O}$ PLIF data was similarly calculated taking into account the Gaussian-like signal variation through the flame, such that $\mathrm{SNR}=\left(\mu_{\mathrm{fluorescence}}\right.$, peak $\left.\mu_{\text {background }}\right) / \sigma_{\text {fluorescence,peak, }}$ with a mean value of 5.4.

$\mathrm{OH}$ radicals shown in Figure 3.9a are formed in the high temperature, exothermic reaction zones, and serve as a marker for the products and "post-flame zone" [121]. In all cases, OH fluorescence was consistently greater in magnitude along the leading edge of the flame front where $\mathrm{OH}$ is initially produced at superequilibrium levels. As $\mathrm{OH}$ concentrations decayed toward equilibrium away from the flame front, the fluorescence intensity decreased within the products of the $\mathrm{V}$-flame. When an equivalence ratio gradient was imposed, $\mathrm{OH}$ fluorescence decreased more rapidly away from the leading edge of the stratified flame front as richer mixtures near the stabilization point resulted in lower equilibrium $\mathrm{OH}$ concentrations downstream within the $\mathrm{V}$-flame. This decrease became more prominent as the gradient was increased, and the products within the V-flame were produced from progressively richer mixtures.

For the $\partial \phi / \partial y=-0.001 \mathrm{~mm}^{-1}$ reference premixed case, $\mathrm{OH}$ fluorescence was consistently present throughout the products with greater intensity in areas of positive curvature (convex toward the reactants) along the leading edge of the flame front. This may be an indicator of higher heat release due to a strong production of $\mathrm{OH}$ radicals, with which higher temperatures are generally associated [122]. As an equivalence ratio gradient was imposed along the flame front, variations in $\mathrm{OH}$ fluorescence intensity became more significant along the leading edge of the individual PLIF images, as apparent in the left column of Figure 3.9. While peak intensities in $\mathrm{OH}$ were comparable among all flame conditions, lower pixel intensities observed in areas of negative curvature (concave toward the reactants) dropped off more significantly in the richer flame regions as stratification increased. Cusps protruding into the products tended to be sharper and had lower $\mathrm{OH}$ fluorescence intensity. As the gradient was increased, $\mathrm{OH}$ values gradually decreased at the tip of the cusps until no $\mathrm{OH}$ was present, implying a decrease in the local reaction rate and quenching of the flame front. The formation of cusps is typical of rich iso-octane flames [123-125], as Darius Landau hydrodynamic instabilities are enhanced by thermo-diffusive effects $[126,127]$, and lead to the formation of cellular structures [123]. As seen previously in [44], these cusped formations were generally observed in the lower axial regions of the flame where richer mixtures with 
progressively lower Lewis numbers, Le, were present as the equivalence ratio gradient was increased. Conversely, the higher axial positions in the flame with progressively leaner mixtures and greater values of Le showed a smoothly wrinkled front with relatively larger positive curvatures toward the unburnt mixture and negative curvatures toward the products. While cusps were also occasionally observed at higher axial positions in the analysis ROI where the mean equivalence ratio ranged from $0.95 \leq \phi \leq 1.05$, they only formed in under $1.2 \%$ of cases for the steepest gradient case, and this was attributed to the presence of locally richer pockets that were entrained by turbulent mixing.

The $\mathrm{CH}_{2} \mathrm{O}$ fluorescence contours shown in the centre column of Figure 3.9 closely match the leading edge of $\mathrm{OH}$ contours, which is indicative of the accuracy of the spatial registration between $\mathrm{CH}_{2} \mathrm{O}$ and $\mathrm{OH}$ images. $\mathrm{CH}_{2} \mathrm{O}$ is produced in the low temperature oxidation process of the first stages of hydrocarbon combustion and is therefore a marker of low temperature reactions [122] and a good indicator of the cool-flame region [115]. For the premixed $\left(\partial \phi / \partial y=-0.001 \mathrm{~mm}^{-1}\right)$ flame setting, the $\mathrm{CH}_{2} \mathrm{O}$ fluorescence signals revealed a thin, low-temperature reaction zone with a sharply defined edge in both positive and negative curvature areas. As the mixture gradient was increased, pockets of slightly more diffuse $\mathrm{CH}_{2} \mathrm{O}$ were observed in cusped regions, which corresponded to areas where no $\mathrm{OH}$ signal was observed, suggesting the presence of cool-flame regions of lower temperature. Unlike in the $\mathrm{OH}$ images, small-scale intensity variations were observed along the $\mathrm{CH}_{2} \mathrm{O}$ contours. These local fluctuations were present in both positive and negative curvature zones and were attributed to shot noise which ranged between $11.4 \%$ and $20.7 \%$ at $95 \%$ confidence, compared to only $3.1 \%$ to $4.4 \%$ for the $\mathrm{OH}$ data.

The right column of Figure 3.9 shows corresponding instantaneous heat release rate images. Variations in HRR were observed in the bowed regions of negative curvature and cusps, where the $\mathrm{OH}$ signal was weakest and local HRR was generally lower. As stratification was increased, these low heat release rate areas tended to fade in intensity and eventually disappeared, resulting in the effective fracturing of the flame front, confirming the presence of local extinctions. Two such extinctions are highlighted in Figure 3.9 for flame condition $\partial \phi / \partial y=0.029 \mathrm{~mm}^{-1}$, and are consistent with the highly cusped flame structures observed in the locally rich $\mathrm{OH}$ images. 
Small-scale intensity variations were also observed along the individual HRR images (shown in Figure 3.9, and highlighted in Figure 3.10b), on the same scale as the $\sim 1 \mathrm{~mm}$ thickness of the instantaneous contours. These observations are potentially in line with DNS studies $[39,40,66-69]$ in which local variations in heat release rate along stratified flame fronts were observed, as well as experiments by Barlow et al. [36] and Sweeney et al. [31-34] who also observed significant gradients in equivalence ratio on the scale of the thermal flame thickness within the preheat zone of stratified flames. However, there is too much photon shot noise in the instantaneous heat release rate images to conclude that these small-scale local fluctuations in fluorescence intensity are necessarily evidence of variations in local reaction rate. 
$(\mathrm{OH}$ LIF)

Fluorescence intensity

$$
\text { [a.u.] }
$$

$\begin{array}{lllll}0.1 & 0.2 & 0.3 & 0.4 & 0.5\end{array}$
$\left(\mathrm{CH}_{2} \mathrm{O}\right.$ LIF $)$

Fluorescence intensity

[a.u. $\times 10^{2}$ ]
$\left(\mathrm{OH}\right.$ LIF) $\left(\mathrm{CH}_{2} \mathrm{O}\right.$ LIF $)$

Heat Release Rate

[a.u. $\times 10^{3}$ ]
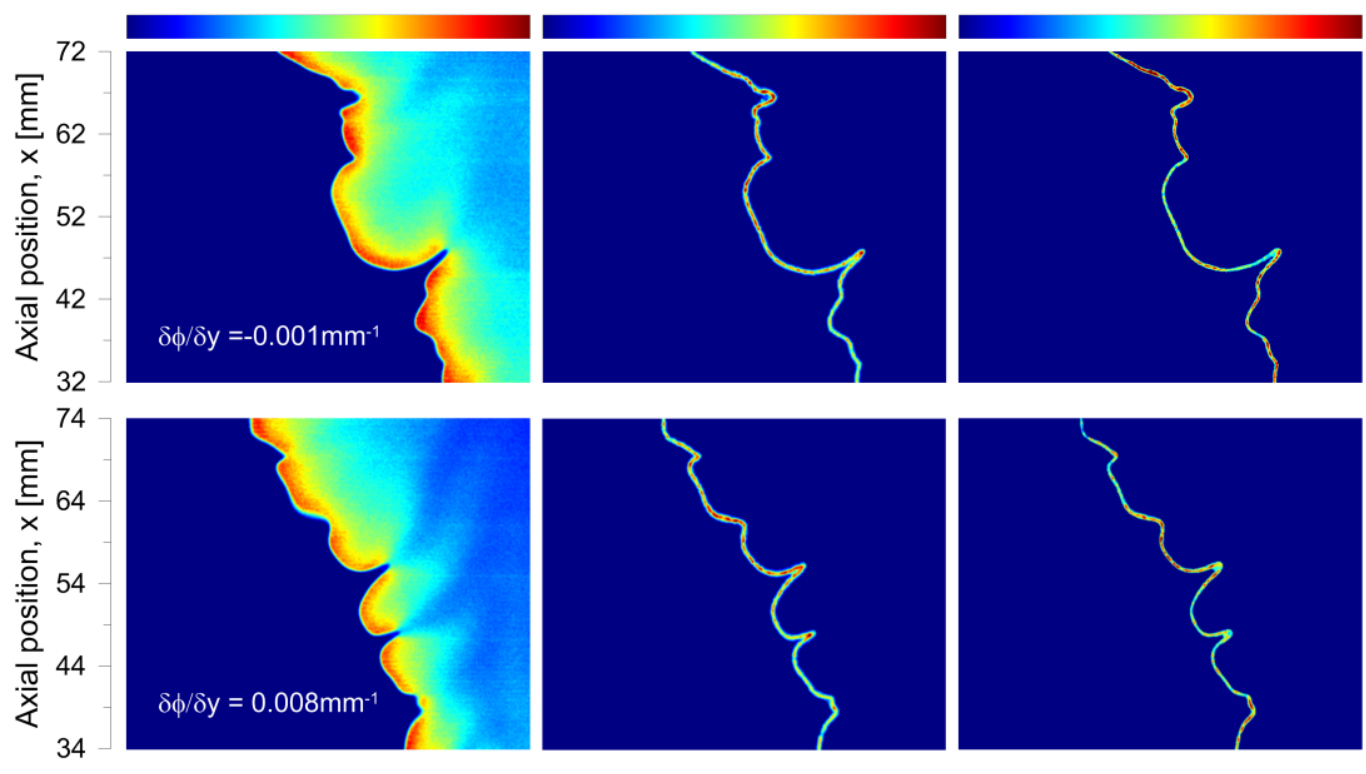

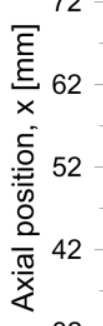
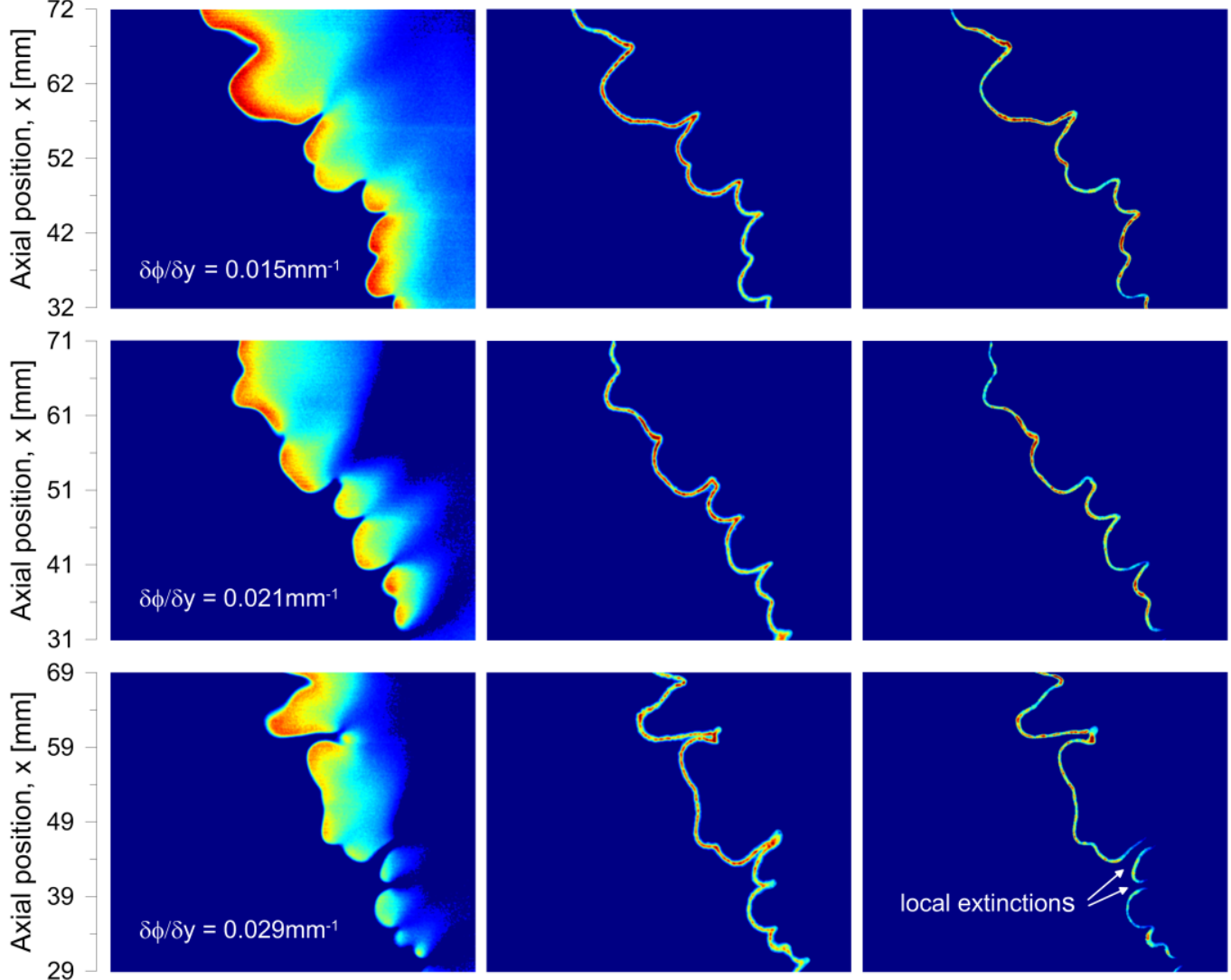

$\begin{array}{lllllllllllllll}-30 & -20 & -10 & 0 & 10 & -30 & -20 & -10 & 0 & 10 & -30 & -20 & -10 & 0 & 10\end{array}$

Transverse position, y $[\mathrm{mm}] \quad$ Transverse position, y [mm] Transverse position, y [mm]

Figure 3.9 Instantaneous PLIF images of $\mathrm{OH}$ (left), $\mathrm{CH}_{2} \mathrm{O}$ (centre), and relative heat release rate (right) for different equivalence ratio gradients. 


\subsubsection{Equivalence Ratio Gradient Effects on Heat Release Rate}

To further quantify the observed changes in the distribution of combustion intensity along the instantaneous flame fronts shown in Figure 3.9, the variation in local HRR was evaluated as a function of curvature. Curvature values were calculated at equally spaced intervals along the leading edge of each instantaneous HRR contour and matched with the peak heat release rate values extracted along a line normal to the leading edge. Figure 3.10a shows a sample HRR contour where the boxed region is magnified in Figure 3.10b. The white dotted lines in the latter image illustrate the local normals to the leading edge of the HRR contour, along which the peak HRR values were extracted.

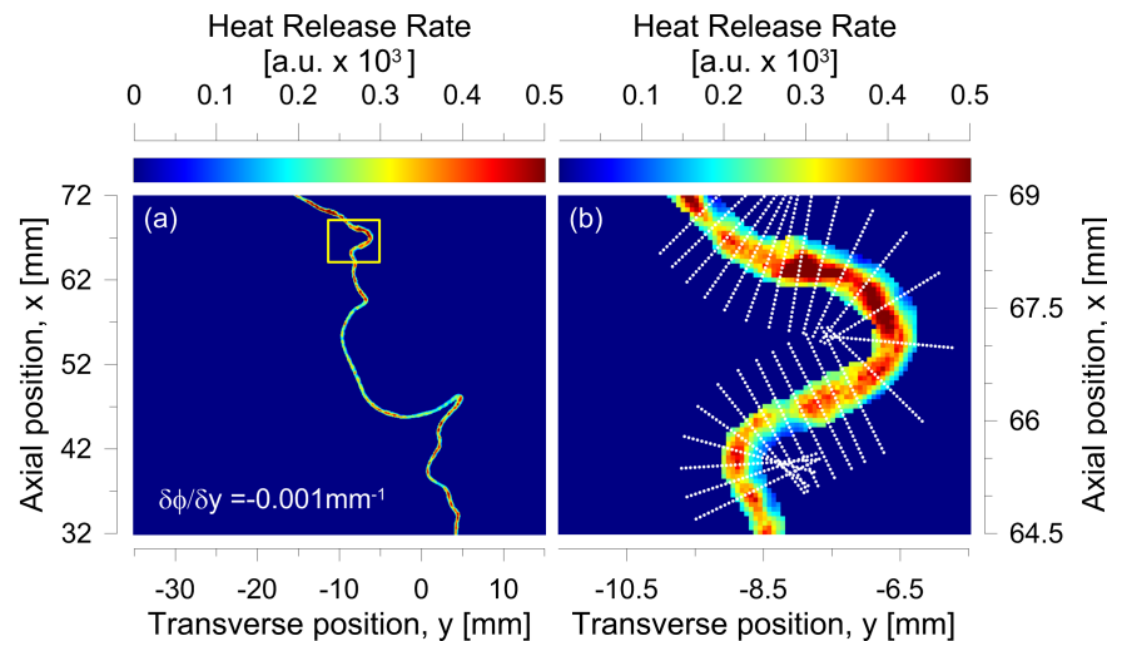

Figure 3.10 Sample instantaneous heat release rate contour for premixed flame condition $\delta \phi \delta y=$ $0.001 \mathrm{~mm}^{-1}$. Boxed region in (a) corresponds to the zoomed region shown in (b). White dotted lines in (b) indicate equally spaced locally normal profiles along the leading edge of the HRR contour. The peak HRR value from each profile was matched to the calculated curvature value at the leading edge.

Figure 3.11 plots these estimated HRR fluorescence intensity pixel values as a function of curvature for each stratified case alongside the reference premixed case. To ensure fair comparison between premixed and stratified flame conditions, the premixed case data were re-plotted using the different ROIs specific to each of the four stratified flame conditions (left column of Figure 3.11). The plots show a significant scatter in the data, as the local instantaneous HRR values include contributions from both photon shot noise of the (OH LIF) $\left(\mathrm{CH}_{2} \mathrm{O}\right.$ LIF) signals (see Figure 3.9), as well as flame/turbulence interactions. An asymmetrical distribution in HRR is observed for all flame conditions with a diffuse, left-protruding tail in areas of negative curvature. Comparing the premixed and stratified 
conditions, as the equivalence ratio gradient was increased, the negative tail became slightly more diffuse with a greater spread in HRR values. The rate of occurrence of low HRR pixels also gradually increased with stratification, until the onset of zero fluorescence intensity values indicating local extinctions along the flame front. These data points are highlighted in Figure 3.11 for flame conditions $\partial \phi / \partial y=0.021 \mathrm{~mm}^{-1}$ and $\partial \phi / \partial y=0.029 \mathrm{~mm}^{-1}$, and further support the existence of local extinctions apparent in the sample instantaneous HRR images shown in Figure 3.9. Within the region of interest, i.e between $0.95 \leq \phi \leq 1.05$, local extinctions corresponded to $<1.2 \%$ of data points, even for the most stratified flame condition, and as previously discussed, this was attributed to turbulent mixing that entrained locally rich pockets into the ROI. However, it is important to note that this interpretation changes significantly if no ROI is applied to the data and different ranges of equivalence ratios are instead considered when comparing measurements at different mixture gradients. In this latter case, the HRR vs. curvature distributions become significantly altered with stratification, with local extinctions occurring in up to $8.8 \%$ of data points for flame condition $\partial \phi / \partial y=0.029 \mathrm{~mm}^{-1}$. These plots are included in Section 3.6.3. 



Figure 3.11 Heat release rate vs. curvature for reference fully-premixed and stratified flame conditions with matching region of interest. 
Changes in local HRR pixel values were assessed by generating probability density functions of the local HRR excluding zero-valued pixels within the corresponding ROI for each flame condition. The plots in Figure 3.12 were obtained by normalizing the number of observations in each bin by the total number of non-zero HRR pixels for each flame condition, which varied significantly between flame settings because of the different width of each ROI.

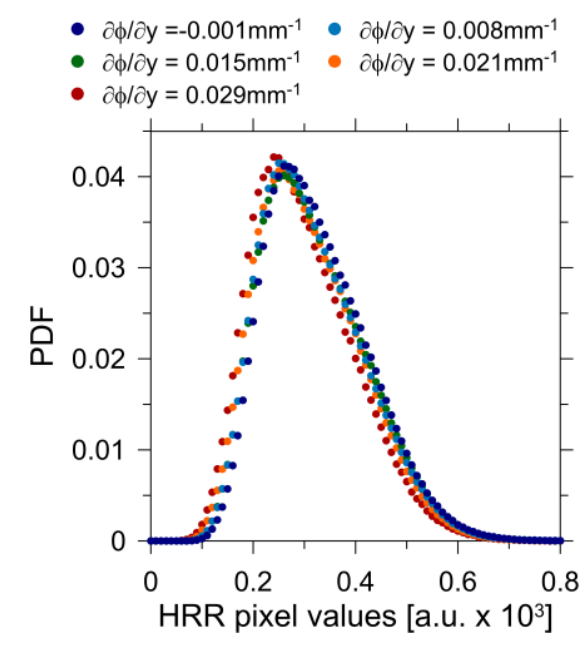

Figure 3.12 PDF of HRR fluorescence intensity for all gradient cases.

The profiles in Figure 3.12 show a small increase in the probability of low heat release rate pixels with steepening gradient as the profiles shift slightly to the left. HRR pixel values were consistently lower in all stratified cases than in the reference premixed case, consistent with the increase in low HRR pixel values also observed in Figure 3.11. The milder gradient cases $\left(\partial \phi / \partial y=-0.008 \mathrm{~mm}^{-1}\right.$ and $\partial \phi / \partial y=0.015 \mathrm{~mm}^{-1}$ ) showed a slight decrease in the mean HRR on the order of $3 \%$ compared to the reference premixed case, while the steeper gradient conditions $\left(\partial \phi / \partial y=0.021 \mathrm{~mm}^{-1}\right.$ and $\partial \phi / \partial \mathrm{y}=0.029 \mathrm{~mm}^{-}$ ${ }^{1}$ ) showed decreases of $5.9 \%$ and $8.6 \%$. Estimates of the variance of instantaneous HRR pixel values separate from system noise (which artificially broadens the distributions in Figure 3.12) were obtained following the same approach as for the equivalence ratio data. Given the weakly turbulent flow field, any variations in the local HRR due to flame stretch and strain (as a direct result of flame/turbulence interactions) are expected to vary negligibly among gradient flame conditions where the bulk flow conditions were unchanged. Within this context, relative to the premixed case, the coefficient of variation 
gradually increased with stratification from $7.3 \%$ to $9.8 \%$ from the weakest to steepest gradient cases, in line with the increase in reactant standard deviation from 0.029 to 0.081 and 0.031 to 0.080 along the $\phi=0.95$ and $\phi=1.05$ iso-contours of the ROI.

As detailed in Sections 3.3.1.1 and 3.3.2.1, decreases in mean (OH LIF) $\left(\mathrm{CH}_{2} \mathrm{O}\right.$ LIF) of up to $1.0 \%$, $1.6 \%, 2.7 \%$, and $4.3 \%$ could be expected for the weakest to steepest gradient cases as the standard deviation of $\phi$ within the ROI increased. However, the measured (OH LIF) $\left(\mathrm{CH}_{2} \mathrm{O}\right.$ LIF) variation was at least 2.0 to 3.4 times greater, implying a small but directly attributable decrease in HRR due to imposed mean $\phi$ gradient of up to $4.4 \%$ for the largest gradient case of $\partial \phi / \partial y=0.029 \mathrm{~mm}^{-1}$. While these differences are still small in the context of the inherent uncertainties of the diagnostic, it is important to note that this conclusion is apparent when comparing data for a consistent mean range of equivalence ratios (i.e. $0.95 \leq \phi \leq 1.05)$ that lie within the ROI, implying a specific gradient effect on mean HRR pixel values along the branches of the turbulent stratified V-flame. The observed decrease in local heat release rate of near stoichiometric flame regions suggests that heated products within the V-flame provide a form of "back-support" to the leading branches of the V-flame. For near stoichiometric flame regions, thermal back support is expected to decrease as the gradient steepens and the temperature of the adjacent heated products decreases for reactants of weaker mixture strength. The effect would be more significant for the steeper gradient cases, as the adiabatic flame temperature decreases away from its maximum at $\phi \approx 1.04$. This reasoning is generally analogous to that observed in laminar flame configurations $[21-26,70,72,80]$ in which a flux of heat and/or radicals within the products provide support to the flame, and can lead to an extension of flammability limits [21-26,70,72,80], and an increase in burning [70] and heat release rates $[72,80]$. In addition, variations in equivalence ratio along the flame front may also affect heat and species diffusion within the reaction zone. The presence of neighbouring flame regions of weaker mixture strength may provoke preferential diffusion away from the stronger stoichiometric regions, leading to a local decrease in HRR. A detailed schematic highlighting these mechanisms is provided in Section 3.6.4.

The effect of equivalence ratio gradient on the mean heat release rate distribution through the turbulent flame brush was separately evaluated by taking the ensemble average of the instantaneous fluorescence images (as in Figure 3.9) and plotting them as a function of the progress variable $\langle c\rangle$ within 
the corresponding ROI. The profiles in Figure 3.13a represent a measure of the mean heat release rate across the flame brush, in which the flame surface density, instantaneous thickness of the individual HRR contours, and local pixel values are all factors that contribute to the profiles. For all flame settings, the raw HRR fluorescence intensity profiles in Figure 3.13a are consistently skewed toward the products, comparable to those reported for flame surface density in stratified [31,45,47,48] and non-stratified [88] turbulent V-flames, as well as in stratified swirl burners [32,33]. The HRR for the fully-premixed flame peaked at an intensity of $\sim 0.28 \times 10^{-4}$ at $\langle\mathrm{c}\rangle \approx 0.6$, slightly downstream of the FSD peak. Figure $3.13 \mathrm{~b}$ reproduces flame surface density profiles reported in [47] using the refined analysis method detailed above, and shows negligible change in FSD with gradient, contrary to the slight increases previously observed. This suggests that given the current flow conditions, any change in net HRR is unlikely to be caused by a change in FSD.

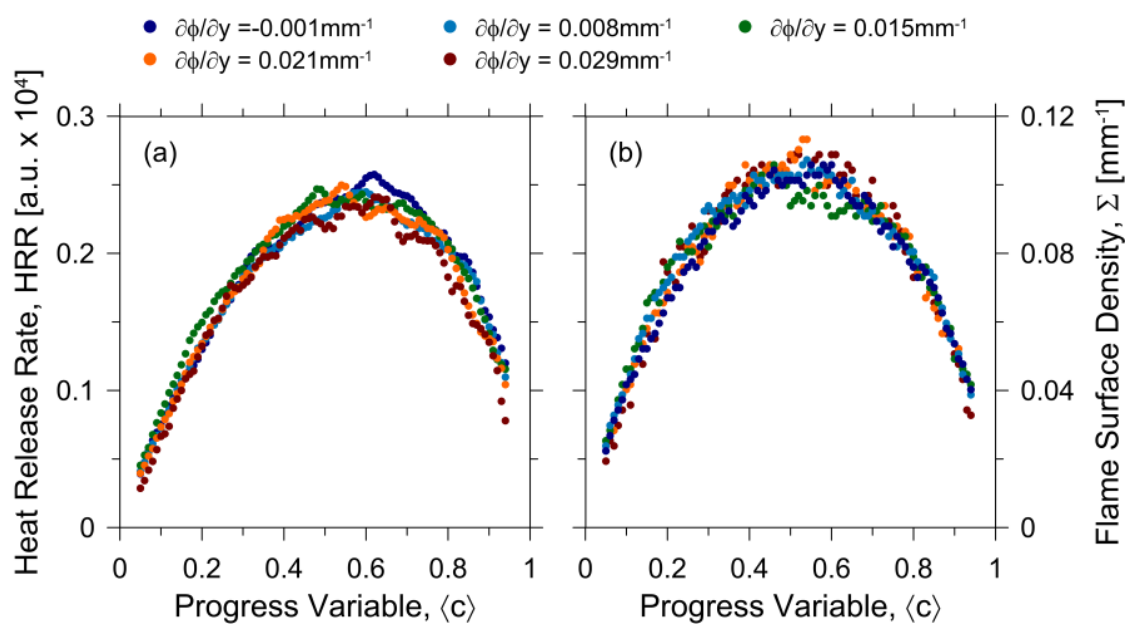

Figure 3.13 Windowed (a) heat release rate fluorescence intensities and (b) flame surface density as a function of progress variable $\langle c\rangle$ for reference premixed and stratified flames.

Although the relative heat release rates are slightly greater in the reference premixed case, variations with gradient remained small, consistent with the decrease in mean HRR pixel value reported in Figure 3.12. Reductions in HRR were only slightly more apparent on the product side of the flame brush $(\langle c\rangle \geq 0.5)$, where a greater decrease was observed, in line with the weaker HRR in the negative curvature regions of the flame shown in Figure 3.11. The observed subtle changes in distribution of HRR across the 
flame brush suggest that while decreases in the mean HRR do occur, the net effect of $\phi$ gradients on locally stoichiometric flame regions remains small for the current experimental conditions.

\subsubsection{Global Stratification Effects}

The effect of stratification on the overall behaviour of the flame is evaluated by comparing the reference premixed case to the stratified cases while analysing the entire flame image (i.e. without fixed $\phi$ range ROI) between axial positions $34.2 \leq \mathrm{x} \leq 69.5 \mathrm{~mm}$ above the exit of the burner. This resulted in a progressively broader range of $\phi$ as the gradient was increased, such that for the steeper gradient cases, both front and back supported flame regions are expected to occur based on the location of the maximum HRR and $\mathrm{S}_{\mathrm{L}}$ at $\phi \approx 1.07$ shown in Figure 3.14. Data were analysed for variations in the ensemble average of 2D flame lengths extracted from instantaneous flame contours and the ensemble average of the mean HRR excluding zero valued pixels along the flame contours. The effect of local extinctions on the ensemble-average of HRR per flame length was also evaluated, in addition to the ensemble-averaged total HRR, which represents the product of the 2D flame length and the local HRR.

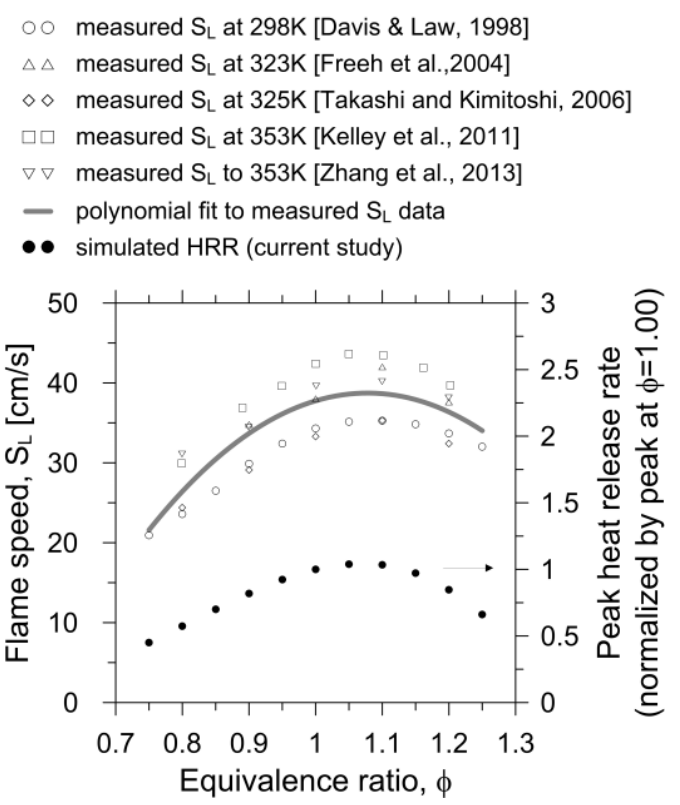

Figure 3.14 Experimental laminar flame speed $S_{L}$ [95-99], polynomial fit to experimental $S_{L}$ data, and simulated peak heat release rate for premixed flames ranging from $\phi=0.75-1.25$. 
The ensemble-averaged instantaneous flame length (i.e. the length along the instantaneous 2-D turbulent flame contour) was normalized by the ratio of the length of the $\langle c\rangle=0.5$ contour line between the stratified and premixed flame conditions. This accounts for differences in measured flame length that are attributed to the mean flame brush incident angle, and ensures variations in flame length are physical in nature rather than an artefact of the interrogation region within the V-flame.

Table 3.2 Total flame length and heat release rate per instantaneous image.

\begin{tabular}{|c|c|c|c|c|}
\hline $\begin{array}{c}\text { Equivalence ratio } \\
\text { gradient, } \\
\delta \phi / \delta \mathrm{y} \\
{\left[\phi \mathrm{mm}^{-1}\right]}\end{array}$ & $\begin{array}{c}\text { Ensemble-averaged } \\
\text { instantaneous flame } \\
\text { length }[\mathrm{mm}]\end{array}$ & $\begin{array}{l}\text { Ensemble average } \\
\text { of the mean HRR* } \\
\text { excluding zero- } \\
\text { valued pixels } \\
\text { [a.u. } \times 10^{-4} \text { ] }\end{array}$ & $\begin{array}{c}\text { Ensemble average } \\
\text { of HRR } * \text { flame } \\
\text { length } \\
\text { [a.u. x } 10^{-2} / \mathrm{mm} \text { ] }\end{array}$ & $\begin{array}{c}\text { Ensemble average } \\
\text { of the total HRR* in } \\
\text { each instantaneous } \\
\text { image } \\
\text { [a.u.] }\end{array}$ \\
\hline-0.001 & 61.7 & 3.12 & 2.55 & 1.57 \\
\hline 0.008 & 64.2 & $3.00-3.00$ & $2.44-2.44$ & $1.57-1.57$ \\
\hline 0.015 & 65.3 & $2.92-2.92$ & $2.45-2.45$ & $1.60-1.60$ \\
\hline 0.021 & 69.5 & $2.72-2.74$ & $2.12-2.14$ & $1.47-1.48$ \\
\hline 0.029 & 72.3 & $2.61-2.63$ & $1.98-1.99$ & $1.43-1.44$ \\
\hline
\end{tabular}

Table 3.2 shows a monotonic increase in the mean flame length of individual flame realisations as stratification increases, with the increase becoming more significant for the steeper gradients, up to $17.2 \%$ relative to the reference homogenous case, consistent with the excess wrinkling observed in the individual images in Figure 3.9. Conversely, the mean HRR excluding zero-valued pixels decreased with stratification, leading to a measured reduction of $15.6-16.2 \%$ for the steepest gradient case, attributable to the presence of lower mixture strengths between $0.74 \leq \phi \leq 1.22$ along the flame front. This contrasts with a $4.3-4.4 \%$ decrease based on the effect of gradient alone for a near-stoichiometric flame region, as determined by using the ROI approach discussed in Section 3.4.2. The onset of local extinctions further reduced the mean HRR along the reacting front, such that the ensemble-averaged HRR/flame length decreased by up to $21.9-22.5 \%$ for the steepest gradient relative to the reference premixed case. .

Despite significant variations in mean flame length and HRR/flame length, the net effect of stratification on the total heat release rate remains small, highlighting the influence of both mechanisms on 
the total heat release rate in the context of a V-flame, which is in line with the numerical results of [39,40,66-69]. This suggests that for weaker gradients, given the current globally stoichiometric turbulent conditions, stratification induced flame surface generation is offset by the decrease in HRR per flame length. Conversely, for steeper gradients, the occurrence of local extinctions, in addition to the presence of weaker mixture strengths, results in a small $8.4-9.1 \%$ decrease in total heat release rate.

\subsection{Conclusion}

The effects of stratification on the heat release rate in a globally stoichiometric turbulent iso-octane/air Vflame were experimentally studied by comparing a reference premixed case to four mean equivalence ratio gradient flame conditions. The $(\mathrm{OH} \mathrm{LIF})\left(\mathrm{CH}_{2} \mathrm{O}\right.$ LIF) approach to HRR measurements was validated in stratified iso-octane/air flames following detailed numerical simulations of 1D laminar premixed flames over a broad range of equivalence ratios. Potential temperature effects on the $\mathrm{OH}$ and $\mathrm{CH}_{2} \mathrm{O}$ fluorescence signals were quantified by considering the Boltzmann population fractions and collisional quenching rates of both species for the excitation transitions used in the experiments.

Instantaneous heat release rate images, obtained from the product of spatially registered $\mathrm{OH}$ and $\mathrm{CH}_{2} \mathrm{O}$ PLIF images, revealed the formation of cusped structures with qualitatively low HRR values in areas of negative curvature along individual flame contours. These structures were generally observed in locally rich flame regions, and were attributed to Darius Landau hydrodynamic instabilities that were enhanced by thermo-diffusive effects. As stratification was increased, these cusps led to occasional local extinctions along the flame front, where slightly more diffuse $\mathrm{CH}_{2} \mathrm{O}$ was observed in areas with no $\mathrm{OH}$ signal.

The effects of large-scale gradients in equivalence ratio were evaluated using a windowing technique that quantifies specific mean gradient effects on flame regions with a constant mean range of mixture strengths. Decreases in estimated HRR of up to $4.4 \%$ relative to the reference premixed case were directly attributable to the imposed mean $\phi$ gradient (up to $\partial \phi / \partial y=0.029 \mathrm{~mm}^{-1}$ ) within a nearstoichiometric region of interest (ROI). These small but detectable changes in mean HRR were 2.0-3.4 times greater than potential effects of variations in temperature and local mixture strength within the ROI. The decrease in HRR due to $\phi$ gradient was attributed to the decrease in thermal back support from the heated products within the V-flame, as the temperature of adjacent products would be expected to decrease 
for reactants of weaker mixture strength. When plotted as a function of the reaction progress variable $\langle\mathrm{c}\rangle$, a slight decrease in the mean estimated HRR was observed on the product side of the flame brush where $\langle c\rangle \geq 0.5$. These results suggest that under the current flow conditions and imposed gradient levels, the net effects specific to mean equivalence ratio gradients on locally near-stoichiometric flame regions are apparent, but small relative to the limits of the current (OH LIF) $\left(\mathrm{CH}_{2} \mathrm{O}\right.$ LIF) diagnostic.

Global stratification effects were separately evaluated by comparing globally stoichiometric mixtures with different ranges in equivalence ratio. Effects were much stronger in this scenario, revealing competing effects of enhanced surface generation (17.2\% increase) and reduced HRR/flame length (21.9$22.5 \%$ ), resulting in a small reduction in total heat release rate of up to $8.4-9.1 \%$. This experimentally supports numerical predictions $[39,40,66-69]$ in which both mechanisms were found to contribute to the overall behaviour of the flame. The results presented here stress the importance of clearly defining the question and measurement approach when attempting to discern specific effects of stratification on combustion. Gradient effects on local flame properties may differ significantly from general stratification effects in which different ranges of mixture strengths are present, and separating these subjects is critical to the proper interpretation of data, where both may be relevant in different applications.

\subsection{Supplemental Material}

\subsubsection{Equivalence Ratio Measurements}

To correlate the fluorescence intensity to equivalence ratio measurements of the reactant mixture, homogeneous reference images were acquired at known mixture strengths. One thousand individual images were acquired at five premixed reactant settings, $\phi=0.4, \phi=0.7, \phi=1.0, \phi=1.3$, and $\phi=1.6$. Images were processed following the exact methodology outlined in Section 3.3.1, and corrected for background intensity, mean laser profile, and pulse-to-pulse variations in laser energy before a $5 \times 5$ pixel median filter was applied. The average fluorescence intensity of a $2 \times 2 \mathrm{~cm}^{2}$ interrogation region centered transversely at $\mathrm{y}=0.0 \mathrm{~cm}$ and axially at $\mathrm{x}=2.0 \mathrm{~cm}$ was determined for each individual image. The 1000 mean values for each of the three reference premixed cases were then ensemble averaged and plotted, as shown in Figure 3.15. A weighted linear regression function was then fitted to relate fluorescence intensity (measured in pixel counts) to equivalence ratio. The $95 \%$ confidence intervals were also calculated. 


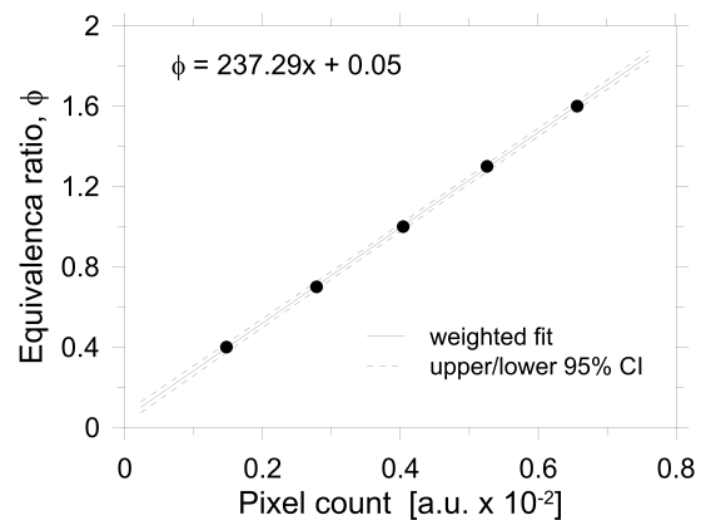

Figure 3.15 Weighted linear correlation function of fluorescence intensity (measured in pixel counts) to equivalence ratio and upper/lower $95 \%$ confidence intervals.

\subsubsection{Correlation between (OH LIF)( $\mathrm{CH}_{2} \mathrm{O}$ LIF) and k(T)[OH][CH$\left.{ }_{2} \mathrm{O}\right]$ Signals}

To complement the correlation of $(\mathrm{OH} \mathrm{LIF})\left(\mathrm{CH}_{2} \mathrm{O}\right.$ LIF) with HRR shown in Figure 7 of the manuscript, the same data have been re-plotted as a function of the forward reaction rate $\mathrm{k}(\mathrm{T})[\mathrm{OH}]\left[\mathrm{CH}_{2} \mathrm{O}\right]$. Figure 3.16a and Figure 3.16b plot the estimated (OH LIF) $\left(\mathrm{CH}_{2} \mathrm{O}\right.$ LIF) signals as a function of $\mathrm{k}(\mathrm{T})[\mathrm{OH}]\left[\mathrm{CH}_{2} \mathrm{O}\right]$ as they vary of through the flame for $\mathrm{CH}_{2} \mathrm{O}$ quenching models $\mathrm{T}^{-0.5}$ and $\mathrm{T}^{-1.0}$ respectively. The loops are slightly tighter than those of $(\mathrm{OH} \mathrm{LIF})\left(\mathrm{CH}_{2} \mathrm{O}\right.$ LIF) vs. HRR shown in Figure 3.7, suggesting that the forward reaction rate of $\mathrm{OH}+\mathrm{CH}_{2} \mathrm{O} \rightarrow \mathrm{HCO}+\mathrm{H}_{2} \mathrm{O}$ does not have a perfect correspondence with $\mathrm{HRR}$. The resulting temperature induced bias errors on the fluorescence measurement were calculated by taking the ratio of the integration and peak of the $(\mathrm{OH} \mathrm{LIF})\left(\mathrm{CH}_{2} \mathrm{O}\right.$ LIF) and $\mathrm{k}(\mathrm{T})[\mathrm{OH}]\left[\mathrm{CH}_{2} \mathrm{O}\right]$ profiles for both $\mathrm{CH}_{2} \mathrm{O}$ quenching models. Calculated values were then expressed as a function of the ratio at $\phi=1$ in Figure 3.16c. 


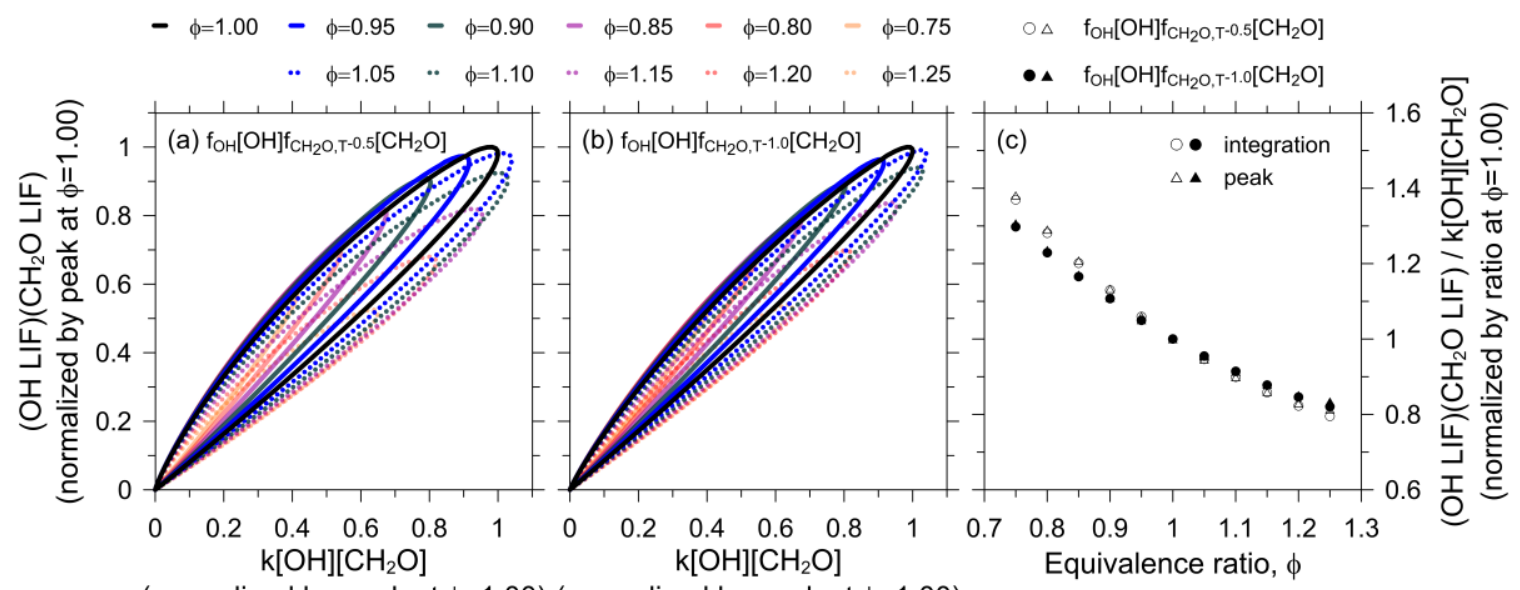

(normalized by peak at $\phi=1.00$ ) (normalized by peak at $\phi=1.00$ )

Figure 3.16 Correlation of $(\mathrm{OH} \mathrm{LIF})\left(\mathrm{CH}_{2} \mathrm{O} \mathrm{LIF}\right)$ with the forward reaction rate $\mathrm{k}[\mathrm{OH}]\left[\mathrm{CH}_{2} \mathrm{O}\right]$ for $\mathrm{CH}_{2} \mathrm{O}$ quenching models $\mathrm{T}^{-0.5}$ in (a) and $\mathrm{T}^{-1.0}$ in (b) for simulated premixed flames ranging from $\phi=0.75-1.25$. Variation of the ratio of integrated and peak $(\mathrm{OH} \mathrm{LIF})\left(\mathrm{CH}_{2} \mathrm{O} \mathrm{LIF}\right) / \mathrm{k}[\mathrm{OH}]\left[\mathrm{CH}_{2} \mathrm{O}\right]$ over a range of equivalence ratios is presented in (c).

\subsubsection{Non-Windowed HRR vs Curvature}

Figure 3.17 shows the variation in peak HRR along instantaneous flame contours plotted against spatially corresponding curvature. All data are plotted without using the windowing technique described in Section 3.3.1 so that unlike the plots shown in Figure 3.11, these supplemental plots each include different ranges of equivalence ratios. Variations are significantly more prominent than those observed for windowed data in Figure 3.11, as the general shape of the "clouds" changes with stratification. 

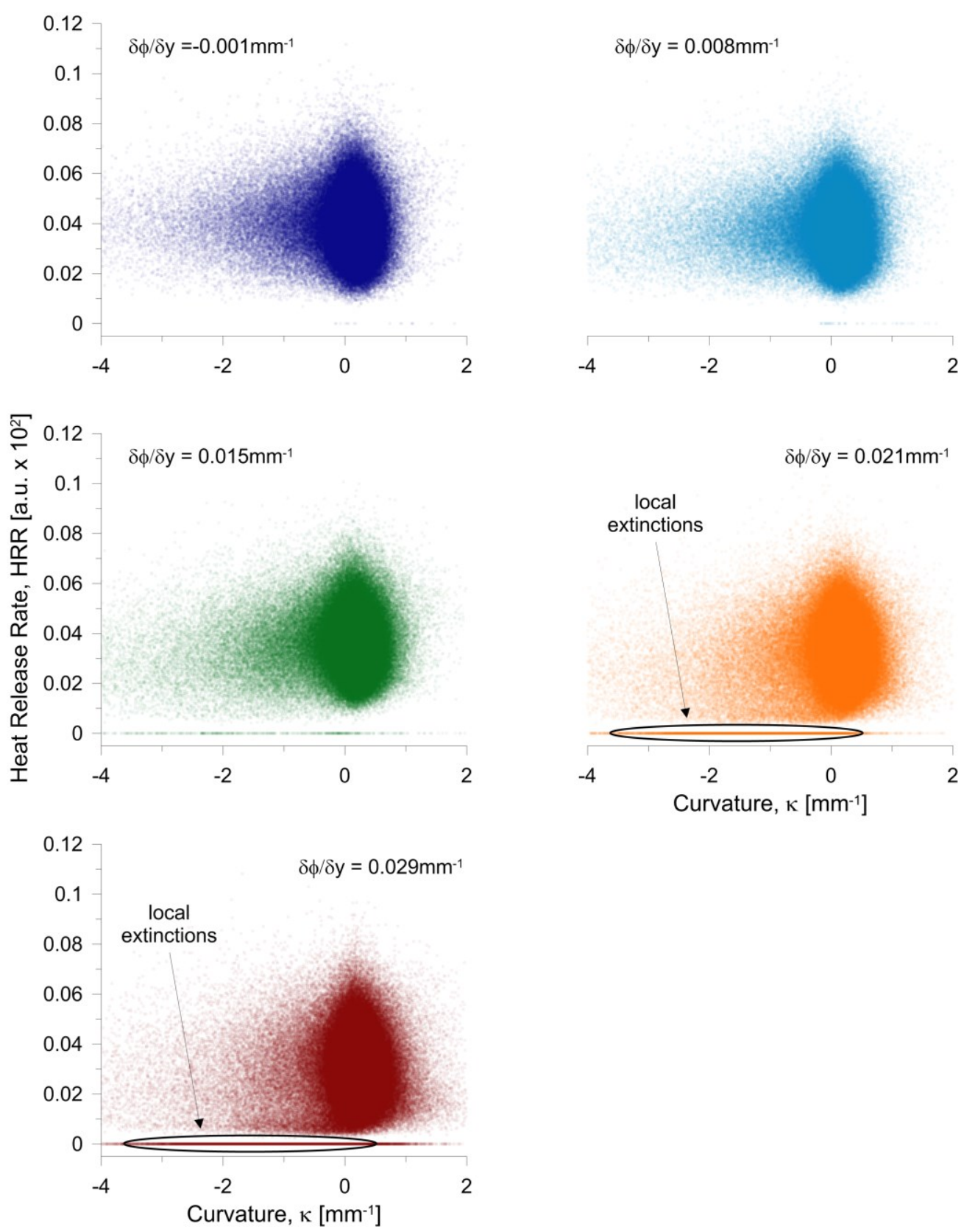

Figure 3.17 Heat release rate vs. curvature for reference premixed and stratified flame conditions plotted without using the windowing technique. 


\subsubsection{Physical Representation of the Effects of Mean $\phi$ Gradients on the Local HRR of Near-Stoichiometric Flame Regions Within the ROI}

In Section 3.4.2, a decrease in the HRR of near stoichiometric flame regions was observed as the mean $\phi$ gradient increased. The green arrows in Figure 3.18 highlight the anticipated variation in local equivalence ratio along the stratified flame front from relatively weak, Figure $3.18 \mathrm{a}$, to relatively steep, Figure $3.18 \mathrm{~b}$, mean $\phi$ gradients. The blue arrows represent the resulting progressive decrease in adiabatic flame temperature between these gradient conditions. This decrease in temperature leads to an inherent decrease in thermal back support (i.e. heat flux, red arrows) to the stronger, near-stoichiometric flames within the ROI, as well as an increase in preferential diffusion (i.e. heat and species flux, orange arrows) along the flame front away from the ROI. These mechanisms become more evident as the gradient increases, as highlighted by the different sized arrows in Figure 3.18a and Figure 3.18b.

(a) HRR $\downarrow$
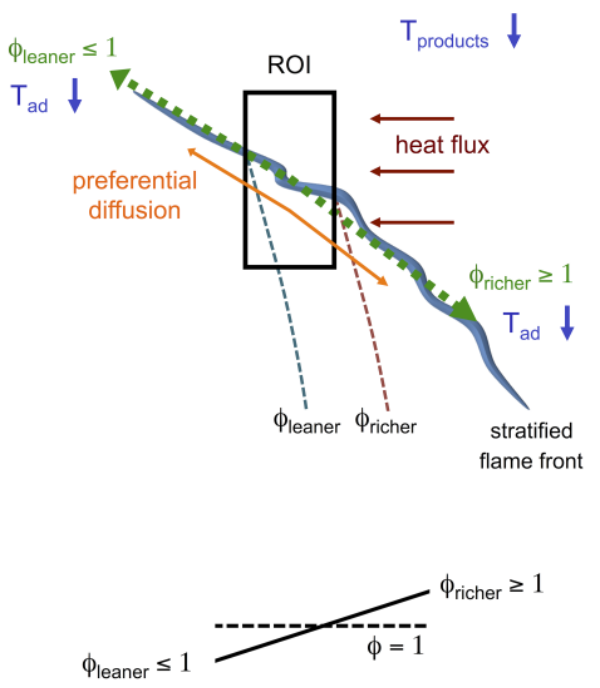

(b) HRR

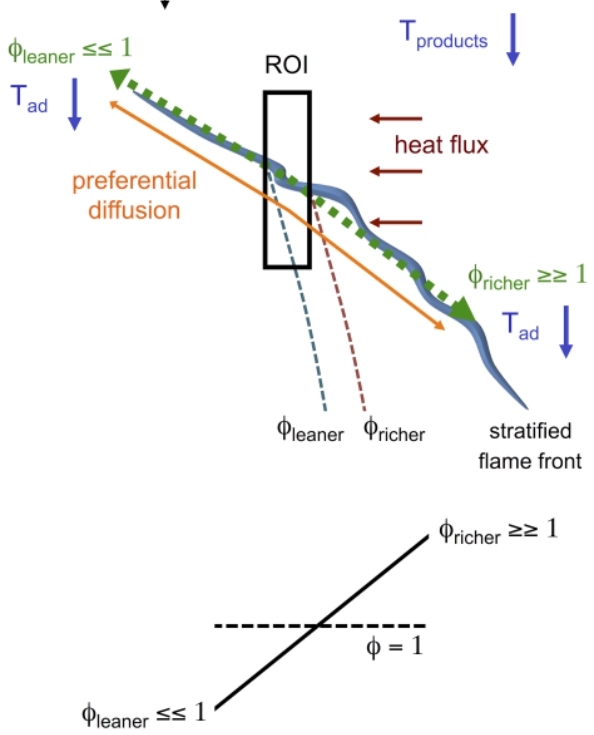

Figure 3.18 Schematic illustrating the anticipated effect of mean $\phi$ gradients on thermal back support (heat flux) to and preferential diffusion (heat and species flux) away from near stoichiometric flame regions $0.95 \leq \phi \leq 1.05$ within a ROI along a stratified flame front as the gradient steepens from (a) to (b). 


\section{Chapter 4}

\section{A Comparative Analysis on the Effects of Stratification on Locally Lean, Near-Stoichiometric, and Rich Iso-Octane/Air Turbulent V-Flames}

This chapter has been written as a paper intended for future submission to Combustion and Flame.

Vena, P.C., Deschamps B., Johnson, M.R. "A comparative analysis on the effects of stratification on locally lean, near-stoichiometric, and rich iso-octane/air turbulent V-flames", draft manuscript to be submitted to Combustion and Flame.

This paper was co-authored by the thesis author, Patrizio Vena, Dr. Béatrice Deschamps, and Prof. Matthew Johnson. Mr. Vena set up, tested, and characterized the measurement equipment; performed the measurements, uncertainty analysis, and initial data analysis; and wrote a first draft of the manuscript. Dr. Deschamps helped in the design of the experiments. The theory development, final data analysis, and editing and revision of the manuscript were conducted jointly by Mr. Vena and Prof. Johnson.

\subsection{Abstract}

The effects of partial premixing on locally rich, near-stoichiometric, and lean flame regions were evaluated in stratified, iso-octane/air turbulent V-flames by varying the mean equivalence ratio gradient along the exit plane of a rectangular exit burner. Instantaneous heat release rate (HRR) images were obtained from the product of spatially registered, near-simultaneously acquired $\mathrm{OH}$ and $\mathrm{CH}_{2} \mathrm{O}$ planar laser induced fluorescence (PLIF) images. HRR data were analyzed within a region of interest (ROI) that was determined from separate 3-pentanone tracer PLIF measurements. The ROI was unique to each gradient flame setting, and was configured to ensure the mean range of equivalence ratios being analyzed was constant among gradient conditions. This allowed distinction of the specific effects of mean equivalence ratio gradient from those of varied mixture strength.

Individual flame realizations were studied for differences in the local peak HRR and instantaneous flame thickness $\delta_{\mathrm{t}}$ as they varied with curvature among gradient conditions. Subtle changes in the 
normalized distribution of local peak HRR vs. curvature were observed for flames propagating in positive $(\delta \phi / \delta y>0)$ and negative $(\delta \phi / \delta y<0)$ mean $\phi$ gradients. Differences were strongest in locally rich and locally lean flames, and provided evidence of the potential interaction between neighbouring flame regions. The results suggest that $\phi$ gradients can affect the variation of peak HRR with curvature, and may influence the local thermodiffusive stability of the stratified, turbulent flame front.

Ensemble averages of individual peak HRR and $\delta_{\mathrm{t}}$ values within each ROI were separately evaluated, and differences among gradient conditions were greater than those observed for the normalized distributions with curvature. Opposing behaviour was observed between the peak HRR and $\delta_{\mathrm{t}}$ for all flame settings considered, as an increase of one led to a decrease of the other. Gradient effects were observed when comparing back- and front-supported locally rich flames, which experienced changes in in peak HRR up to $+10.2 \%$ and $-5.3 \%$ for gradient settings $\delta \phi / \delta y=-0.014 \mathrm{~mm}^{-1}$ and $\delta \phi / \delta y=0.012 \mathrm{~mm}^{-1}$ respectively, coupled with a thinning and thickening of $\delta_{\mathrm{t}}$ of up to $-7.2 \%$ and $+2.4 \%$. Similar but weaker trends were observed for near-stoichiometric flame regions, with a decrease in peak HRR of up to $-3.5 \%$ and a thickening up to $+2.8 \%$ for the steepest gradient $\delta \phi / \delta y=0.029 \mathrm{~mm}^{-1}$. Locally lean flames showed small increases in peak HRR of up to $+3.8 \%$, and decreases in $\delta_{\mathrm{t}}$ of up to $-2.1 \%$ for back-supported gradient case $\delta \phi / \delta \mathrm{y}=0.024 \mathrm{~mm}^{-1}$. Variations were not as significant as those observed for back-supported rich flame regions of equivalent gradients, suggesting that different mechanisms of back support (species or heat) may occur based on local stoichiometry.

Keywords: Stratified combustion, Turbulent combustion, V-flame, Equivalence ratio gradient, Planar Laser Induced Fluorescence, Heat release rate 


\subsection{Introduction}

Recent experiments $[31-34,36,47,128]$ have highlighted the importance of clearly defining the question and approach with which the effects of partial premixing are quantified, where it is important to differentiate the general effects of stratification on the overall behaviour of a flame system from the specific effects of equivalence ratio gradient on local flame properties. Consideration of the latter involves quantifying the interaction between neighbouring flame regions of differing mixture strength by observing variations in local flame properties. Flame configurations in which the local variation in mixture strength (either normal or tangential to the reaction zone) is precisely controlled have shed light on the underlying physical mechanisms that govern stratified flames. Several authors have approached this problem by devising controlled experiments $[21-25]$ and simulations $[26,70,72,80]$ in which an equivalence ratio gradient can be imposed normal to flames propagating in a channel [22-26], to spherically-expanding laminar flames [21], or to stagnation flames $[70,72,80]$. Results of these works have shown that diffusive fluxes of heat/mass from downstream products may back support flames of weaker mixture strength. Additionally, numerical simulations have demonstrated that tangential variations in mixture composition along the flame front $[39,40,66-69]$ can affect local combustion intensity, inducing differences in local flame speed $\mathrm{S}_{\mathrm{L}}^{\mathrm{o}}$. These flame speed variations may further wrinkle the flame front for $\mathrm{u}^{\prime} / \mathrm{S}_{\mathrm{L}}^{o}<1[39,40,66]$, although this effect is less significant as turbulence increases $\mathrm{u}^{\prime} / \mathrm{S}_{\mathrm{L}}^{\mathrm{o}}>1[39,66-69]$.

Isolating general effects of stratification from specific effects of local equivalence ratio gradient in fully turbulent experimental configurations is inherently more complex. However, recent advances in multi-component optical diagnostics are enabling investigation of the physical mechanisms that may alter stratified flame behaviour, as well as acquisition of validation data for numerical models. Working on a coannular weak-swirl burner, Bonaldo et al. [35] separately acquired 3-D velocity data using Stereoscopic Particle Image Velocimetry (SPIV), instantaneous temperature data from Rayleigh scattering, and mean local equivalence ratio data using acetone PLIF. They observed a broadening of flame curvature PDFs coupled with a shift towards more positive curvatures with stratification, in addition to a decrease in instantaneous thermal flame thickness. Seffrin et al. [51] introduced a novel axisymmetric stratified burner and fully characterized its turbulent flow field with Laser Doppler Velocimetry (LDV) and high-speed PIV. In subsequent work, Böhm et al. [46] used Rayleigh scattering and simultaneous $\mathrm{OH}$ and acetone PLIF to 
investigate variations in flame front thickness, curvature, and length with stratification. Kuenne et al. [52] then performed one dimensional Raman/Rayleigh scattering measurements of local temperature and species concentrations to validate Large Eddy Simulations (LES).

Barlow et al. [36] developed a system in which Raman/Rayleigh scattering and CO LIF were used for instantaneous line measurements of temperature and species concentration through the flame. Crossplanar OH PLIF was simultaneously acquired for 3D topology information. They successfully applied their approach to locally lean methane/air turbulent V-flames [31,36] and swirl-burners [32-34], and provided data conditioned on local instantaneous equivalence ratio [31-34] and equivalence ratio gradient [34], reporting instantaneous and mean values of local species concentration, temperature, and three-dimensional flame topology. Among other findings, their results suggest that stratification may lead to differences in local flame structure, which include observed gradients in equivalence ratio within the thermal ramp of the reaction zone.

In previous work by the present authors $[47,128]$, a different but complementary approach was developed in which 3-pentanone tracer PLIF is used to identify mean conditioned iso-contours of equivalence ratio $(\phi)$ up to the mean position of the flame-front at $\langle\mathrm{c}\rangle=0.5$. Subsequent simultaneous $\mathrm{OH}$ and $\mathrm{CH}_{2} \mathrm{O}$ PLIF measurements were used to probe flame topology and heat release rates within similar $\phi$ iso-contours for flame cases of differing mean $\phi$ gradient. By choosing an analysis region of interest (ROI) such that a constant mean range of $\phi$ is evaluated as the $\phi$ gradient is varied, it is possible to isolate specific gradient effects from those of varying mixture strength. This ROI approach has been applied to turbulent iso-octane/air V-flames in locally near-stoichiometric [47,128] flame regions. Results showed small but discernable variations in mean heat release rates among gradient conditions, and implied that "backsupport" from the heated products within the V-flame scaled with gradient [128], in line with the backsupport noted in flames subjected to gradients normal to the flame front in experiments [21-25] and simulations $[26,70,72,80]$.

The current study builds directly on this previous work and compares the behaviour of front- and back-supported rich, near-stoichiometric, and back-supported lean flame regions. Local peak HRR intensities and instantaneous flame thicknesses $\delta_{\mathrm{t}}$ along individual flame contours were evaluated and 
plotted in terms of their relative variation with local curvature in the presence of varying $\phi$ gradients. Measurement uncertainties of the local curvature and instantaneous HRR profile are considered in a Monte Carlo analysis. In addition, relative variations in the ensemble average of local peak HRR and instantaneous flame thicknesses $\delta_{\mathrm{t}}$ were compared among gradient and reference-premixed flame conditions. Using the previously described ROI technique, specific effects of mean equivalence ratio gradients are quantified, while ensuring unbiased comparison between reference-premixed and increasingly stratified flame conditions.

\subsection{Methodology}

Turbulent stratified iso-octane/air V-flames were stabilized above a stratified flame burner previously described in $[47,128]$. The burner generates a smooth, transverse gradient in equivalence ratio along the $63 \mathrm{~mm} \times 15 \mathrm{~mm}$ rectangular exit that is controlled by independently manipulating two premixed reactant streams, $\phi_{1}$ and $\phi_{2}$, as they entered the base of the burner. The exit nozzle was surrounded by a $4 \mathrm{~mm}$ air co-flow. Reactant air was pre-heated to $80^{\circ} \mathrm{C}$ to aid pre-evaporation of the iso-octane fuel, resulting in a temperature of $55^{\circ} \mathrm{C}$ at the exit plane of the burner.

Instantaneous flame realizations were obtained from the near-simultaneous acquisition of $\mathrm{OH}$ and $\mathrm{CH}_{2} \mathrm{O}$ planar laser induced fluorescence (PLIF) images. The optical PLIF setup shown in Figure 4.1 consisted of a dual head Quanta Ray PIV400 YAG laser coupled with a Sirah Precision Scan Rhodamine B

Dye laser. Fluorescence images were acquired using a pair of Princeton Instrument PIMAX 1340 x 1300 pixel intensified CCDs with ST-133 controllers. Images had a projected spatial resolution of $67 \mu \mathrm{m} /$ pixel. The line spread function of the optical system was 3.91 pixels (equivalent to $263 \mu \mathrm{m}$, as determined from the scanning knife edge method [129] detailed in Appendix A). Detailed descriptions of the burner and optical setup are provided in $[47,128]$. 


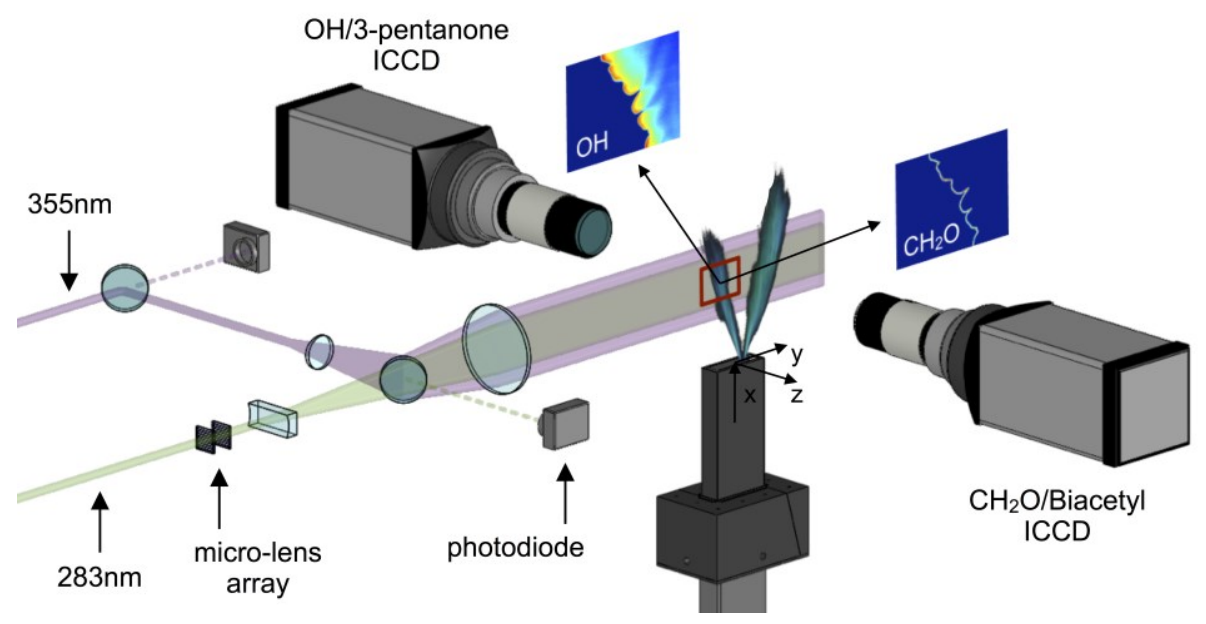

Figure 4.1 PLIF setup for simultaneous $\mathrm{OH}$ and $\mathrm{CH}_{2} \mathrm{O}$ acquisition.

Turbulent V-flames were anchored with a $1.5 \mathrm{~mm}$ diameter rod. Potential differences in local peak HRR and flame thickness as a function of imposed $\phi$ gradients were investigated for three main flame conditions corresponding to three different mean mixture strengths $(\bar{\phi}=1.2, \bar{\phi}=1.0, \bar{\phi}=0.9)$. Table 4.1 outlines these experimental flame settings, where the near-stoichiometric flame conditions were identical to those in $[47,128]$. For rich flames under negative $\phi$ gradients $(\delta \phi / \delta y<0)$, the products within the V-flame are from progressively leaner flame regions, such that the flame in the ROI is back supported. Conversely, rich flames with positive $\phi$ gradients $(\delta \phi / \delta y>0)$ are front-supported, as the products are from richer mixtures. Lean flames mirror the behaviour of rich flames such that lean flames with positive $\phi$ gradients $(\delta \phi / \delta y>0)$ are back-supported, as the products are from progressively richer regions. A detailed schematic is provided in Section 4.8.1.

Scalar reactant properties were obtained by seeding iso-octane with 3 -pentanone at $7 \%$ by volume. The associated 3-pentanone PLIF images were processed following the approach outlined in $[47,128]$ and had a signal-to-noise ratio $\left(\mathrm{SNR}=\left(\mu_{\text {fluorescence }}-\mu_{\text {background }}\right) / \sigma_{\text {fluorescence }}\right.$ as defined in [100]) between 5.0-5.4 for $0.95 \leq \phi \leq 1.05$, and $4.4-6.0$ for $0.8 \leq \phi \leq 1.2$. Photon shot noise was characterized by imaging the exit plane of a 4" integrating sphere (SphereOptics LLC) connected to a controllable intensity light source, and ranged between $30.9 \%$ and $14.7 \%$ at $95 \%$ confidence (calculated using photon transfer theory [107]). The location and bounds of the analysis region, or region of interest (ROI), were determined from one thousand instantaneous images acquired along the left-branch of the V-flame (shown in Figure 4.1) at each of three 
axial positions $\mathrm{x}=0-40,40-80$, and $80-120 \mathrm{~mm}$ above the burner for each flame condition. Individual images were ensemble averaged and conditioned on the reactants to obtain reconstructed equivalence ratio maps from $x=0-120 \mathrm{~mm}$. Mean iso-contours of equivalence ratio were then traced to the mean position of the flame front at $\langle\mathrm{c}\rangle=0.5$ to define the location and width of the ROI.

Table 4.1 Summary of test conditions.

\begin{tabular}{|c|c|c|c|c|c|c|}
\hline $\begin{array}{l}\text { iso-contours } \\
\text { of } \phi \text { defining } \\
\text { the ROI }\end{array}$ & $\begin{array}{l}\text { Lewis number } \\
\left(\mathrm{Le}^{*}\right) \text { along iso- } \\
\text { contours of } \phi \\
\text { defining the } \\
\text { ROI }\end{array}$ & $\begin{array}{c}\text { equivalence } \\
\text { ratio } \\
\text { gradient, } \\
\delta \phi / \delta y \\
{\left[\phi \mathrm{mm}^{-1}\right]}\end{array}$ & $\begin{array}{l}\text { width } \\
\text { of ROI } \\
{[\mathrm{mm}]}\end{array}$ & \begin{tabular}{|c|} 
mean $\phi$ of \\
instantaneous \\
images within leaner \\
and richer iso- \\
contours and \\
estimated standard \\
deviation of $\phi$ \\
\end{tabular} & $\begin{array}{c}\text { mean and (standard } \\
\text { deviation } \sigma_{\phi} \text { ) of } \phi \\
\text { along leaner iso- } \\
\text { contour in } \\
\text { instantaneous } \\
\text { images }\end{array}$ & $\begin{array}{c}\text { mean and (standard } \\
\text { deviation } \sigma_{\phi} \text { ) of } \phi \\
\text { along richer iso- } \\
\text { contour in } \\
\text { instantaneous } \\
\text { images }\end{array}$ \\
\hline \multirow{5}{*}{$\begin{array}{c}1.15 \pm 0.023 \\
\text { to } \\
1.25 \pm 0.023\end{array}$} & \multirow{3}{*}{$\mathrm{Le}_{\phi=1.15}=0.70$} & -0.001 & - & $1.204(-)$ & - & - \\
\hline & & 0.005 & 19.3 & $1.202(0.007)$ & $1.150(0.024)$ & $1.248(0.029)$ \\
\hline & & 0.012 & 8.5 & $1.203(0.020)$ & $1.151(0.039)$ & $1.250(0.044)$ \\
\hline & \multirow[t]{2}{*}{$\mathrm{Le}_{\phi=1.25}=0.69$} & -0.007 & 14.1 & $1.196(0.007)$ & $1.148(0.029)$ & $1.148(0.025)$ \\
\hline & & -0.014 & 7.3 & $1.201(0.023)$ & $1.149(0.042)$ & $1.249(0.040)$ \\
\hline \multirow{5}{*}{$\begin{array}{c}0.95 \pm 0.022 \\
\text { to } \\
1.05 \pm 0.023\end{array}$} & \multirow{3}{*}{$\mathrm{Le}_{\phi=0.95}=2.25$} & -0.001 & 12.5 & $1.012(-)$ & - & - \\
\hline & & 0.008 & 12.5 & $0.999(0.008)$ & $0.951(0.029)$ & $1.050(0.031)$ \\
\hline & & 0.015 & 6.7 & $1.000(0.018)$ & $0.949(0.043)$ & $1.049(0.044)$ \\
\hline & \multirow[t]{2}{*}{$\mathrm{Le}_{\phi=1.05}=0.71$} & 0.021 & 4.8 & $1.000(0.024)$ & $0.950(0.061)$ & $1.051(0.061)$ \\
\hline & & 0.029 & 3.5 & $1.001(0.029)$ & $0.952(0.081)$ & $1.051(0.080)$ \\
\hline \multirow{3}{*}{$\begin{array}{c}0.85 \pm 0.022 \\
\text { to } \\
0.95 \pm 0.022\end{array}$} & \multirow[t]{2}{*}{$\overline{\mathrm{Le}_{\phi=0.85}}=2.27$} & -0.001 & - & $0.907(-)$ & - & - \\
\hline & & 0.013 & 7.8 & $0.898(0.019)$ & $0.852(0.038)$ & $0.948(0.044)$ \\
\hline & $\mathrm{Le}_{\phi=0.95}=2.25$ & 0.024 & 4.3 & $0.899(0.028)$ & $0.851(0.064)$ & $0.953(0.075)$ \\
\hline
\end{tabular}

*Lewis numbers calculated from the ratio of thermal to mass diffusivities of the deficient reactant at $55^{\circ} \mathrm{C}$ using simulation data from [128].

Following the approach detailed in [128], the range of local equivalence ratio fluctuations within the ROI was estimated separate from apparent fluctuations due to photon shot noise of the intensified cameras. Standard deviations of $\phi$ within each ROI $\left(\sigma_{\phi}\right)$ were characterized from $\sigma_{\phi}=\sqrt{\sigma_{\text {PLIF }}^{2}-\sigma_{\text {Ref,premixed }}^{2}}$, where $\sigma_{\text {Ref,premixed }}^{2}$ is the statistical variance at equivalent spatial locations for the fully premixed reference case, and $\sigma_{\mathrm{PLIF}}^{2}$ is the observed variance in the raw fluorescence data for each of the stratified cases. The results presented in Table 4.1 show that $\sigma_{\phi}$ increases modestly with $\delta \phi / \delta y$ due to turbulent mixing in the presence of stronger imposed mixture gradients at the exit of the burner. The effects of the varying range of $\phi$ within the ROI of different gradient cases on the ensemble average of the peak HRR and instantaneous flame thickness $\delta_{\mathrm{t}}$ are further discussed in Section 4.4.2. 
Turbulent flow properties were characterized at an axial position $\mathrm{x}=5 \mathrm{~mm}$ above the burner exit plane between transverse positions $y= \pm 20 \mathrm{~mm}$. Measurements were taken under non-reacting conditions with a Dantec LDV system with olive oil seeding. At the mean flow velocity $\bar{U}=5.2 \mathrm{~m} / \mathrm{s}$, the Reynolds number based on the hydraulic diameter of the exit plane was 6120 . The turbulence intensity ranged between $\mathrm{u}^{\prime} / \overline{\mathrm{U}}=3.7-7.7 \%$. Ratios of $\mathrm{u}^{\prime} / \mathrm{S}_{\mathrm{L}}^{\mathrm{o}}$ were calculated based on $\mathrm{S}_{\mathrm{L}, \phi=1.2}^{\mathrm{o}}=36.4 \mathrm{~cm} / \mathrm{s}, \mathrm{S}_{\mathrm{L}, \phi=1.0}^{\mathrm{o}}=$ $37.8 \mathrm{~cm} / \mathrm{s}$, and $\mathrm{S}_{\mathrm{L}, \phi=0.9}^{\mathrm{o}}=33.7 \mathrm{~cm} / \mathrm{s}$, estimated from [95-99] at a reactant temperature of $55^{\circ} \mathrm{C}$, and varied from $0.53-1.10$, to $0.51-1.06$, and $0.57-1.19$ for locally rich, near-stoichiometric, and lean flame regions respectively. Using Taylor's hypothesis, the integral length scale and associated $\operatorname{Re}_{\Lambda}$ were calculated as $\Lambda=5.1 \mathrm{~mm}$ and $\operatorname{Re}_{\Lambda}=82$. The Kolmogorov length scale was calculated to be $\eta=0.19 \mathrm{~mm}$.

\subsection{1 $\mathrm{OH}$ and $\mathrm{CH}_{2} \mathrm{O}$ PLIF Images}

For each flame condition and gradient setting, one thousand pairs of $\mathrm{OH}$ and $\mathrm{CH}_{2} \mathrm{O}$ PLIF images were acquired. Raw fluorescence images were corrected for background intensity and dark current shot noise using standard procedures detailed in [129], as well as shot-to-shot variations in laser intensity and mean laser profile $[47,128]$. Photon shot noise was reduced using $3 \times 3$ median and 7 pixel diameter circular averaging filters for the $\mathrm{OH}$ and $\mathrm{CH}_{2} \mathrm{O}$ images respectively, with typical values ranging between $3.1 \%$ to $4.4 \%\left(\mathrm{SNR}=14.3\right.$, where $\left.\mathrm{SNR}=\left(\mu_{\text {fluorescence }}-\mu_{\text {background }}\right) / \sigma_{\text {fluorescence }}\right)$ and $11.4 \%$ to $20.7 \%(\mathrm{SNR}=5.4$, where $\left.\operatorname{SNR}=\left(\mu_{\text {fluorescence,peak }}-\mu_{\text {background }}\right) / \sigma_{\text {fluorescence,peak }}\right)$ at $95 \%$ confidence.

\subsubsection{Local Peak Heat Release Rate, Flame Thickness, and Curvature}

HRR flame images were obtained following the approach developed by Paul and Najm [55] in which the product of spatially registered, simultaneously acquired, $\mathrm{OH}$ and $\mathrm{CH}_{2} \mathrm{O}$ PLIF images is used to provide a measure of the local heat release rate within the reaction zone of the flame. Detailed numerical simulations of 1D laminar premixed iso-octane/air flames were conducted in [128] to validate the (OH LIF)( $\mathrm{CH}_{2} \mathrm{O}$ LIF) HRR approach for iso-octane/air flames over a broad range of equivalence ratios (where additional simulation runs were performed as part of the present work to extend this range to $\phi=0.65-1.45)$. The resulting HRR contours were studied for local variations in peak HRR and instantaneous flame thickness $\delta_{t}$ by taking individual HRR profiles locally normal to the leading edge of the instantaneous HRR contours, defined using the analysis procedures detailed in [128]. The profiles were extracted at 4 pixel intervals 
$(\sim 0.27 \mathrm{~mm})$ along each instantaneous flame realization. A locally-weighted linear regression filter, or "loess" filter, was applied to the HRR profile data to limit the influence of photon shot noise, and the results were used to determine the local peak HRR intensity and instantaneous flame front thickness $\delta_{\mathrm{t}}$ (measured at full width half maximum, FWHM, of the HRR profiles). Instantaneous measurements of $\delta_{\mathrm{t}}$ were excluded in cases where the SNR was low (11-18\% of data), leaving between 123104 to 26433 individual profiles for analysis for each gradient case. No attempts were made to correct $\delta_{\mathrm{t}}$ for $3 \mathrm{D}$ effects induced by the orientation of the imaging plane relative the flame normal in 3D space, which can lead to an overestimation of the local flame thickness by up to $15 \%$ [130]. Instead, measured values were reported relative to the reference-premixed flame values. Peak HRR and $\delta_{\mathrm{t}}$ were evaluated as a function of the local curvature measured at the same locations along the instantaneous flame contours and were compared among reference-premixed and stratified flame conditions. In addition, variations in the ensemble average of all local peak HRR and retained instantaneous flame thicknesses $\delta_{\mathrm{t}}$ were separately considered.

\subsection{Measurement Uncertainty}

\subsubsection{Local Curvature}

Elemental bias errors in the measurement of two-dimensional curvature $\kappa$ are dominated by the effective spatial resolution of the optical system and the uncertainty in defining the position of a 3D instantaneous flame front of finite thickness within a 2D image. Additional errors can be induced by planar image distortion and magnification. Because the operations involved in the image capture and processing are highly nonlinear and difficult to define using explicit equations, standard approaches to uncertainty estimation using first-order propagation of errors are not suitable. Instead, a Monte Carlo (MC) analysis was used evaluate the contributions of elemental errors to the measurement of local flame curvature.

A MC analysis was performed in which ten instantaneous flame realizations were each processed 5000 times using randomly selected parameters for each required input to the image processing algorithms. Inputs were drawn from pre-determined probability distributions describing the uncertainty or potential variation of that parameter. This process produced 5000 distinct candidate flame contours for each of the ten flame realizations, which were analyzed to quantify measurement uncertainties. During the $\mathrm{MC}$ analysis, the local position of the flame front was altered by randomly varying the chosen threshold values 
and dimensions of the filtering kernel for both the $\mathrm{OH}$ and $\mathrm{CH}_{2} \mathrm{O}$ individual images. This produced variations in local flame position of between 1 and 3 pixels among the candidate flame contours. The leading edge of each candidate contour was then detected. The Cartesian coordinates $x_{i}$ and $y_{i}$ of each data point were then perturbed following a normal distribution $(\sigma=44 \mu \mathrm{m})$, where this prescribed amount of "jitter" was defined by the effective in-plane resolution of the optical system. Figure 4.2a shows three sample sets of flame coordinates (indicated by red, blue, and green circles), from the 5000 sets obtained in the analysis of a sample image. A 12-point cubic spline fit (also shown in Figure 4.2a) was then applied to the jittered coordinates, and local curvature data were calculated at 4 pixel intervals $(\sim 0.27 \mathrm{~mm})$ along each $\mathrm{MC}$ realization at coordinates $\mathrm{x}_{\mathrm{i}, \mathrm{MC}}$ and $\mathrm{y}_{\mathrm{i}, \mathrm{MC}}$. Bias errors induced by the curvature algorithm were evaluated following the approach of Haq et al. [131], by comparing the theoretical and measured curvature of digitized circles. Results showed that any errors from the algorithm were entirely negligible relative to other error sources for the range of curvatures typically observed in experiments. Finally, potential uncertainty in image magnification (normal distribution $\sigma=0.007$ ) and image distortion (normal distribution $\sigma=0.006$ ) were considered in the MC analysis when the outputted local curvature values were converted from pixels ${ }^{-1}$ to $\mathrm{mm}^{-1}$. A summary of inputs and associated uncertainties is included as supplementary material.

Uncertainties in local curvature measurements were quantified by analyzing the distribution of curvature data from the $\mathrm{MC}$ calculations. Because the absolute position of the flame varied among the different $\mathrm{MC}$ draws, care was required in interpreting the MC data. Curvature measurements at locations along the flame front of each $\mathrm{MC}$ realization $\left(\mathrm{x}_{\mathrm{i}, \mathrm{MC}}\right.$ and $\left.\mathrm{y}_{\mathrm{i}, \mathrm{MC}}\right)$ were associated with the nearest location on a reference flame front $\left(\mathrm{x}_{\mathrm{i}, \text { ref }}\right.$ and $\left.\mathrm{y}_{\mathrm{i}, \text { ref }}\right)$, defined as the median location of the flame fronts for all $5000 \mathrm{MC}$ realizations. Curvature distributions could then be calculated at each position along the reference flame front, where the $95 \%$ confidence intervals (CI) in each distribution define the uncertainty in making a local curvature measurement in that image. Figure 4.2a shows sample curvature distributions and associated $95 \%$ confidence intervals calculated at three positions along the flame front.

The local curvature data from all of MC draws were aggregated as a function of the local curvature in the reference image. This allows the results of the uncertainty analysis to be generalized such that overall measurement uncertainty (based on $95 \%$ confidence intervals of the MC data) could be 
expressed as a function of measured curvature. Figure $4.2 \mathrm{~b}$ shows that total measurement uncertainties are very good through the range of curvatures encountered in the present experiments, but grow toward the largest negative curvature values (near $-4 \mathrm{~mm}^{-1}$ ). However, uncertainties at these large curvature values are dominated by potential bias errors associated with choice of parameters in the image processing algorithms. By repeating the $\mathrm{MC}$ analysis and fixing parameters that remained constant through all experiments (i.e. the threshold, filter kernel, planar image distortion, and magnification terms), it was possible to separately estimate the relative uncertainty in making comparative measurements (i.e. uncertainty when comparing curvature variations among different equivalence ratio gradient cases). Figure $4.2 \mathrm{~b}$ also shows that the relative uncertainties are much tighter, especially at the largest negative curvature values, and provide quantitative support for the comparisons presented with the results.
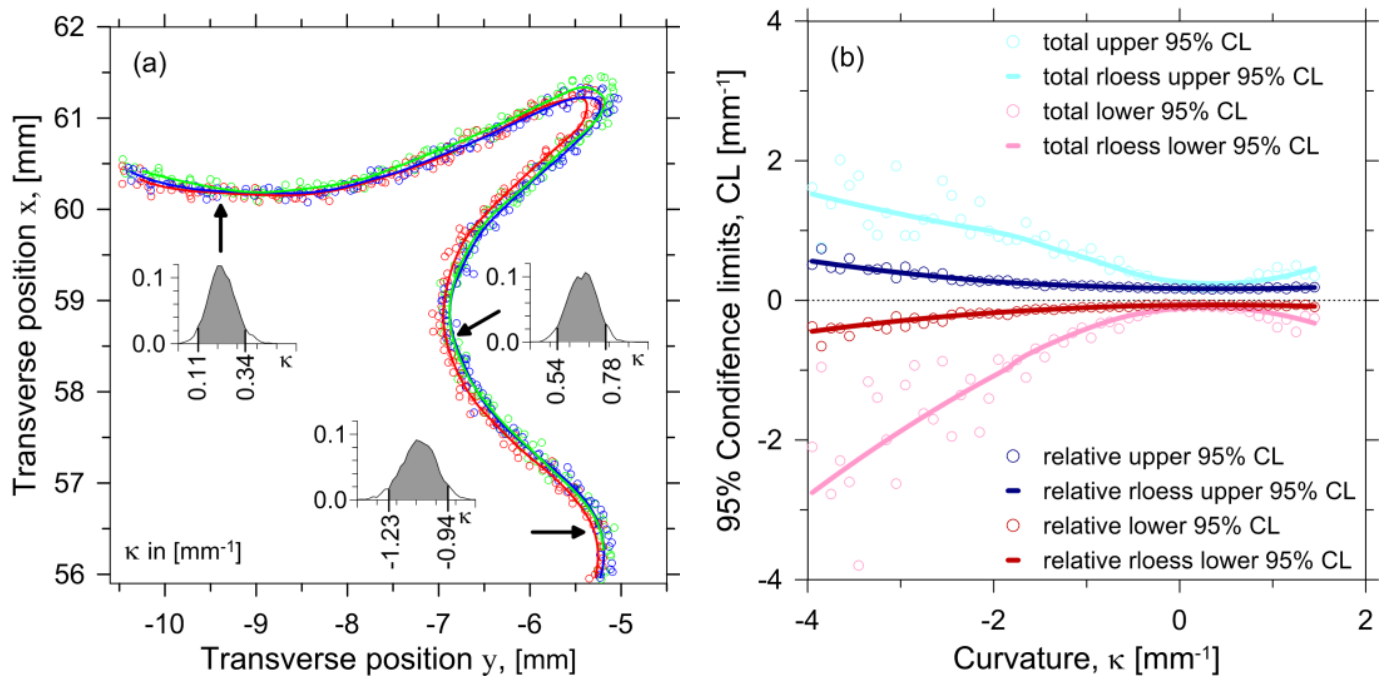

Figure 4.2 Quantification of curvature uncertainty. In (a) open circles show spatially jittered flame positions for three sample runs of the Monte Carlo analysis, while sample histograms show distributions of local curvature and $95 \%$ confidence limits evaluated at three different sample positions along the flame. Final computed $95 \%$ confidence limits for total and relative curvature uncertainty in (b).

\subsubsection{Local Peak Heat Release Rate and Flame Thickness}

The uncertainty of the local heat release rate along a stratified flame front is primarily dependent on the elemental errors of $\mathrm{OH}$ and $\mathrm{CH}_{2} \mathrm{O}$ fluorescence signals. These are a function of spectroscopic properties unique to both species (i.e. temperature induced variations in Boltzmann population fractions and collisional quenching factors specific to the excitation transitions being used), as well as the systematic 
errors of the apparatus (i.e. photon shot noise of the intensified CCDs, pulse-to-pulse variations in local laser fluence, and fluorescence trapping). These elemental errors are discussed in the context of estimating the uncertainty of relative variations in local peak HRR and instantaneous flame thickness $\delta_{\mathrm{t}}$ among conditions.

\subsubsection{Relative Variations in Peak HRR and Flame Thickness with Curvature}

In Figure 4.5, local measurements of peak HRR and instantaneous flame thickness are plotted as a function of local curvature. Data are binned in intervals of curvature $\kappa=0.1 \mathrm{~mm}^{-1}$, and the ensemble average of each parameter is determined within each curvature bin. The resulting mean data are then normalized by the corresponding mean HRR value at curvature $\kappa=0 \mathrm{~mm}^{-1}$ for each flame setting to emphasize their relative variation with curvature.

The contribution of systematic errors to the relative uncertainties of the local peak HRR and instantaneous flame thickness $\delta_{\mathrm{t}}$ were evaluated via MC simulation similar to that of relative curvature. The same ten images were processed 5000 times by fixing inputs to the image processing routine that remained constant among flame settings (threshold, filter kernel, and image distortion and magnification). The spatial coordinates of the flame front were then "jittered" before individual $\mathrm{OH}$ and $\mathrm{CH}_{2} \mathrm{O}$ profiles were extracted normal to the resulting instantaneous flame front. The individual pixel values of both species were then varied based on the expected photon shot noise at the corresponding pixel count specific to each ICCD (determined from white field images acquired using a 4" integrating sphere (SphereOptics LLC) and calculated using photon transfer theory [107]). Gaussian distributions were generated based on the local pixel count and ICCD gain such that uncertainties ranged from $28 \%-3 \%$ in $\mathrm{OH}$ and $43 \%-11 \%$ in $\mathrm{CH}_{2} \mathrm{O}$ (corresponding to the minimum and maximum observed fluorescence intensities respectively) at 95\% confidence. The resulting "noisy" $\mathrm{OH}$ and $\mathrm{CH}_{2} \mathrm{O}$ profiles were then multiplied to obtain individual HRR profiles. Elemental errors induced by the pulse-to-pulse laser fluctuations were included by multiplying the individual HRR profiles by values drawn from a normal distribution based on the associated uncertainties in each laser sheet. Uncertainties due to pulse-to-pulse variations in laser profile were $6.3 \%$ and $8.2 \%$ at $95 \%$ confidence for the $283 \mathrm{~nm}$ and $355 \mathrm{~nm}$ beams respectively. Laser energy corrections were accurate to within $0.05 \%$ for both beams and therefore omitted from the MC. A loess 
filter was then applied to the adjusted HRR profiles and the outputted peak and FWHM thickness calculated. Additional contributions to the uncertainty such as image registration, laser absorption and scattering, background signal, fluorescence trapping, line shape, and measurement volume were either corrected for during measurements or assumed constant among experiments, and therefore not expected to contribute to the uncertainty of relative HRR measurements.

In our previous work [128], an analysis of elemental errors affecting the spectroscopic properties of $\mathrm{OH}$ and $\mathrm{CH}_{2} \mathrm{O}$ confirmed that for the excitation transitions used, the pixel-by-pixel product of raw $(\mathrm{OH} \mathrm{LIF})\left(\mathrm{CH}_{2} \mathrm{O}\right.$ LIF) signals is an excellent marker for relative HRR measurements in premixed isooctane/air flames. Results of flame simulations showed that this was true over a broad range of equivalence ratios. Although local measurements of instantaneous HRR along a stratified flame would require spatially resolved temperature information (to account for variations in Boltzmann population fractions and collisional quenching rates), bias errors in the ensemble averaged HRR could still be corrected, given information on the range of equivalence ratios within the ROI. This result can be directly applied to the data presented in Figure 4.5, since it is expected that the range of equivalence ratios within each curvature bin remains constant for a given flame setting. This implies that the potential bias error of each bin would be consistent, and therefore not affect the relative shape of the normalized curves shown in Figure 4.5 .

To estimate the final confidence intervals of a given peak HRR and FWHM flame thickness $\left(\delta_{\mathrm{t}}\right)$ measurement, local data from each $\mathrm{MC}$ draw were binned as a function of the nearest data point $\left(\mathrm{x}_{\mathrm{i}, \mathrm{ref}}\right.$ and $\mathrm{y}_{\mathrm{i}, \mathrm{ref}}$ ) along the reference image. The $95 \%$ confidence intervals at each reference location were then determined for both the peak and FWHM of the HRR profiles as plotted in Figure 4.3. Empty circles represent the relative error for values of peak HRR and flame thickness $\delta_{t}$, while the lines represent the corresponding robust loess (rloess) fit to these data. Figure 4.3 a reveals that the $95 \%$ confidence limits are approximately level over the range of peak HRR values expected for a typical data set, with the upper and lower bounds generally on the order of $\sim+12 \%$ and $-7 \%$. The confidence limits of $\delta_{t}$ in Figure $4.3 b$ oscillate slightly, but remain within $\sim+11 \%$ and $-8 \%$. 

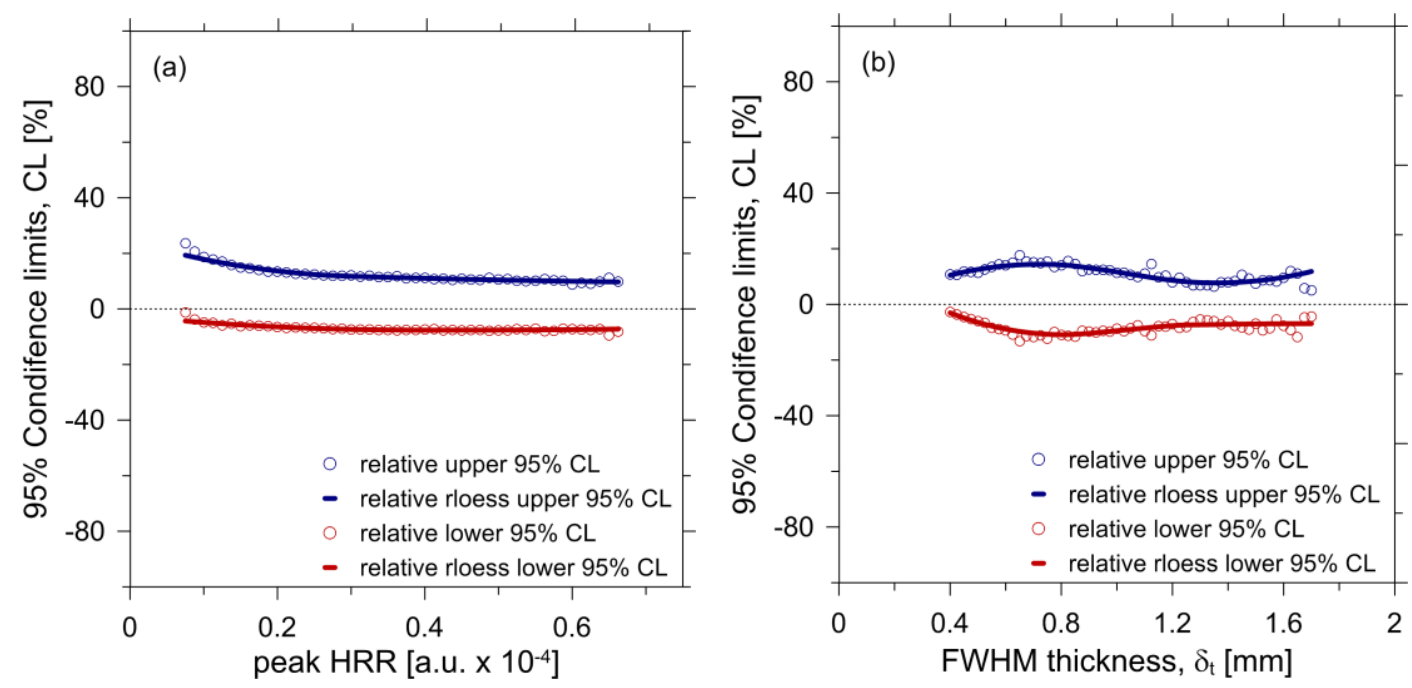

Figure $4.395 \%$ confidence intervals for the relative uncertainty of the local peak HRR (a) and the local flame thickness $\delta_{t}(b)$.

\subsubsection{Ensemble Average of Peak HRR and Flame Thickness}

Table 4.3 shows potential bias errors on the mean peak HRR and $\delta_{t}$ which were quantified following the approach described in [128]. Briefly, a MC analysis was used to consider variations in the range of equivalence ratios within each ROI among gradient settings due to turbulent mixing of the imposed $\phi$ gradient at the exit of the burner. These variations are characterized from the $\sigma_{\phi}$ values reported in Table 4.1. Twenty thousand synthetic distributions of $\phi$ within the ROI were generated for each gradient condition by taking spatially resolved $\sigma_{\phi}$ data along $\phi$ iso-contours between the leaner and richer isocontours in steps of $\phi=0.01$. These data were then coupled with the results of simulated HRR data for premixed flames, and the expected temperature dependence of $\mathrm{OH}$ and $\mathrm{CH}_{2} \mathrm{O}$ fluorescence signals (calculated from [94,117-119] and based on the Boltzmann population fractions and collisional quenching rates of both species [94,117-119]). By deriving correlation functions relating the local equivalence ratio to the expected peak and FWHM thickness of simulated (OH LIF)( $\mathrm{CH}_{2} \mathrm{O}$ LIF) profiles, corresponding synthetic distributions of peak HRR and flame thickness $\delta_{\mathrm{t}}$ could be calculated, and the resulting bias error relative to the reference-premixed case determined. Anticipated error values are summarized in Table 4.2 and are expressed in terms of the ratio of the stratified to the corresponding reference-premixed case. This extended analysis allows the potential effects of mean gradients in equivalence ratio on local flame 
properties to be isolated from effects attributable to variations in local equivalence ratio and the elemental errors of (OH LIF) $\left(\mathrm{CH}_{2} \mathrm{O}\right.$ LIF) within the ROI.

Table 4.2 Bias error of ensemble average of local peak HRR and instantaneous flame thickness $\delta_{t}$ due to combined effects of the differing range of $\phi$ within the ROI and the temperature dependence of the $\mathrm{OH}$ and $\mathrm{CH}_{2} \mathrm{O}$ fluorescence signals.

\begin{tabular}{|c|c|c|c|c|c|c|c|c|c|c|}
\hline $\begin{array}{l}\text { iso-contours of } \phi \\
\text { defining the ROI }\end{array}$ & \multicolumn{4}{|c|}{$1.15 \leq \phi \leq 1.25$} & \multicolumn{4}{|c|}{$0.95 \leq \phi \leq 1.05$} & \multicolumn{2}{|c|}{$0.85 \leq \phi \leq 0.95$} \\
\hline $\begin{array}{c}\text { equivalence ratio } \\
\text { gradient, } \delta \phi / \delta y \\
{\left[\phi \mathrm{mm}^{-1}\right]}\end{array}$ & 0.005 & 0.012 & -0.007 & -0.014 & 0.008 & 0.015 & 0.021 & 0.029 & 0.013 & 0.024 \\
\hline $\begin{array}{c}\mathrm{HRR}_{\text {peak }} \text { bias error } \\
{[\%]}\end{array}$ & $\begin{array}{l}+1.8 \text { to } \\
+1.7\end{array}$ & $\begin{array}{l}+1.3 \text { to } \\
+1.1\end{array}$ & $\begin{array}{l}+1.8 \text { to } \\
+1.6\end{array}$ & $\begin{array}{c}+1.2 \text { to } \\
+0.9\end{array}$ & $\begin{array}{c}-1.0 \text { to } \\
-1.1\end{array}$ & $\begin{array}{c}-1.8 \text { to } \\
-2.0\end{array}$ & $\begin{array}{c}-3.2 \text { to }- \\
3.4\end{array}$ & $\begin{array}{c}-5.3 \text { to }- \\
5.5\end{array}$ & $\begin{array}{c}-2.6 \text { to }- \\
2.6\end{array}$ & $\begin{array}{c}-4.5 \text { to }- \\
4.5\end{array}$ \\
\hline $\begin{array}{c}\delta_{\mathrm{t}} \text { bias error } \\
{[\%]}\end{array}$ & $\begin{array}{l}+0.2 \text { to } \\
+0.3\end{array}$ & $\begin{array}{c}+1.0 \text { to } \\
+1.1\end{array}$ & $\begin{array}{c}+0.3 \text { to } \\
+0.3\end{array}$ & $\begin{array}{c}+1.0 \text { to } \\
+1.1\end{array}$ & $\begin{array}{l}+0.5 \text { to } \\
+0.5\end{array}$ & $\begin{array}{l}+0.8 \text { to } \\
+0.8\end{array}$ & $\begin{array}{l}+1.4 \text { to } \\
+1.4\end{array}$ & $\begin{array}{l}+2.2 \text { to } \\
+2.3\end{array}$ & $\begin{array}{l}+0.8 \text { to } \\
+0.9\end{array}$ & $\begin{array}{c}+1.7 \text { to } \\
+1.8\end{array}$ \\
\hline
\end{tabular}

\subsection{Results and Discussion}

The effects of equivalence ratio gradient on locally rich, near-stoichiometric, and lean flame regions were quantified by analyzing HRR profiles taken normal to the leading edge of instantaneous HRR contours. The profiles were smoothed with a loess filter and analyzed for variations in local peak HRR and $\delta_{\mathrm{t}}$ among $\phi$ gradient conditions for each of the three global mixture strength cases. Figure 4.4 shows a set of sample normalized profiles of (OH LIF), $\left(\mathrm{CH}_{2} \mathrm{O}\right.$ LIF), and $(\mathrm{OH}$ LIF $)\left(\mathrm{CH}_{2} \mathrm{O}\right.$ LIF) HRR at a single position along a single instantaneous flame front for globally rich, stratified flame setting $\delta \phi / \delta y=0.012 \mathrm{~mm}^{-1}$. Data are centered at the location of maximum HRR, which roughly corresponds with the intersection of the (OH LIF) and $\left(\mathrm{CH}_{2} \mathrm{O}\right.$ LIF) plots. The profiles in Figure 4.4 are comparable to the experimental results of [57,61], and simulations of $[56,128]$, with the Gaussian-like $\mathrm{CH}_{2} \mathrm{O}$ profile positioned slightly upstream of the error-function-like $\mathrm{OH}$ profile. The HRR profiles resemble a Gaussian, with a slightly elevated tail on the product side, as discernable in the sample case shown in Figure 4.4. This is caused by the low signal to noise ratio of the $\left(\mathrm{CH}_{2} \mathrm{O}\right.$ LIF) images relative to that of (OH LIF), as the residual background noise in the $\left(\mathrm{CH}_{2} \mathrm{O}\right.$ LIF) images was multiplied by the significantly higher (OH LIF) signal within the products. 


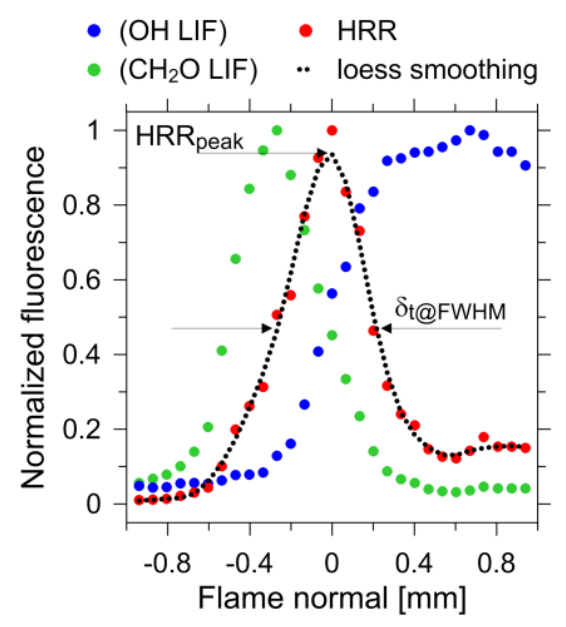

Figure 4.4 Example normalized profiles of (OH LIF) and $\left(\mathrm{CH}_{2} \mathrm{O}\right.$ LIF) fluorescence intensity and $(\mathrm{OH} \mathrm{LIF})\left(\mathrm{CH}_{2} \mathrm{O}\right.$ LIF $)$ HRR at a position along an instantaneous realization of the globally rich, stratified flame condition at $\delta \phi / \delta y=0.012 \mathrm{~mm}^{-1}$.

The effects of equivalence ratio gradient were first evaluated by plotting the local instantaneous peak HRR obtained from the loess-smoothed profiles as a function of curvature. Data were binned at intervals of curvature $\kappa=0.1 \mathrm{~mm}^{-1}$, and the mean peak HRR value within each bin was normalized by the mean peak HRR value at curvature $\kappa=0 \mathrm{~mm}^{-1}$. The resulting curves emphasize variations in the distribution of peak HRR with curvature among gradient conditions and are shown in Figure 4.5 (a), (b), and (c) for locally rich, near-stoichiometric, and lean flame regions respectively.

Initial comparison of fully premixed reference cases in (a), (b), and (c) showed significant differences in the distribution of peak HRR with curvature, and the results were consistent with that expected based on visual inspection of individual flame images. As anticipated by thermodiffusive theory $[19,81]$, the general distributions of the reference-premixed cases reflect the influence of competing effects of mass and thermal diffusion. For locally rich flame regions with $\mathrm{Le}<1$, lower diffusive flux of the deficient reactant leads to relatively lower HRR in negatively curved (concave toward the reactants) flame regions, while HRR is relatively higher in positively curved regions where the diffusive flux is enhanced. Conversely, the distributions of near-stoichiometric and lean $(\operatorname{Le}>1)$ flame regions show a gradual, relative increase in peak HRR in negative curvature areas, as the incoming reactants to concave flame regions are further pre-heated by the flame front due to greater thermal diffusion. The lean premixed 
profiles in Figure 4.5c are similar to those reported for lean ethylene/air flames with Le $>1[57,132]$, where the authors observed greater HRR in negatively curved flame regions.

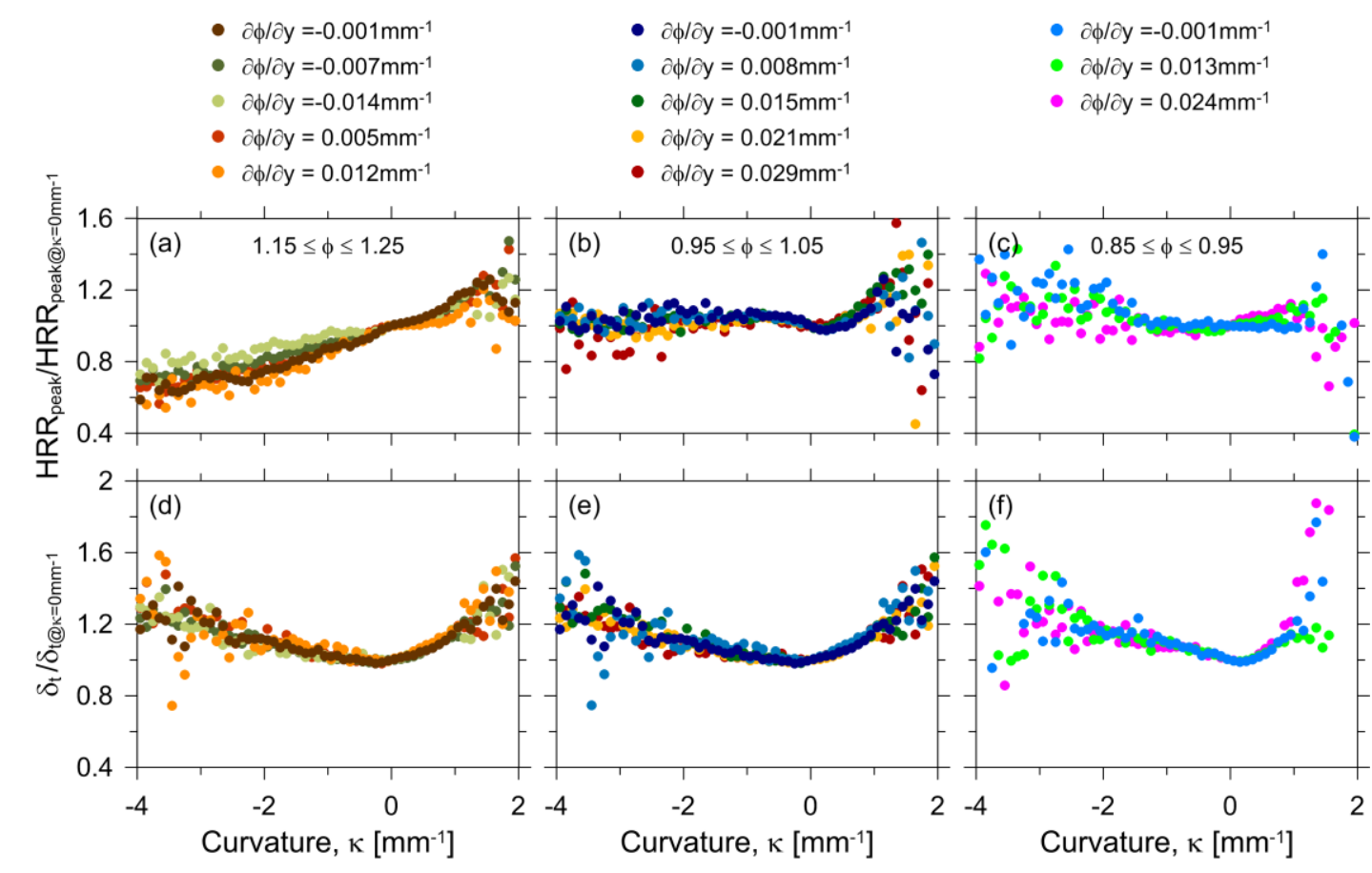

Figure 4.5 Mean HRR and instantaneous flame thickness $\delta_{t}$ vs curvature for (a,d) rich front- and back-supported, $(b, e)$ near-stoichiometric, and $(c, f)$ lean back-supported premixed and stratified flame conditions.

Within the three main mixture strength conditions shown in Figure 4.5 (a), (b), and (c), the differences in trends among gradient conditions were generally small. Although slightly more evident for the locally rich and lean flames than for the near-stoichiometric flames at comparable gradients, these changes are much less pronounced than the variations in ensemble averaged peak HRR (i.e. the mean peak HRR for all curvatures) with gradient presented in Table 4.3.

The peak HRR of locally rich, back-supported flames (negative $\phi$ gradients $\delta \phi / \delta y=-0.007 \mathrm{~mm}^{-1}$ and $\delta \phi / \delta y=-0.014 \mathrm{~mm}^{-1}$ ) gradually increased in regions of negative curvature, while negligible variation occurred in positive curvatures. Conversely, locally rich, front-supported flames (positive gradients $\delta \phi / \delta \mathrm{y}=0.005 \mathrm{~mm}^{-1}$ and $\delta \phi / \delta \mathrm{y}=0.012 \mathrm{~mm}^{-1}$ ) resulted in a negligible change in negative curvatures, while a small decrease was observed in positive curvatures. Though minimal variations were observed in the nearstoichiometric flame regions in (b), back-supported, lean flame regions in (c) (positive gradients 
$\delta \phi / \delta \mathrm{y}=0.013 \mathrm{~mm}^{-1}$ and $\delta \phi / \delta \mathrm{y}=0.024 \mathrm{~mm}^{-1}$ ) showed a slight decrease in the peak HRR in areas of negative curvature, coupled with an increase in peak HRR in areas of positive curvature.

While the current data do not speak to the specific physical mechanisms that may alter local flame behaviour, the observed small differences among gradient conditions do provide evidence of the potential interaction between neighbouring flame regions. They suggest that stratification may lead to small differences in local peak HRR distribution with curvature, and potentially influence the local thermodiffusive stability of the wrinkled structures along a turbulent flame front. This would also explain the comparatively negligible changes observed for near-stoichiometric flames in Figure 4.5b, where thermodiffusive effects are irrelevant.

Figure 4.5 (d), (e), and (f) plot the distribution in local instantaneous flame thickness $\delta_{\mathrm{t}}$ with curvature for all flame conditions. Data were binned at intervals of curvature of $\kappa=0.1 \mathrm{~mm}^{-1}$ as with the peak HRR data. General distributions remained similar for the three main flame categories, and results were comparable to those of Robin et al. [29] for turbulent methane/air premixed and stratified V-flames, who reported a significant increase in local thermal flame thickness in areas of positive and negative curvature. No discernible changes were observed between premixed and stratified flames in any of the three main cases despite the small changes in peak HRR with curvature.

Table 4.3 summarizes the ensemble average of all local peak HRR values and instantaneous flame thicknesses $\delta_{t}$ of the smoothed profiles considered in Figure 4.5. The measured values of gradient cases are expressed in terms of their ratio to the corresponding reference-premixed flame condition. Estimated standard deviations, $\sigma_{\text {est }}$, are also included and were calculated as the square root of the difference between the measured variances of the reference premixed and stratified flame settings. Initial observations revealed that local peak HRR and flame thickness $\delta_{t}$ had generally opposing trends, such that an increase in peak HRR was correlated with a thinning of the profiles and vice versa. This was observed for all flame settings. 
Table 4.3 Peak HRR fluorescence intensity and FWHM profile thickness $\delta_{t}$.

\begin{tabular}{|c|c|c|c|c|c|c|c|c|c|c|}
\hline $\begin{array}{l}\text { iso-contours of } \phi \\
\text { defining the ROI }\end{array}$ & \multicolumn{4}{|c|}{$1.15 \leq \phi \leq 1.25$} & \multicolumn{4}{|c|}{$0.95 \leq \phi \leq 1.05$} & \multicolumn{2}{|c|}{$0.85 \leq \phi \leq 0.95$} \\
\hline $\begin{array}{c}\text { gradient, } \delta \phi / \delta y \\
{\left[\phi \mathrm{mm}^{-1}\right]}\end{array}$ & 0.005 & 0.012 & -0.007 & -0.014 & 0.008 & 0.015 & 0.021 & 0.029 & 0.013 & 0.024 \\
\hline$\frac{\text { HRR }_{\text {peak,strat }}{ }^{*}}{\mathrm{HRR}_{\text {peak,ref }}} \pm \sigma_{\text {est }}$ & $\begin{array}{c}0.954- \\
0.955 \\
\pm 0.055\end{array}$ & $\begin{array}{c}0.947- \\
0.949 \\
\pm 0.065\end{array}$ & $\begin{array}{c}1.060- \\
1.062 \\
\pm 0.043\end{array}$ & $\begin{array}{c}1.099- \\
1.102 \\
\pm 0.060\end{array}$ & $\begin{array}{c}0.982- \\
0.983 \\
\pm 0.053\end{array}$ & $\begin{array}{c}0.993- \\
0.995 \\
\pm 0.066\end{array}$ & $\begin{array}{c}0.968- \\
0.970 \\
\pm 0.074\end{array}$ & $\begin{array}{c}0.965- \\
0.967 \\
\pm 0.088\end{array}$ & $\begin{array}{c}1.027- \\
1.027 \\
\pm 0.068\end{array}$ & $\begin{array}{c}1.038- \\
1.038 \\
\pm 0.083\end{array}$ \\
\hline$\frac{\delta_{\mathrm{t}, \mathrm{strat}}{ }^{*}}{\delta_{\mathrm{t}, \mathrm{ref}}} \pm \sigma_{\mathrm{est}}$ & $\begin{array}{c}1.014- \\
1.015 \\
\pm 0.042\end{array}$ & $\begin{array}{c}1.023- \\
1.024 \\
\pm 0.065\end{array}$ & $\begin{array}{c}0.938- \\
0.938 \\
\pm 0.046\end{array}$ & $\begin{array}{c}0.928- \\
0.929 \\
\pm 0.058\end{array}$ & $\begin{array}{c}1.009- \\
1.009 \\
\pm 0.041\end{array}$ & $\begin{array}{c}1.012- \\
1.012 \\
\pm 0.051\end{array}$ & $\begin{array}{c}1.019- \\
1.019 \\
\pm 0.069\end{array}$ & $\begin{array}{c}1.027- \\
1.028 \\
\pm 0.074\end{array}$ & $\begin{array}{c}0.988- \\
0.989 \\
\pm 0.054\end{array}$ & $\begin{array}{c}0.979- \\
0.980 \\
\pm 0.075\end{array}$ \\
\hline
\end{tabular}

Differences in magnitude reported in Table 4.3 were significantly more pronounced than the relative changes in distribution in Figure 4.5. Flame regions showed opposite trends in peak HRR between front- and back-supported gradient conditions. Decreases in peak HRR and corresponding increases in $\delta_{\mathrm{t}}$ were observed for front-supported, locally rich flame regions, whereas increases in peak HRR and corresponding decreases in $\delta_{\mathrm{t}}$ were observed in back-supported locally rich flame regions. Nearstoichiometric flame regions showed a general decrease in peak HRR and increase in $\delta_{\mathrm{t}}$ as the positive $(\delta \phi / \delta y>0)$ mean $\phi$ gradient steepened, though the effect was less pronounced for equivalent gradient levels.

Back-supported, locally lean flame regions showed an increase in peak HRR and decrease in $\delta_{t}$ with gradient, consistent with the simulations of Richardson et al. [72] on lean $\mathrm{CH}_{4} /$ air counterflow flames. Trends were generally analogous to those of back-supported rich flame regions, though differences were significantly less pronounced at equivalent gradient levels, as only slight increases in mean peak HRR were observed. This difference in effect may simply be because the locally lean flame regions $(\bar{\phi}=0.9)$ are closer to stoichiometric $(\bar{\phi}=1.0)$, where the effects appear least significant. However, because the combustion intensity peaks at $\bar{\phi} \approx 1.07$, the lean reactant mixtures are actually weaker $\left(\mathrm{S}_{\mathrm{L}, \phi=0.9}^{o}=\right.$ $\left.33.7 \mathrm{~cm} / \mathrm{s}[95-99], \mathrm{HRR}_{\text {peak }, \phi=0.9} / \mathrm{HRR}_{\text {peak }, \phi=1.0} \approx 0.82\right)$ than the comparison locally rich $(\bar{\phi}=1.2)$ flame regions $\left(\mathrm{S}_{\mathrm{L}, \phi=1.2}^{\circ}=36.4 \mathrm{~cm} / \mathrm{s}[95-99], \mathrm{HRR}_{\text {peak }, \phi=1.2} / \mathrm{HRR}_{\mathrm{peak}, \phi=1.0} \approx 0.85\right)$. In this context, the results further suggest that the primary physical mechanisms of back support for rich flames (species) and lean flames (heat) $[26,80]$ differ with local stoichiometry. 
The normalized distributions of the local instantaneous peak HRR and flame thickness $\delta_{t}$ in Figure 4.5 and the corresponding ensemble averaged values in Table 4.3, suggest that back support of the heated products to the leading stratified branches is important in the context of V-flames. Small changes in the distribution of peak HRR with curvature of flames within the ROI were observed among gradient cases, suggesting that stratification may impact the local thermodiffusive stability of the flame front. Differences were more pronounced for the ensemble averaged HRR data over all curvatures in Table 4.3, implying that $\phi$ gradients have a more significant effect on the relative magnitudes of peak HRR and $\delta_{t}$ than on their distribution with curvature. Overall, these results suggest that gradient effects on the leading flame front are non-negligible in stratified V-flames, even at the relatively modest gradients considered in this study.

\subsection{Conclusion}

The effects of stratification on locally rich, near-stoichiometric, and lean flame regions were evaluated in stratified iso-octane/air, turbulent V-flames. Instantaneous heat release rate measurements were obtained from the pixel-by-pixel product of spatially registered, near-simultaneously acquired $\mathrm{OH}$ and $\mathrm{CH}_{2} \mathrm{O}$ PLIF images. Individual HRR flame contours were studied by extracting intensity profiles normal to their leading edge and analyzing for variations in local peak HRR and instantaneous flame thickness $\delta_{t}$. Gradient flame settings were compared to reference-premixed flame settings by analyzing HRR data strictly within ROIs specific to each gradient. This ensured a constant mean range of equivalence ratios was considered among flame conditions, effectively isolating the effects of mean equivalence ratio gradient from those of local mixture strength.

Evidence of the interaction between neighbouring flame regions was observed as plots of peak HRR with curvature varied among gradient conditions in Figure 4.5. Differences were less pronounced for near-stoichiometric flame regions than for locally rich and lean flame regions. These results suggested that stratification may influence the local thermodiffusive stability of the turbulent flame front in these isooctane/air flames.

Small yet repeatable variations in the ensemble average of all local peak HRR and instantaneous

flame thicknesses $\delta_{\mathrm{t}}$ were observed when comparing stratified and reference-premixed flame conditions in Table 4.3. Front- $(\delta \phi / \delta y>0)$ and back- $(\delta \phi / \delta y<0)$ supported rich flames showed opposing trends for both 
peak HRR and $\delta_{\mathrm{t}}$, as back-supported flames resulted in an increased peak HRR and narrowing of $\delta_{\mathrm{t}}$, while front-supported flames lead to a decrease in peak HRR and a thickening of $\delta_{\mathrm{t}}$. Near-stoichiometric flame regions were also affected by stratification as a decrease in peak HRR and a broadening of $\delta_{t}$ was observed with increasing gradient, though not as significantly as the locally rich flame regions for equivalent gradient levels. Trends for back-supported $(\delta \phi / \delta y>0)$ lean flame regions were generally analogous to those of back-supported rich flame regions, although the increase in peak HRR was less pronounced relative to the reference-premixed case. These data suggest that back-support becomes less important for flames nearer to stoichiometric, and further imply that different mechanisms of back-support may occur between rich and lean flames, in line with the numerical predictions of $[26,80]$.

Overall, these results indicate that mean gradients of equivalence ratio induce non-negligible interaction between neighbouring flame regions in stratified, turbulent iso-octane/air V-flames. The subtle differences in the local distribution of peak HRR with curvature, and more pronounced variations in the magnitude of the mean local peak HRR and instantaneous flame thickness $\delta_{t}$ provide evidence of backsupport from the heated products to the leading reaction zone. While reported trends were small, it is important to note that these variations were observed for flame regions in which the mean local stoichiometry was held constant, and were separate from potential effects of differences in local mixture strength. Furthermore, the current study was limited to relatively weak spatial gradients in equivalence ratio, such that steeper $\phi$ gradients observed in stratified charge engines and gas turbines are expected to influence flame behaviour much more significantly. It is therefore necessary to further the fundamental understanding of potential gradient effects as a pre-requisite to the continued optimization and development of next generation, internal combustion devices.

\subsection{Acknowledgements}

The authors are indebted to Greg Smallwood (National Research Council of Canada) for providing access to laboratory facilities and support of the experiments, as well as Hongsheng Guo (National Research Council of Canada) who provided simulation data used to validate the experimental methodology for heat release rate measurements. 


\subsection{Supplemental Material}

\subsubsection{Further Explanation of "Front-Supported" and "Back-Supported" Flame Configurations}

The concept of front- and back-supported flames was introduced by Marzouk et al. [70] in the context of a planar flame propagating solely in the direction of the gradient vector of $\phi$. Flames propagating toward progressively weaker mixtures are described as "back-supported" by the downstream products of stronger reactant mixtures. By contrast, flames burning toward stronger mixtures, are described as "frontsupported" by the stronger upstream reactants. In V-flames, the flame front is obliquely oriented relative to the mean $\phi$ gradient, such that a gradient is imposed in both the axial and transverse direction along the stratified reaction zone. Consistent with the convention in the literature [31], we have defined a "backsupported" configuration, shown in Figure 4.6a and Figure 4.6c for locally rich and lean flame regions respectively, as a flame where the products within the $\mathrm{V}$-flame, and adjacent to the ROI, are a result of flame regions burning in stronger reactant mixtures. "Front-supported" flames, shown in Figure 4.6b for locally rich flame regions, are defined as the case where the products within the V-flame and adjacent to the ROI are weaker.
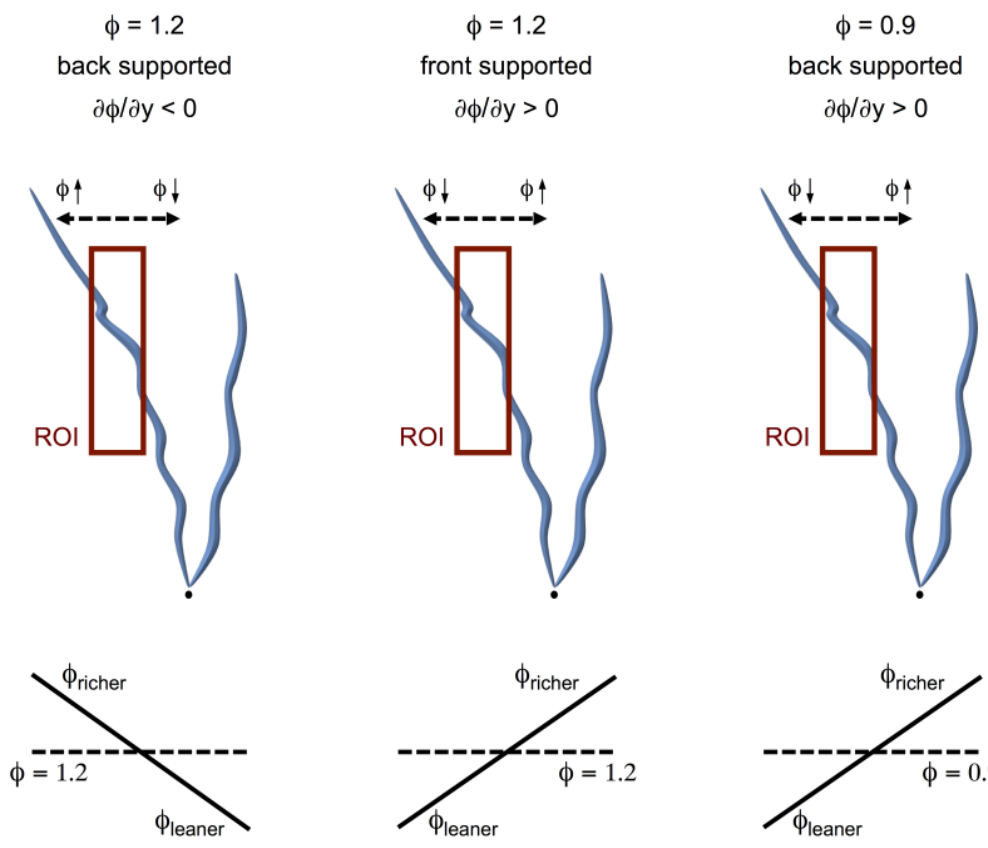

Figure 4.6 Schematic highlighting the variation in $\phi$ for flame regions adjacent to the ROI for "front-supported" and "back-supported" V-flames. 


\subsubsection{Details on the Inputs of the Monte Carlo Uncertainty Simulations of Local Curvature, Peak HRR, and FWHM Flame Thickness}

Table 4.4 Summary of input distributions used for the Monte Carlo simulations of the uncertainty of local curvature, peak HRR, and FWHM flames thickness along an instantaneous flame realization.

\begin{tabular}{|c|c|c|c|c|}
\hline Variable & Distribution & Parameters & & Units \\
\hline \multicolumn{5}{|l|}{ Variables used for Curvature } \\
\hline $\mathrm{OH}$ filtering kernel & Random & $\mu: 3 \times 3$ & $\min / \max =2 \times 2 / 4 \times 4$ & pixels \\
\hline OH threshold & Random & $\mu=0.085$ & $\min / \max =0.045 / 0.125$ & a.u. \\
\hline $\mathrm{CH}_{2} \mathrm{O}$ filtering kernel & Random & $\mu: D=7$ & $\min / \max =5 / 9$ & pixels \\
\hline $\mathrm{CH}_{2} \mathrm{O}$ threshold & Random & $\mu=0.0010$ & $\min / \max =0.0008 / 0.0012$ & a.u. \\
\hline jitter of spatial coordinates & Normal & $\mu=0$ & $\sigma=44$ & $\mu \mathrm{m}$ \\
\hline image magnification & Normal & $\mu=1$ & $\sigma=0.007$ & {$[-]$} \\
\hline image distortion & Normal & $\mu=1$ & $\sigma=0.006$ & {$[-]$} \\
\hline \multicolumn{5}{|c|}{ Additional variables used for HRR profile peak and FWHM thickness } \\
\hline $283 \mathrm{~nm}$ laser sheet profile & Normal & $\mu=1$ & $\sigma=0.032$ & a.u. \\
\hline $355 \mathrm{~nm}$ laser sheet profile & Normal & $\mu=1$ & $\sigma=0.042$ & a.u. \\
\hline OH photon shot noise & Normal & $\mu=1$ & $\sigma_{\min }=0.015, \sigma_{\max }=0.143$ & a.u. \\
\hline $\mathrm{CH}_{2} \mathrm{O}$ photon shot noise & Normal & $\mu=1$ & $\sigma_{\min }=0.056, \sigma_{\max }=0.219$ & a.u. \\
\hline
\end{tabular}




\section{Chapter 5}

\section{Conclusions and Recommendations}

\subsection{Conclusions}

This thesis has made several contributions to the study of turbulent stratified flames, and more specifically to the investigation of the interaction between neighbouring flame regions in the presence of a mean gradient in equivalence ratio. The work presented initially focused on developing an experimental apparatus and methodology capable of isolating the specific effects of mean $\phi$ gradients from effects attributable to varied mixture strength, and evaluating their influence on local flame properties. Coupled with a novel rectangular exit burner that permitted controlled, transverse variations in $\phi$ along its exit, a unique variable windowing technique was used to define an analysis region of interest and study locally rich, near-stoichiometric, and lean stratified, iso-octane/air turbulent V-flames for variations in local flame topology and heat release rate. While several experimental studies have presented data on the overall behaviour of partially premixed flames, to the author's knowledge, the work presented in this thesis was the first experimental investigation of the effects of mean $\phi$ gradients in turbulent iso-octane/air flames.

The analysis region of interest (ROI) specific to each gradient flame setting was obtained from the conditioned, ensemble average of instantaneous 3-pentanone PLIF images. Mean iso-contours of equivalence ratio (e.g.: $\phi=0.95$ and $\phi=1.05$ for near-stoichiometric flame regions) were then traced up to the mean position of the flame front at $\langle c\rangle=0.5$ for each gradient case, to define the width of the analysis ROI. This ensured fair comparison among gradient conditions, as the range of mean equivalence ratios was held constant by adjusting the width of the ROI based on the mean $\phi$ gradient. This approach was first used to study variations in local flame topology in the context of flame wrinkling scales, flame surface density, and curvature in near-stoichiometric flame regions. 
Results showed that stratification had a significant effect on flame wrinkling for globally stoichiometric flames, leading to enhanced corrugation of the flame front, and the formation of cusps with a relatively lower concentration of $\mathrm{OH}$. This was attributed to turbulent mixing, whereby locally rich reactant pockets $(\mathrm{Le}<1)$ were entrained by mixing of the imposed $\phi$ gradients at the exit of the burner. Somewhat surprisingly, variations in FSD were negligible among gradient conditions for nearstoichiometric flame regions, as variations in 2D flame length were offset by the increased turbulent flame brush thickness. PDFs of curvature also varied little with gradient.

The windowing approach was then used to quantify variations in HRR with gradient through the (OH LIF) $\left(\mathrm{CH}_{2} \mathrm{O}\right.$ LIF) approach developed by Paul et al. [55]. This marked the first implementation of this diagnostic to iso-octane/air flames, and numerical simulations were used to validate its use with isooctane/air mixtures. Potential temperature induced bias errors were estimated based on Boltzmann population fractions and estimated collisional quenching rates of both $\mathrm{OH}$ and $\mathrm{CH}_{2} \mathrm{O}$ species, and indicated that for the excitation transitions used, the product of raw (OH LIF) $\left(\mathrm{CH}_{2} \mathrm{O}\right.$ LIF) showed excellent correlation with the HRR over a broad range of premixed flame equivalence ratios. Conversely, the simulations also showed that while local, instantaneous measurements of HRR in a highly stratified flame would require temperature corrections, for an ROI with a symmetric $\phi$ distribution, potential bias errors on the ensemble averaged HRR could be accounted for given information on the range of equivalence ratios encountered. Thus, by matching the results of the simulations with the calculated range of equivalence ratios within each ROI (which varied among gradient settings due to turbulent mixing of the imposed mean $\phi$ gradients at the exit of the burner), bias errors could be determined via a Monte Carlo analysis. This extended analysis further ensured that potential variations in the mean HRR were directly attributable to the mean gradients in equivalence ratio.

Flame regions within a near-stoichiometric ROI showed a small but detectable decrease in HRR of up to $4.4 \%$ for the steepest gradient $\delta \phi / \delta y=0.029 \mathrm{~mm}^{-1}$ relative to the reference premixed case. This decrease in HRR was attributed to the decrease in thermal back-support from the heated products, as the temperature of adjacent products within the V-flame would be expected to decrease for reactants of weaker mixture strength. The general effect of stratification on the overall behaviour of a flame system was also 
separately evaluated for globally stoichiometric mixtures with different ranges in equivalence ratio. Results highlighted the presence of the competing effects of enhanced surface generation (up to 17.2\%) and reduced $\mathrm{HRR} /$ flame length (up to $22.5 \%$ ). Despite these competing effects, stratification lead to only a small reduction in total heat release rate of up to $9.1 \%$. These results further stressed the importance of clearly defining the question and measurement approach when attempting to discern specific effects of stratification on combustion. The influence of equivalence ratio gradient on local flame properties may differ significantly from the influence of general stratification on the global flame system in the form of different ranges of mixture strengths; distinguishing between these effects is critical to the proper interpretation of data, where both may be relevant in different applications.

The ROI approach was then extended to front- and back-supported rich, near-stoichiometric, and back-supported lean flame regions, as individual HRR images were evaluated for differences in peak HRR and instantaneous flame thickness $\delta_{\mathrm{t}}$ as the $\phi$ gradient was varied. The data were first studied for relative variations in local peak HRR and $\delta_{\mathrm{t}}$ with local curvature. A Monte Carlo error analysis was conducted to quantify both total and relative uncertainty of curvature measurements under different flame conditions, which to the author's knowledge is the first implementation of this approach to quantifying curvature uncertainties. Uncertainties in the relative local peak HRR and instantaneous flame thickness $\delta_{\mathrm{t}}$ were also estimated with the aid of a similar MC analysis.

Relative differences in local peak HRR and $\delta_{\mathrm{t}}$ were observed among the three major stoichiometries (premixed flames at $\bar{\phi}=0.9,1.0$, and 1.2). Trends were consistent with those expected from visual inspection of individual images, as the general distributions reflected the influence of competing effects of mass and thermal diffusion, consistent with thermodiffusive theory $[19,81]$. Subtle changes were observed among flame conditions for each of the three major stoichiometries, and while variations remained small, they did provide evidence of the potential interaction between neighbouring flame regions, suggesting that stratification may influence the local thermodiffusive stability of the wrinkled structures along a turbulent flame front.

Variations in the ensemble average of peak HRR and $\delta_{t}$ (i.e. the mean for all curvatures) were then compared among gradient settings, and differences relative to the corresponding reference-premixed case 
were examined. Local peak HRR and instantaneous flame thickness $\delta_{\mathrm{t}}$ showed opposite trends, with the increase in one leading to a decrease in the other. This was consistent for the three major stoichiometries studied. Small yet repeatable gradient effects were observed when comparing back- and front-supported locally rich flames, which experienced increases in peak HRR up to $10.2 \%\left(\delta \phi / \delta y=-0.014 \mathrm{~mm}^{-1}\right)$ and decreases up to $5.3 \%\left(\delta \phi / \delta y=0.012 \mathrm{~mm}^{-1}\right)$ respectively, coupled with a thinning of $\delta_{\mathrm{t}}$ of up to $7.2 \%$ and thickening of up to $2.4 \%$. Similar but weaker trends were observed for near-stoichiometric flame regions, with a decrease in peak HRR of up to $3.5 \%$ and a thickening of $\delta_{t}$ of up to $2.8 \%$ for the steepest gradient, $\delta \phi / \delta \mathrm{y}=0.029 \mathrm{~mm}^{-1}$. Locally lean, back-supported flame regions saw an increase in peak HRR up to $3.8 \%$, and corresponding decrease in $\delta_{\mathrm{t}}$ up to $2.1 \%$ for gradient case $\delta \phi / \delta \mathrm{y}=0.024 \mathrm{~mm}^{-1}$. While trends for backsupported lean flames were generally analogous to those of rich flames, differences were less pronounced for lean flames, further suggesting that the primary physical mechanisms of back-support (heat for $\phi<1$, species for $\phi>1$ ) may differ with local stoichiometry.

Overall, the data presented in this thesis indicate that mean $\phi$ gradients can influence the local dynamic behaviour of stratified, iso-octane/air turbulent V-flames. Though reported differences remained small among the relatively weak gradient flame settings considered here, it is important to note that these variations were observed within analysis ROI in which the mean range of $\phi$ remained constant, and were separate from the potential effects of local differences in mixture strength. Findings consistently provided evidence that $\phi$ gradients induced non-negligible interaction between neighbouring flame regions, and that these effects become more important as the gradient steepens. If these trends continue to the significantly steeper gradients that can be encountered in practical, partially premixed combustion systems, the current observations have important implications on future development. Design and optimization of exceedingly lean, severely stratified, next generation internal combustion devices thus hinges on the fundamental understanding of potential mixture gradient effects as a prerequisite to pollutant mitigation and environmental sustainability.

\subsection{Recommendations for Future Work}

While the measurements quantifying the effects of large-scale mean gradients in equivalence ratio on local flame front topology and HRR presented in the current work have provide new insights, they have 
also raised several questions that merit further investigation. The most important of these concerns further isolating the interaction between neighbouring flame regions in the context of identifying the physical and chemical phenomena that may alter combustion.

In the current experiments, turbulent flow properties were determined from LDV point measurements. Data were acquired along the exit plane of the burner, and limited to non-reacting conditions. The flow field could also be characterized with PIV under reacting conditions by seeding reactant air with solid $\mathrm{Al}_{2} \mathrm{O}_{3}$ particles to obtain velocity data through the flame, from reactants to products. The resulting measurements could then be used to determine the local flame propagation velocity, and compared to (OH LIF) $\left(\mathrm{CH}_{2} \mathrm{O}\right.$ LIF) HRR to study the correlation between both parameters among mean $\phi$ gradient conditions. Instantaneous $\mathrm{OH}$ and $\mathrm{CH}_{2} \mathrm{O}$ fluorescence signals must be corrected for temperature induced bias errors to obtain the local HRR along the stratified flame front, and information on the temperature field could be obtained from Rayleigh scattering [133] using a second Nd-YAG laser (frequency doubled to $532 \mathrm{~nm}$ ) and un-intensified CCD. Corrections for pulse-to-pulse variations in laser profile could also be obtained by reflecting a fraction of each laser sheet into a quartz cuvette filled with a fluorescing tracer [60,61], and imaging the respective LIF signals on each ICCD next to the $\mathrm{OH}$ and $\mathrm{CH}_{2} \mathrm{O}$ images. The same approach could be used to correct the instantaneous equivalence ratio images (from 3pentanone tracer PLIF), such that data could be further conditioned on the local, instantaneous mixture strength.

Additional experiments on stratified flames at higher turbulence levels relevant to practical combustion devices would be of interest. Though recently developed academic flame configurations such as stratified, axisymmetric swirl burners have begun to address this issue, there remains a significant lack of data available in the literature. These studies require the use of multicomponent diagnostics to effectively quantify the effects of stratification on the local reaction zone, and although 1D point measurement systems are currently viable and have been implemented [36], temporally resolved 2D and 3D data are required to fully characterize flame behaviour. Unfortunately, this implies that we remain restricted by the limitations, and financial feasibility of commercially available lasers and cameras. 
Laminar configurations capable of explicitly controlling the $\phi$ gradient, either normal or transverse to the flame front are also relevant to identifying the specific physical mechanisms that may influence the reaction zone. For instance, it would be interesting to study a planar flame in counterflow, where the $\phi$ gradient was transverse to the reaction zone. Counterflow flames provide a spatially stable, reproducible reaction zone that is ideal for the acquisition of statistically valid data. Several $\phi$ gradients could be studied at various local equivalence ratios, fuels, and strain rates. The 1D nature of these flames further lends itself to point measurements using currently available multicomponent diagnostic systems [36], and results could serve as validation data for DNS simulations. 


\section{References}

[1] World Energy Council, Transport Technologies and Policy Scenarios to 2050, 2007.

[2] J.W. Tester, E.M. Drake, M.J. Driscoll, M.W. Golay, W.A. Peters, Sustainable Energy, Choosing Among Options, Massachusetts Institute of Technology Press, 2005.

[3] N. Peters, Turbulent Combustion, Cambridge University Press, 2000.

[4] N. Pasquier, B. Lecordier, M. Trinité, A. Cessou, Proc. Combust. Inst. 31 (2007) 1567.

[5] B.P. Pundir, V.A. Zvonow, C.P. Gupta, SAE Tech. Ser. (1981) paper no. 810774.

[6] S. Kajitani, N. Sawa, K.T. Rhee, SAE Tech. Ser. (1990) paper no. 902142.

[7] Y. Miroyoshi, H. Morikawa, T. Kamimoto, T. Hayashi, SAE Tech. Ser. (1996) paper no. 962087.

[8] Y. Takagi, Proc. Combust. Inst. 27 (1998) 2055.

[9] B.D. Peters, A.A. Quader, SAE Tech. Ser. (1978) paper no. 780234.

[10] C. Poppe, S. Sivasegaram, J.H. Whitelaw, Control of NOx Emissions in Confined Flames by Oscillations, Report No. TF/96/09, Imperial College, London, England, 1996.

[11] F. Zhao, M.-C. Lai, D.L. Harrington, Prog. Energy Combust. Sci. 25 (1999) 437.

[12] S. Shiga, S. Ozone, H.T.C. Machacon, T. Karasawa, H. Nakamura, T. Ueda, N. Jingu, Z. Huang, M. Tsue, M. Kono, Combust. Flame 129 (2002) 1.

[13] T. Date, S. Yagi, A. Ishizuka, I. Fujii, SAE Tech. Ser. (1974) paper no. 740605.

[14] T. Inoue, S. Matsushita, K. Nakanishi, H. Okano, SAE Tech. Ser. (1993) paper no. 930873.

[15] H. Ando, in:, Int. Mech. Eng. Semin. Lean Burn Combust. Engines, 1996, p. S433.

[16] Y.-S. Cho, D.A. Santavicca, SAE Tech. Ser. (1993) paper no. 932715.

[17] J. Zhou, K. Nishida, T. Yoshizaki, H. Hiroyasu, SAE Tech. Ser. (1998) paper no. 982563.

[18] N. Peters, Proc. Combust. Inst. 21 (1988) 1231.

[19] F.A. Williams, Combustion Theory: The Fundamental Theory of Chemically Reacting Flow Systems, 2nd ed., The Benjamin/Cummings Publishing Company Inc., 1985. 
[20] F.E. Marble, J.E. Broadwell, The Coherent Flame Model for Turbulent Chemical Reactions Project Squid Technical Report (TRW-9-PU), Purdue University, West Lafayette, IN, 1977.

[21] Y. Ra, W.K. Cheng, in:, Fifth Int. Symp. Diagnostics Model. Combust. Intern. Combust. Engines, 2001, pp. 251-257.

[22] T. Kang, D. Kyritsis, Combust. Sci. Tech. 177 (2005) 2191.

[23] T. Kang, D.C. Kyritsis, Proc. Combust. Inst. 31 (2007) 1075.

[24] T. Kang, D. Kyritsis, Energy Convers. Manag. 48 (2007) 2769.

[25] T. Kang, D. Kyritsis, Proc. Combust. Inst. 32 (2009) 979.

[26] A. Pires Da Cruz, A.M. Dean, J.M. Grenda, Proc. Combust. Inst. 28 (2000) 1925.

[27] C.M. Lund, HCT: A General Computer Program for Calculating Time-Dependent Phenomena Involving One-Dimensional Hydrodynamics, Transport and Detailed Chemical Kinetics, Technical Report UCRL-52504, Lawrence Livermore National Laboratory, 1978.

[28] F.N. Egolfopoulos, P. Cho, C.K. Law, Combust. Flame 76 (1989) 375.

[29] V. Robin, A. Mura, M. Champion, O. Degardin, B. Renou, M. Boukhalfa, Combust. Flame 153 (2008) 288.

[30] O. Dégardin, Effets Des Hétérogénéités de Richesse Sur La Structure Locale Des Flammes Turbulentes, Ph.D., Institut National des Sciences Appliquees de Rouen, France, 2006.

[31] M.S. Sweeney, S. Hochgreb, R.S. Barlow, Combust. Flame 158 (2011) 935.

[32] M.S. Sweeney, S. Hochgreb, M.J. Dunn, R.S. Barlow, Combust. Flame 159 (2012) 2896.

[33] M.S. Sweeney, S. Hochgreb, M.J. Dunn, R.S. Barlow, Combust. Flame 159 (2012) 2912.

[34] M.S. Sweeney, S. Hochgreb, M.J. Dunn, R.S. Barlow, Combust. Flame 160 (2013) 322.

[35] A. Bonaldo, J.B. Kelman, Combust. Flame 156 (2009) 750.

[36] R.S. Barlow, G.-H. Wang, P. Anselmo-Filho, M.S. Sweeney, S. Hochgreb, Proc. Combust. Inst. 32 (2009) 945.

[37] A. Benarous, D. Karmed, A. Liazid, J. Mech. Sci. Technol. 27 (2013) 2557.

[38] O. Dégardin, B. Renou, A.M. Boukhalfa, Exp. Fluids 40 (2006) 452.

[39] D. Garrido-López, S. Sarkar, Proc. Combust. Inst. 30 (2005) 621.

[40] C. Pera, S. Chevillard, J. Reveillon, Combust. Flame 160 (2013) 1020.

[41] B. Renou, E. Samson, A. Boukhalfa, Combust. Sci. Tech. 176 (2004) 1867. 
[42] C. Galizzi, D. Escudié, Combust. Flame 145 (2006) 621.

[43] C. Galizzi, D. Escudié, Combust. Flame 157 (2010) 2277.

[44] P.C. Vena, B. Deschamps, G.J. Smallwood, M.R. Johnson, in:, SAE 2009 Powertrains Fuels Lubr. Meet., SAE Technical Paper 2009-01-2655, San Antonio, TX, 2009, pp. 1-9.

[45] P. Anselmo-Filho, S. Hochgreb, R.S. Barlow, R.S. Cant, Proc. Combust. Inst. 32 (2009) 1763.

[46] B. Böhm, J.H. Frank, A. Dreizler, Proc. Combust. Inst. 33 (2011) 1583.

[47] P.C. Vena, B. Deschamps, G.J. Smallwood, M.R. Johnson, Proc. Combust. Inst. 33 (2011) 1551.

[48] M.S. Sweeney, S. Hochgreb, M.J. Dunn, R.S. Barlow, Proc. Combust. Inst. 33 (2011) 1419.

[49] E. Samson, Etude Experimentale de La Propagation de Flammes En Expansion Dans Un Milieu a Richesse Stratifiee, Ph.D., L'Institut National des Sciences Appliquees de Rouen, 2002.

[50] C. Galizzi, Étude Expérimentale Du Développement D’une Flame Oblique Prémélangée Dans Un Écoulement Stratifié, École Centrale de Lyon, 2003.

[51] F. Seffrin, F. Fuest, D. Geyer, a. Dreizler, Combust. Flame 157 (2010) 384.

[52] G. Kuenne, F. Seffrin, F. Fuest, T. Stahler, A. Ketelheun, D. Geyer, J. Janicka, A. Dreizler, Combust. Flame 159 (2012) 2669.

[53] V. Robin, A. Mura, M. Champion, P. Plion, Combust. Sci. Technol. 178 (2006) 1843.

[54] P.A. Libby, F.A. Williams, Combust. Sci. Technol. 161 (2000) 351.

[55] P.H. Paul, H.N. Najm, Proc. Combust. Inst. 27 (1998) 43.

[56] A. Fayoux, K. Zähringer, O. Gicquel, J.C. Rolon, Proc. Combust. Inst. 30 (2005) 251.

[57] B.O. Ayoola, R. Balachandran, J.H. Frank, E. Mastorakos, C.F. Kaminski, Combust. Flame 144 (2006) 1.

[58] R. Balachandran, B. Ayoola, C. Kaminski, A. Dowling, E. Mastorakos, Combust. Flame 143 (2005) 37.

[59] S. Böckle, J. Kazenwadel, T. Kunzelmannn, D.-I. Shin, C. Schulz, J. Wolfrum, Proc. Combust. Inst. $28(2000) 279$.

[60] M. Röder, T. Dreier, C. Schulz, Appl. Phys. B 107 (2012) 611.

[61] M. Röder, T. Dreier, C. Schulz, Proc. Combust. Inst. 34 (2013) 3549.

[62] Y. Hardalupas, C.S. Panoutsos, A.M.K.P. Taylor, Exp. Fluids 49 (2010) 883.

[63] C.M. Vagelopoulos, J.H. Frank, Proc. Combust. Inst. 30 (2005) 241. 
[64] R.L. Gordon, A.R. Masri, E. Mastorakos, Combust. Flame 155 (2008) 181.

[65] R.L. Gordon, A.R. Masri, E. Mastorakos, Combust. Theory Model. 13 (2009) 645.

[66] T. Poinsot, D. Veynante, A. Trouve, G. Ruetsch, in:, Proc. CTR Summer Progr., Center for Turbulence Research, NASA Ames/Stanford University, USA, 1996, pp. 111-136.

[67] D.C. Haworth, R.J. Blint, B. Cuenot, T.J. Poinsot, Combust. Flame 121 (2000) 395.

[68] C. Jiménez, B. Cuenot, T. Poinsot, D. Haworth, Combust. Flame 128 (2002) 1.

[69] J. Helie, A. Trouve, P. Combust. Inst. 27 (1998) 891.

[70] Y.M. Marzouk, A.F. Ghoniem, H.N. Najm, Proc. Combust. Inst. 28 (2000) 1859.

[71] Y. Ra, W.K. Cheng, in:, Fifth Int. Symp. Diagnostics Model. Combust. Intern. Combust. Engines, 2001, pp. 251-257.

[72] E.S. Richardson, V.E. Granet, A. Eyssartier, J.H. Chen, Combust. Theory Model. 14 (2010) 775.

[73] S.K. Lele, J. Comput. Phys. 103 (1992) 16.

[74] T.J. Poinsot, S.K. Lele, J. Comput. Phys. 101 (1992) 104.

[75] J. Warnatz, Proc. Combust. Inst. 18 (1981) 369.

[76] M.D. Smooke, I.K. Puri, K. Seshadri, Proc. Combust. Inst. 21 (1986) 1783.

[77] A.E. Lutz, R.J. Kee, J.F. Grear, F.M. Rupley, OPPDIF: A FORTRAN Program for Com- Puting Opposed-Flow Diffusion Flames, Technical Report No. SAND96-8243, Sandia National Laboratories, 1996.

[78] H.G. Im, L.L. Raja, R.J. Kee, L.R. Petzold, A FORTRAN Program for Unsteady Opposed-Flow Flames, Technical Report No. SAND2000-8211, Sandia National Laboratories, 2000.

[79] R.J. Kee, F.M. Rupley, J.A. Miller, Chemkin-II: A FORTRAN Chemical Kinetics Package for the Analysis of Gas-Phase Chemical Kinetics, Technical Report No. SAND89-8009B, Sandia National Laboratories, 1989.

[80] R. Zhou, S. Hochgreb, Combust. Flame 160 (2013) 1070.

[81] N. Chakraborty, R.S. Cant, Phys. Fluids 17 (2005) 105105.

[82] Y.-C. Chen, M. Kim, J. Han, S. Yun, Y. Yoon, Proc. Combust. Inst. 31 (2007) 1327.

[83] S. Einecke, C. Schulz, V. Sick, Appl. Phys. B Lasers Opt. 71 (2000) 717.

[84] H. Neij, B. Johansson, M. Alden, Combust. Flame 99 (1994) 449.

[85] B.M. Deschamps, G.J. Smallwood, J. Prieur, D.R. Snelling, O.L. Gulder, Proc. Combust. Inst. 26 (1996) 427. 
[86] S.B. Pope, Int. J. Eng. Sci. 26 (1988) 445.

[87] S. Pfadler, F. Beyrau, M. Löffler, A. Leipertz, Opt. Express 14 (2006) 10171.

[88] I.G. Shepherd, Proc. Combust. Inst. 26 (1996) 373.

[89] F.C. Gouldin, P.C. Miles, Combust. Flame 100 (1995) 202.

[90] T. Lee, G.L. North, D.A. Santavicca, Combust. Flame 93 (1993) 445.

[91] X.J. Gu, M.Z. Haq, M. Lawes, R. Woolley, Combust. Flame 121 (2000) 41.

[92] A.C. Alkidas, Energy Convers. Manag. 48 (2007) 2751.

[93] J.E. Harrington, K.C. Smyth, Chem. Phys. Lett. 202 (1993) 196.

[94] D.C. Kyritsis, V.S. Santoro, A. Gomez, Exp. Fluids 37 (2004) 769.

[95] S.G. Davis, C.K. Law, Combust. Flame 27 (1998) 521.

[96] X. Zhang, C. Tang, H. Yu, Q. Li, J. Gong, Z. Huang, Energy Fuel 27 (2013) 2327.

[97] J.E. Freeh, K. Kumar, Y. Huang, C.J. Sung, in:, 40th AIAA/ASME/SAE/ASEE Jt. Propuls. Conf. Exhib., 2004, p. 10.

[98] H. Takashi, T. Kimitoshi, in:, FISITA, 2006.

[99] A.P. Kelley, W. Liu, Y.X. Xin, A.J. Smallbone, C.K. Law, Proc. Combust. Inst. 33 (2011) 501.

[100] M. Sweeney, S. Hochgreb, Appl. Opt. 48 (2009) 3866.

[101] C. Schulz, V. Sick, Prog. Energy Combust. Sci. 31 (2005) 75.

[102] J.D. Koch, R.K. Hanson, Appl. Phys. B Lasers Opt. 76 (2003) 319.

[103] F. Ossler, M. Aldén, Appl. Phys. B Lasers Opt. 64 (1997) 493.

[104] J.D. Koch, J. Gronki, R.K. Hanson, J. Quant. Spectrosc. Radiat. Transf. 109 (2008) 2037.

[105] J. Nygren, J. Hult, M. Richter, M. Alden, M. Christensen, A. Hultqvist, B. Johnansson, Proc. Combust. Inst. 29 (2002) 679.

[106] R.C. Gonzalez, R.E. Woods, S.L. Eddins, Digital Image Processing Using MATLAB, Prentice Hall, 2004.

[107] J.R. Janesick, Scientific Charge-Coupled Devices, SPIE Optical Engineering Press, Bellingham, 2001.

[108] H.N. Najm, O.M. Knio, P.H. Paul, P.S. Wyckoff, Combust. Sci. Tech. 140 (1998) 369. 
[109] H.N. Najm, P.H. Paul, C.J. Mueller, P.S. Wyckoff, Combust. Flame 113 (1998) 312.

[110] R.J. Kee, J.F. Grcar, M.D. Smooke, J.A. Miller, A Fortran Program for Modelling Steady Laminar One-Dimensional Premixed Flames, Report No. SAND 85-8240, Sandia National Laboratories, 1985.

[111] S. Jerzembeck, N. Peters, P. Pepiotdesjardins, H. Pitsch, Combust. Flame 156 (2009) 292.

[112] R.J. Kee, J.A. Miller, T.H. Jefferson, A General-Purpose, Problem-Independent, Transportable, Fortran Chemical Kinetics Code Package, Report No. SAND 80-8003, Sandia National Laboratories, 1980.

[113] R.J. Kee, J. Warnatz, J.A. Miller, A Fortran Computer Code Package for the Evaluation of GasPhase Viscosities, Conductivities, and Diffusion Coefficients, Report No. SAND 83-8209, Sandia National Laboratories, 1983.

[114] B. Ayoola, G. Hartung, C.A. Armitage, J. Hult, R.S. Cant, C.F. Kaminski, Exp. Fluids 46 (2008) 27.

[115] C. Brackmann, J. Nygren, X. Bai, Z. Li, H. Bladh, B. Axelsson, I. Denbratt, L. Koopmans, P.-E. Bengtsson, M. Aldén, Spectrochim. Acta Part A 59 (2003) 3347.

[116] K. Yamamoto, S. Isii, M. Ohnishi, Proc. Combust. Inst. 33 (2011) 1285.

[117] J. Luque, D.R. Crosley, LIFBASE: Database and Spectral Simulation Program, SRI International Report MP 99-009, 1999.

[118] M. Tamura, P.A. Berg, J.E. Harrington, J. Luque, J.B. Jeffries, G.P. Smith, D.R. Crosley, Combust. Flame 114 (1998) 502.

[119] D.J. Clouthier, D.A. Ramsay, Annu. Rev. Phys. Chem. 34 (1983) 31.

[120] The San Diego Mechanism, Center for Energy Research, University of California at San Diego, 2003.

[121] Y. Nakamura, S. Manome, H. Yamashita, in:, 13th Int. Symp. Appliications Laser Tech. to Fluid Mech., Lisbon, Portugal, 2006, pp. 1-10.

[122] M. Richter, R. Collin, J. Nygren, M. Alden, L. Hildingsson, B. Johansson, JSME Int. Journal, Ser. B 48 (2005) 701.

[123] D. Bradley, C.G.W. Sheppard, R. Woolley, D.A. Greenhalgh, R.D. Lockett, Combust. Flame 122 (2000) 195.

[124] R. Lockett, R. Woolley, Combust. Flame 151 (2007) 601.

[125] A.S. Al-Shahrany, D. Bradley, M. Lawes, K. Liu, R. Woolley, Combust. Sci. Technol. 178 (2006) 1771.

[126] J.K. Bechtold, M. Matalon, Combust. Flame 67 (1987) 77.

[127] D. Bradley, Philos. Trans. R. Soc. A 357 (1999) 3567. 
[128] P.C. Vena, B. Deschamps, H. Guo, G.J. Smallwood, M.R. Johnson, Submitt. to Combust. Flame (2014).

[129] N.T. Clemens, in:, J.P. Hornak (Ed.), Encycl. Imaging Sci. Technol., John Wiley \& Sons, Ltd, 2002, pp. 390-419.

[130] L.P.H. de Goey, T. Plessing, R.T.E. Hermanns, N. Peters, Proc. Combust. Inst. 30 (2005) 859.

[131] M.Z. Haq, C.G.W. Sheppard, R. Woolley, D.A. Greenhalgh, R.D. Lockett, Combust. Flame 131 (2002) 1.

[132] R.S.M. Chrystie, I.S. Burns, J. Hult, C.F. Kaminski, Meas. Sci. Technol. 19 (2008) 125503.

[133] J.H. Frank, S.A. Kaiser, M.B. Long, Proc. Combust. Inst. 29 (2002) 2687. 


\section{Appendix A Determination of the Effective Spatial Resolution of the Optical Imaging System}

The effective spatial resolution of an optical system is a measure of its ability to accurately image contrast variations in the object plane, i.e the degree of blurring in an image. Effective spatial resolution is generally quantified from the optical transfer function (OTF), which can be approximated from the Fourier transform of the line spread function (LSF). In the current study, the LSF was calculated from the derivative of the step response function (SRF), which was experimentally determined using the scanning knife-edge method [129]. Figure A.1 shows the apparatus used for this for this measurement, which includes a Princeton instrument ICCD equipped with a Nikkor $105 \mathrm{~mm}$ lens, a Wilkinson Sword classic razor blade mounted on a Newport 426 linear translation stage with a SM-25 vernier micrometer, and a SphereOptics LLC 4" integrating sphere. The optical system was setup to match the configuration used during flame experiments with an $\mathrm{f} \#=4.5$, and projected spatial resolution of $67 \mu \mathrm{m} / \mathrm{pixel}$.

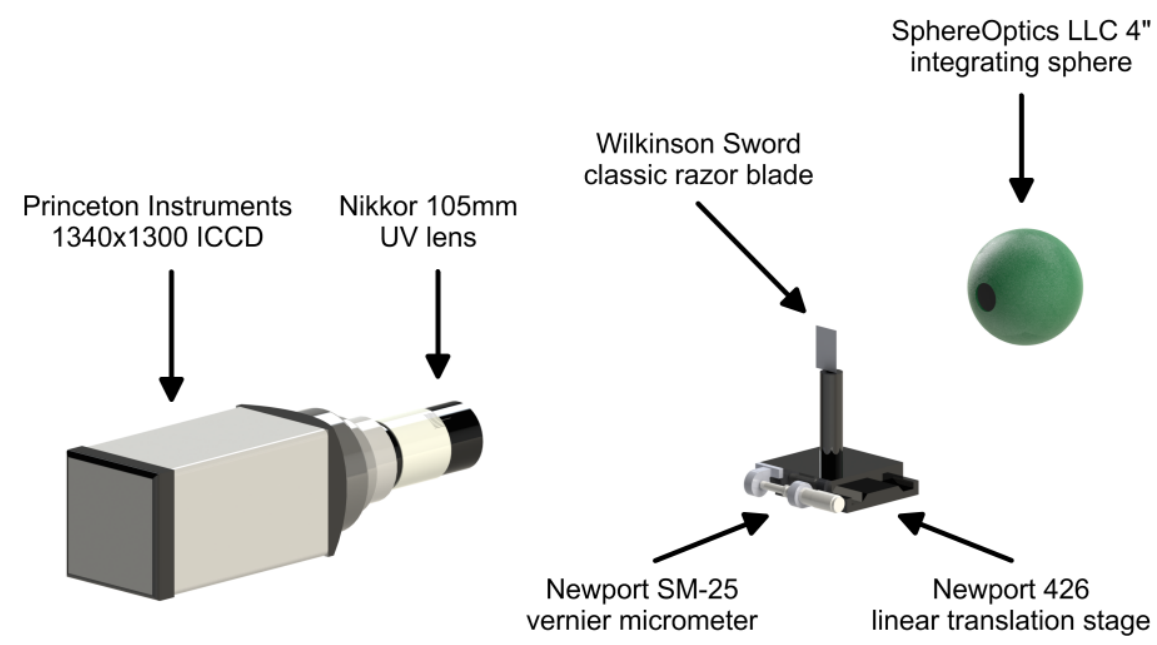

Figure A.1 Experimental setup for the determination of the step response function (SRF) of a single pixel on an ICCD using the scanning knife-edge technique. 
The SRF was obtained by recording the intensity of a single pixel in the middle of the CCD as the knife-edge was translated across the object plane at $20 \mu \mathrm{m}$ intervals. Twenty instantaneous images were acquired at each position of the translation, and the corresponding mean pixel count of a single pixel is plotted in Figure A.2 as a function knife-edge position. An error function was then fit to the data before the Gaussian LSF was determined by differentiating the fit. Results show a $1 / \mathrm{e}^{2}$ full width at half maximum of roughly $263 \mu \mathrm{m}$, which equates to an effective spatial resolution of 3.91 pixels.

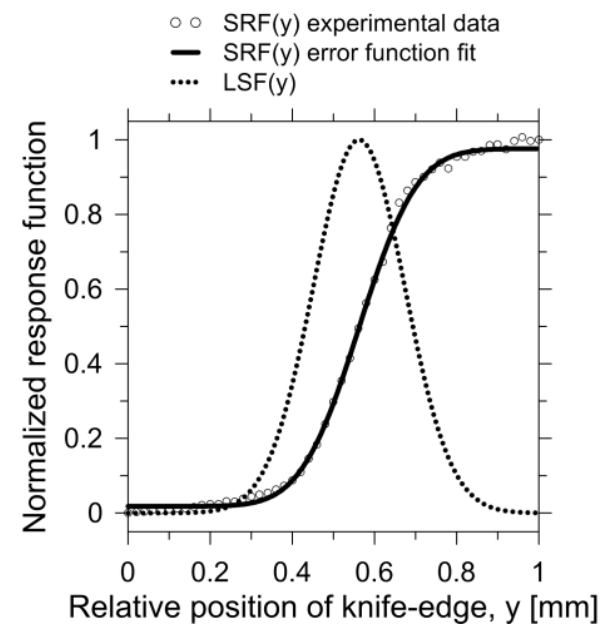

Figure A.2 Experimentally measured SRF, corresponding error function fit, and Gaussian LSF. 


\section{Appendix B Supplemental Photographs, Manufacturing Drawings, and Schematics of the Experimental Apparatus, NRC Stratified Slot Burner, and Iso-Octane/Air Delivery System}

This appendix provides further details on the layout of the experiments, the design of the NRC stratified

slot burner, and the iso-octane/air delivery system. Additional photographs are provided in Section B.1 to help the reader picture the overall set-up and key components of the apparatus. Fully dimensioned manufacturing drawings of the NRC stratified slot burner are included in Section B.2. A general schematic outlining each component of the flow control system used to deliver iso-octane/air mixtures to the burner is shown in Section B.3. 


\section{B.1 Detailed Photographs of the Experimental Apparatus}

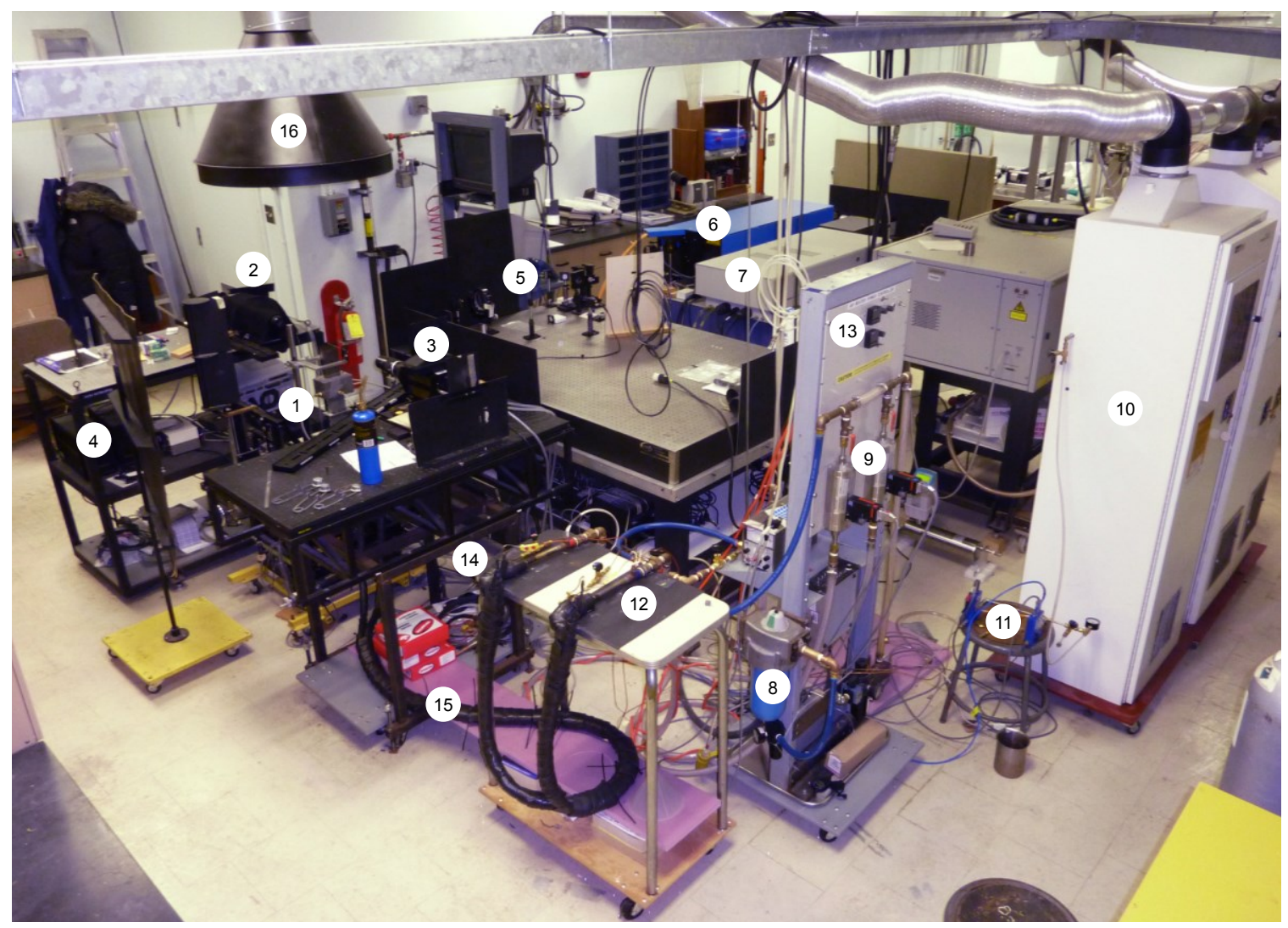

Figure B.1 General overview of the experimental apparatus.

(1) NRC stratified slot burner and 3-axis traverse system

(2) Princeton Instrument PIMAX $1340 \times 1300$ ICCD used for OH and 3-pentanone PLIF

(3) Princeton Instrument PIMAX $1340 \times 1300$ ICCD used for $\mathrm{CH}_{2} \mathrm{O}$ and biacetyl PLIF

(4) ST-133 controllers for ICCDs

(5) sheet forming optics

(6) Sirah Precision Scan Rhodamine B Dye laser

(7) dual head Quanta Ray PIV400 YAG laser

(8) Sperry Vickers particulate air filter

(9) Brooks Smart Series gas MFCs (5853S/BE 200 SLPM air full scale)

(10) storage cabinet containing iso-octane fuel tank and $\mathrm{N}_{2}$ bottle (pressurized $\mathrm{N}_{2}$ was used to push the liquid iso-octane through the MFCs)

(11) Horiba LV-F liquid MFCs (LF-F60MO $40 \mathrm{ccm}$ iso-octane full scale, LF-F50MO-094 $25 \mathrm{ccm}$ isooctane full scale)

(12) Omegalux AHP-7561 In-Line Air Heaters

(13) Omega display panel meter

(14) location of iso-octane injection to heated air-lines

(15) premixed iso-octane/air reactant lines

(16) exhaust hood 

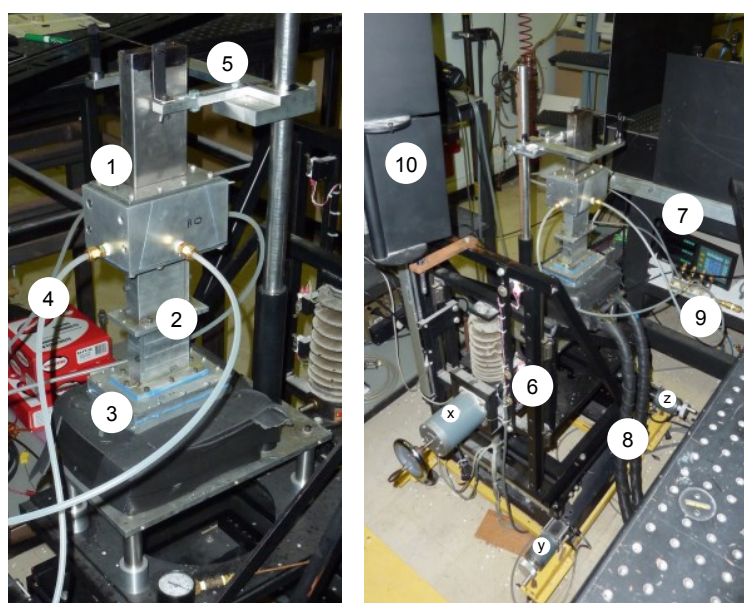

Figure B.2 Detailed view of NRC stratified burner and 3-axis traverse system.

(1) transverse mixing section and air co-flow

(2) lateral mixing sections (a) and (b)

(3) lower mixing section

(4) co-flow inlet air-lines

(5) flame stabilizing bar and displacement mechanism

(6) 3-axis traverse consisting of an in-house built base frame, Daedall 318122S-10E-LH linear table, Daedall micro-controlled stepper motors, and Mitutoyo AT11-N300 optical linear encoders

(7) Mitutoyo linear scale digital readout PL-335L

(8) fully-premixed iso-octane/air inlet lines to lower mixing section of burner

(9) air co-flow distribution manifold to 4 inlet lines

(10) beam dump

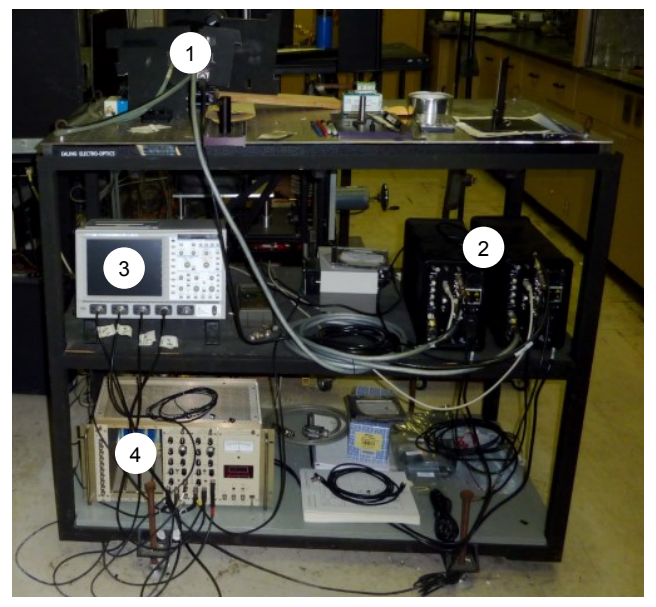

Figure B.3 Detailed view of ICCD controllers.

(1) Princeton Instrument PIMAX 1340 x 1300 ICCD

(2) Princeton Instrument ST-133 controllers

(3) 4 channel LeCroy LC334A $500 \mathrm{Mhz}$ oscilloscope

(4) Stanford Research Systems SR280 Boxcar Averager System mainframe outfitted with two SR250 Boxcar Gated Integrator Modules (one for each laser beam) 


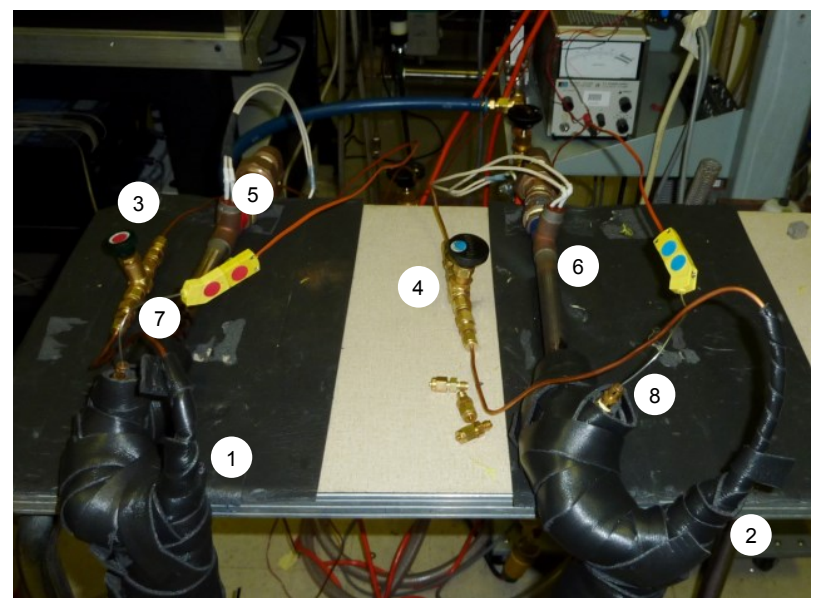

Figure B.4 Detailed view of iso-octane inlets to heated air-lines.

(1) and (2) location of iso-octane inlets to air-lines

(3) and (4) iso-octane liquid fuel lines

(5) and (6) Omegalux AHP-7561 in-line air heaters

(7) and (8) K-type thermocouples used to monitor air temperature at exit of heaters

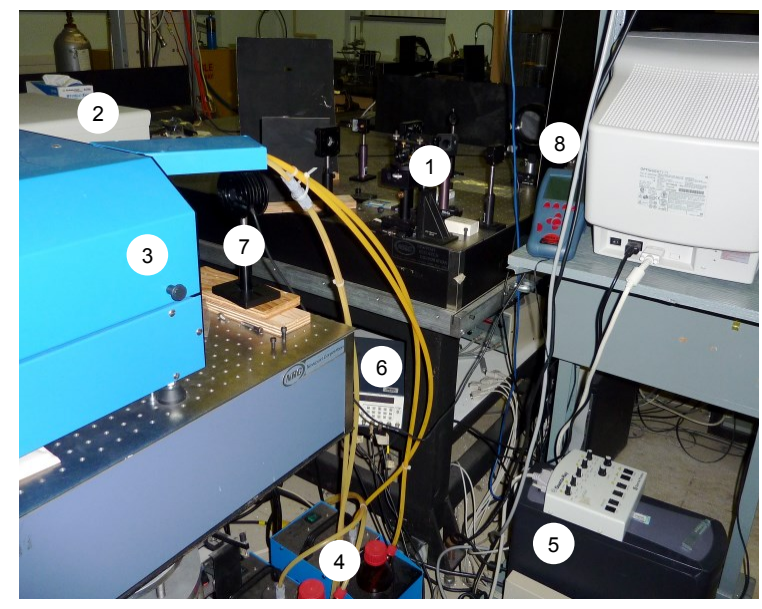

Figure B.5 Detailed view of set-up of lasers and optics.

(1) sheet forming optics

(2) dual head Quanta Ray PIV400 YAG laser

(3) Sirah Precision Scan Rhodamine B Dye laser

(4) dye circulator system equipped with two dye cells: resonator / pre-amplifier cell and capillary cell

(5) Quanta-Ray PIV-400 controller

(6) DG-535 Stanford pulse generator

(7) Ophir laser thermal sensor 30 (150) A-HE

(8) Ophir NOVA II display 


\section{B.2 Manufacturing Drawings of the NRC Stratified Slot Burner}

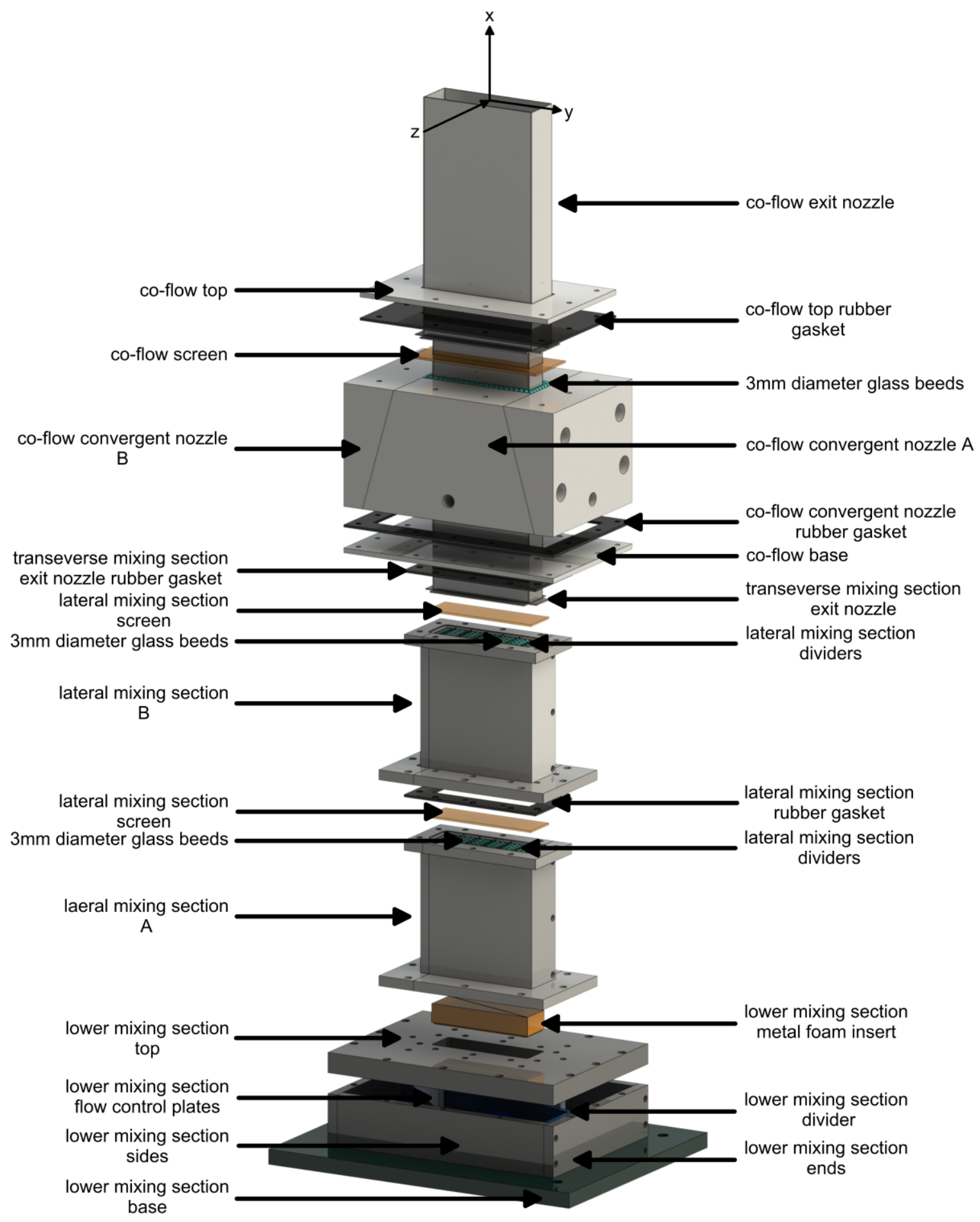

Figure B.6 Exploded view of NRC stratified slot burner (the $1.5 \mathrm{~mm}$ diameter stabilization rod that was aligned in z-direction and positioned at $x=5 \mathrm{~mm}$ and $y=16 \mathrm{~mm}$ is not shown in the figure). 
NRC stratified burner

lower mixing section base
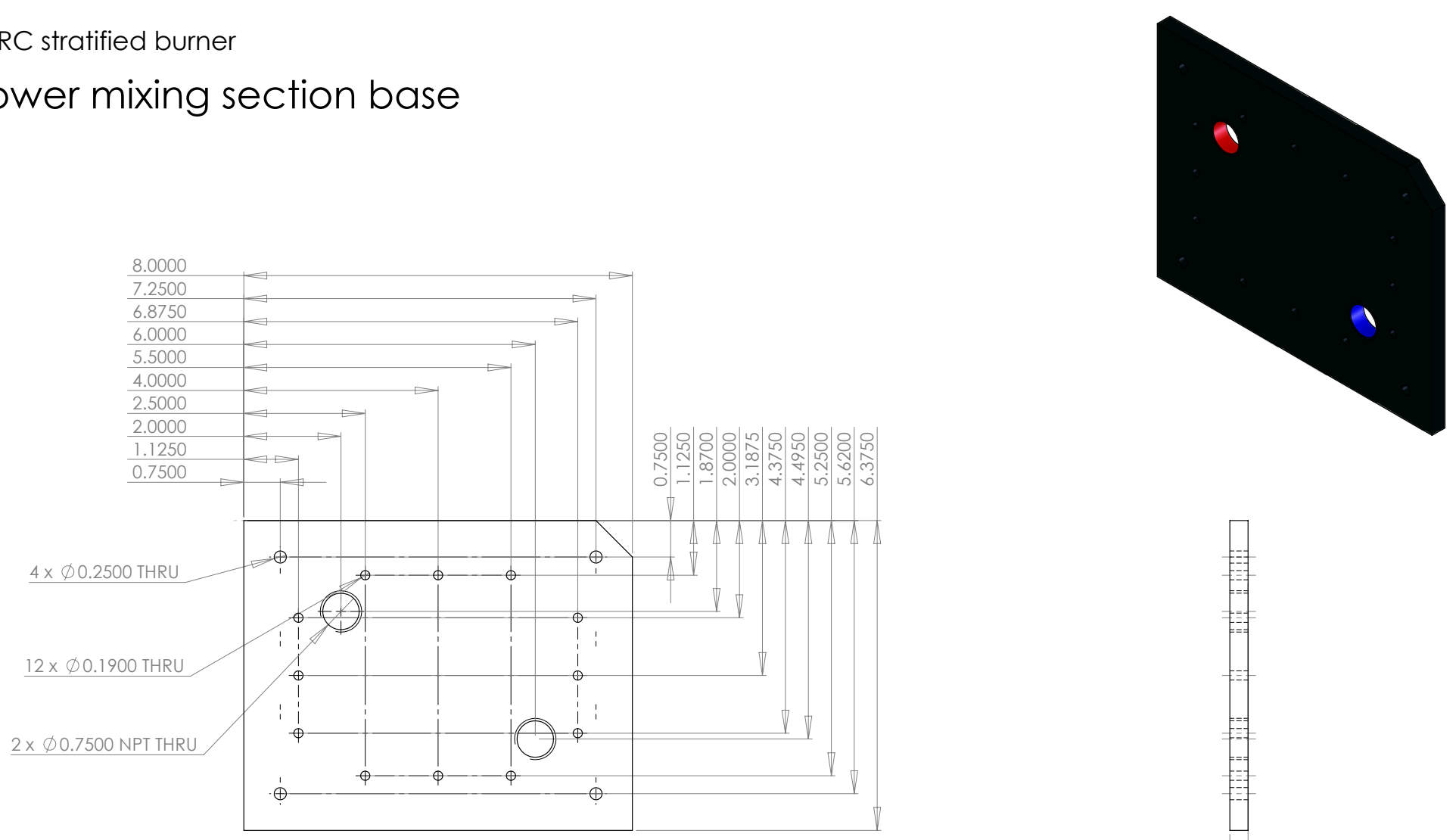

MATERIAL : aluminum

ALL DIMENSIONS +/-0.005

1 OFF 
NRC stratified burner

lower mixing section sides
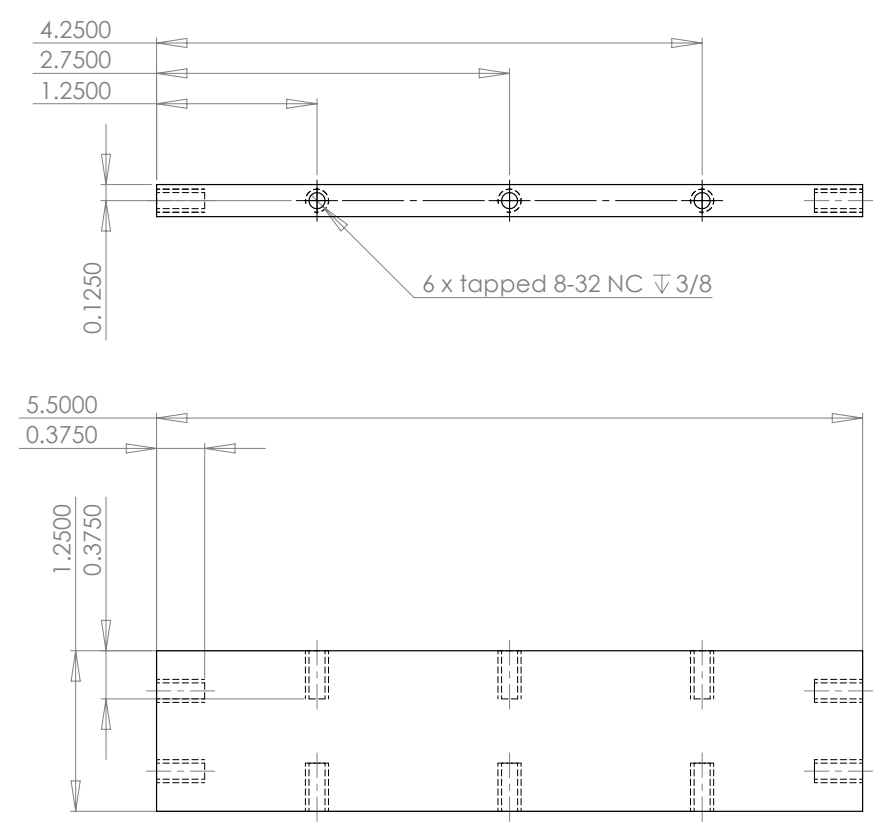
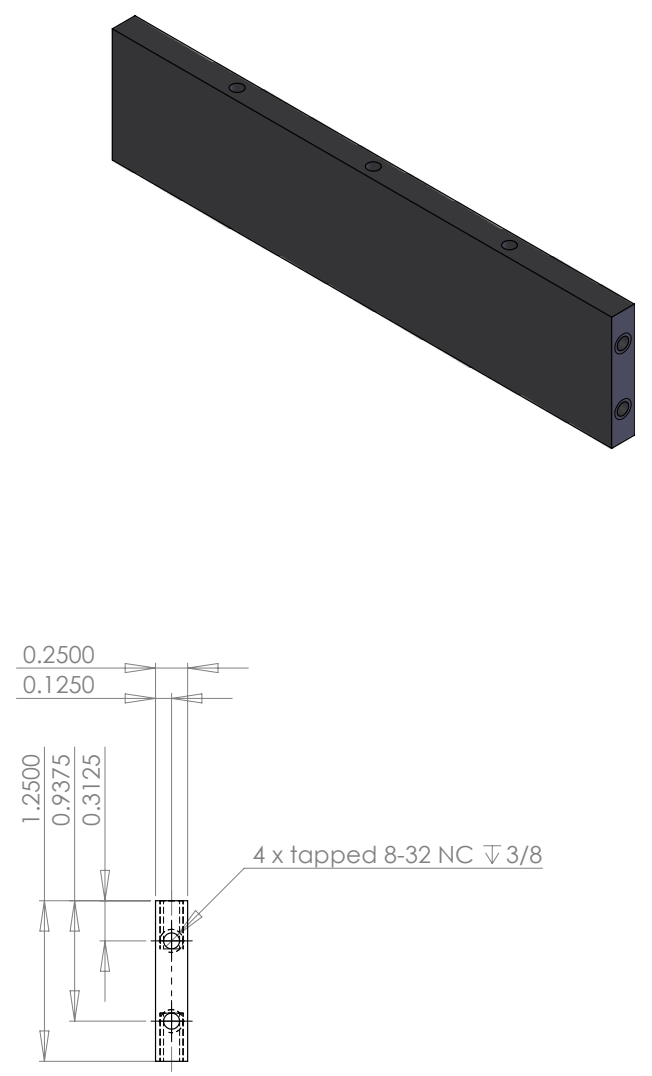

MATERIAL : aluminum

ALL DIMENSIONS +/-0.005

2 OFF 
NRC stratified burner

lower mixing section ends
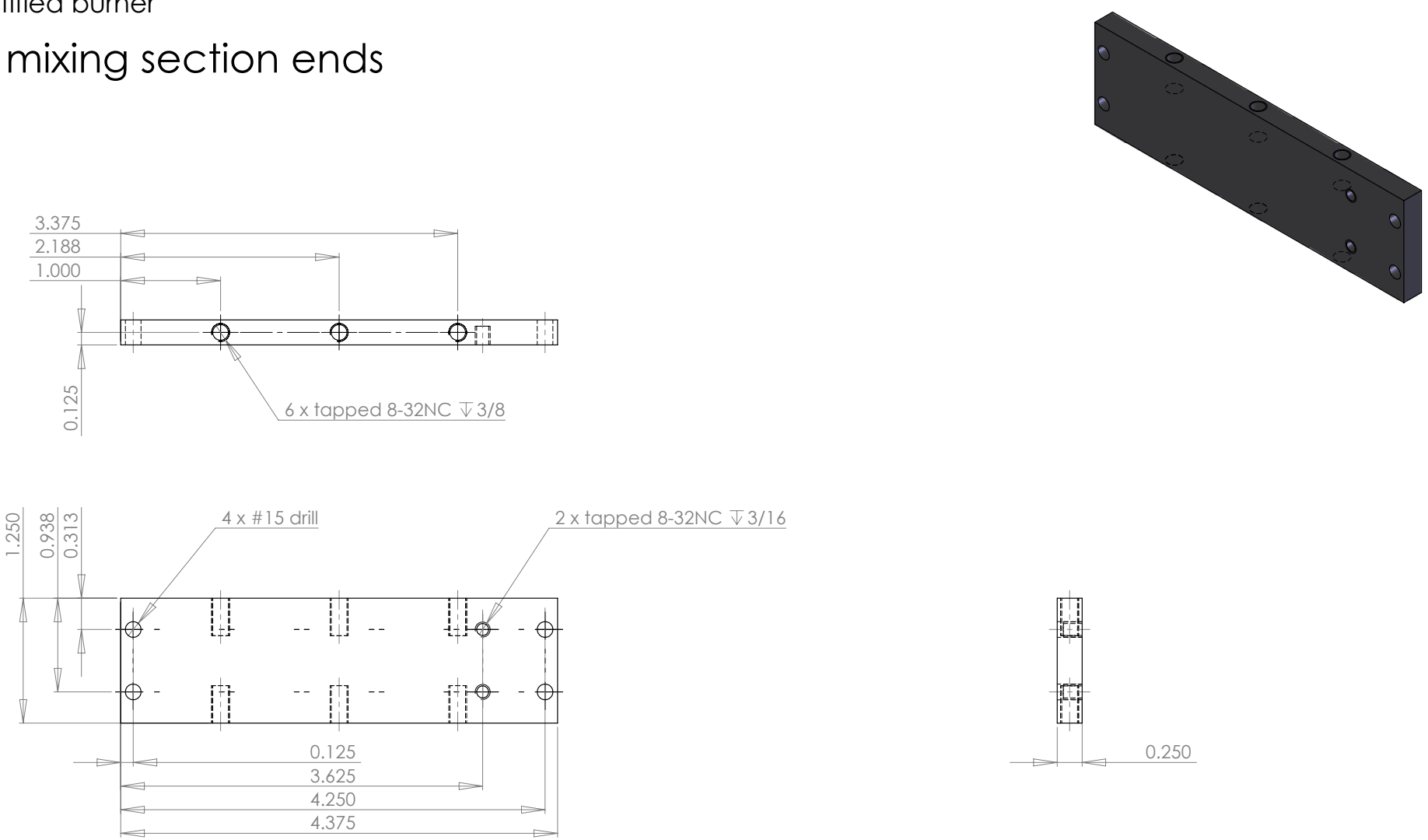

MATERIAL : aluminum

ALL DIMENSIONS +/-0.005

2 OFF 
NRC stratified burner

lower mixing section divider
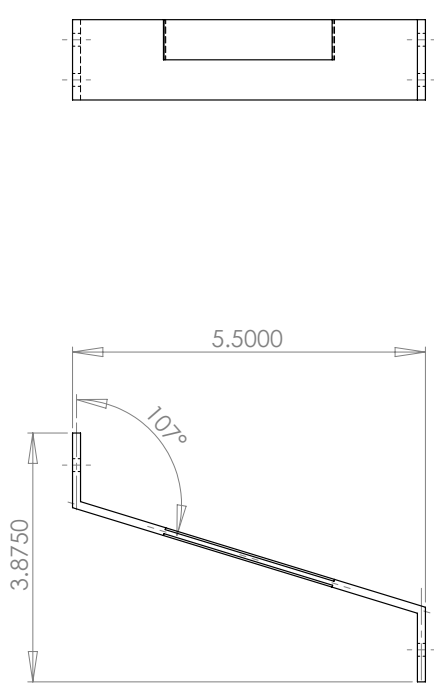
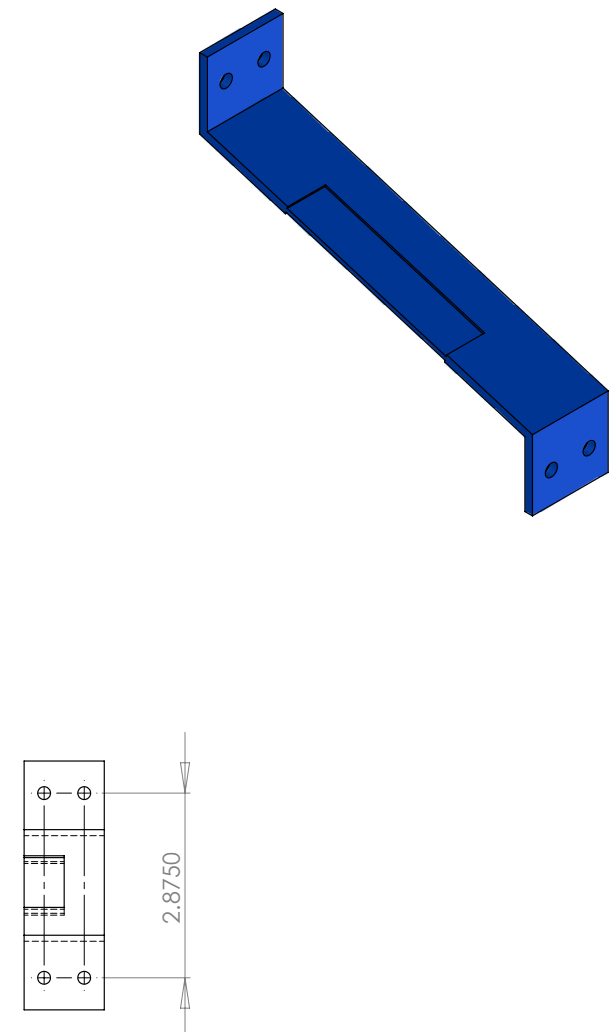

MATERIAL : aluminum

ALL DIMENSIONS +/-0.005

$1 \mathrm{OFF}$ 
NRC stratified burner

lower mixing section divider

dimensions of flat stock
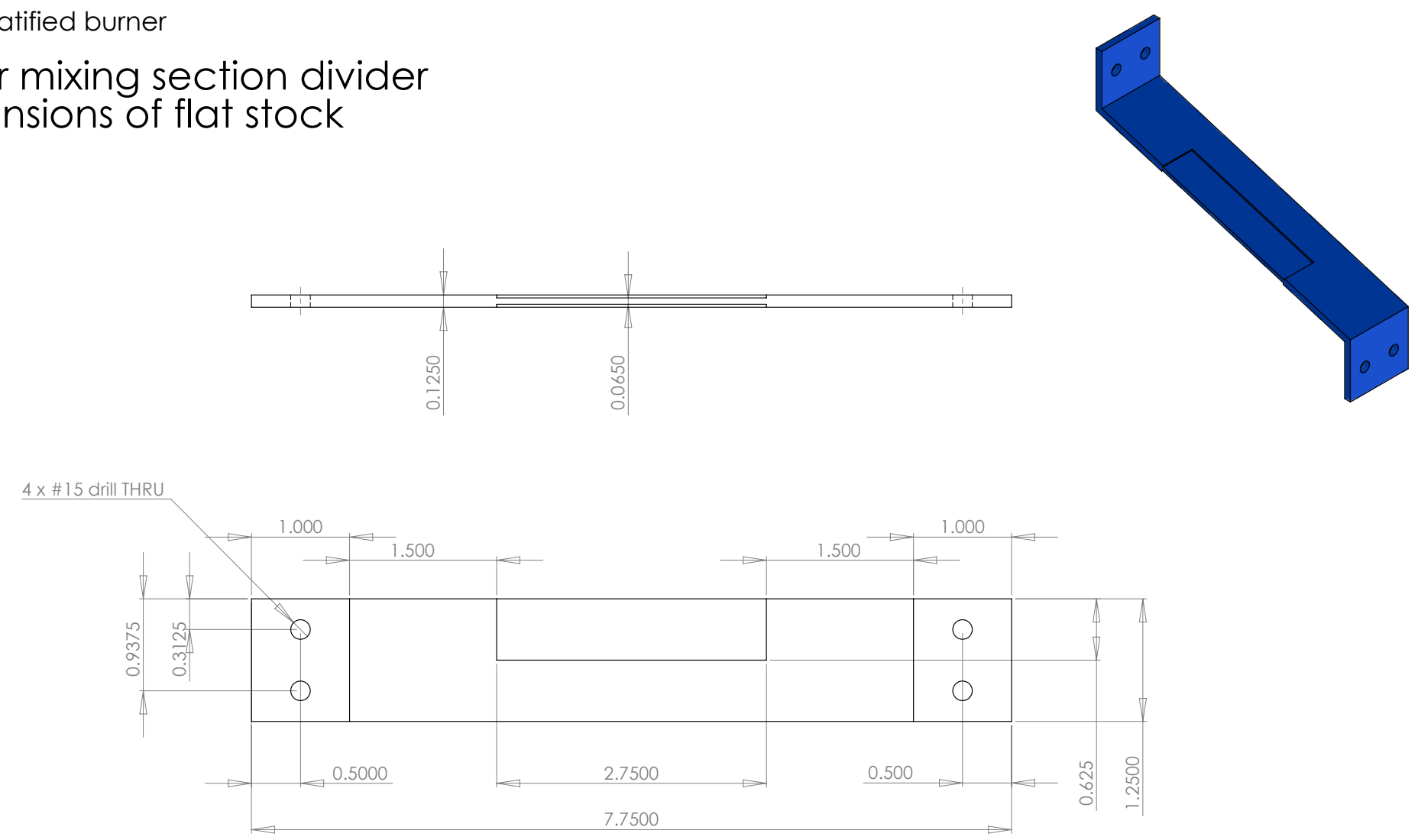

MATERIAL : aluminum

ALL DIMENSIONS +/-0.005

1 OFF 
NRC stratified burner

lower mixing section top

side $A$, hole details
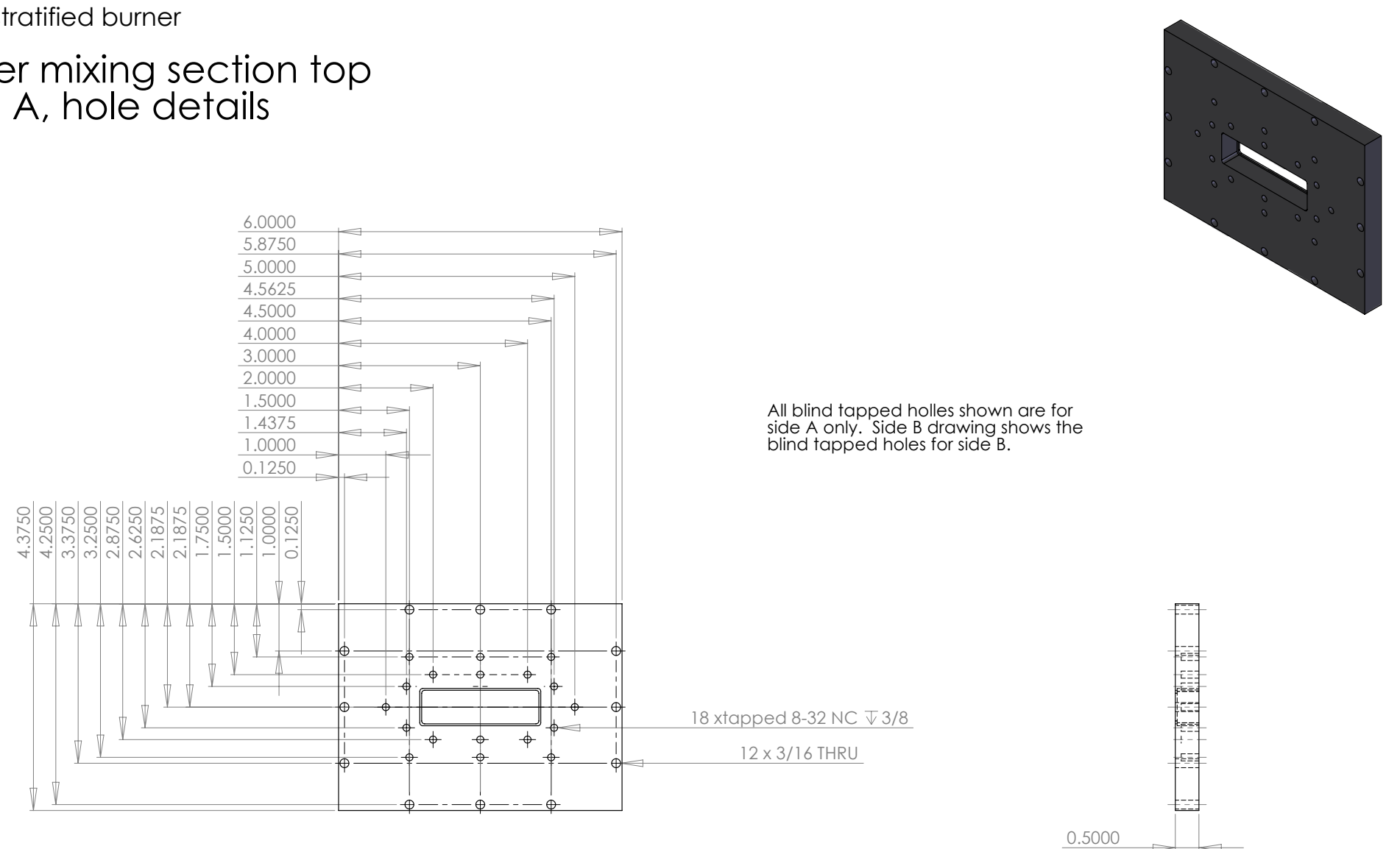

MATERIAL : aluminum

ALL DIMENSIONS +/-0.005

1 OFF 
NRC stratified burner

lower mixing section top

side A, milled slot details

corner fillets to be 0.0625 radius

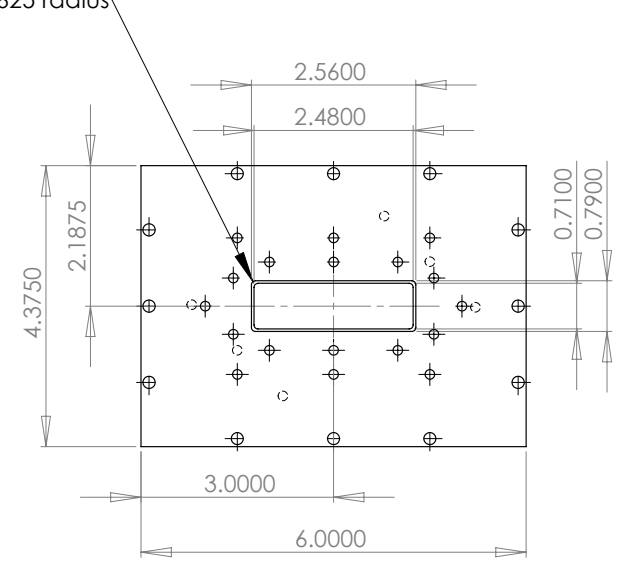

MATERIAL : aluminum

ALL DIMENSIONS +/-0.005
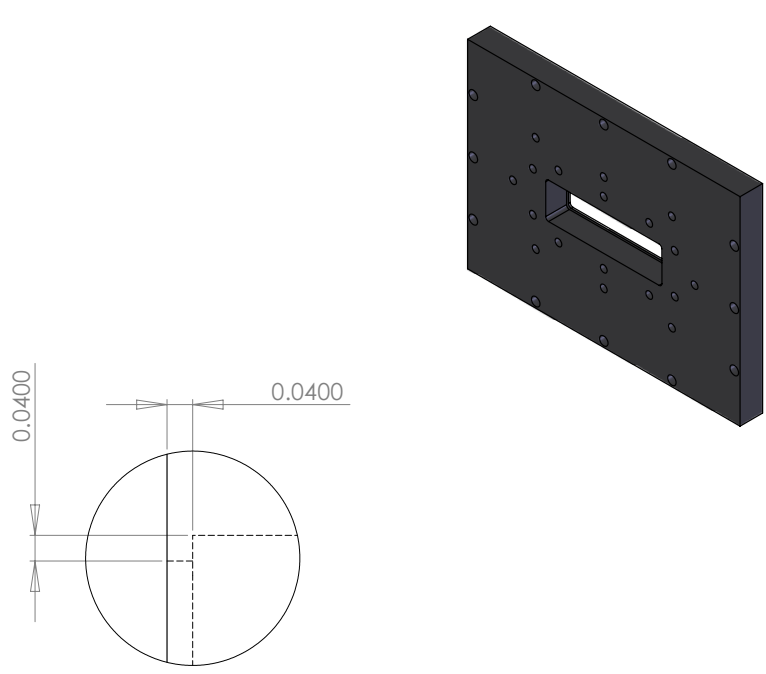

DETAIL milled slot

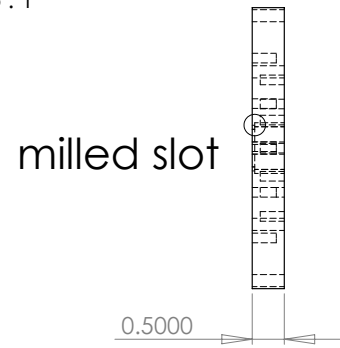


NRC stratified burner

lower mixing section top

side $B$, hole details

All blind tapped holles shown are for side B only. Side A drawing shows the blind tapped holes for side A.
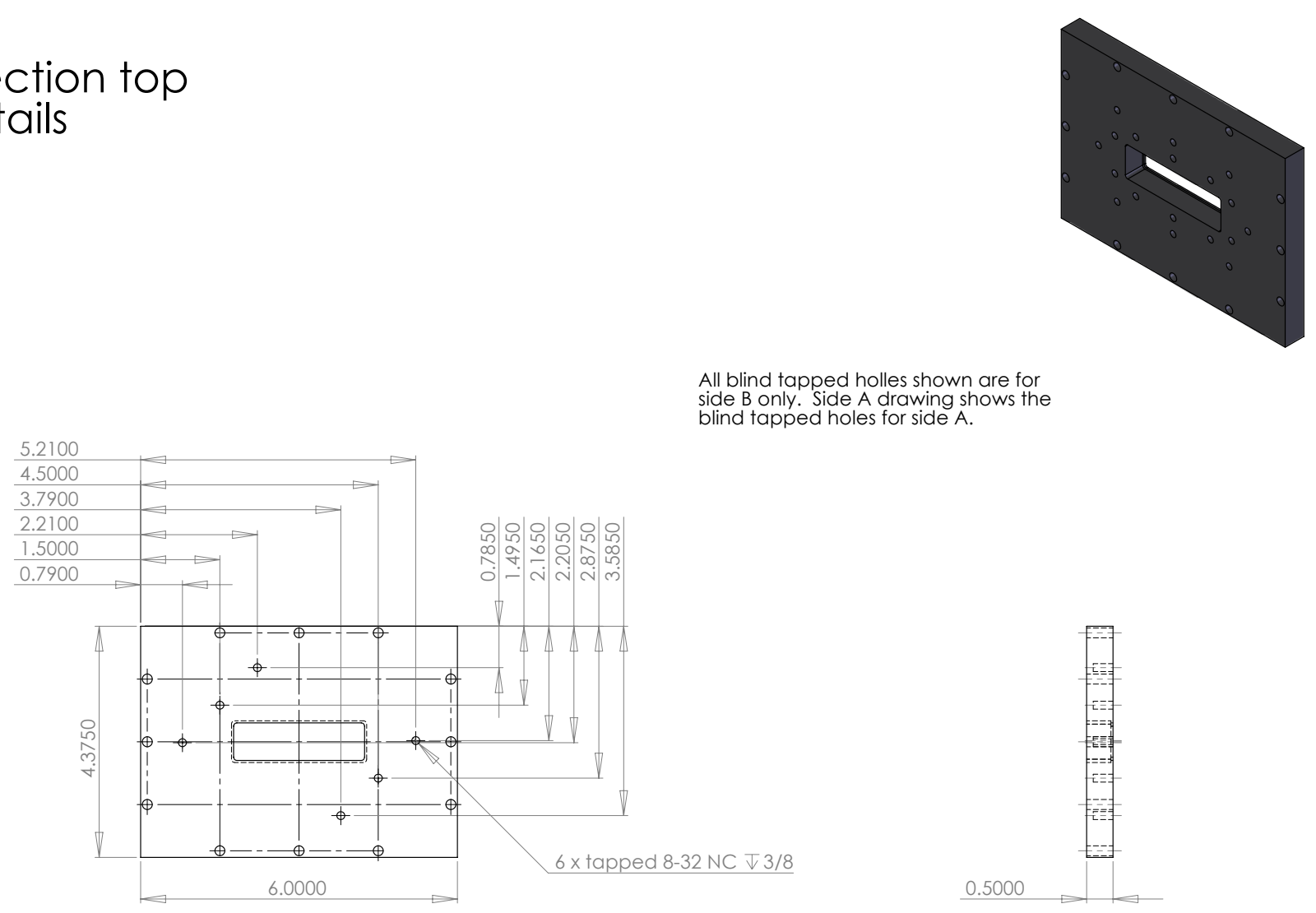

MATERIAL : aluminum

ALL DIMENSIONS +/-0.005

$1 \mathrm{OFF}$ 
NRC stratified burner

lower mixing section flow control plate
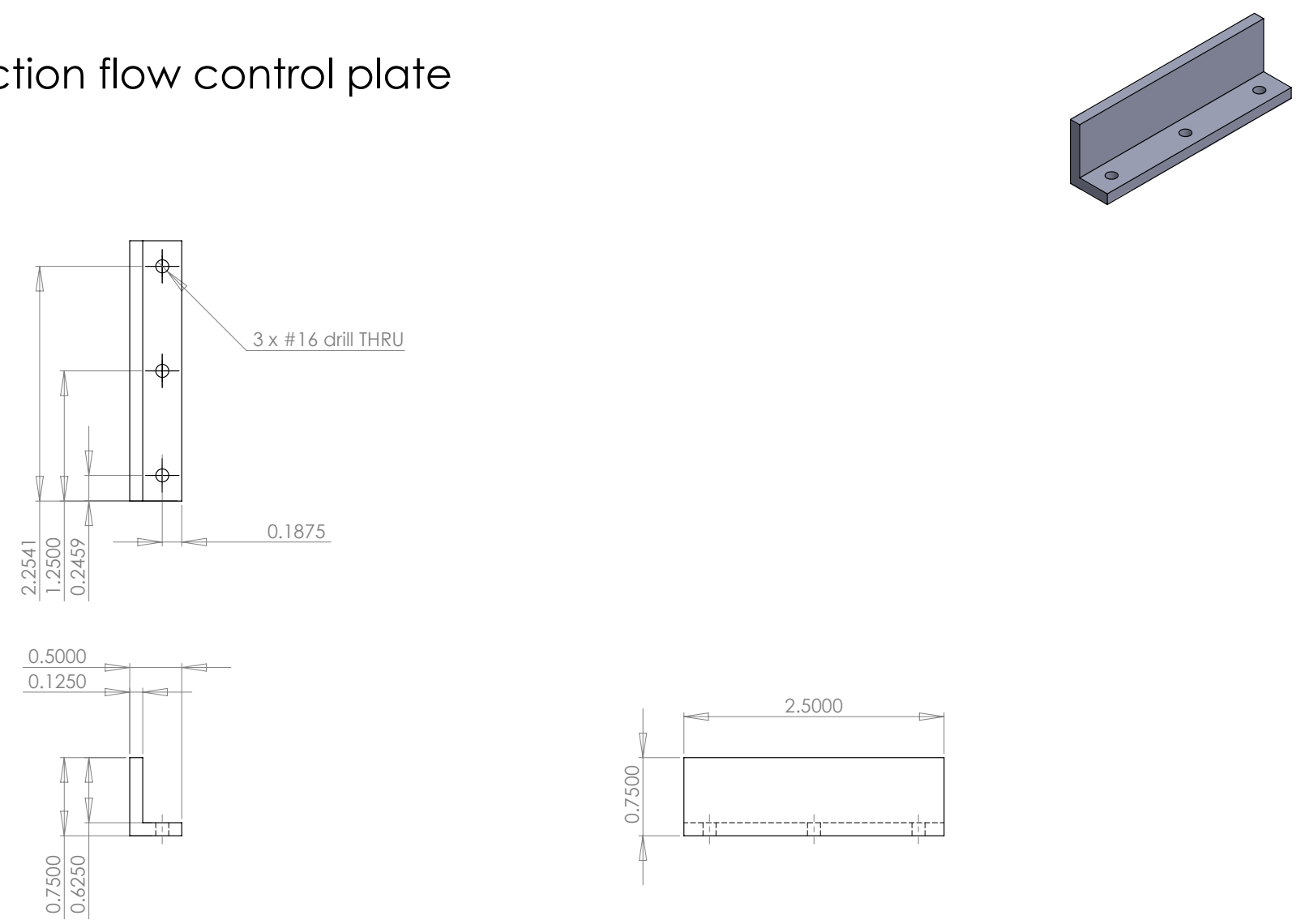

MATERIAL : aluminum

ALL DIMENSIONS +/-0.005

2 OFF 
NRC stratified burner

lower mixing section metal foam insert

For each unit make 2 wedges slightly longer and

wider than the drawing dimensions $(0.050)$. The user

complete face of the epoxied joint must act as a

barrier. Once the epoxy has cured the final matching
to sizes shown on the drawing can be done.
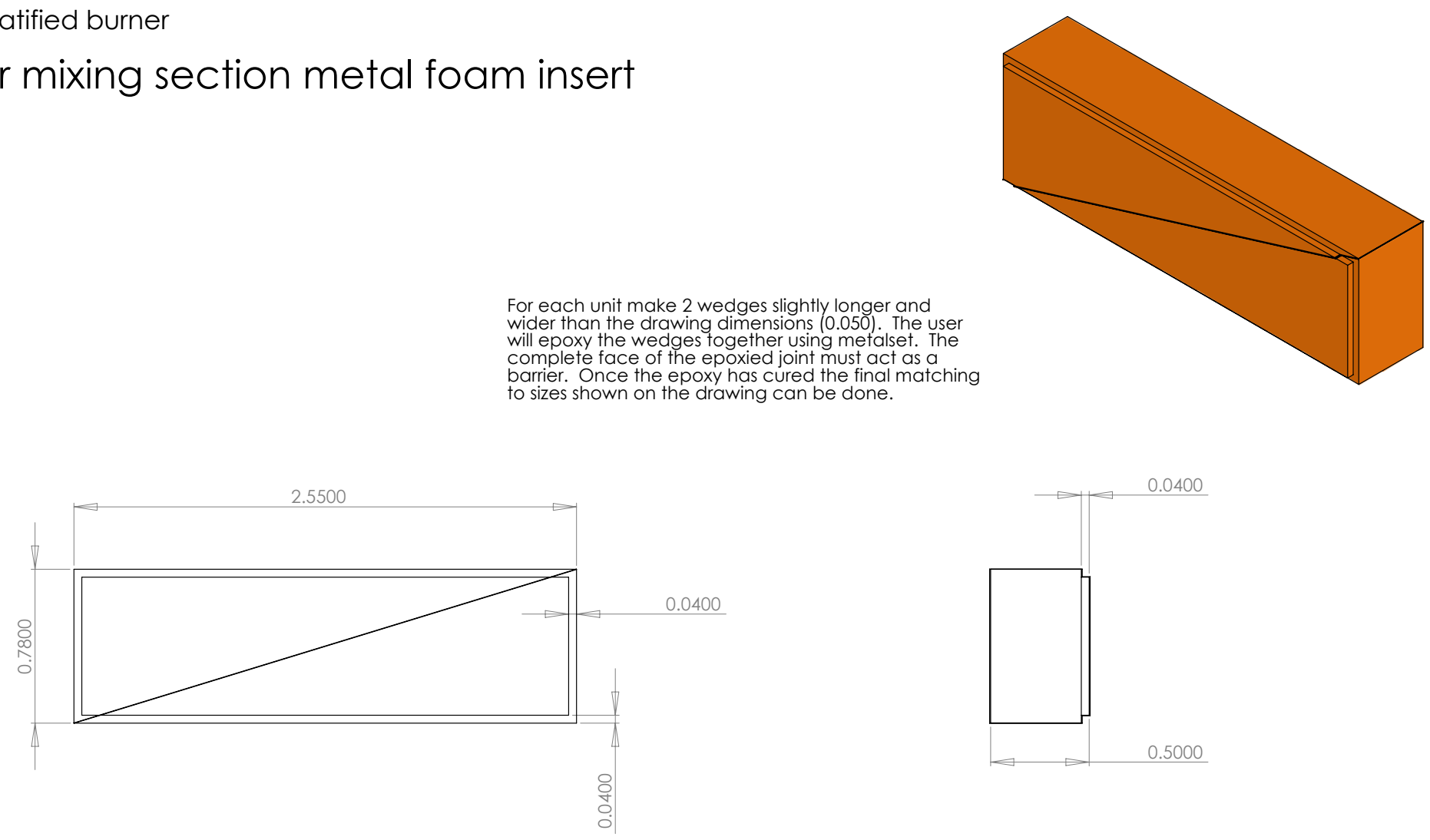

MATERIAL : aluminum

note: The metal foam supplied is soft and can be

ALL DIMENSIONS +/-0.005

1 OFF 
NRC stratified burner

lateral mixing section A

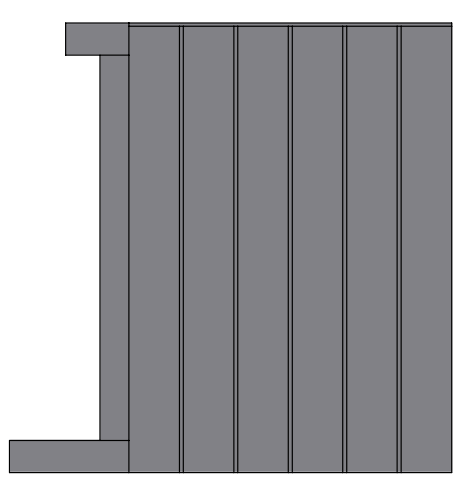

view $A$

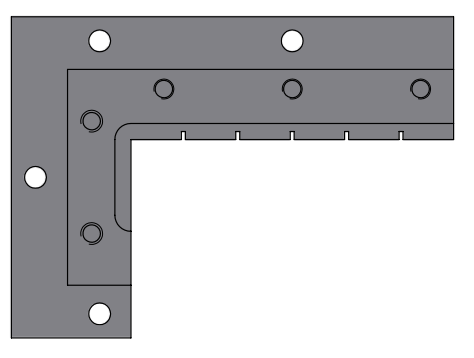

view $B$

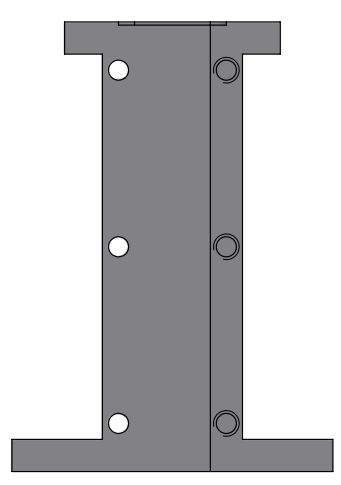

view C

MATERIAL : aluminum

ALL DIMENSIONS +/-0.005

1 OFF 
NRC stratified burner

lateral mixing section A view A

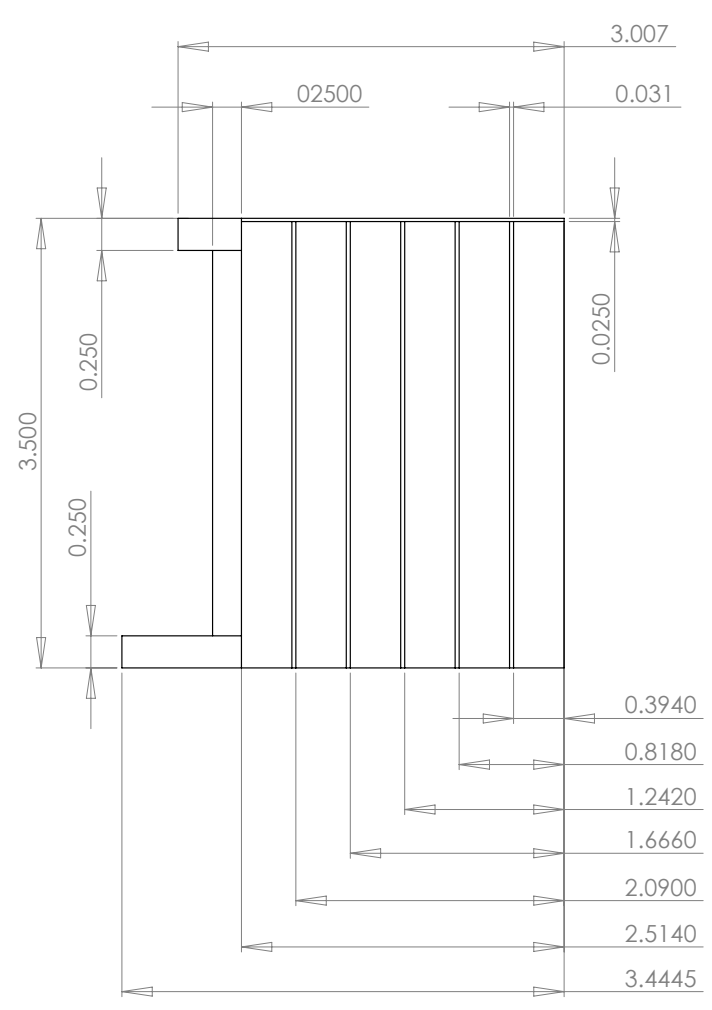

MATERIAL : aluminum

ALL DIMENSIONS +/-0.005

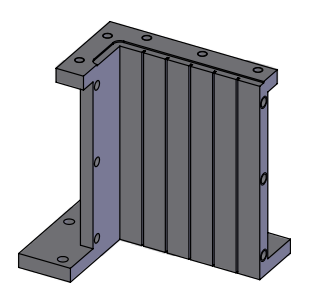

1 OFF 
NRC stratified burner

lateral mixing section $A$

view $B$, top holes
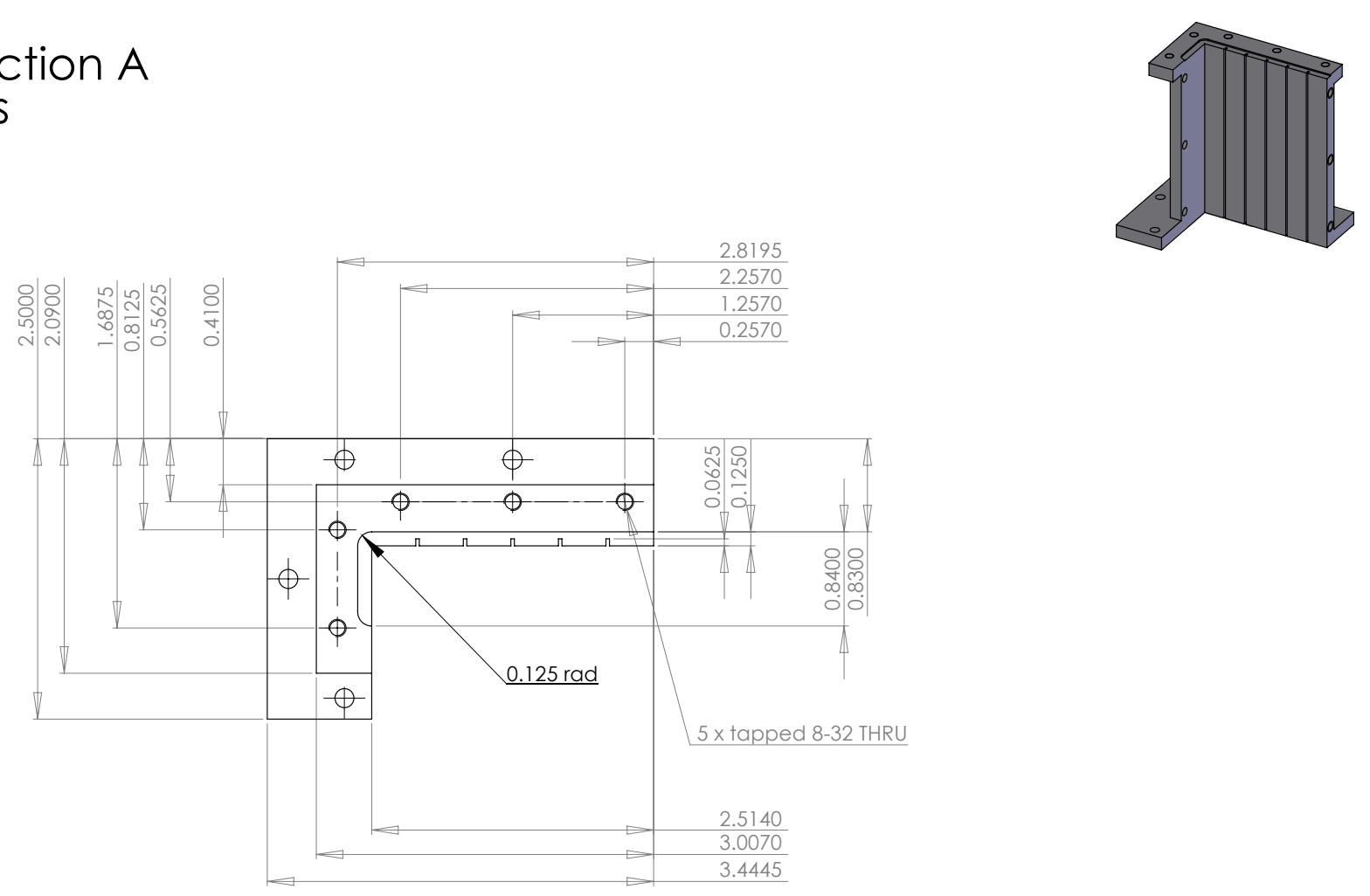

MATERIAL : aluminum

ALL DIMENSIONS +/-0.005

1 OFF 
NRC stratified burner

lateral mixing section $A$

view $B$, bottom holes
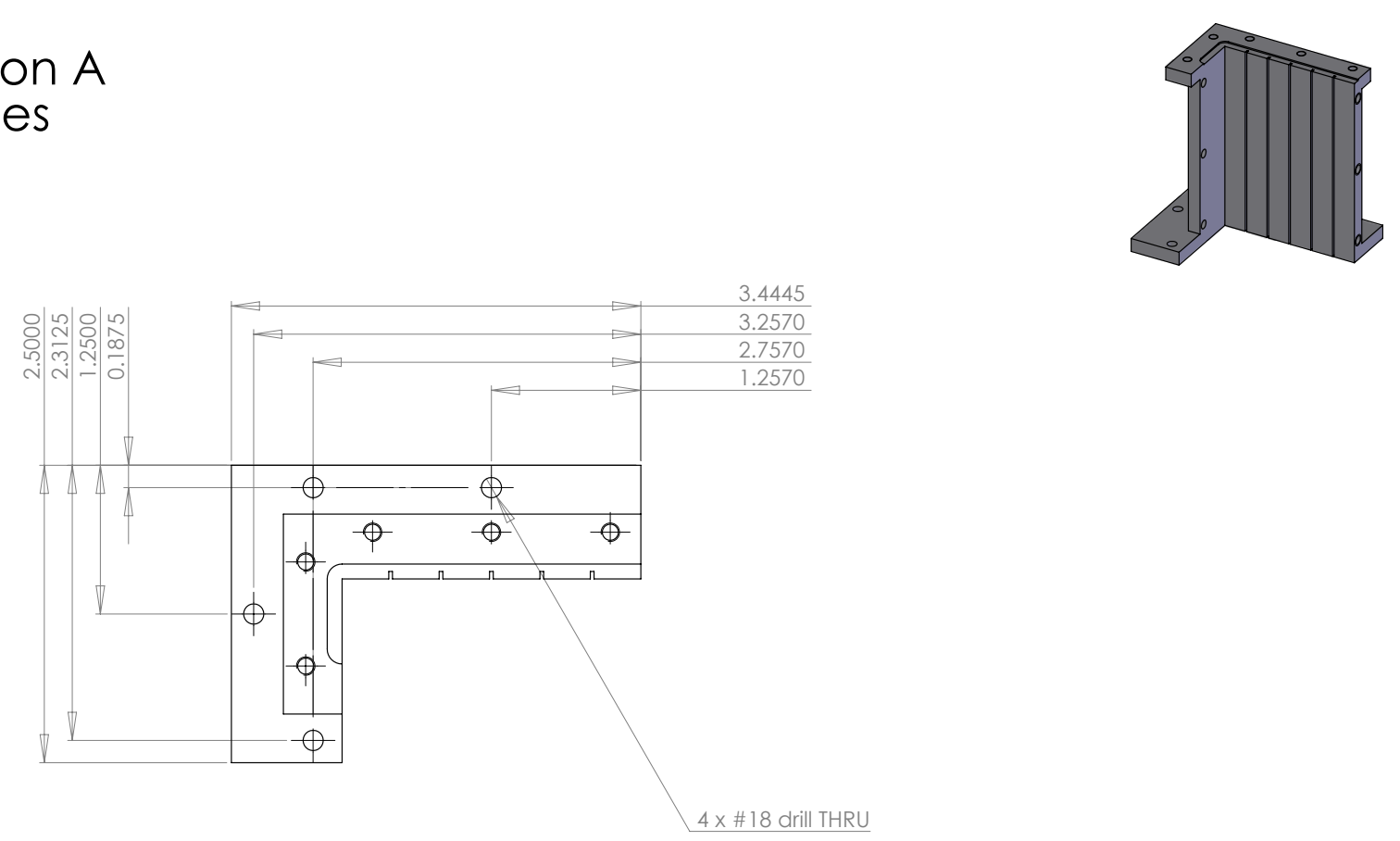

MATERIAL : aluminum

ALL DIMENSIONS +/-0.005

1 OFF 
NRC stratified burner

lateral mixing section $\mathrm{A}$ view $C$
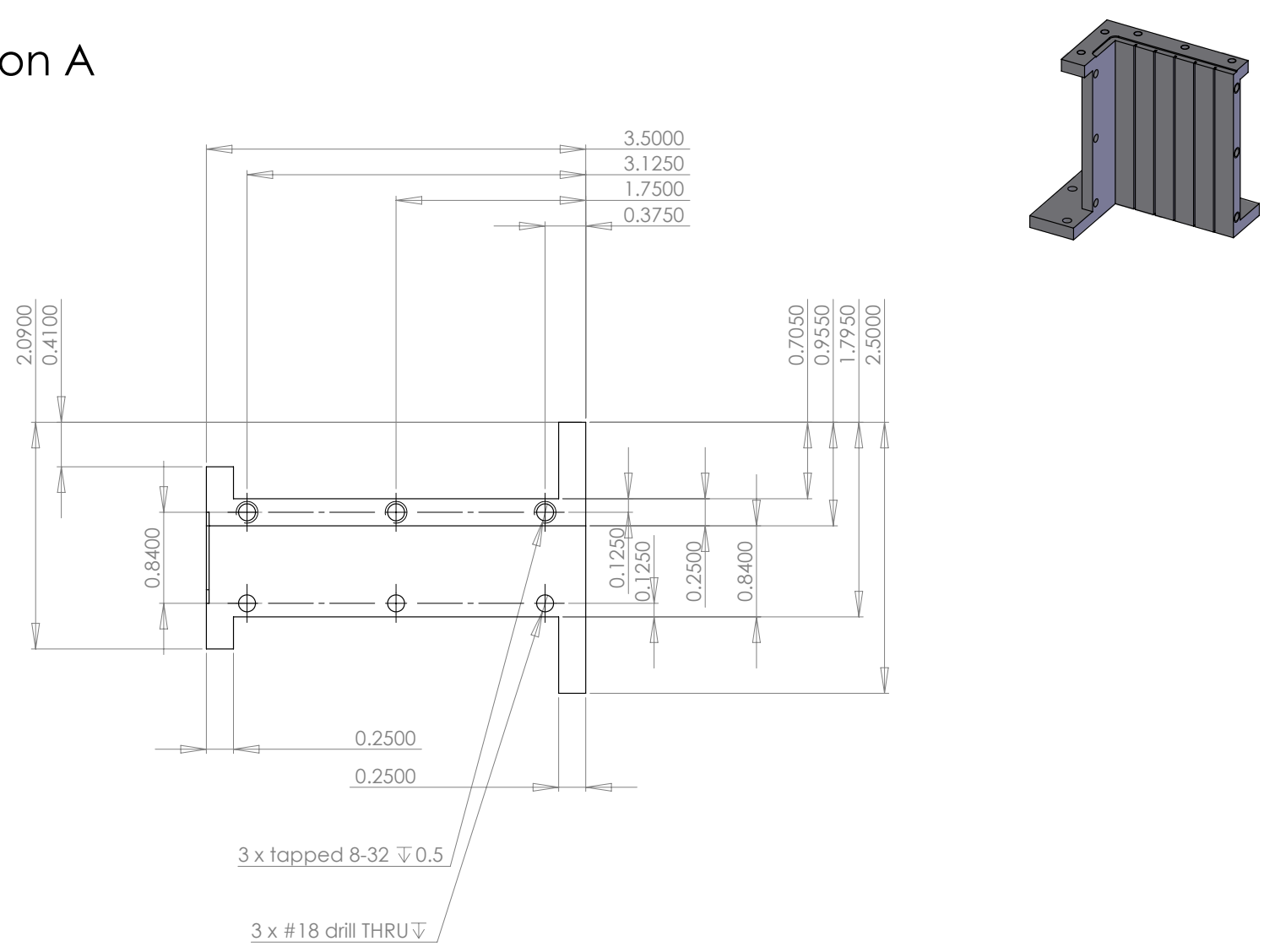

MATERIAL : aluminum

ALL DIMENSIONS +/-0.005

$1 \mathrm{OFF}$ 
NRC stratified burner

lateral mixing section B

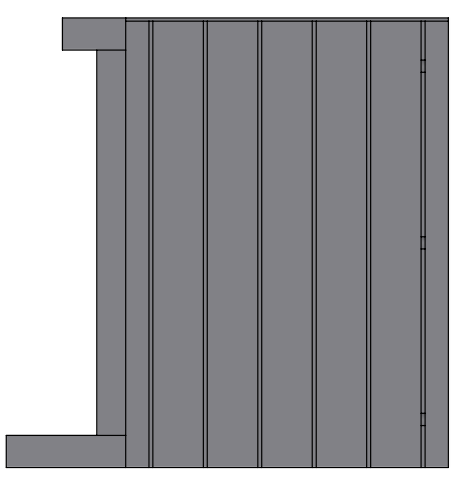

view $A$

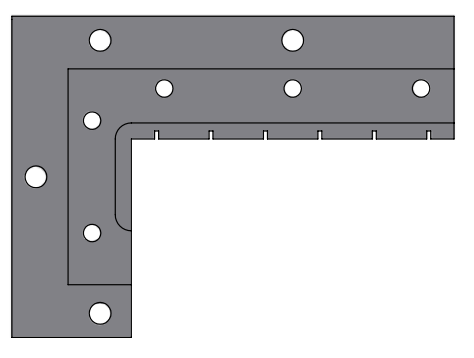

view $B$

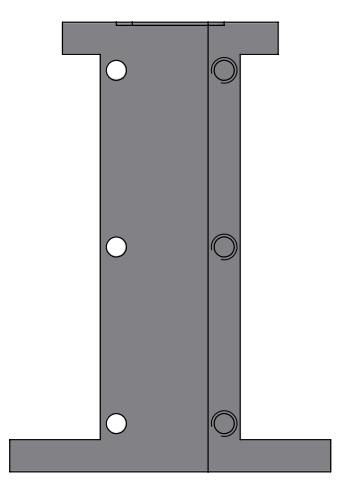

view C

MATERIAL : aluminum

ALL DIMENSIONS +/-0.005

1 OFF 
NRC stratified burner

lateral mixing section $B$ view $A$
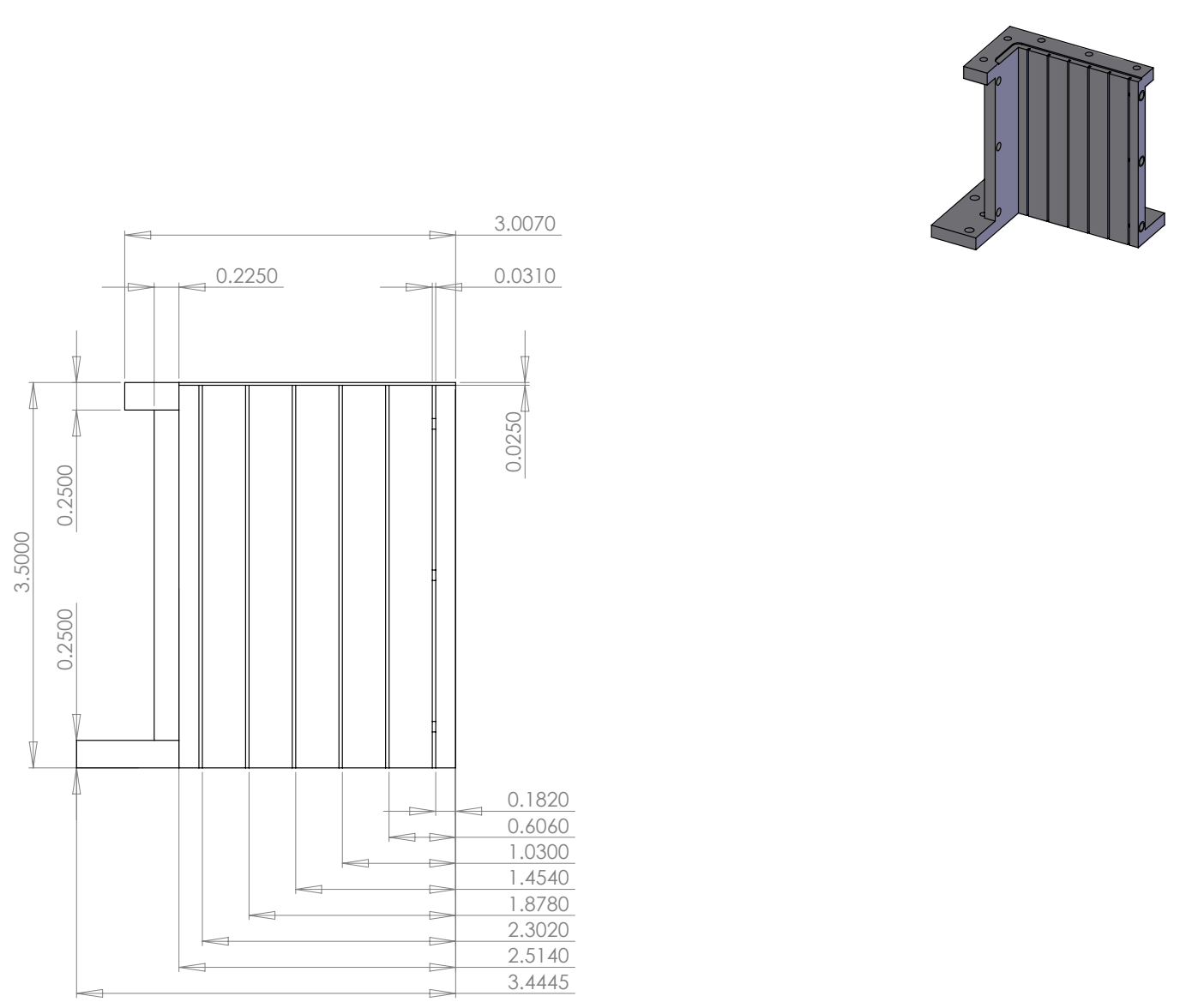

MATERIAL : aluminum

ALL DIMENSIONS +/-0.005

1 OFF 
NRC stratified burner

lateral mixing section $B$

view $B$, top holes
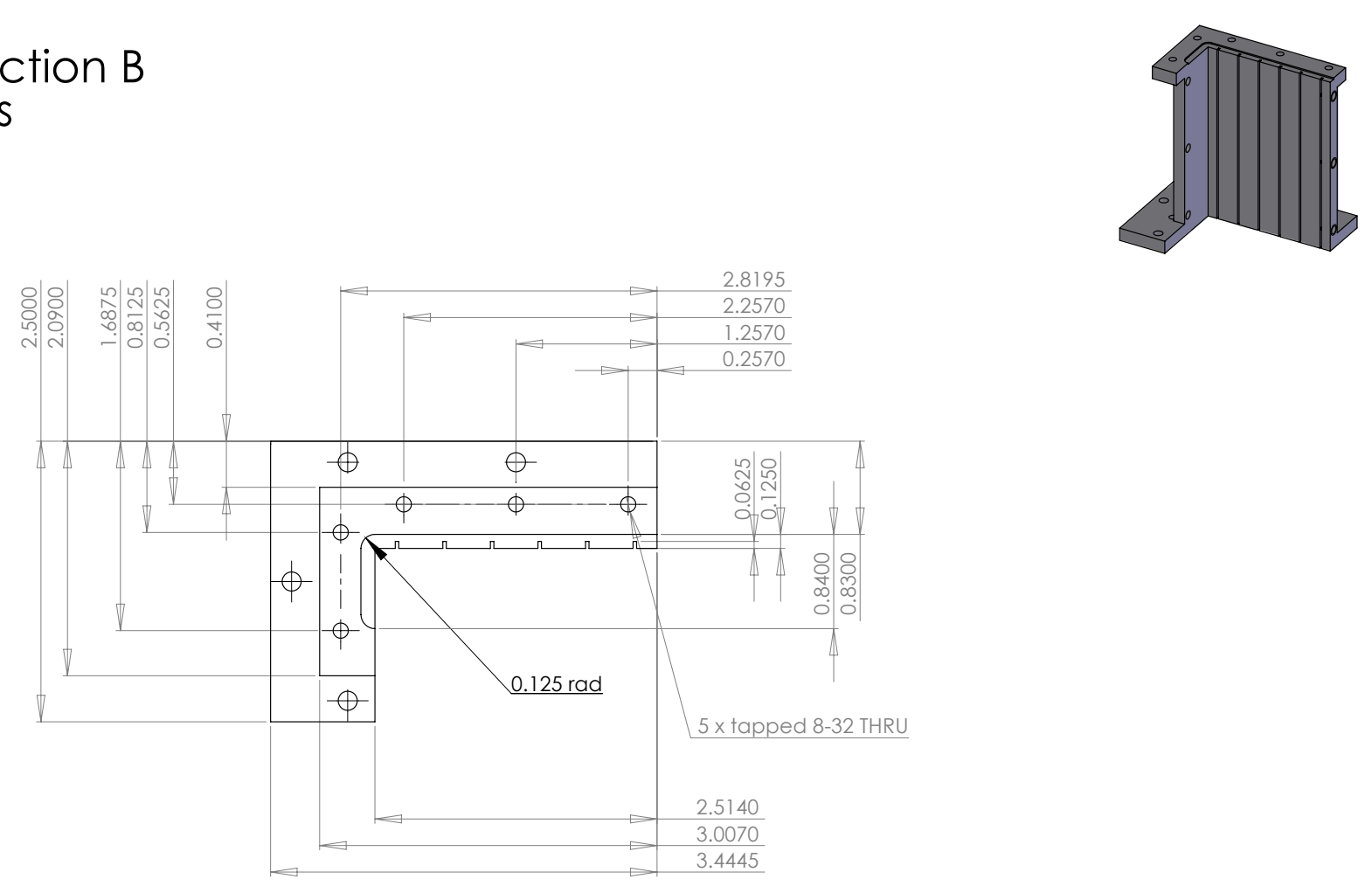

MATERIAL : aluminum

ALL DIMENSIONS +/-0.005

1 OFF 
NRC stratified burner

lateral mixing section $B$ view $B$, bottom holes
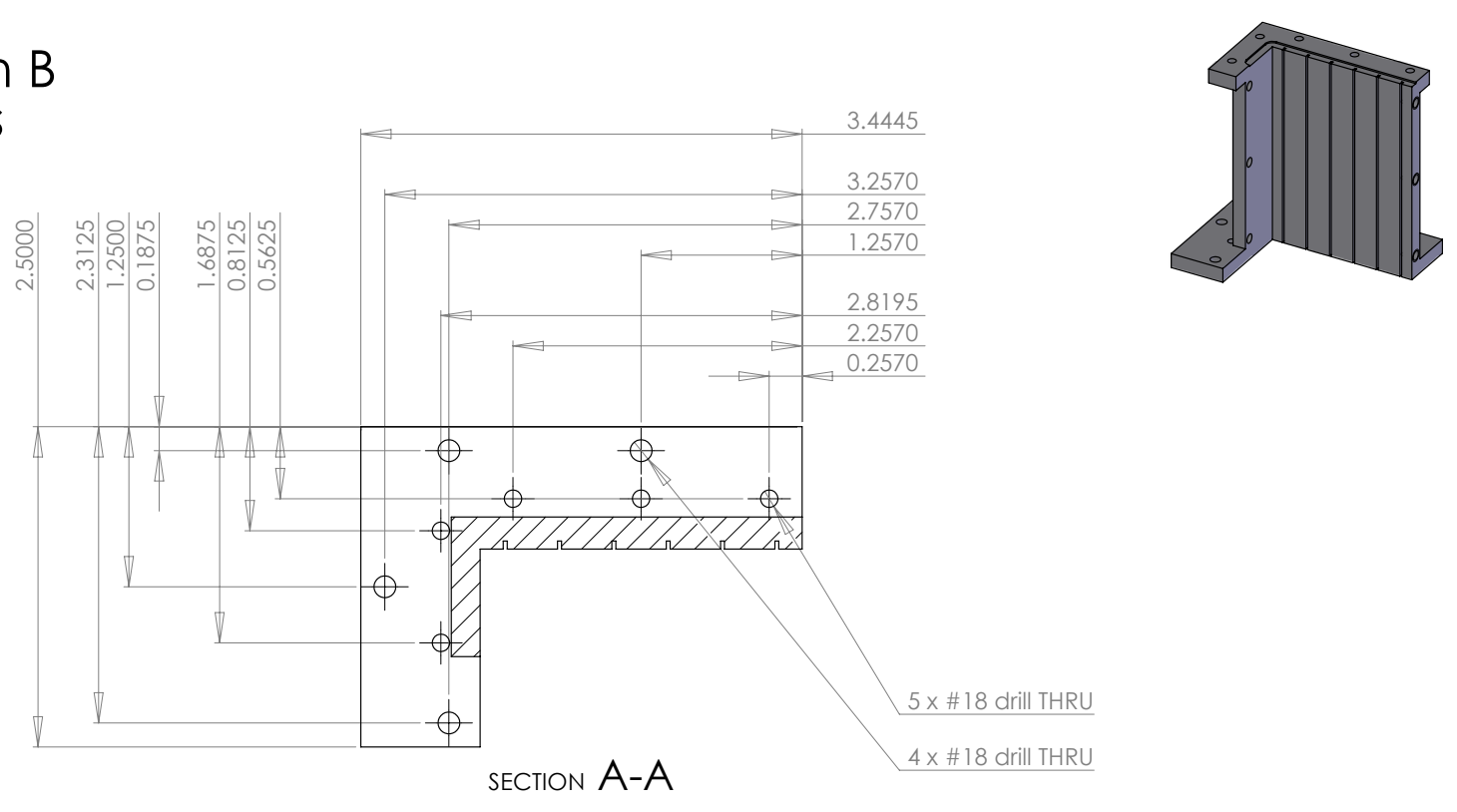

MATERIAL : aluminum

ALL DIMENSIONS +/-0.005

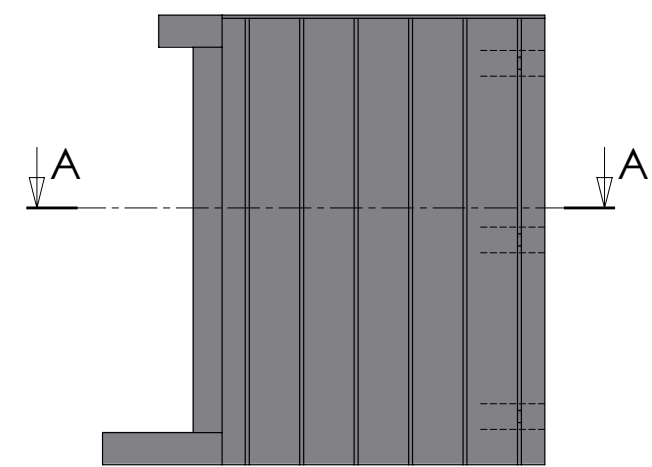

$1 \mathrm{OFF}$ 
NRC stratified burner

lateral mixing section $B$

view $C$
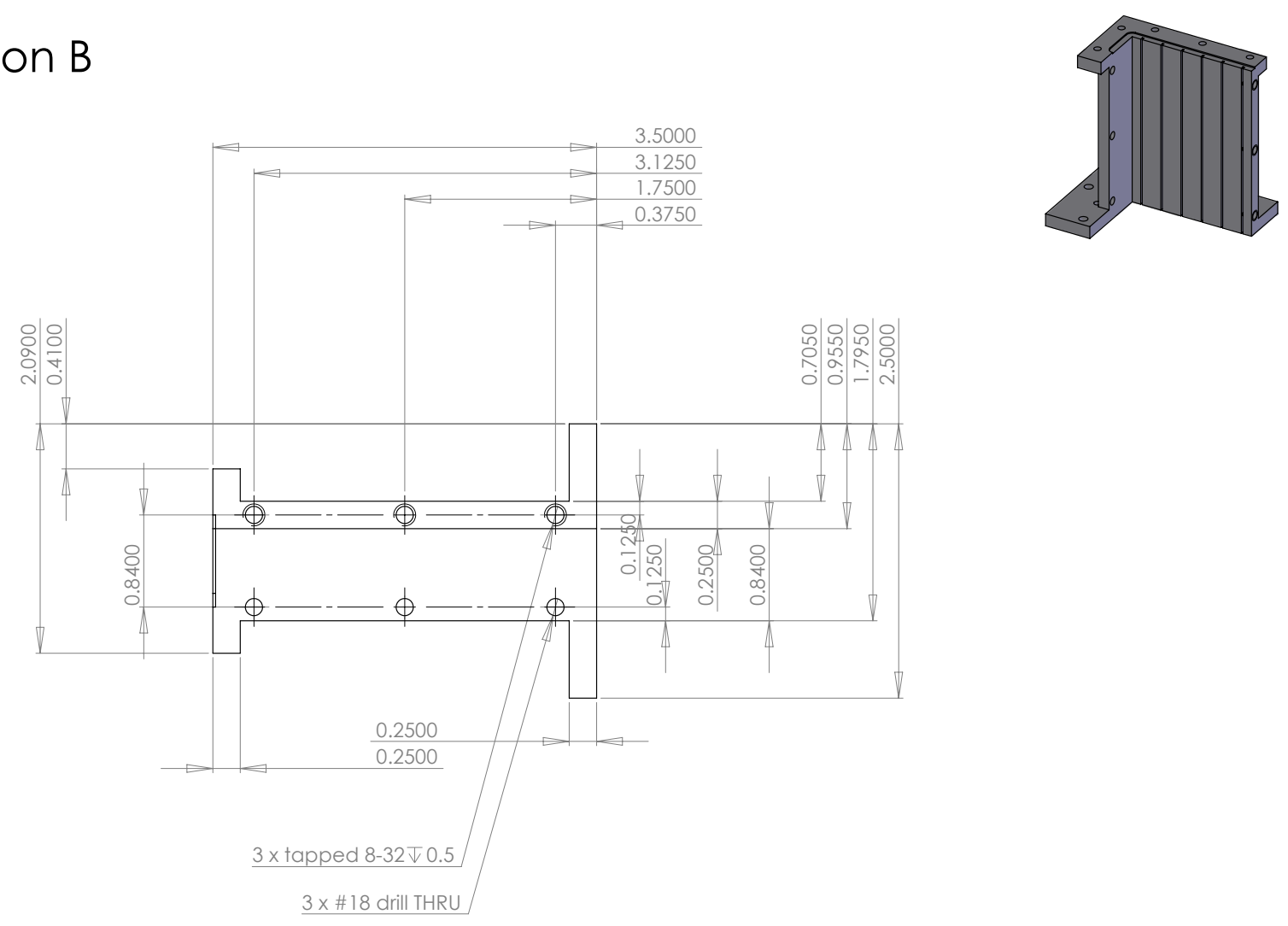

MATERIAL : aluminum

ALL DIMENSIONS +/-0.005

$1 \mathrm{OFF}$ 
NRC stratified burner

lateral mixing section divider


MATERIAL : aluminum

ALL DIMENSIONS +/-0.005

11 OFF 
NRC stratified burner

lateral mixing section screen
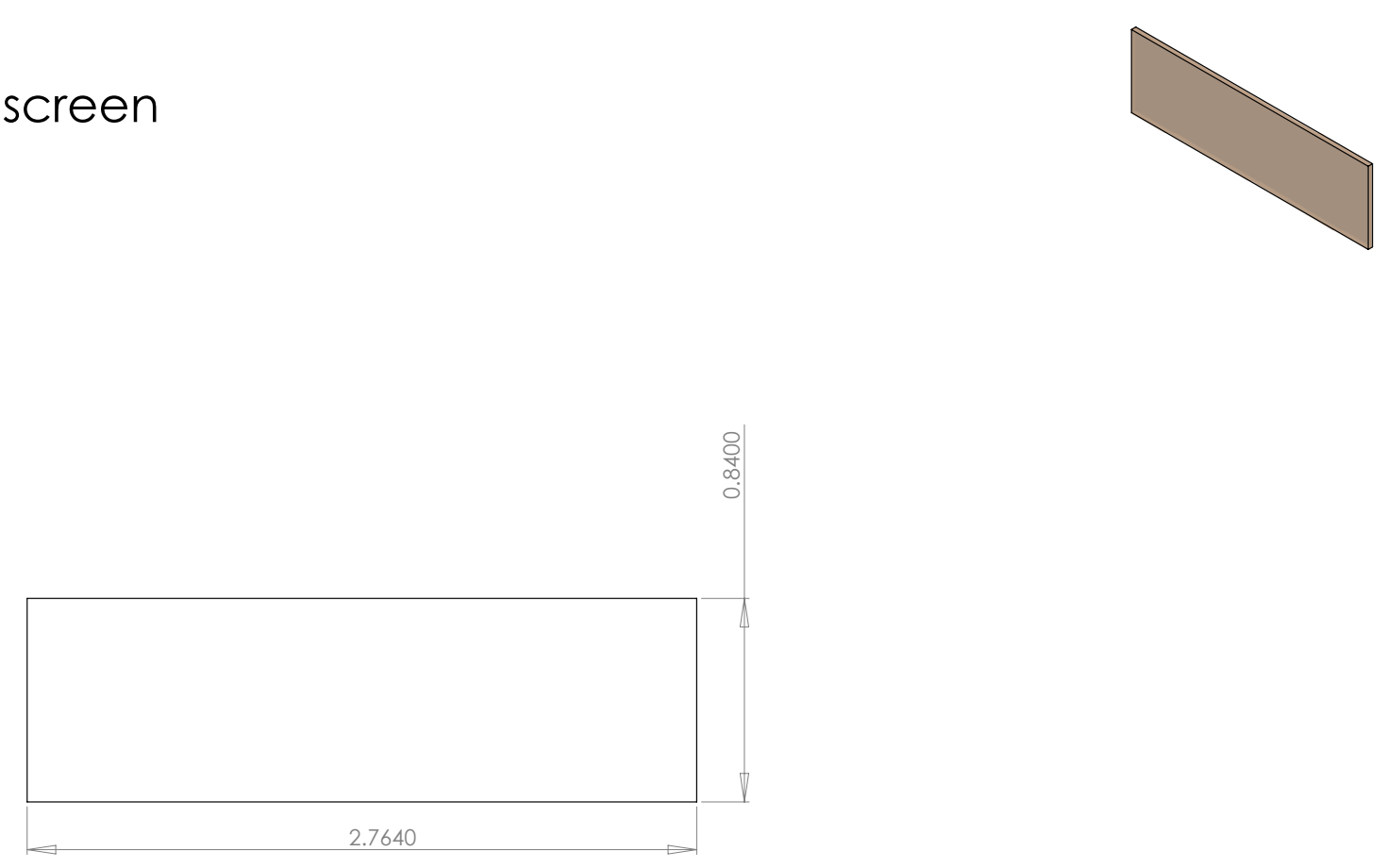

MATERIAL : stainless steel screen

ALL DIMENSIONS +/-0.005

2 OFF 
NRC stratified burner

lateral mixing section rubber gasket

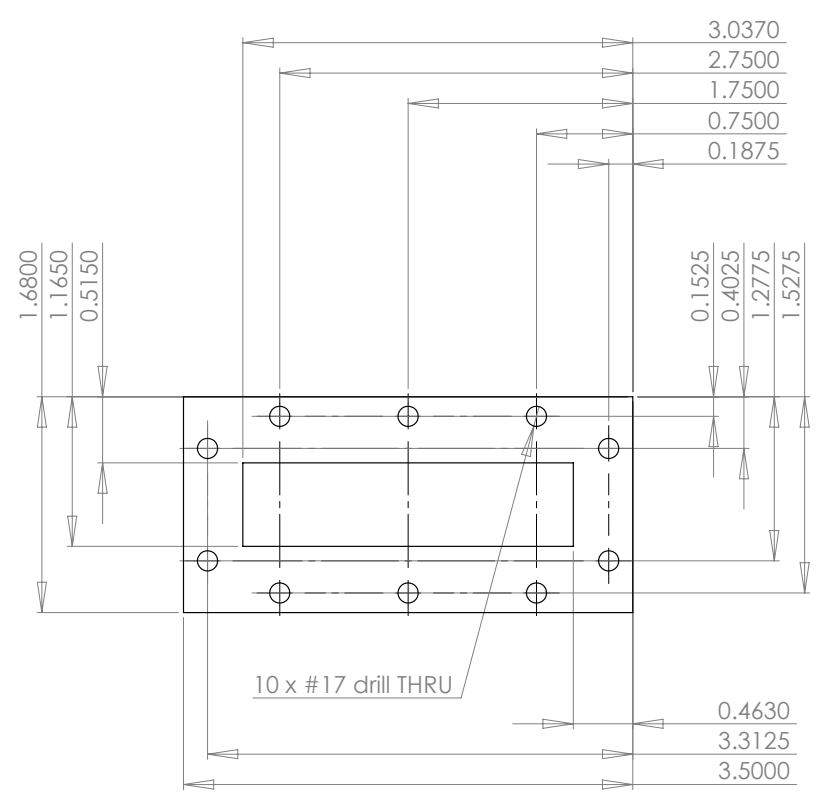

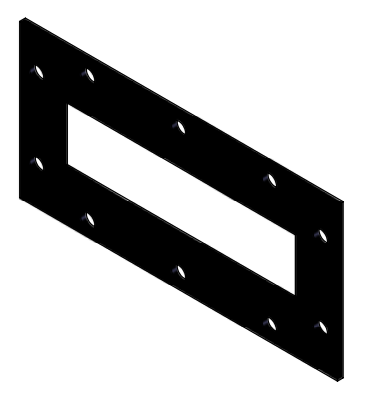

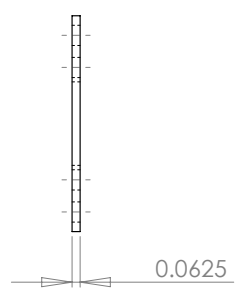

MATERIAL : rubber

ALL DIMENSIONS +/-0.005

1 OFF 
NRC stratified burner

transverse mixing section exit nozzle A
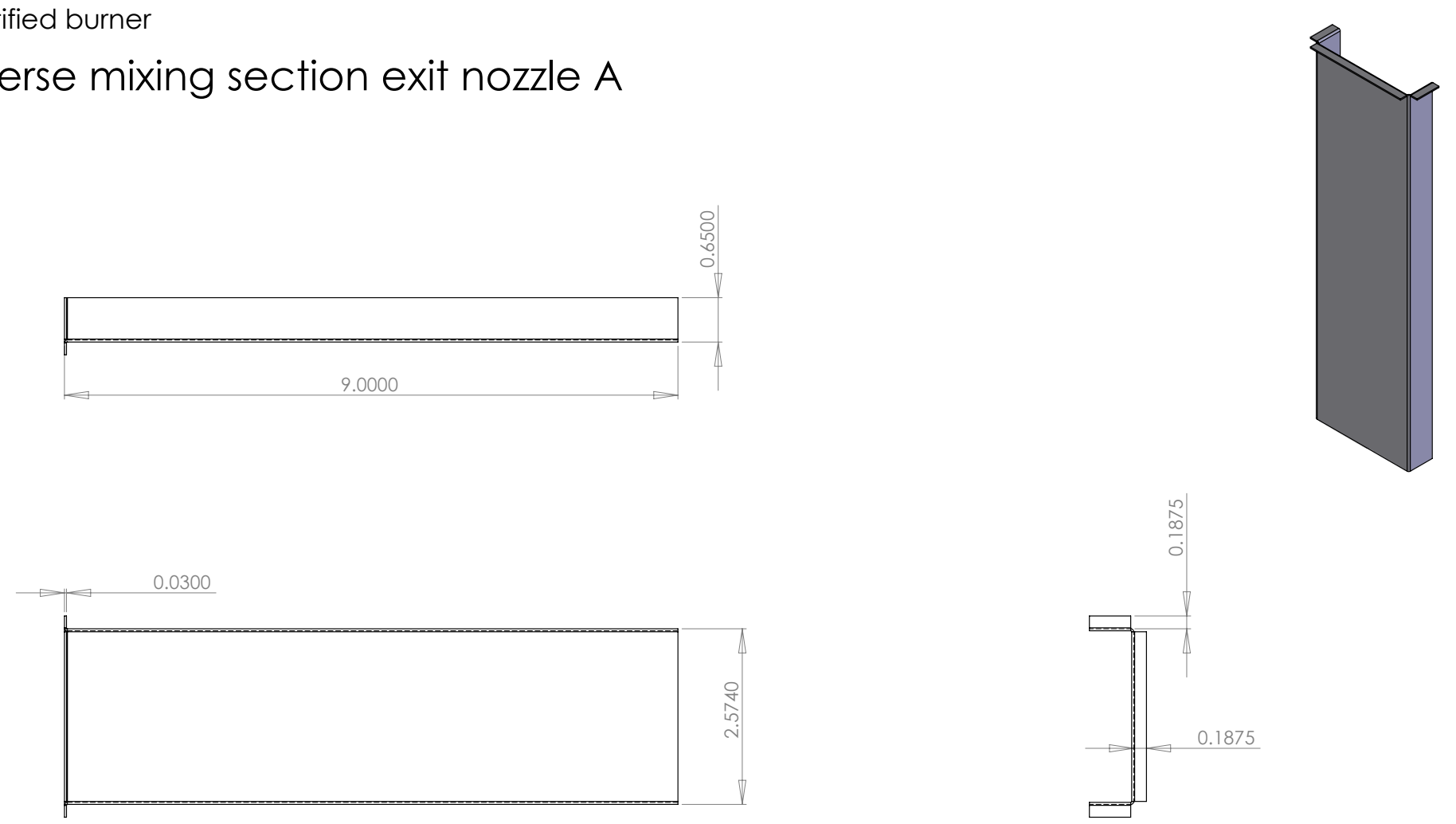

MATERIAL : 0.030 stainless steel

ALL DIMENSIONS +/-0.005

1 OFF 
NRC stratified burner

transverse mixing section exit nozzle $B$


MATERIAL : 0.030 stainless steel

ALL DIMENSIONS +/-0.005

1 OFF 
NRC stratified burner

transverse mixing section exit nozzle rubber gasket
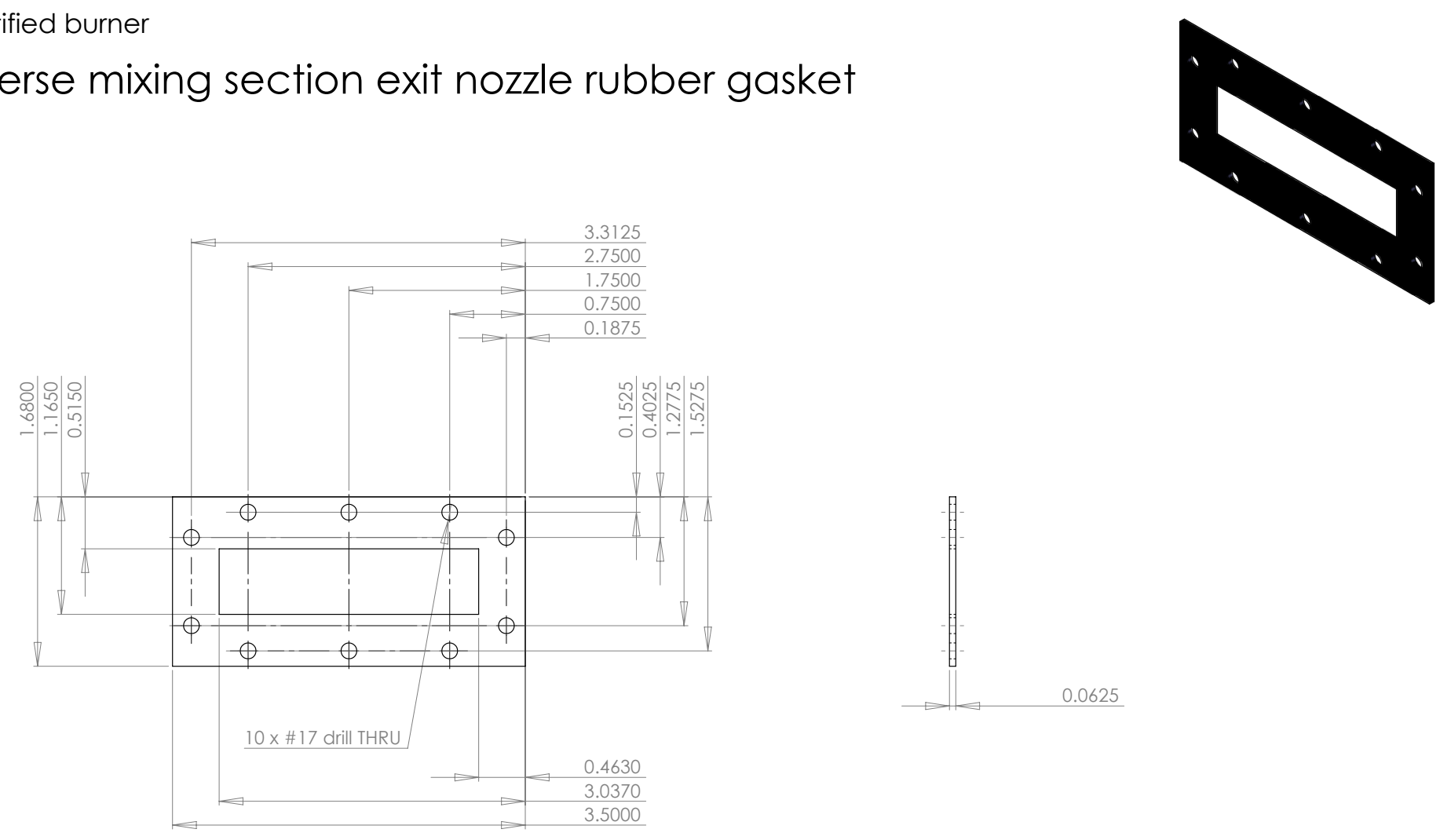

MATERIAL : rubber

ALL DIMENSIONS +/-0.005

1 OFF 
NRC stratified burner

co-flow base
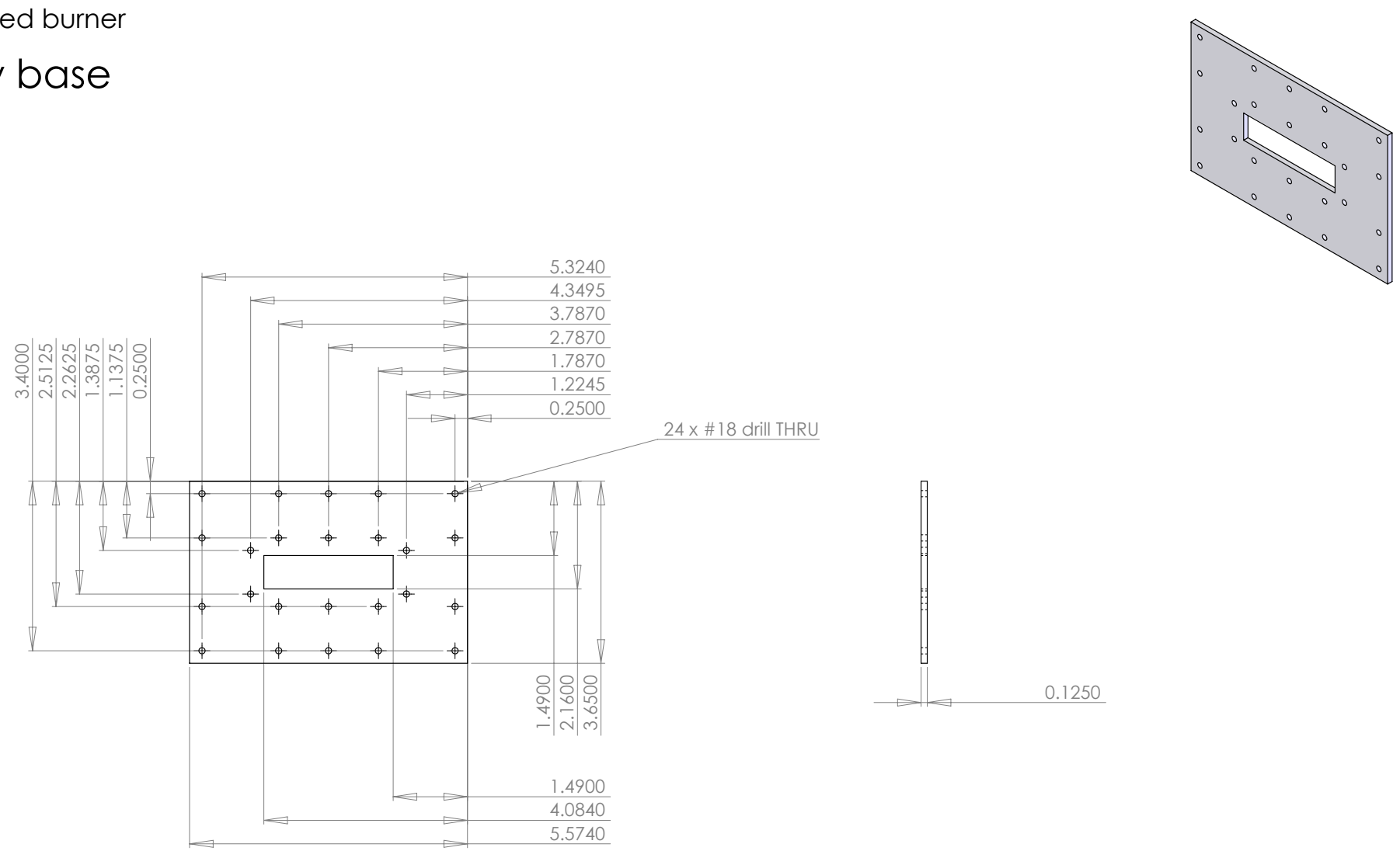

MATERIAL : aluminum

ALL DIMENSIONS +/-0.005

1 OFF 
NRC stratified burner

co-flow convergent nozzle rubber gasket
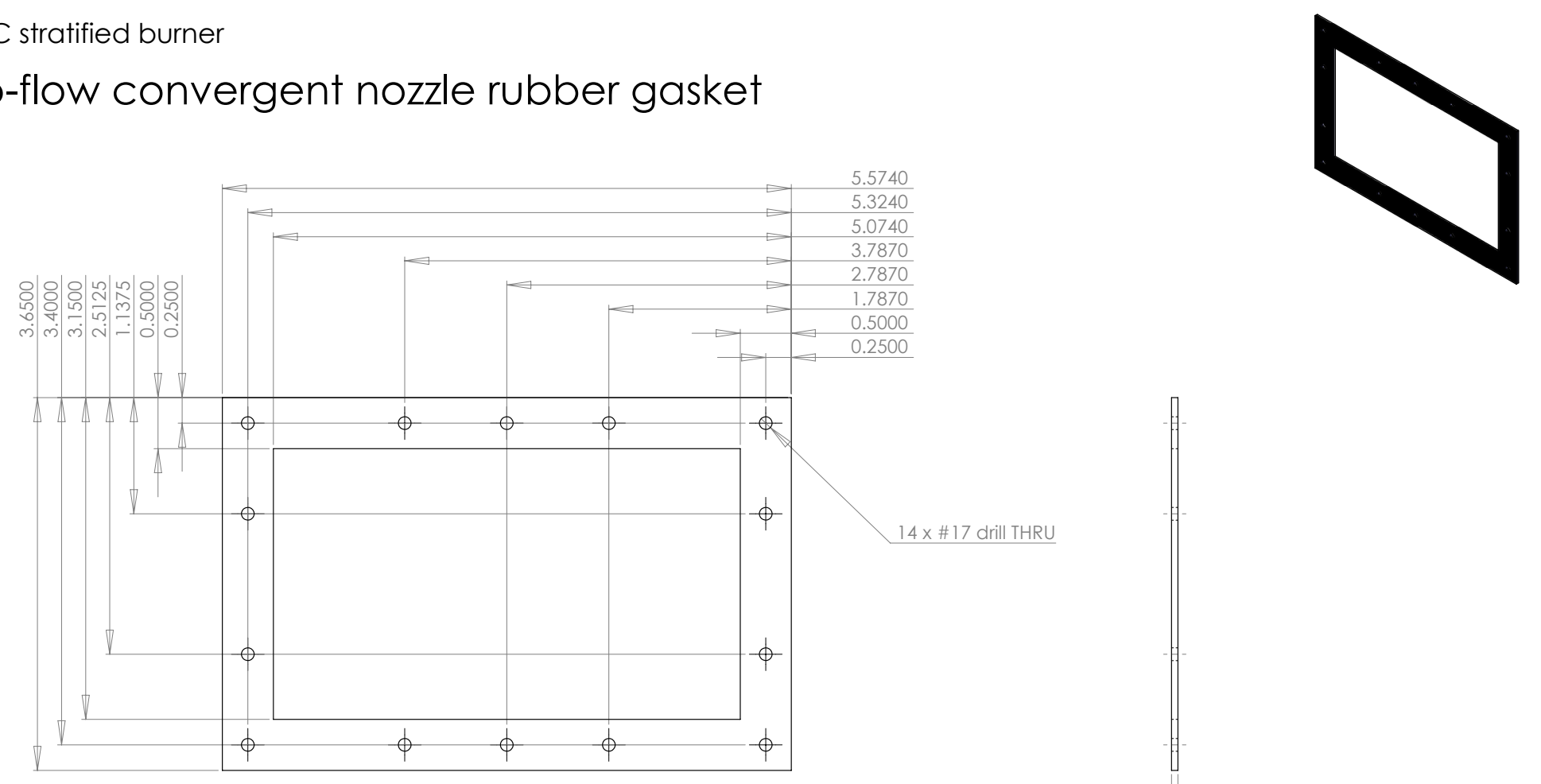

MATERIAL : rubber

ALL DIMENSIONS +/-0.005

1 OFF 
NRC stratified burner

co-flow convergent nozzle A
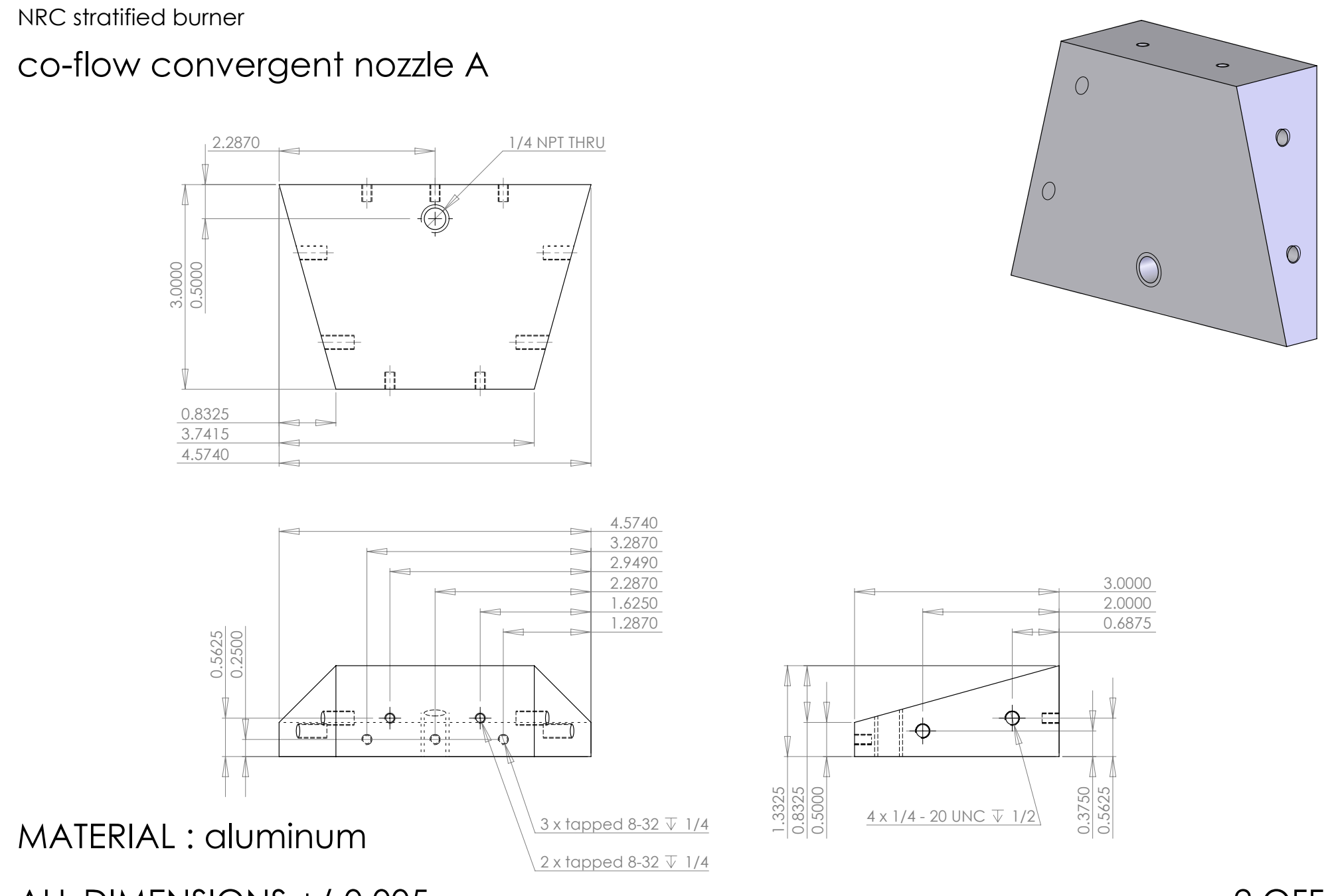

ALL DIMENSIONS +/-0.005

2 OFF 
NRC stratified burner

co-flow convergent nozzle B
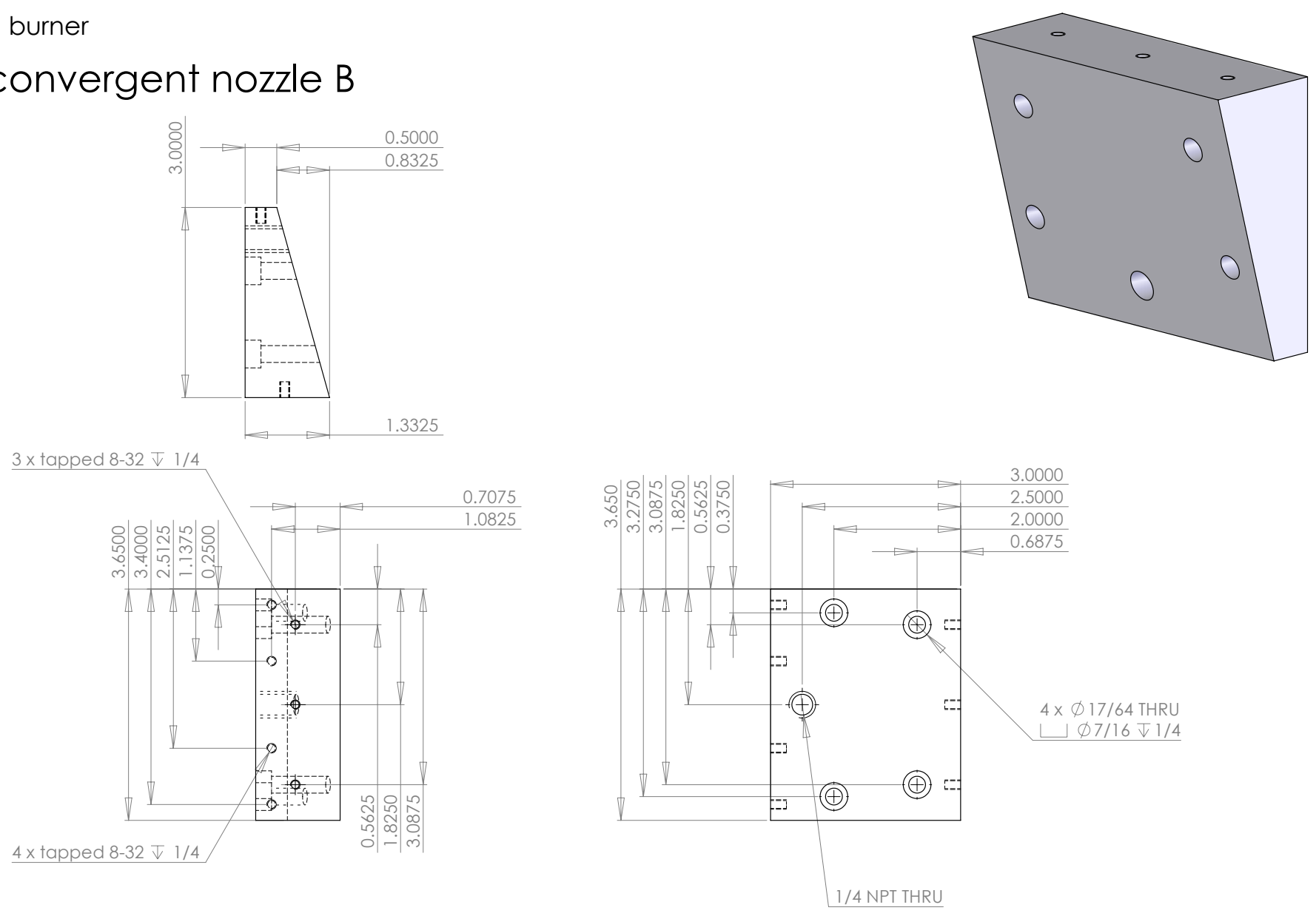

MATERIAL : aluminum

ALL DIMENSIONS +/-0.005

2 OFF 
NRC stratified burner

co-flow screen

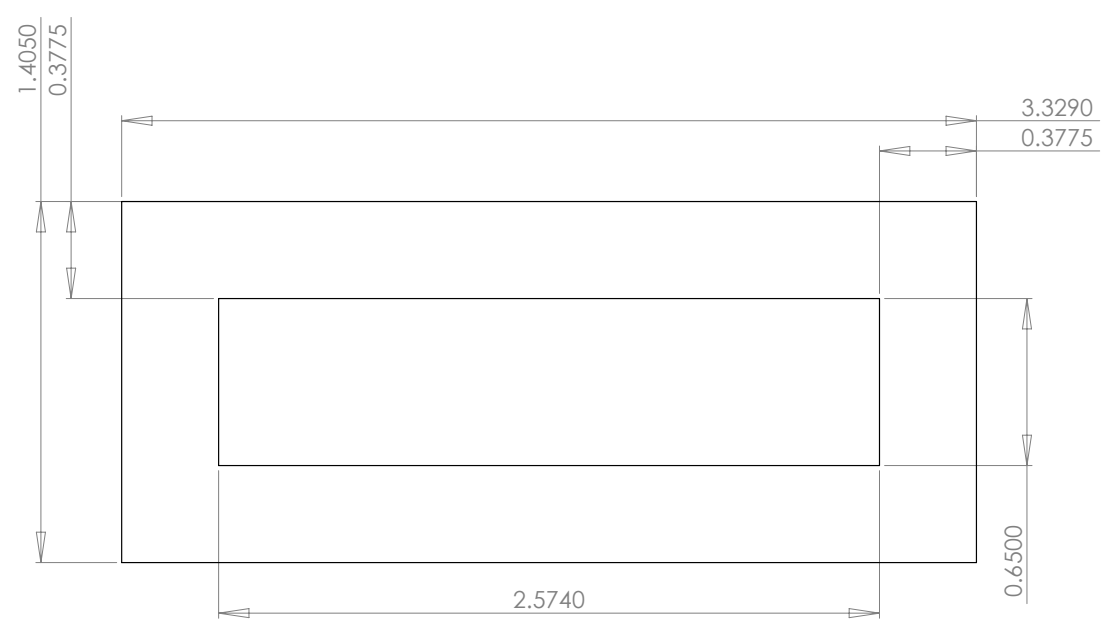

MATERIAL : stainless steel screen

ALL DIMENSIONS +/-0.005

$1 \mathrm{OFF}$ 
NRC stratified burner

co-flow exit nozzle A
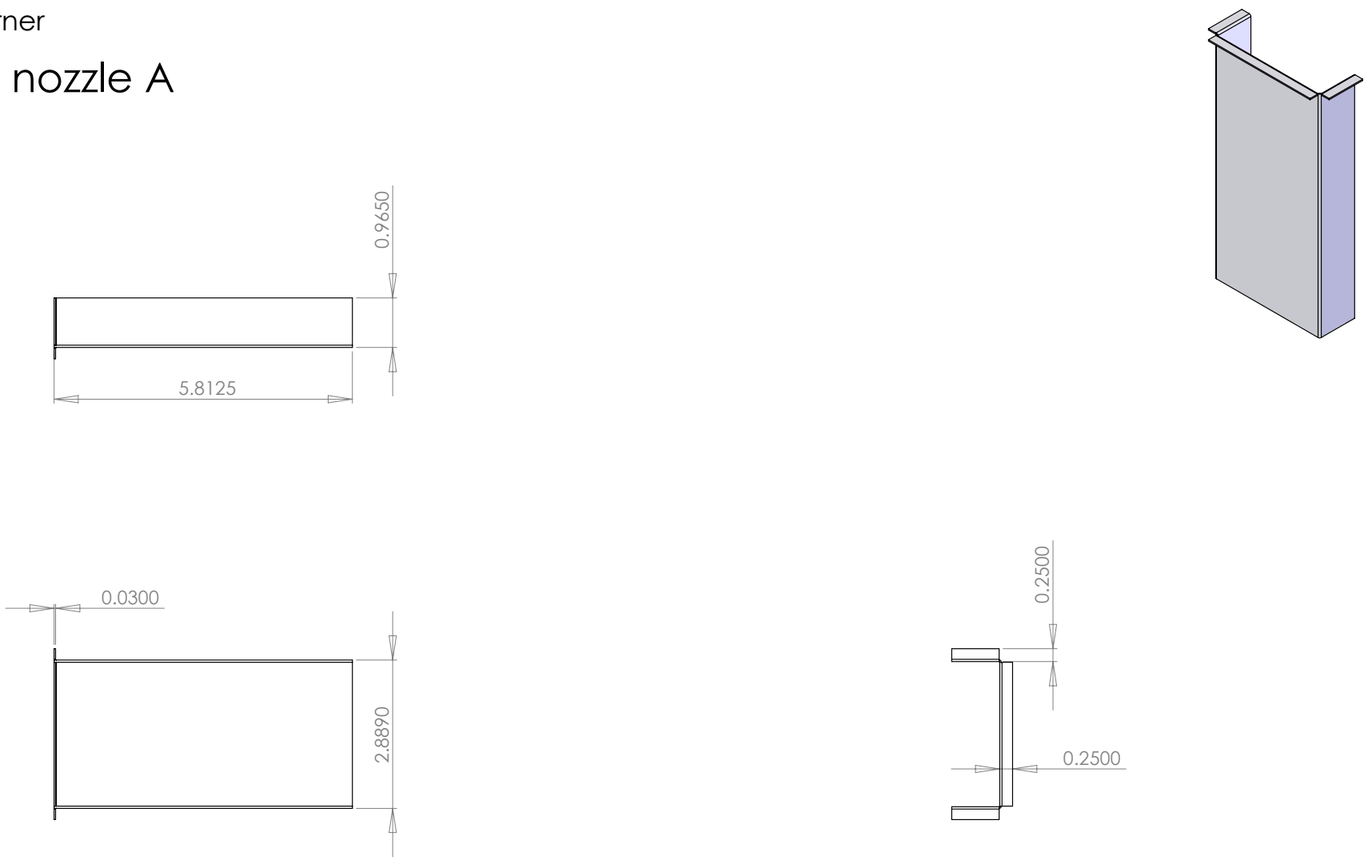

MATERIAL : 0.030 stainless steel

ALL DIMENSIONS +/-0.005

1 OFF 
NRC stratified burner

co-flow exit nozzle B
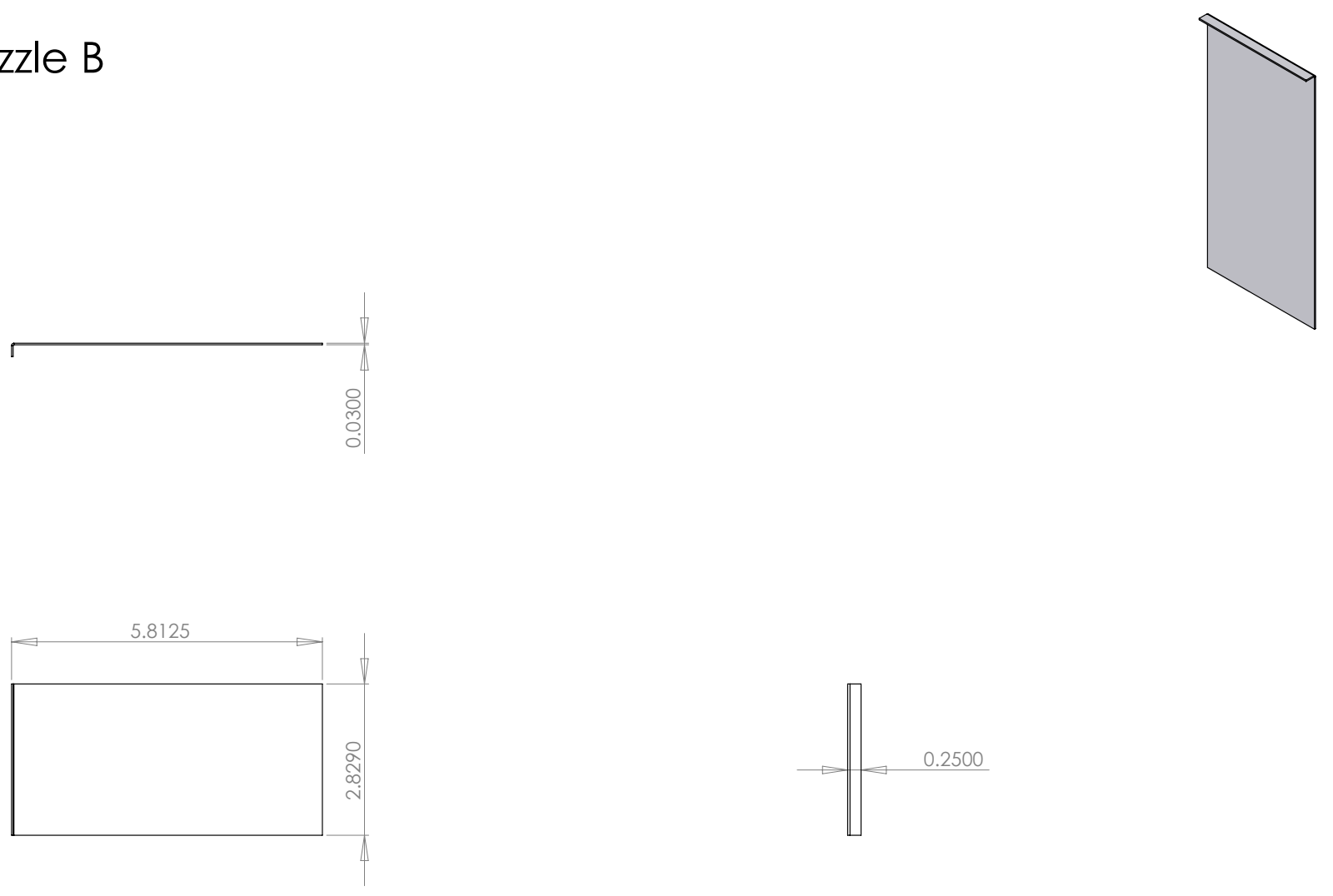

MATERIAL : 0.030 stainless steel

ALL DIMENSIONS +/-0.005

1 OFF 
NRC stratified burner

co-flow top rubber gasket
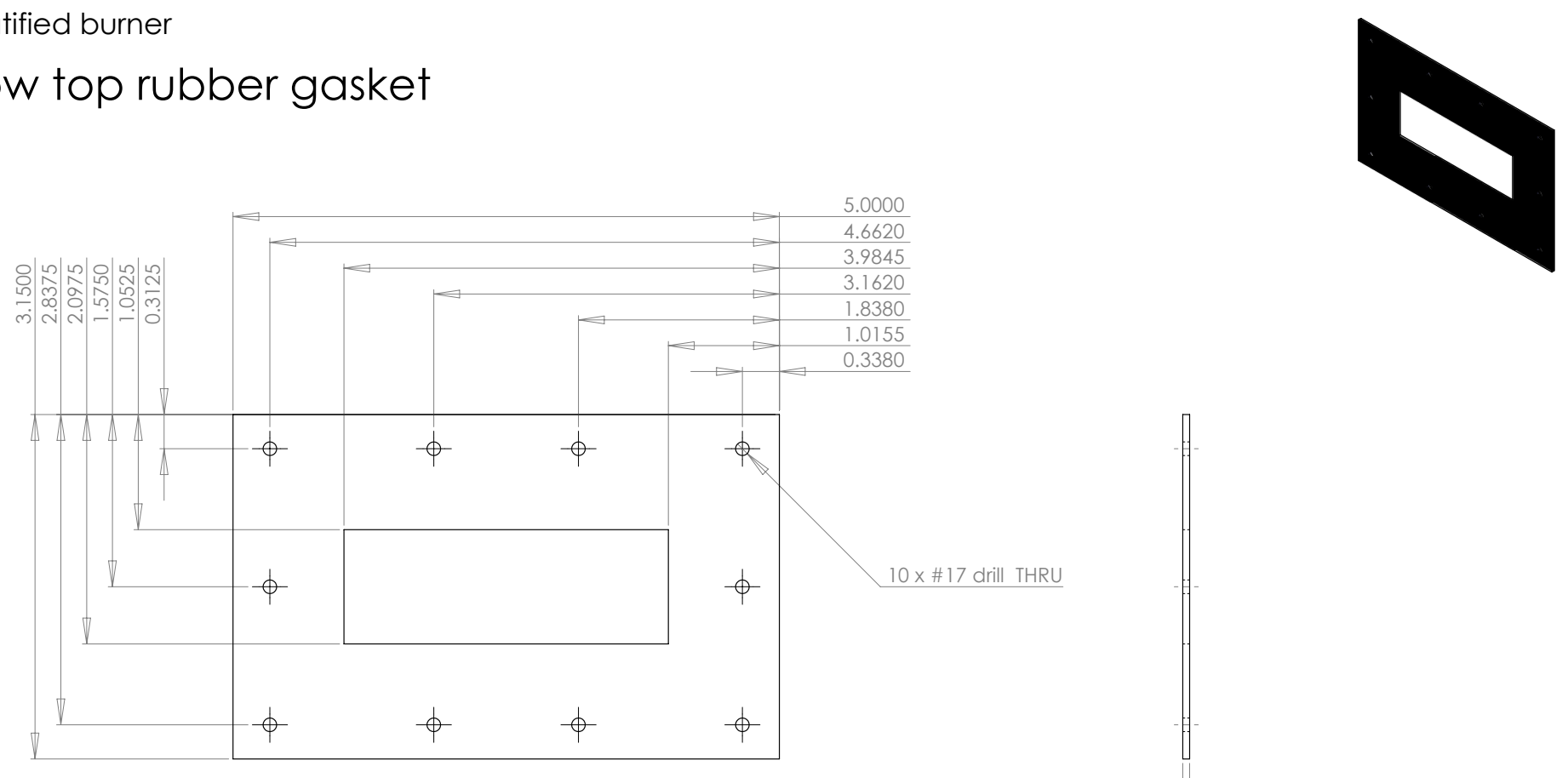

MATERIAL : rubber

ALL DIMENSIONS +/-0.005

$1 \mathrm{OFF}$ 
NRC stratified burner

co-flow top
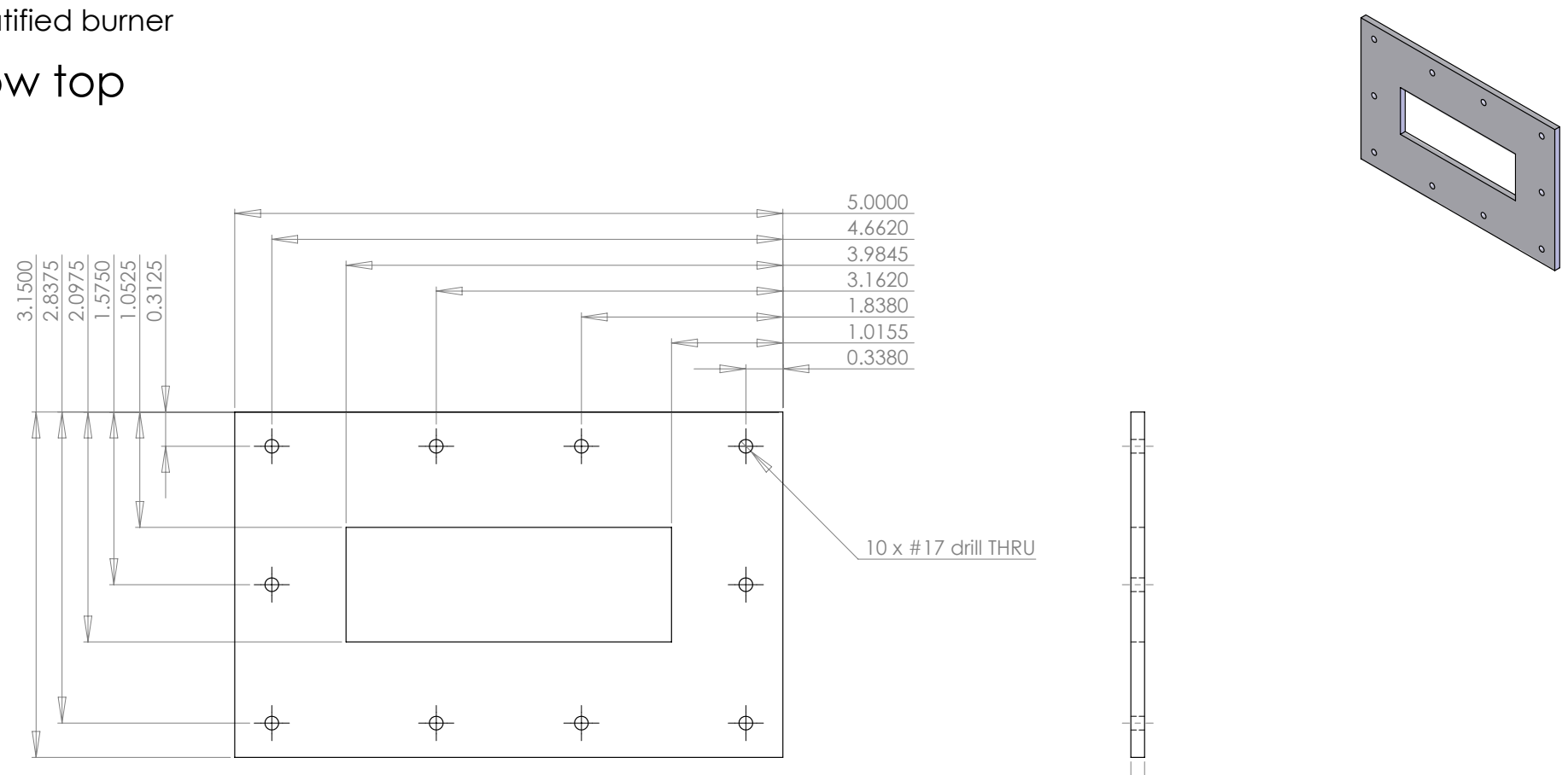

MATERIAL : aluminum

ALL DIMENSIONS +/-0.005

1 OFF 


\section{B.3 Iso-Octane/Air Delivery System}

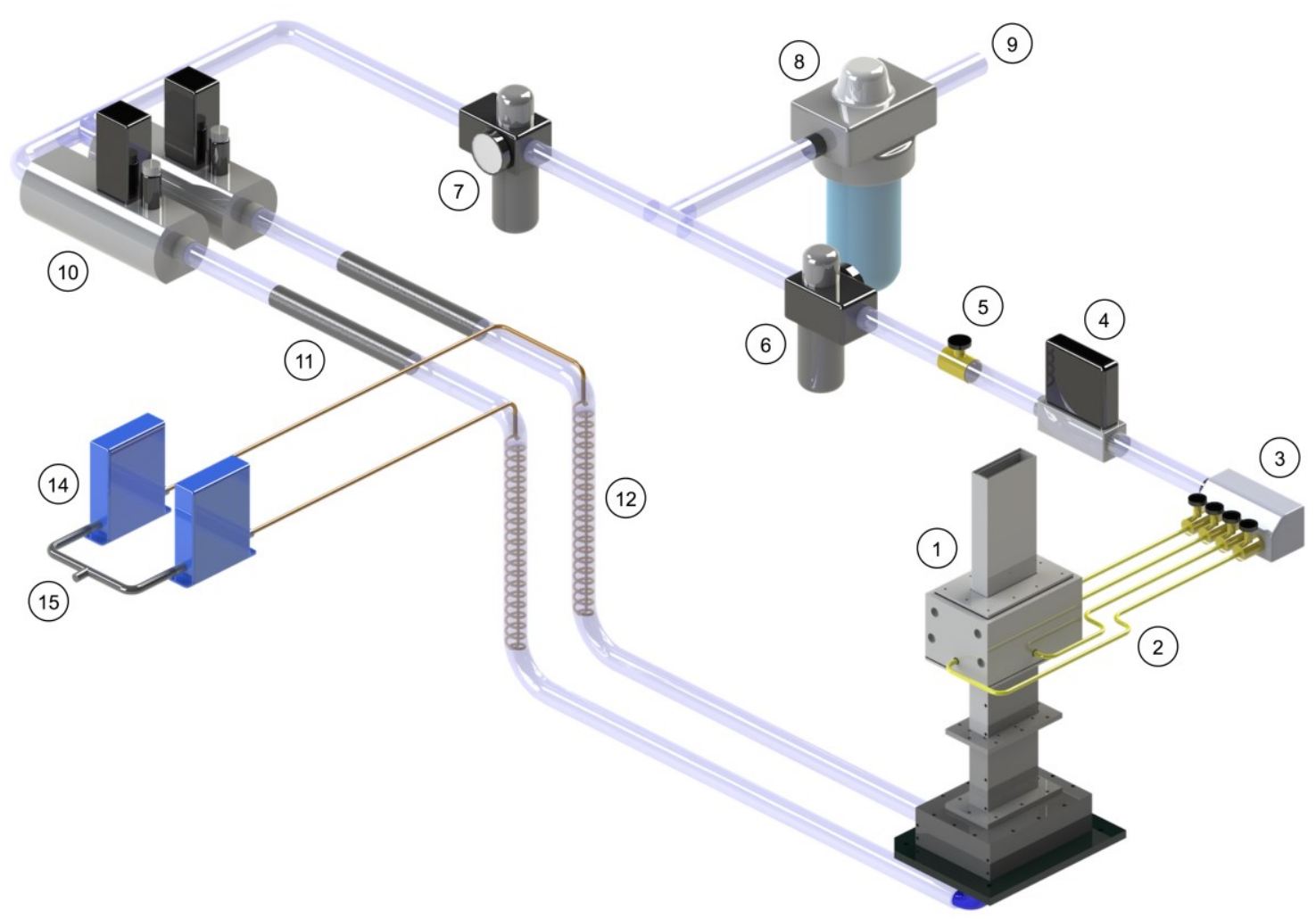

Figure B.6 Schematic of the iso-octane/air delivery system.

(1) NRC stratified slot burner

(2) air co-flow inlet lines

(3) air co-flow distribution manifold

(4) MKS mass flow meter (air)

(5) needle valve

(6) and (7) Schrader Bellows air regulators

(8) Sperry Vickers particulate air filter

(9) compressed air from building lines with nominal pressure of $90 \mathrm{psig}$

(10) Brooks Smart Series gas MFCs (5853S/BE 200 SLPM air full scale)

(11) Omegalux AHP-7561 in-line air heaters

(12) coiled copper iso-octane lines (pre-heated by air)

(13) Horiba LV-F liquid MFCs (LF-F60MO $40 \mathrm{ccm}$ iso-octane full scale, LF-F50MO-094 $25 \mathrm{ccm}$ isooctane full scale)

(14) liquid iso-octane 


\section{Appendix C Further Details of Processing of Individual 3-Pentanone, $\mathrm{OH}$, and $\mathrm{CH}_{2} \mathrm{O}$ PLIF Images}

This appendix provides additional information on the configuration and calibration procedures for PLIF experiments and processing of the acquired instantaneous 3-pentanone, $\mathrm{OH}$, and $\mathrm{CH}_{2} \mathrm{O}$ PLIF images. All images were processed using code written in Matlab, using Matlab's available image processing, curve fitting, and statistics toolboxes.

\section{C.1 Configuration and Calibration Procedures for PLIF Experiments}

There were several considerations in the experimental preparations for subsequent PLIF experiments. These included:

\section{Spatial registration of Intensified Cameras}

As detailed in Sections 2.4 and 3.3.2, to ensure precise spatial correspondence of $\mathrm{OH}$ and $\mathrm{CH}_{2} \mathrm{O}$ PLIF images acquired from each ICCD, a spatial registration procedure was implemented. Two different custom targets, each consisting of a perforated plate with holes arranged on an orthogonal grid, were used to register "input" points on the $\mathrm{CH}_{2} \mathrm{O}$ /biacetyl ICCD to "base" points on the $\mathrm{OH} / 3$-pentanone ICCD. For the data presented in Chapter 2, an initial 90 point target was used. For the data presented in Chapter 3 and Chapter 4, an improved 225 point target with $1 \mathrm{~mm}$ diameter holes more finely spaced on at $5 \mathrm{~mm}$ intervals was used. Images were registered with a "linear conformal" correlation, which was selected following initial tests using a variety of other image registration routines available in the Matlab image processing toolbox including "affine", "projective", "polynomial", "piecewise linear", and "local weighted mean". The "linear conformal" approach was consistently the most accurate as further discussed below. An inverse mapping, "bilinear" interpolation function was used to create a final resampled corrected image, by remapping intensity values from the original image, based on the liner conformal registration. Tests 
comparing with the "bicubic" interpolation function demonstrated that the resulting instantaneous HRR images and ensemble averaged results showed negligible differences. Sample un-registered and registered target images are shown in Figure C.1a and Figure C.1b, which highlight the spatial correspondence of reference "input" and "base" points following registration. Reference points that do not overlap are shown as gray pixels, and reference points that do overlap are shown as white pixels.

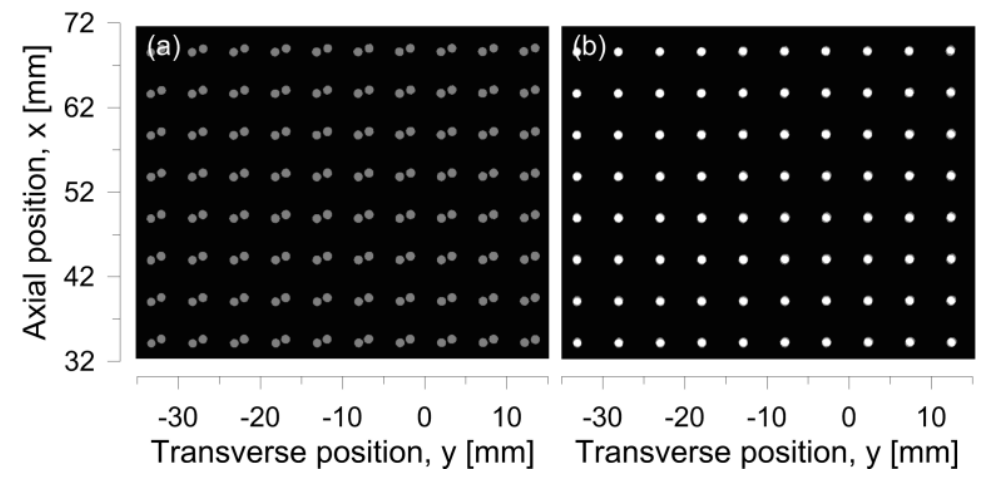

Figure C.1 Superimposed (a) unregistered and (b) registered $\mathrm{CH}_{2} \mathrm{O} /$ biacetyl and $\mathrm{OH} / 3$-pentanone target images.

The accuracy of the spatial registration was tested by measuring the Euclidean distance between the "input" points on the $\mathrm{CH}_{2} \mathrm{O} /$ biacetyl ICCD and the "base" points on the $\mathrm{OH} / 3$-pentanone ICCD once the registration was applied. Results showed excellent agreement, as the Cartesian coordinates of the registered "input" and "base" points were within 0.17 and 0.59 pixels at $95 \%$ confidence, equivalent to 0.011 and $0.040 \mathrm{~mm}$. Camera alignment was verified before each experimental test run, and the cameras were spatially registered prior to running experiments to ensure accurate spatial registration between $\mathrm{OH}$ and $\mathrm{CH}_{2} \mathrm{O}$ images. Lastly, final data were acquired in rapid succession for each of the premixed and gradient cases.

\section{Configuration of fluorescence imaging setup}

Configuration experiments were performed to plan and verify the experimental settings for subsequent laser induced fluorescence measurements. Laser energies were adjusted to below the saturation levels of 3pentanone, $\mathrm{OH}$, and $\mathrm{CH}_{2} \mathrm{O}$ to ensure the fluorescence signals scaled linearly with local laser fluence. For 3pentanone, this was confirmed by imaging the variation in fluorescence as a function of laser energy. A 
quartz cuvette containing iso-octane seeded with trace amounts of 3-pentanone was placed in the path of the laser beam, and the emitted fluorescence was acquired as the laser energy was incrementally adjusted by rotating the half-wave plate positioned at the exit of the Nd:YAG laser. Laser energies corresponding to each increment were measured with an Ophir laser thermal sensor 30 (150) A-HE thermal head equipped with an Ophir NOVA II display. This procedure helped define the linear fluorescence regime for subsequent 3-pentanone PLIF experiments, where the achieved linear relationship between equivalence ratio and 3-pentanone fluorescence is evidenced in the calibration plot shown in Figure 3.15. For $\mathrm{OH}$ and $\mathrm{CH}_{2} \mathrm{O}$, laser fluence estimates were made based on laser energies measured with the same Ophir laser thermal sensor 30 (150) A-HE thermal head and Ophir NOVA II display, and dimensions of the laser sheets that were calculated from the manufacturer quoted laser specifications and the laser sheet forming optics. Laser fluences for both $283 \mathrm{~nm}$ and $355 \mathrm{~nm}$ laser sheets were kept within saturation levels of $\mathrm{OH}$ and $\mathrm{CH}_{2} \mathrm{O}$ respectively.

\section{Pulse to pulse laser intensity corrections}

As explained in Section 3.3, a pair of Thorlabs DET10A Si-based high speed photodiodes was used to monitor and correct for pulse-to-pulse variations in laser energy. The analogue photodiode signal was hardware integrated using a Stanford Research Systems SR280 Boxcar Averager System outfitted with two SR250 Boxcar Gated Integrator Modules. Care was taken to minimize saturation of the photodiodes by selecting a reflection of limited energy density to ensure the integrated signal resembled the expected Gaussian temporal profile of the laser output.

Fluorescence correction functions were determined by plotting the variation in mean fluorescence signal of instantaneous images (normalized by the mean of the entire data set) as a function of the corresponding measured photodiode signal (also normalized by the mean of the entire data set) for the reference premixed case. A linear fit was then applied to the data to obtain the fluorescence correction function, and this was done for 3-pentanone, $\mathrm{OH}$, and $\mathrm{CH}_{2} \mathrm{O}$ during each experimental test run. The resulting correction functions were then used in conjunction with the measured pulse to pulse variations in laser energy to correct the instantaneous PLIF images. Variations in pulse to pulse laser energy were measured to be $2.5 \%$ and $2.2 \%$ at $95 \%$ confidence for the $283 \mathrm{~nm}$ and $355 \mathrm{~nm}$ beams respectively. 
Corrections for Variations in Mean Laser Fluence Profile

As discussed in Sections 2.3 and 3.3, mean laser sheet intensity profiles (i.e. the variation in laser fluence in the axial $\mathrm{x}$ direction) were characterized using tracer PLIF of 3-pentanone (283 nm laser sheet) and biacetyl (355 nm laser sheet) in non-reacting, fully premixed conditions. A profile correction image was generated for each laser sheet by re-scaling these non-reacting tracer PLIF images by dividing all pixel intensity values by the maximum intensity value in the image. Instantaneous 3-pentanone, $\mathrm{OH}$, and $\mathrm{CH}_{2} \mathrm{O}$ PLIF images were normalized with the resulting profile correction images. Variations in shot to shot laser profile were quantified, and measured to be $6.3 \%$ and $8.2 \%$ at $95 \%$ confidence for the $283 \mathrm{~nm}$ and $355 \mathrm{~nm}$ beams respectively.

\section{C.2 Image Processing Flow Charts and Sample Intermediate Images for 3-Pentanone, $\mathrm{OH}$, and $\mathrm{CH}_{2} \mathrm{O}$ PLIF}

This section provides flow charts outlining the processing routines applied to the instantaneous 3pentanone, $\mathrm{OH}$, and $\mathrm{CH}_{2} \mathrm{O}$ PLIF images. Sample images corresponding to each step are also included.

Figure C.2 details each operation in the processing of the instantaneous 3-pentanone PLIF images, starting from the raw fluorescence images acquired from the ICCD, through to the generation of equivalence ratio maps of the reactants. The resulting $\phi$ images were used to characterize the reactant flow field, and to determine the position and width of the analysis region of interest (ROI) specific to each gradient flame setting following procedures detailed in Sections 2.3.2, 3.3.1, and 4.3. Equivalence ratio data were also used in combination with simulated HRR data for premixed flames in Monte Carlo simulations to assess the combined effects of turbulent mixing (by accounting for the broader range of $\phi$ within the ROI among gradient conditions) and temperature induced bias errors on the $\mathrm{OH}$ and $\mathrm{CH}_{2} \mathrm{O}$

fluorescence signals (by accounting for Boltzmann population fractions and collisional quenching rates of both species) on the mean HRR within the ROI. Further details on this approach are provided in Sections 3.3.1, 3.3.2, and 4.3. 


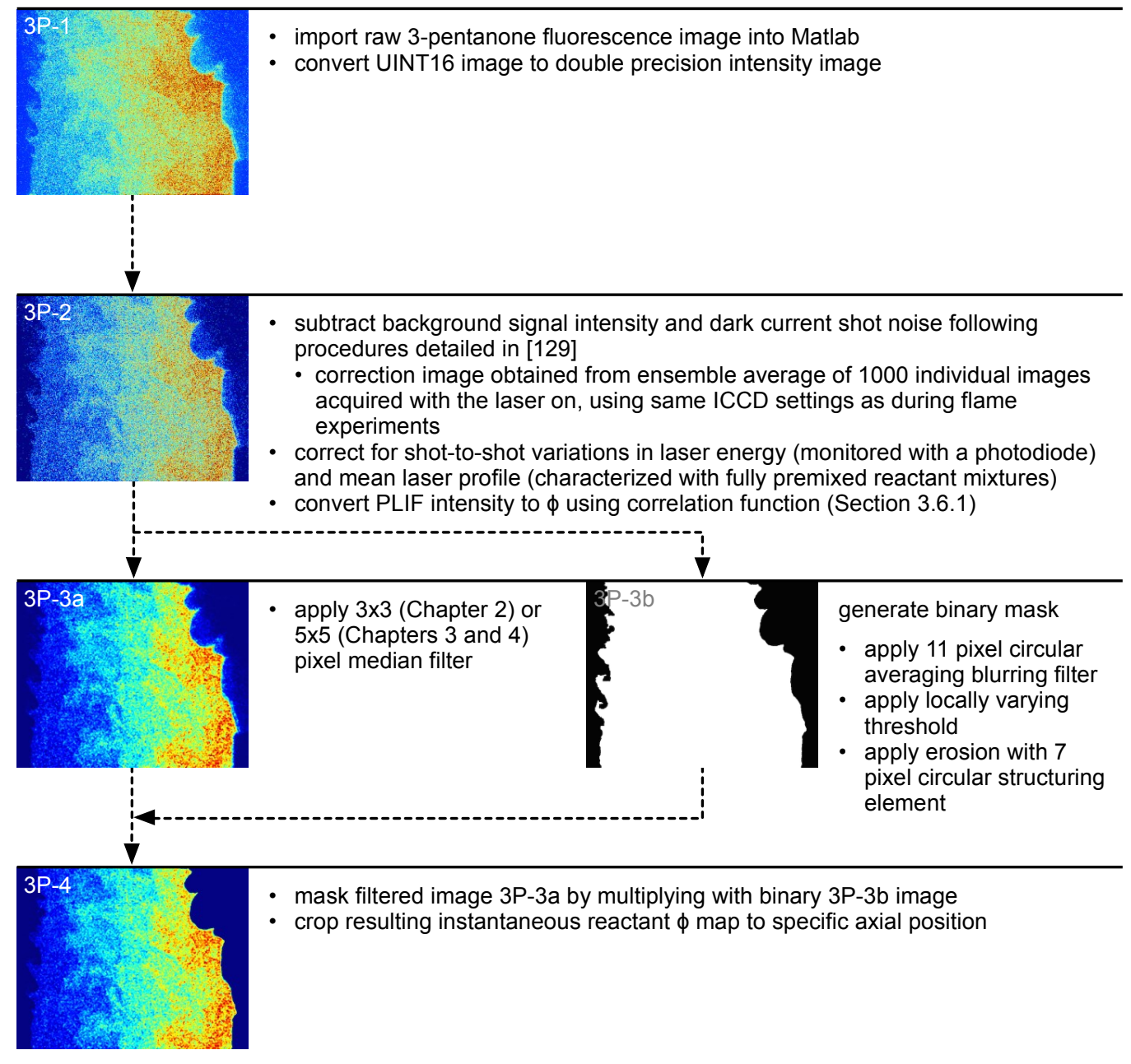

Figure C.2 Flow chart showing sample images corresponding to each operation in the processing of individual 3-pentanone PLIF images.

Instantaneous $\mathrm{OH}$ and $\mathrm{CH}_{2} \mathrm{O}$ PLIF images were processed following the operations outlined in Figure C.3 and Figure C.4 respectively. They were subsequently used to evaluate the effect of mean $\phi$ gradient on flame front topology, and more specifically to calculate the mean reaction progress variable $\langle\mathrm{c}\rangle$ (refer to Sections 2.4.1 and 3.3.3 for details on the data reduction procedures), the turbulent flame brush thickness $\mathrm{L}_{\mathrm{t}}$ (Section 3.3.3), the flame surface density $\Sigma$ (Section 2.4.2), curvature $\kappa$ (Sections 2.4 .3 and 4.4.1), and mean flame length (Section 3.4.3). The product of spatially registered $\mathrm{OH}$ and $\mathrm{CH}_{2} \mathrm{O}$ fluorescence intensity data was used to obtain information on the heat release rate (HRR) based on the approach of Paul and Najm [55], which is described in Section 3.3.2. The resulting HRR images were studied to assess the influence of mean $\phi$ gradient on the ensemble average of HRR (Sections 3.4.2 and 
3.4.3), ensemble average HRR vs $\langle\mathrm{c}\rangle$ (Section 3.4.2), and ensemble average of HRR / flame length and total HRR (Section 3.4.3). Individual HRR contours were further examined for variations in the ensemble average of the instantaneous peak HRR and FWHM thickness $\delta_{\mathrm{t}}$ (Section 4.4.3), as well as their distribution as a function of local curvature (Sections 3.4.2 and 4.5).

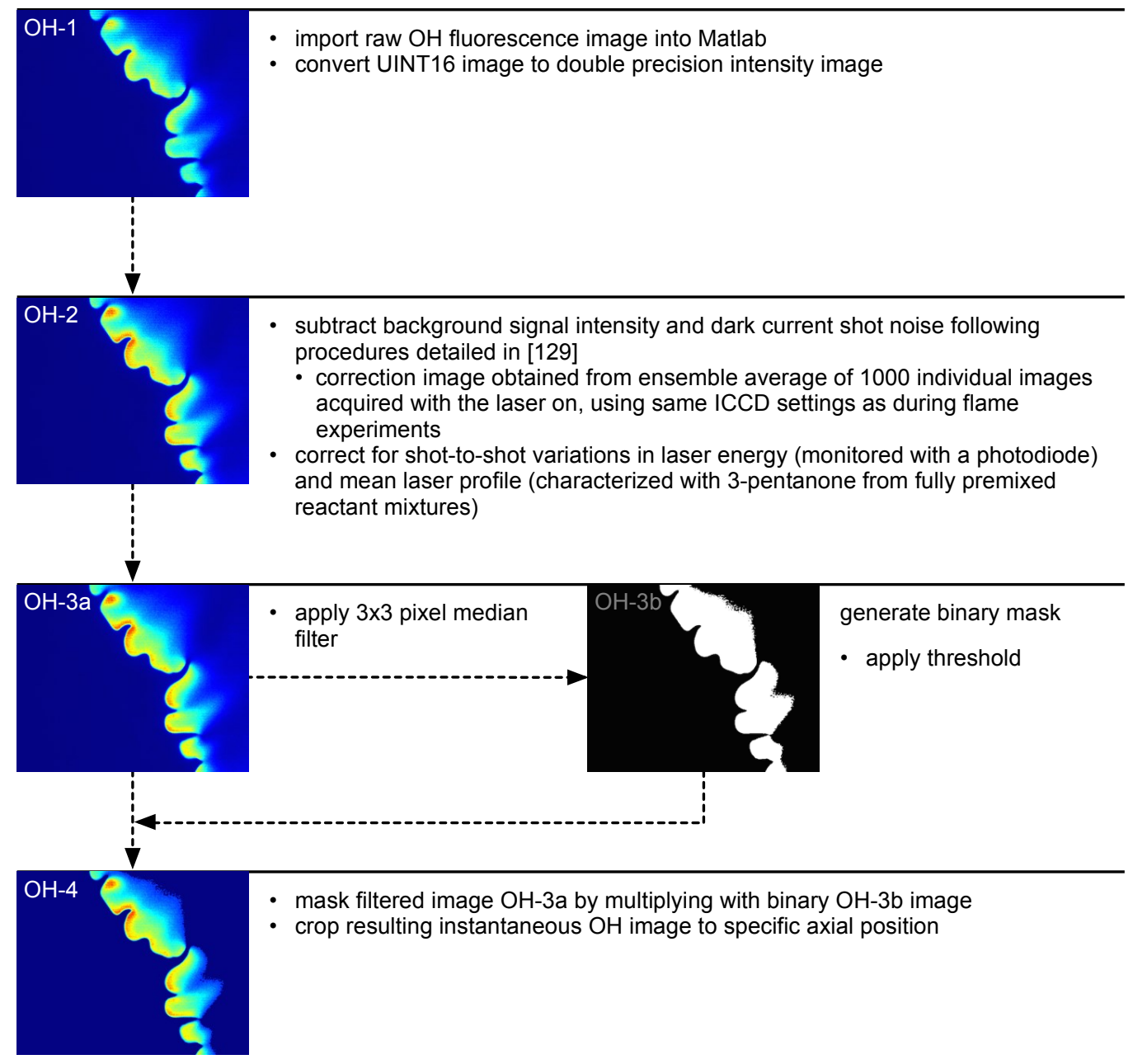

Figure C.3 Flow chart showing sample images corresponding to each operation in the processing of individual OH PLIF images. 


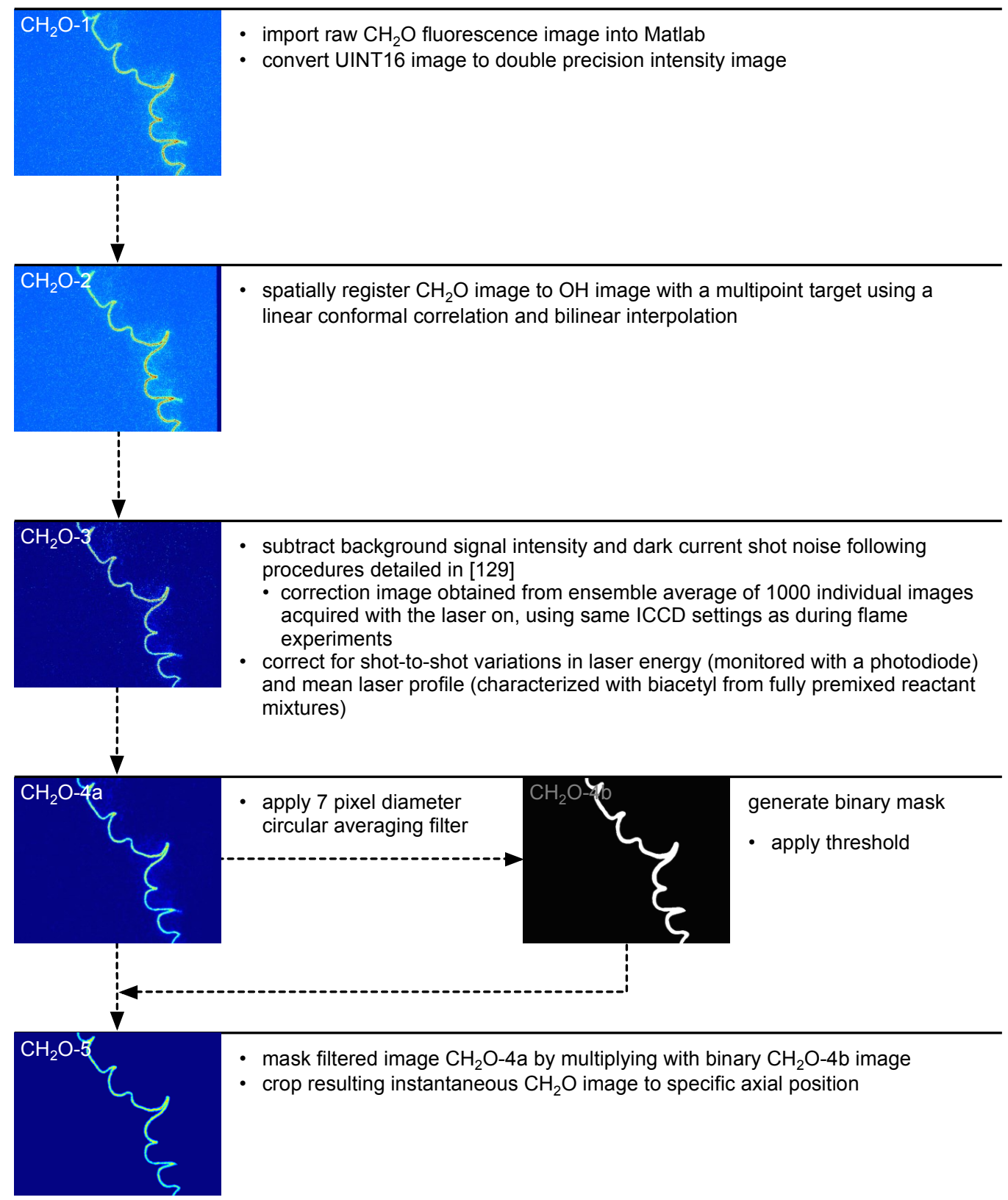

Figure C.4 Flow chart showing sample images corresponding to each operation in the processing of individual $\mathrm{CH}_{2} \mathrm{O}$ PLIF images. 